\title{
Magnetic Survey of the Hydroflouric Acid Spill Area
}

by

\author{
R. J. Cumbest
}

Westinghouse Savannah River Company

Savannah River Site

Aiken, South Carolina 29808

DOE Contract No. DE-AC09-89SR18035

This paper was prepared in connection with work done under the above contract number with the U.S. Department of Energy. By acceptance of this paper, the publisher and/or recipient acknowledges the U.S. Government's right to retain a nonexclusive, royalty-free license in and to any copyright covering this paper, along with the right to reproduce and to authorize others to reproduce all or part of the copyrighted paper. 


\section{DISCLAIMER}

This report was prepared as an account of work sponsored by an agency of the United States Government. Neither the United States Government nor any agency thereof, nor any of their employees, makes any warranty, express or implied, or assumes any legal liability or responsibility for the accuracy, completeness, or usefulness of any information, apparatus, product, or process disclosed, or represents that its use would not infringe privately owned rights. Reference herein to any specific commercial product, process, or service by trade name, trademark, manufacturer, or otherwise does not necessarily constitute or imply its endorsement, recommendation, or favoring by the United States Government or any agency thereof. The views and opinions of authors expressed herein do not necessarily state or reflect those of the United States - Government or any agency thereof.

This report has been reproduced directly from the best available copy.

Available to DOE and DOE contractors from the Office of Scientific and Technical Information. P. O. Box 62, Oak Ridge. TN 37831; prices available from (615) $576-8401$.

Available to the public from the National Technical Information Service. U. S. Department of Commerce, 5285 Por Royal Rd., Springfield, VA 22161 


\section{DISCLAIMER}

Portions of this document may be illegible in electronic image products. Images are produced from the best available original document. 


\section{Magnetic Survey of the Hydrofluoric Acid Spill Area}

\section{EXECUTIVE SUMMARY}

A magnetic survey was conducted at the Hydroflouric Acid Spill Area to evaluate the possibility of buried metal containers or other ferric objects. A proton precession gradiometer with the bottom sensor $2 \mathrm{ft}$ and the top sensor $4 \mathrm{ft}$ from the ground surface was used to sample the survey area on a grid defined by 1 meter grid nodes in a square array.

The survey area was surrounded on three sides by a metal fence and metal recycling facility. The fence and metal objects adjacent to the fence, in addition to metal associated with hydrologic monitoring well heads, waste unit marker balls and metal signs caused significant magnetic field and gradient anomalies that extended throughout most of the survey area. In addition to these easily explained anomalies, several anomalies were detected which probably result from buried ferric objects. Some of these anomalies are of limited aerial extent and magnitude and probably represent small shallow objects.

However, at least one of the anomalies is of sufficient magnitude and extent that it may represent a large metal container such as a metal drum.

\section{INTRODUCTION AND SCOPE}

The Hydrofluoric Acid Spill Area is located in the southwest portion of the Central Shops facility on Savannah River Site (Fig. 1). The disposal mode and history of this site are not well known, and it is uncertain if acid was only spilled, or if acid contaminated soil or acid filled containers were buried at the site.. In order to evaluate the presence of buried metallic containers a magnetic survey was conducted at the Hydrofluoric Acid Spill Area by the Site Geotechnical Services Department during September, 1994, at the 
request of the Environmental Restoration Department. The magnetic survey consists of a grid over the site defined by a 1 meter square grid node pattern.

\section{Magnetic Survey Concepts}

The magnitude of the magnetic field measured near the ëarth's surface is influenced by several factors. The main contribution to the total intensity is due to the internal magnetic field of the earth produced in the vicinity of the earth's core. However, this field is modified by the solar wind of charged particles produced by electromagnetic activity on the sun. This solar wind distorts the magnetic field produced by the earth into a tear drop shape with the blunt end pointing toward the sun. More importantly, the effect of the solar wind is to produce time variations in the total field. The earth's rotation, in effect, introduces a diurnal variation with a twenty four hour period. Also, superimposed on this well defined twenty four hour cycle are variations with no well defined period produced by the chaotic nature of the suns electromagnetic activity. Typically these variations manifest themselves as micropulsations on the order of less than $10 \mathrm{nT}$, but significantly larger variations can be produced by magnetic storms on the sun (i.e. sunspots). The net result of these variations is illustrated in figure 2 .

The earth's magnetic field is also modified by features near the surface that produce localized spatial variations, or anomalies, in the field intensity. The use of magnetic techniques in searching for buried metallic objects is a consequence of the ability of iron and iron alloys to cause relatively large amplitude anomalies. These anomalies are produced by two effects. Some materials have properties that allow them to become magnetized in the presence on an external magnetic field. This process is called induction. The external magnetic field is the inducing field and the field produced by the material the induced field. The total field $(\overrightarrow{\mathbf{B}})$ now becomes the sum of the inducing external field $(\overrightarrow{\mathbf{H}})$ and the field produced by the material $\left(\frac{\overrightarrow{\mathbf{H}^{\prime}}}{}\right)$. This relationship can be expressed: 
Hydroflouric Acid Spill Magnetic Survey

$$
\overrightarrow{\mathbf{B}}=\overrightarrow{\mathbf{R}^{2}}+\overrightarrow{\mathbf{F}^{\prime}}
$$

In addition some materials possess a magnetic field independent from any external source. This field is typically acquired at some time in the past history of the material and is called remnant magnetization. For most magnetic survey applications the magnitude of the induced field is assumed to be much greater than the remnant magnetization and this assumption has been shown to be valid in the specific case of standard 55 gallon metal drums (Gilkeson and others, 1992).

In reality magnetic fields always manifest as dipoles. That is north and south magnetic poles occur in pairs to produce dipole fields. A field line representation of a dipole and monopole field are illustrated in figure 3. In this type of representation the density of the field lines is proportional to the field magnitude. To a first approximation, the magnetic field produced near the core of the earth can be considered a single dipole with the magnetic field lines impinging the surface of the earth at 90 degrees at the north and south magnetic poles and parallel to the surface of the earth at the magnetic equators.

The geometric relationship of the magnetic field lines to the geographic poles is expressed by magnetic inclination and declination. Declination describes the angular relationship of the component of the field line parallel to the surface of the earth, to geographic north. The magnetic inclination expresses the angular relationship between the magnetic field lines and the horizontal (Fig. 4). Although, these angular relationships change slowly due to poorly understood long term variations, the declination in the vicinity of SRS is about 4 degrees to the west and the inclination about 65 degrees.

Although inducing bodies usually possess highly irregular shapes and sizes they can be usefully modeled as dipoles or arrays of dipoles. In some instances, if the area of consideration is near one end of a dipole relative to the dipole separation, the local field can be considered to be produced by a single pole and approximates monopole behavior. The anomalous fields produced by a dipole and monopole for a magnetic inclination of 60 
Hydroflouric Acid Spill Magnetic Survey

degrees in the northern hemisphere (i.e. conditions very similar to SRS) and their resultant effect on the total magnetic field measured near the body are illustrated in figure 3 . Notice that the anomalous field lines on the north side of the dipole are opposite in sense to the ambient field therefore reducing the field strength in this location. In contrast the anomalous field lines on the south side are in the same sense resulting in an increased field strength. The monopole exhibits similar behavior although not as extreme. This phenomena is the explanation for the asymmetrical shaped magnetic highs and lows produced by inducing bodies at mid-latitudes on the earth. Figure 5 illustrates typical magnetic profiles that would be observed at the latitude of SRS over monopoles and dipoles in addition to a generalized contour representation of a dipole, with illustrative profiles across the contours.

Generalized magnetic field intensity profiles across more complicated shapes for an inclination of 60 degrees are illustrated in figure 6 . These profiles all exhibit the typical asymmetrical signatures along the north - south profiles with magnetic highs occurring over southern portions of the bodies.

The previous discussion has been presented in the context of the intensity of the total magnetic field. However, another measurement which can be very useful in interpreting magnetic anomalies is the vertical magnetic gradient, which changes much more rapidly with distance than the total field. This effect results.in edge enhancement of anomalies and more effective anomaly separation (Fig. 7). The vertical magnetic gradient can be determined by making measurements of the total magnetic intensity at two known elevations. The vertical gradient is then determined by the difference between the two measurements divided by their separation. The two measurements can be made by a single sensor instrument by moving the sensor between the two measurements or by an instrument with two separated sensors.

In order to conduct a magnetic survey magnetic data are collected at discrete intervals either along a traverse or over a 2 dimensional grid. In practice, data for 2-D 
grids are usually collected as multiple traverses. Traverse data can be conveniently displayed as magnetic intensity profiles. 2-D gridded data are usually contoured and displayed as contour diagrams. Displayed in this fashion these data can be qualitatively interpreted by comparing them with profiles or contours that would be predicted from bodies of known shape and size as presented earlier.

In addition to qualitative information, profiles and contour diagrams may also be used for quantitative analysis if the magnetic response of specific objects is known. Due to their widespread use in the past as containers for industrial materials 55 gallon steel drums are commonly targeted during characterization of waste sites. Gilkeson and others (1992) have evaluated the magnetic response from single and multiple drums buried at different depths. The magnetic response is provided by a model calibrated from actual measurements on a 55 gallon drum. These anomalies were calculated for a total field intensity of $53220 \mathrm{nT}$ and inclination of 70 degrees. Conditions very similar to SRS.

Figure 8 illustrates model total field and vertical gradient profiles over a single drum, in vertical position, with the drum top at different depths below the sensor. If single, deeply buried drums are the target, these profiles make clear the need to locate the sensor near the ground surface in order to minimize the distance between the sensor and drum. For sensor - drum distances greater than about $20 \mathrm{ft}$ the anomaly amplitude has decreased to values that may not be discernible from noise.

Figure 9 illustrates the contour representation of an anomaly for a single drum with its top located $10 \mathrm{ft}$ below the sensor. Also shown in figure 9 are the station locations of a typical $5 \mathrm{ft}$ by $5 \mathrm{ft}$ grid over the anomaly. Note that a misfortunate placement of a $20 \mathrm{ft}$ by $20 \mathrm{ft}$ grid may fail to detect the anomaly at all and that a badly placed $10 \mathrm{ft}$ by $10 \mathrm{ft}$ grid would only reveal a maximum amplitude on the order of $25 \mathrm{nT}$ which may also not be discernible from noise and at best would offer poor resolution concerning the drum location. 
Hydroflouric Acid Spill Magnetic Survey

Figure 10 illustrates the contour representation of a total field magnetic anomaly for a cache of 5 drums located in a $12 \mathrm{ft}$ by $12 \mathrm{ft}$ area with the drum tops $10 \mathrm{ft}$ below the sensor. Note again the a $20 \mathrm{ft}$ by $20 \mathrm{ft}$ grid may fail to detect the anomaly. A $10 \mathrm{ft}$ by $10 \mathrm{ft}$ grid would crudely resolve the anomaly. However, Gilkeson and other's (1992) modeling demonstrated that at a depth of $10 \mathrm{ft}$ if the center drum were removed the drums on the corners would be resolved into individual anomalies. If this type of information is required from the magnetic survey then $5 \mathrm{ft}$ grid node separations would be needed to fully resolve the individual anomalies associated with each drum.

In addition to the above considerations other factors enter into the design of an effective magnetic survey. Although smaller grid spacings allow better resolution of anomalies they are also labor intensive. Placing the sensor near the ground makes it much more sensitive to small, shallow sources such as metallic trash or magnetite variations in the soil. This is especially a problem for gradiometer surveys because these effects are amplified. In addition gradiometers are most effective when the sensor separation is $1 / 5$ or less the distance to the target. If the sensors are placed near the ground this condition may not be met for shallow targets. A careful consideration of what information is needed from the magnetic survey and the resources available will determine which of the aspects discussed above are most important.

\section{Description of Survey Site}

A planimetric map of the Hydrofluoric Acid Spill Area is presented in Figure 11. The survey area occurs in a recess in a chain-link fence that surrounds a metal recycling facility in the Central Shops Area. Several large metal objects, including dumpsters, metal tanks, a pile of aluminum cans, and various other types of metal debris and office furniture, were located adjacent to the fence in the metal recycling facility at the time of the survey. The facility uses an electromagnetic crane to move the scrap metal so that it was necessary to perform the survey at times when the crane was not operating. 
Southwest of the survey area several abandoned metal storage tanks were located approximately $100 \mathrm{ft}$ away.

The most prominent objects in the survey area are 4 hydrologic monitoring well heads (CSA1-4) with their associated metal signs and posts. Also, four orange HP waste unit marker balls occur in a square pattern in the center of the survey area. This area is also marked by a large metal sign. In addition to these prominent objects a metal object can be seen on the surface at coordinates $(50172,61690)$.

\section{MAGNETIC SURVEY}

\section{Survey and Analytical Techniques}

The grid established for the magnetic survey of the Hydroflouric Acid Spill Area (fig. 12) was defined by a 1 meter grid node spacing in a square array. The grid was established to give coverage to the majority of the area enclosed by the recess in the fence and surrounding the orange waste unit marker balls. The survey lines were oriented perpendicular to the northern fence for convenience. The grid station locations were established with a transit to mark 90 degree angles and a cord marked at 1 meter intervals to determine distances.

Magnetic data were collected with an EG\&G Geometrics G-856 Gradiometer with the top sensor at 1.22 meters ( $4 \mathrm{ft}$ ) from ground level and the bottom sensor 0.61 meters ( $2 \mathrm{ft}$ ) from ground level. This configuration places the sensors near the ground to maximize the sensitivity to small near surface or deeply buried objects. This instrument has a nominal accuracy of $0.1 \mathrm{nT}$. However, high magnetic gradients ( $>600 \mathrm{nT} / \mathrm{m}$ ) will cause the signal to be sharply degraded. In most cases this will cause the accuracy to deteriorate to $1 \mathrm{nT}$. More severe degradation can result in significant accuracy losses resulting in poor data very near large metallic objects. As a QA/QC check and in order to account for 
diurnal variation a single station (grid node 0,25 ) was occupied several times during the survey.

The data were downloaded from the magnetometer through a RS-232 port to an IBM PC Model 80. After downloading, the data were assigned coordinates and diurnally corrected with MAGLOC, software provided by EG\&G. Further data display and processing, including gridding and contouring were accomplished with software provided by GEOSOFT Inc. Gridding of the data was accomplished with RANGRID, which fits a minimal curvature surface to the gridded data. Magnetic gradients were calculated by subtracting the top sensor reading from the bottom sensor and dividing by their separation. Since the top and bottom sensor readings are taken at a relatively short time scale (approximately 3 seconds) relative to diurnal variations the gradient is calculated from the data before diurnal correction. The data for the survey along with daily diurnal variations are tabulated in the Appendix.

\section{Magnetic Data Set}

Several anomalies are present in the magnetic data (Figs. 13-15). The most prominent magnetic anomaly occurs parallel to the metal fence that surrounds the survey area on three sides. This anomaly exhibits a positive gradient away from the fence lines and can bee seen to influence almost the entire survey area. Although the effects of the fence anomaly can be seen throughout the survey area other prominent isolated anomalies occur in the locations of the hydrologic monitoring well heads and the HP waste unit marker balls and sign. The influence of these anomalies are broad compared to the separation of these features so that in many places the anomalies coalesce in the total field data for the top and bottom sensors. However, as expected these anomalies are better resolved and separated by their vertical gradients. 
Hydroflouric Acid Spill Magnetic Survey

Other anomalies not associated with prominent features on the waste unit occur approximately centered on coordinates: $(50170,61690) ;(50205,61740),(50205,61760)$ and $(51220,61775)$ as illustrated in figure 16.

\section{DISCUSSION}

Because of its extent, the large magnetic anomaly associated with the fence causes interference with other anomalies throughout the survey area. This can be attributed to the metallic composition of the fence and the presence of several large ferric metal objects located immediately adjacent to the fence in the Central Shops Metal Recycling Facility. The more isolated anomalies in the locations of the hydrologic monitoring well heads and HP waste unit marker balls and sign can be attributed to the ferric compositions of the metallic parts of these features. These anomalies also cause interference throughout the survey area. In addition to these anomalies, the isolated dipole anomaly located at coordinates $(50170,61690)$ occurs in the location of a metal object that can be seen on the surface (Figure 16). The presence of the anomalies caused by these metal objects on the surface complicate the detection potential of anomalies caused by ferric objects.

The isolated dipole anomalies that cannot be explained by features seen on the surface probably represent buried ferric metal objects. The relatively small anomalies at $(51220,61775)$, and $(50205,61740)$, are of extremely localizẹd extent and must represent relatively small, callously buried objects. The other anomaly $(50205,61665)$ probably represents a ferric object of significant depth and extent.

\section{CONCLUSIONS}

Several anomalies are present in the magnetic field and gradient data from the Hydroflouric Acid Spill Area. The largest of these anomalies can be attributed to metallic features on the surface of the survey area or immediately adjacent to the survey area. Most 
Hydroflouric Acid Spill Magnetic Survey

of the smaller anomalies identified in the area probably result from relatively small shallow, ferric objects that are not large enough to represent a metallic container of any significant volume. However, at least one anomaly was identified that could represent a buried metallic container such as a drum.

\section{REFERENCES}

Breiner, S. Applications Manual for Portable Magnetometers, Sunneyvale, California 94084 (1973)

Gilkeson, R. H., Gorin, S. R., Laymon, D.E. (1992) Application of magnetic and electromagnetic methods to metal detection. Proceedings of the Symposium on the Application of Geophysics to Engineering and Environmental Problems, v.1, p.309328.

\section{..}

Telford, W. M., Geldart, L. P., Sheriff, R. E., Keys, D. A., (1978) Applied Geophysics. Cambridge University Press, 860p: 


\section{APPENDIX: Hydroflouric Acid Spill Magnetic Survey Data}

\begin{tabular}{|c|c|c|c|c|c|}
\hline & \multicolumn{5}{|c|}{ DAT } \\
\hline & TOP & & & BOTTOM & \\
\hline Sta. & Time & Field $(n T)$ & Sta. & Time & Field (nT) \\
\hline 1 & $11: 28: 30$ & 51395.2 & 0 & $11: 28: 27$ & 51408.4 \\
\hline 3 & $11: 28: 39$ & 51394.8 & 2 & $11: 28: 35$ & 51408.0 \\
\hline 5 & $11: 28: 47$ & 51394.8 & 4 & $11: 28: 44$ & 51407.8 \\
\hline 7 & $11: 28: 55$ & 51394.8 & 6 & $11: 28: 52^{m}$ & 51406.8 \\
\hline 9 & $11: 29: 03$ & 51394.4 & 8 & $11: 29: 00$ & 51406.8 \\
\hline 221 & $11: 54: 03$ & 51390.8 & 220 & $11: 53: 59$ & 51402.0 \\
\hline 223 & $11: 54: 11$ & 51391.4 & 222 & $11: 54: 08$ & 51402.6 \\
\hline 225 & $11: 54: 19$ & 51391.6 & 224 & $11: 54: 16$ & 51401.8 \\
\hline 227 & $11: 54: 28$ & 51391.8 & 226 & $11: 54: 24$ & 51401.4 \\
\hline 229 & $11: 54: 36$ & 51392.0 & 228 & $11: 54: 33$ & 51402.0 \\
\hline 443 & $12: 20: 54$ & 51396.2 & 442 & $12: 20: 50$ & 51409.3 \\
\hline 445 & $12: 21: 02$ & 51396.7 & 444 & $12: 20: 59$ & 51410.3 \\
\hline 447 & $12: 21: 10$ & 51397.2 & 446 & $12: 21: 07$ & 51410.6 \\
\hline 449 & $12: 21: 18$ & 51397.0 & 448 & $12: 21: 15$ & 51410.5 \\
\hline 451 & $12: 21: 26$ & 51396.6 & 450 & $12: 21: 23$ & 51411.0 \\
\hline 559 & $12: 35: 03$ & 51398.1 & 558 & $12: 34: 59$ & 51416.0 \\
\hline 561 & $12: 35: 11$ & 51398.2 & 560 & $12: 35: 07$ & 51415.5 \\
\hline 563 & $12: 35: 19$ & 51398.2 & 562 & $12: 35: 15$ & 51415.2 \\
\hline 565 & $12: 35: 27$ & 51398.1 & 564 & $12: 35: 23$ & 51415.5 \\
\hline 567 & $12: 35: 35$ & 51398.1 & 566 & $12: 35: 32$ & 51415.2 \\
\hline 672 & $12: 53: 30$ & 51400.5 & 673 & $12: 53: 33$ & 51413.4 \\
\hline 674 & $12: 53: 38$ & 51401.7 & 675 & $12: 53: 41$ & 51413.8 \\
\hline 676 & $12: 53: 46$ & 51401.6 & 677 & $12: 53: 49$ & 51413.6 \\
\hline 678 & $12: 53: 55$ & 51401.7 & 679 & $12: 53: 58$ & 51413.5 \\
\hline 680 & $12: 54: 03$ & 51401.9 & 681 & $12: 54: 06$ & 51412.1 \\
\hline 781 & $13: 04: 55$ & 51404.0 & 780 & $13: 04: 51$ & 51416.2 \\
\hline 783 & $13: 05: 02$ & 51404.8 & 782 & $13: 04: 58$ & 51416.1 \\
\hline 784 & $13: 05: 05$ & 51403.3 & 785 & $13: 05: 14$ & 51414.7 \\
\hline 786 & $13: 05: 18$ & 51403.1 & 787 & $13: 05: 22$ & 51414.9 \\
\hline 788 & 13:05:26 & 51402.9 & 892 & $13: 17: 14$ & 51414.1 \\
\hline 891 & $13: 17: 11$ & 51406.2 & 894 & 13:1广:22 & 51413.8 \\
\hline 893 & $13: 17: 19$ & 51405.8 & 896 & $13: 17: 30$ & 51413.7 \\
\hline 895 & $13: 17: 27$ & 51405.7 & 898 & $13: 17: 39$ & 51413.8 \\
\hline 897 & $13: 17: 35$ & 51405.9 & 900 & $13: 17: 47$ & 51413.6 \\
\hline 899 & $13: 17: 43$ & 51406.0 & 1001 & $13: 29: 34$ & 51419.0 \\
\hline 1002 & $13: 29: 37$ & 51406.0 & 1003 & $13: 29: 46$ & 51418.3 \\
\hline 1004 & $13: 29: 49$ & 51407.1 & 1005 & $13: 29: 55$ & 51417.7 \\
\hline 1006 & $13: 29: 58$ & 51406.7 & 1007 & 13:30:04 & 51417.4 \\
\hline 1008 & $13: 30: 07$ & 51406.3 & 1009 & $13: 30: 13$ & 51418.1 \\
\hline 1010 & $13: 30: 16$ & 51406.1 & 1107 & $13: 42: 13$ & 51416.2 \\
\hline 1108 & $13: 42: 16$ & 51404.5 & 1109 & $13: 42: 20$ & 51416.1 \\
\hline 1110 & $13: 42: 23$ & 51404.4 & 1111 & $13: 42: 27$ & 51416.0 \\
\hline 1112 & $13: 42: 30$ & 51404.3 & 1113 & $13: 42: 36$ & 51415.9 \\
\hline 1114 & $13: 42: 39$ & 51404.4 & 1115 & $13: 42: 44$ & 51416.1 \\
\hline 1116 & $13: 42: 47$ & 51404.7 & 1211 & $13: 54: 41$ & 51421.7 \\
\hline 1212 & $13: 54: 44$ & 51406.2 & 1213 & $13: 54: 48$ & 51421.4 \\
\hline
\end{tabular}


Hydroflouric Acid Spill Magnetic Survey Data

$\begin{array}{llllll}1214 & 13: 54: 52 & 51406.0 & 1215 & 13: 54: 56 & 51421.3 \\ 1216 & 13: 54: 59 & 51406.1 & 1217 & 13: 55: 02 & 51421.5 \\ 1220 & 13: 55: 14 & 51405.9 & 1219 & 13: 55: 10 & 51421.7 \\ 1314 & 14: 07: 06 & 51405.9 & 1313 & 14: 07: 03 & 51424.6 \\ 1315 & 14: 07: 10 & 51407.9 & 1316 & 14: 07: 17 & 51423.3 \\ 1317 & 14: 07: 20 & 51407.4 & 1318 & 14: 07: 26 & 51422.7 \\ 1319 & 14: 07: 29 & 51409.0 & 1320 & 14: 07: 36 & 51422.9 \\ 1321 & 14: 07: 39 & 51408.7 & 1410 & 14: 24: 00 & 51417.8 \\ 1411 & 14: 24: 03 & 51407.0 & 1412 & 14: 24: 09 \cdots & 51417.6 \\ 1413 & 14: 24: 12 & 51407.2 & 1414 & 14: 24: 17 & 51417.3 \\ 1415 & 14: 24: 20 & 51406.9 & 1416 & 14: 24: 26 & 51417.5 \\ 1417 & 14: 24: 30 & 51407.1 & 1418 & 14: 24: 35 & 51418.2 \\ 1419 & 14: 24: 38 & 51407.1 & 1420 & 14: 34: 26 & 51412.6 \\ 1421 & 14: 34: 30 & 51410.6 & 1422 & 14: 34: 35 & 51412.7 \\ 1423 & 14: 34: 38 & 51410.0 & 1424 & 14: 34: 44 & 51413.3 \\ 1425 & 14: 34: 48 & 51409.8 & 1426 & 14: 34: 53 & 51413.1 \\ 1427 & 14: 34: 56 & 51409.9 & 1428 & 14: 35: 02 & 51413.5 \\ 1429 & 14: 35: 05 & 51409.7 & 1514 & 14: 45: 25 & 51419.7 \\ 1515 & 14: 45: 29 & 51407.5 & 1516 & 14: 45: 34 & 51419.7 \\ 1517 & 14: 45: 37 & 51407.6 & 1518 & 14: 45: 43 & 51418.5 \\ 1519 & 14: 45: 46 & 51407.2 & 1520 & 14: 45: 51 & 51418.5 \\ 1521 & 14: 45: 55 & 51407.2 & 1522 & 14: 46: 00 & 51418.5 \\ 1523 & 14: 46: 03 & 51407.0 & & & \end{array}$

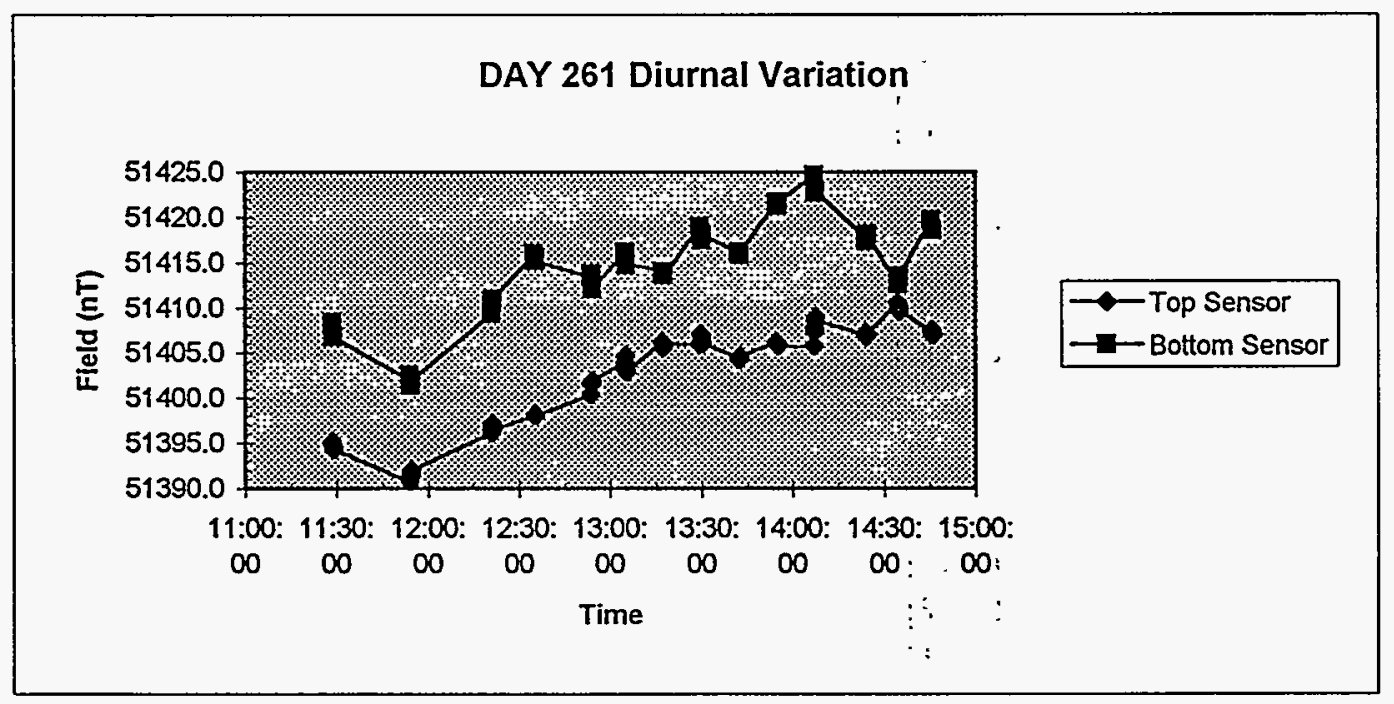


Hydroflouric Acid Spill Magnetic Survey Data

$\begin{array}{ccr} & \text { TOP } & \\ \text { Sta. } & \text { Time } & \text { Field (nT) } \\ 1555 & 12: 12: 17 & 51398.0 \\ 1557 & 12: 12: 27 & 51397.7 \\ 1559 & 12: 12: 38 & 51397.5 \\ 1561 & 12: 12: 49 & 51396.6 \\ 1646 & 12: 24: 29 & 51392.6 \\ 1648 & 12: 24: 40 & 51393.0 \\ 1650 & 12: 24: 51 & 51392.0 \\ 1652 & 12: 25: 02 & 51392.3 \\ 1654 & 12: 25: 12 & 51392.4 \\ 1734 & 12: 37: 17 & 51394.8 \\ 1736 & 12: 37: 28 & 51395.5 \\ 1738 & 12: 37: 38 & 51395.1 \\ 1740 & 12: 37: 49 & 51395.0 \\ 1742 & 12: 38: 00 & 51395.0 \\ 1818 & 12: 50: 42 & 51399.7 \\ 1820 & 12: 50: 54 & 51399.2 \\ 1822 & 12: 51: 04 & 51398.9 \\ 1824 & 12: 51: 16 & 51398.7 \\ 1826 & 12: 51: 28 & 51398.5 \\ 1898 & 13: 01: 27 & 51400.4 \\ 1900 & 13: 01: 39 & 51400.6 \\ 1902 & 13: 01: 50 & 51400.0 \\ 1904 & 13: 02: 02 & 51399.9 \\ 1906 & 13: 02: 13 & 51399.9 \\ 1978 & 13: 12: 39 & 51406.9 \\ 1980 & 13: 12: 50 & 51406.7 \\ 1982 & 13: 13: 01 & 51406.7 \\ 1984 & 13: 13: 11 & 51406.9 \\ 1986 & 13: 13: 22 & 51406.8 \\ 2056 & 13: 23: 52 & 51406.3 \\ 2058 & 13: 24: 03 & 51406.3 \\ 2060 & 13: 24: 13 & 51406.1 \\ 2062 & 13: 24: 24 & 51406.0 \\ 2064 & 13: 24: 35 & 51406.0 \\ 2130 & 13: 35: 12 & 51407.4 \\ 2132 & 13: 35: 24 & 51407.6 \\ 2134 & 13: 35: 36 & 51407.3 \\ 2136 & 13: 35: 48 & 51403.7 \\ 2138 & 13: 36: 01 & 51401.1 \\ 2204 & 13: 45: 42 & 51408.3 \\ 2206 & 13: 45: 53 & 51408.6 \\ 2208 & 13: 46: 04 & 51408.5 \\ 2210 & 13: 46: 15 & 51408.9 \\ 2212 & 13: 46: 25 & 51409.2\end{array}$

DAY 262 Diurnal Variation

\section{BOTTOM}

$\begin{array}{ccr}\text { Sta. } & \text { Time } & \text { Field (nT) } \\ 1554 & 12: 12: 13 & 51399.8 \\ 1556 & 12: 12: 23 & 51399.4 \\ 1558 & 12: 12: 34 & 51399.3 \\ 1560 & 12: 12: 44 & 51401.3 \\ 1645 & 12: 24: 25 & 51416.0 \\ 1647 & 12: 24: 36 . . & 51415.8 \\ 1649 & 12: 24: 46 & 51415.0 \\ 1651 & 12: 24: 57 & 51415.3 \\ 1653 & 12: 25: 08 & 51415.8 \\ 1733 & 12: 37: 13 & 51419.2 \\ 1735 & 12: 37: 23 & 51418.9 \\ 1737 & 12: 37: 34 & 51419.3 \\ 1739 & 12: 37: 45 & 51419.2 \\ 1741 & 12: 37: 56 & 51420.0 \\ 1817 & 12: 50: 37 & 51404.9 \\ 1819 & 12: 50: 49 & 51405.0 \\ 1821 & 12: 51: 00 & 51405.1 \\ 1823 & 12: 51: 12 & 51405.5 \\ 1825 & 12: 51: 23 & 51405.2 \\ 1897 & 13: 01: 23 & 51413.2 \\ 1899 & 13: 01: 34 & 51414.1 \\ 1901 & 13: 01: 46 & 51414.2 \\ 1903 & 13: 01: 57 & 51414.0 \\ 1905 & 13: 02: 09 & 51414.0 \\ 1977 & 13: 12: 35 & 51426.3 \\ 1979 & 13: 12: 45 & 51426.8 \\ 1981 & 13: 12: 56 & 51427.5 \\ 1983 & 13: 13: 07 & 51427.9 \\ 1985 & 13: 13: 18 & 51428.0 \\ 2055 & 13: 23: 48 & 51426.1 \\ 2057 & 13: 23: 58 & 51425.7 \\ 2059 & 13: 24: 09 & 51425.8 \\ 2061 & 13: 24: 20 & 51425.6 \\ 2063 & 13: 24: 31 & 51426.1 \\ 2129 & 13: 35: 08 & 51422.2 \\ 2131 & 13: 35: 20 & 51422.7 \\ 2133 & 13: 35: 31 & 51423.2 \\ 2135 & 13: 35: 44 & 51426.3 \\ 2137 & 13: 35: 57, & 51426.4 \\ 2203 & 13: 45: 38 & 51414.7 \\ 2205 & 13: 45: 49 & 51413.5 \\ 2207 & 13: 46: 00 & 51413.7 \\ 2209 & 13: 46: 10 & 51413.8 \\ 2211 & 13: 46: 21 & 51414.1\end{array}$


Hydroflouric Acid Spill Magnetic Survey Data

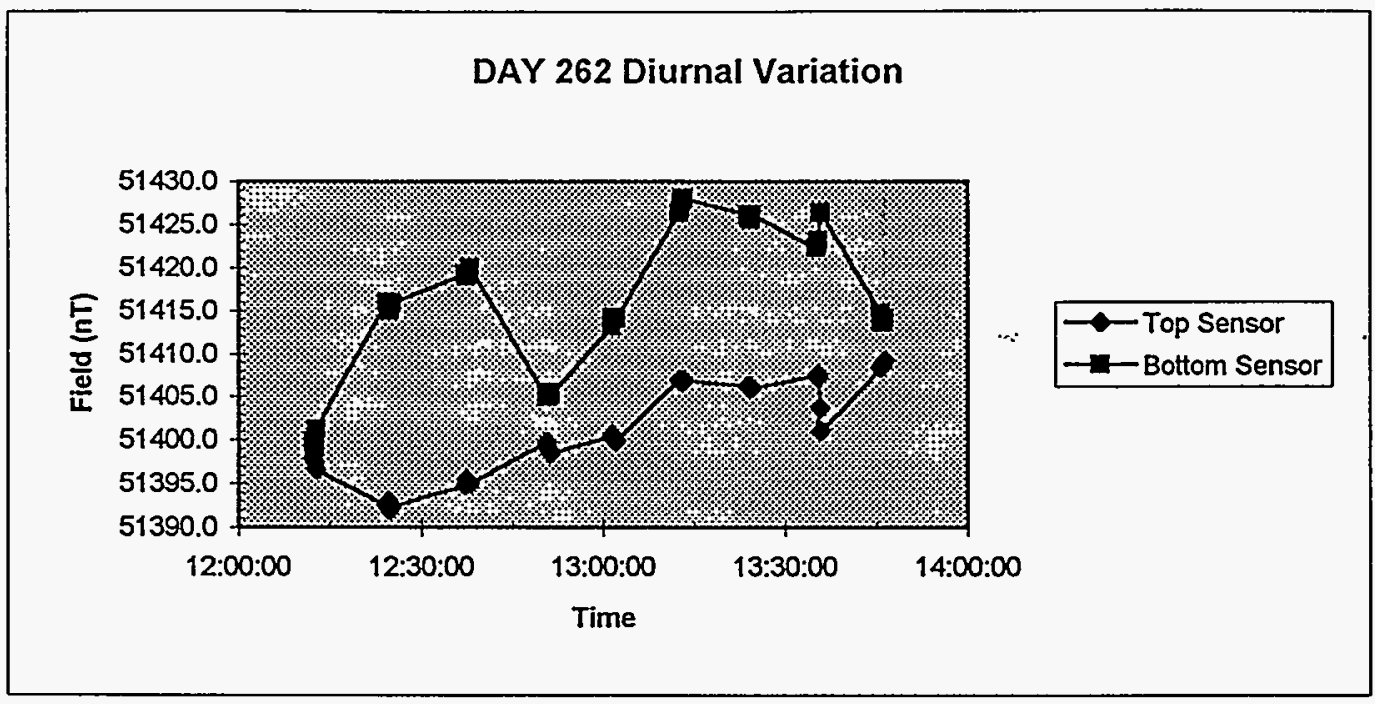

$\begin{array}{rcr}\text { Sta. } & \text { TOP } & \\ 1 & \text { Time } & \text { Field }(\mathbf{n T}) \\ 3 & 8: 38: 40 & 51381.0 \\ 5 & 8: 38: 53 & 51381.0 \\ 7 & 8: 39: 00 & 51381.0 \\ 9 & 8: 39: 06 & 51380.4 \\ 131 & 8: 51: 53 & 51379.4 \\ 133 & 8: 52: 00 & 51377.8 \\ 135 & 8: 52: 07 & 51377.8 \\ 137 & 8: 52: 15 & 51378.0 \\ 139 & 8: 52: 21 & 51377.6 \\ 405 & 9: 34: 39 & 51384.6 \\ 407 & 9: 34: 46 & 51384.2 \\ 409 & 9: 34: 52 & 51384.8 \\ 411 & 9: 34: 58 & 51384.8 \\ 413 & 9: 35: 04 & 51385.0\end{array}$

DAY 264Diurnal Variation̈

\begin{tabular}{ccr}
\multicolumn{3}{c}{ BOTTOM } \\
Sta. & Time & Field (nT) \\
0 & $8: 38: 38$ & 51395.0 \\
2 & $8: 38: 45$ & 51394.8 \\
4 & $8: 38: 51$ & 51395.2 \\
6 & $8: 38: 58$ & 51394.6 \\
8 & $8: 39: 04$ & 51393.8 \\
130 & $8: 51: 51$ & 51383.8 \\
132 & $8: 51: 58$ & 51384.4 \\
134 & $8: 52: 04$ & 51385.0 \\
136 & $8: 52: 13$ & 51385.2 \\
138 & $8: 52: 19$ & 51384.8 \\
404 & $9: 34: 37$ & 51397.6 \\
406 & $9: 34: 43$ & 51396.2 \\
408 & $9: 34: 50$ & 51396.4 \\
410 & $9: 34: 56$ & 51394.8 \\
412 & $9: 35: 02$ & 51394.2
\end{tabular}

\section{DAY 264 Diurnal Variation}
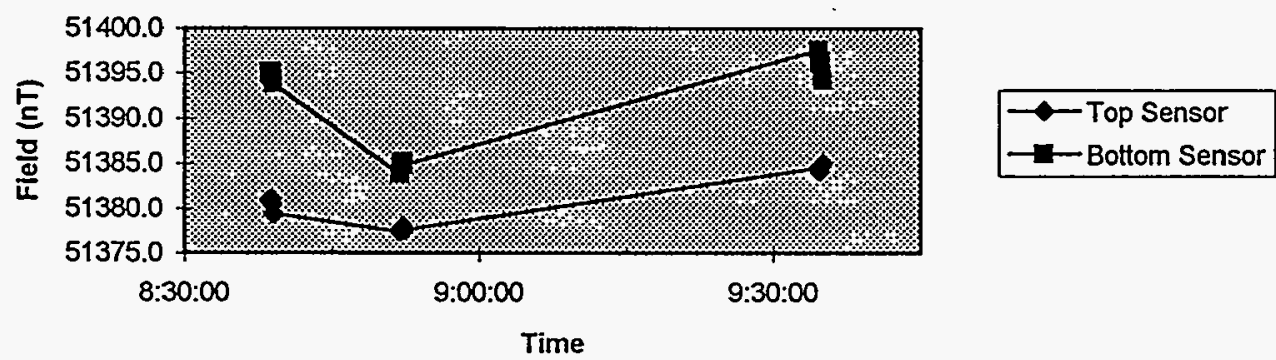
Hydroflouric Acid Spill Magnetic Survey Data

* represents missing data due to high magnetic gradients

TOP

BOTTOM VERT.

LINE DAY TIME STA. $X \quad Y$

$\begin{array}{llllll}0 & 261 & 112946 & 10 & 0 & 0 \\ 0 & 261 & 112957 & 12 & 0 & -1 \\ 0 & 261 & 113009 & 14 & 0 & -2 \\ 0 & 261 & 113021 & 16 & 0 & -3 \\ 0 & 261 & 113044 & 18 & 0 & -4 \\ 0 & 261 & 113054 & 20 & 0 & -5 \\ 0 & 261 & 113105 & 22 & 0 & -6 \\ 0 & 261 & 113115 & 24 & 0 & -7 \\ 0 & 261 & 113124 & 26 & 0 & -8 \\ 0 & 261 & 113134 & 28 & 0 & -9 \\ 0 & 261 & 113146 & 30 & 0 & -10 \\ 0 & 261 & 113156 & 32 & 0 & -11 \\ 0 & 261 & 113208 & 34 & 0 & -12 \\ 0 & 261 & 113219 & 36 & 0 & -13 \\ 0 & 261 & 113229 & 38 & 0 & -14 \\ 0 & 261 & 113238 & 40 & 0 & -15 \\ 0 & 261 & 113248 & 42 & 0 & -16 \\ 0 & 261 & 113258 & 44 & 0 & -17 \\ 0 & 261 & 113307 & 46 & 0 & -18 \\ 0 & 261 & 113317 & 48 & 0 & -19 \\ 0 & 261 & 113326 & 50 & 0 & -20 \\ 0 & 261 & 113335 & 52 & 0 & -21 \\ 0 & 261 & 113344 & 54 & 0 & -22 \\ 0 & 261 & 113354 & 56 & 0 & -23 \\ 0 & 261 & 113404 & 58 & 0 & -24 \\ 0 & 261 & 113412 & 60 & 0 & -25 \\ 0 & 261 & 113641 & 62 & 0 & -26 \\ 0 & 261 & 113651 & 64 & 0 & -27 \\ 0 & 261 & 113701 & 66 & 0 & -28 \\ 0 & 261 & 113711 & 68 & 0 & -29 \\ 0 & 261 & 113719 & 70 & 0 & -30 \\ 0 & 261 & 113729 & 72 & 0 & -31 \\ 0 & 261 & 113739 & 74 & 0 & -32 \\ 0 & 261 & 113749 & 76 & 0 & -33 \\ 0 & 261 & 113759 & 78 & 0 & -34 \\ 0 & 261 & 113809 & 80 & 0 & -35 \\ 0 & 261 & 113819 & 82 & 0 & -36 \\ 0 & 261 & 113829 & 84 & 0 & -37 \\ 0 & 261 & 113838 & 86 & 0 & -38 \\ 0 & 261 & 113847 & 88 & 0 & -39 \\ 0 & 261 & 113858 & 90 & 0 & -40 \\ 0 & 261 & 113908 & 92 & 0 & -41 \\ 0 & 261 & 113918 & 94 & 0 & -42 \\ 0 & 261 & 113929 & 96 & 0 & -43 \\ 0 & 261 & 113940 & 98 & 0 & -44 \\ 0 & 261 & 113950 & 100 & 0 & -45 \\ 0 & 261 & 114000 & 102 & 0 & -46 \\ 0 & 261 & 114011 & 104 & 0 & -47\end{array}$

uncorr. corr. dif. uncorr. corr.

$\begin{array}{llllll}49775.6 & 49776.5 & -0.9 & 48818.6 & 48820.3 & -1.7\end{array}$

$\begin{array}{lllllll}50534.4 & 50535.3 & -0.9 & 50025.0 & 50026.8 & -1.8\end{array}$

$\begin{array}{lllllll}50971.6 & 50972.6 & -1.0 & 50807.2 & 50809.0 & -1.8\end{array}$

$\begin{array}{llllll}51164.4 & 51165.4 & -1.0 & 51094.2 & 51096.0 & -1.8\end{array}$

$\begin{array}{lllllll}51252.4 & 51253.4 & -1.0 & 51211.4 & 51213.3 & -1.9\end{array}$

$\begin{array}{lllllll}51298.8 & 51299.9 & -1.1 & 51267.6 & 51269.6 & -2.0\end{array}$

$\begin{array}{lllllll}51320.2 & 51321.3 & -1.1 & 51296.6 & 51298.6 & -2.0\end{array}$

$\begin{array}{lllllll}51321.6 & 51322.7 & -1.1 & 51298.2 & 51300.2 & -2.0\end{array}$

$\begin{array}{lllllll}51307.0 & 51308.1 & -1.1 & 51267.4 & 51269.5 & -2.1\end{array}$

$\begin{array}{lllllll}51256.8 & 51258.0 & -1.2 & 51174.0 & 51176.1 & -2.1\end{array}$

$\begin{array}{llllll}51213.0 & 51214.2 & -1.2 & 50968.4 & 50970.5 & -2.1\end{array}$

$\begin{array}{lllllll}51304.8 & 51306.0 & -1.2 & 50567.8 & 50570.0 & -2.2\end{array}$

$\begin{array}{lllllll}51791.8 & 51793.0 & -1.2 & 50467.0 & 50469.2 & -2.2\end{array}$

$\begin{array}{lllllll}51640.0 & 51641.3 & -1.3 & 50698.2 & 50700.4 & -2.2\end{array}$

$\begin{array}{lllllll}51454.8 & 51456.1 & -1.3 & 51044.6 & 51046.9 & -2.3\end{array}$

$\begin{array}{lllllll}51388.6 & 51389.9 & -1.3 & 51253.8 & 51256.1 & -2.3\end{array}$

$\begin{array}{lllllll}51385.2 & 51386.5 & -1.3 & 51327.4 & 51329.7 & -2.3\end{array}$

$\begin{array}{lllllll}51390.8 & 51392.2 & -1.4 & 51362.6 & 51364.9 & -2.3\end{array}$

$\begin{array}{lllllll}51401.2 & 51402.6 & -1.4 & 51385.2 & 51387.6 & -2.4\end{array}$

$\begin{array}{lllllll}51405.4 & 51406.8 & -1.4 & 51396.0 & 5,1398.4 & -2.4\end{array}$

$\begin{array}{lllllll}51407.4 & 51408.8 & -1.4 & 51395.6 & 51398.0 & -2.4\end{array}$

$\begin{array}{lllllll}51407.4 & 51408.9 & -1.5 & 51392.6 & 51395.1 & -2.5\end{array}$

$\begin{array}{lllllll}51406.4 & 51407.9 & -1.5 & 51395.8 & 51398.3 & -2.5\end{array}$

$\begin{array}{llllll}51406.2 & 51407.7 & -1.5 & 51396.0 & 51398.5 & -2.5\end{array}$

$\begin{array}{llllll}51403.6 & 51405.1 & -1.5 & 51393.2 & 51395.8 & -2.6\end{array}$

$\begin{array}{lllllll}51400.6 & 51402.1 & -1.5 & 51391.8 & 51394.4 & -2.6\end{array}$

$\begin{array}{lllllll}51400.0 & 51401.9 & -1.9 & 51391.2 & 51394.3 & -3.1\end{array}$

$\begin{array}{lllllll}51398.2 & 51400.1 & -1.9 & 513189.8 & 51392.9 & -3.1\end{array}$

$\begin{array}{lllllll}51394.6 & 51396.5 & -1.9 & 51385.8 & 51388.9 & -3.1\end{array}$

$\begin{array}{lllllll}51389.0 & 51391.0 & -2.0 & 51380.8 & 51384.0 & -3.2\end{array}$

$\begin{array}{lllllll}51384.6 & 51386.6 & -2.0 & 51373.6 & 51376.8 & -3.2\end{array}$

$\begin{array}{llllll}51381.2 & 51383.2 & -2.0 & 51373.8 & 51377.0 & -3.2\end{array}$

$\begin{array}{lllllll}51379.2 & 51381.2 & -2.0 & 51370.6 & 51373.8 & -3.2\end{array}$

$\begin{array}{lllllll}51377.4 & 51379.5 & -2.1 & 51366.6 & 51369.9 & -3.3\end{array}$

$\begin{array}{lllllll}51369.2 & 51371.3 & -2.1 & 513157.2 & 51360.5 & -3.3\end{array}$

$\begin{array}{lllllll}51367.0 & 51369.1 & -2.1 & 51359.8 & 51363.1 & -3.3\end{array}$

$\begin{array}{lllllll}51366.8 & 51368.9 & -2.1 & 51362.2 & 51365.6 & -3.4\end{array}$

$\begin{array}{lllllll}51358.0 & 51360.2 & -2.2 & 51354.4 & 51357.8 & -3.4\end{array}$

$\begin{array}{lllllll}51350.6 & 51352.8 & -2.2 & 51349.4 & 51352.8 & -3.4\end{array}$

$\begin{array}{lllllll}51345.0 & 51347.2 & -2.2 & 51340.6 & 51344.1 & -3.5\end{array}$

$\begin{array}{lllllll}51336.4 & 51338.6 & -2.2 & 51335.0 & 51338.5 & -3.5\end{array}$

$\begin{array}{llllll}51326.8 & 51329.1 & -2.3 & 51315.0 & 51318.5 & -3.5\end{array}$

$\begin{array}{lllllll}51314.6 & 51316.9 & -2.3 & 51307.0 & 51310.6 & -3.6\end{array}$

$\begin{array}{lllllll}51308.8 & 51311.1 & -2.3 & 51295.8 & 51299.4 & -3.6\end{array}$

$\begin{array}{lllllll}51301.2 & 51303.5 & -2.3 & 51292.0 & 51295.6 & -3.6\end{array}$

$\begin{array}{lllllll}51295.0 & 51297.4 & -2.4 & 51288.4 & 51292.1 & -3.7\end{array}$

$\begin{array}{lllllll}51296.4 & 51298.8 & -2.4 & 51284.4 & 51288.1 & -3.7\end{array}$

$\begin{array}{lllllll}51300.0 & 51302.4 & -2.4 & 51286.6 & 51290.3 & -3.7\end{array}$
RAD.(nT/m)

$-1568.9$

$-835.1$

$-269.5$

$-115.1$

$-67.2$

$-51.1$

$-38.7$

$-38.4$

$-64.9$

$-135.7$

$-401.0$

$-1208.2$

$-2171.8$

$-1543.9$

$-672.5$

$-221.0$

$-94.7$

$-46.2$

$-26.2$

$-15.4$

$-19.3$

$-24.3$

$-17.4$

$-16.7$

$-17.1$

$-14.4$

$-14.4$

$-13.8$

$-14.4$

$-13.4$

$-18.0$

$-12.1$

$-14.1$

$-17.7$

$-19.7$

$-11.8$

$-7.5$

$-5.9$

$-2.0$

$-7.2$

$-2.3$

$-19.3$

$-12.5$

$-21.3$

$-15.1$

$-10.8$

$-19.7$

$-22.0$ 
Hydroflouric Acid Spill Magnetic Survey Data

LINE DAY TIME STA. $\mathbf{X}$ $261114021 \quad 106$ $\begin{array}{lll}261 & 114033 & 108\end{array}$

$\begin{array}{lll}261 & 114043 \quad 110\end{array}$

$\begin{array}{llll}261 & 114052 & 112 & 0\end{array}$

$\begin{array}{lll}261 & 114305 & 114\end{array}$

$\begin{array}{llll}261 & 114316 & 116\end{array}$

$\begin{array}{lll}261 & 114326 & 118\end{array}$

$\begin{array}{lll}261 & 114335 & 120\end{array}$

$\begin{array}{lll}261 & 114347 & 122\end{array}$

$\begin{array}{llll}261 & 114357 & 124\end{array}$

$\begin{array}{llll}261 & 114407 & 126\end{array}$

$\begin{array}{llll}261 & 114416 & 128\end{array}$

$\begin{array}{lll}261 & 114426 & 130\end{array}$

$\begin{array}{llll}261 & 114437 & 132 & 1\end{array}$

$\begin{array}{lll}261 & 114447 & 134\end{array}$

$\begin{array}{lll}261 & 114457 & 136\end{array}$

$\begin{array}{lll}261 & 114508 & 138\end{array}$

$\begin{array}{lll}261 & 114518 & 140\end{array}$

$\begin{array}{lll}261 & 114528 & 142\end{array}$

$\begin{array}{lll}261 & 114537 & 144\end{array}$

$\begin{array}{lll}261 & 114546 & 146\end{array}$

$\begin{array}{lll}261 & 114555 & 148\end{array}$

$\begin{array}{lll}261 & 114605 & 150\end{array}$

$\begin{array}{lll}261 & 114614 & 152\end{array}$

$\begin{array}{lll}261 & 114625 & 154\end{array}$

$\begin{array}{lll}261 & 114635 & 156\end{array}$

$\begin{array}{lll}261 & 114644 & 158\end{array}$

$\begin{array}{lll}261 & 114654 & 160\end{array}$

$\begin{array}{lll}261 & 114704 & 162\end{array}$

$\begin{array}{lll}261 & 114714 & 164\end{array}$

$\begin{array}{lll}261 & 114900 & 166\end{array}$

$\begin{array}{lll}261 & 114910 & 168\end{array}$

$\begin{array}{lll}261 & 114920 & 170\end{array}$

$\begin{array}{lll}261 & 114930 & 172\end{array}$

$\begin{array}{lll}261 & 114940 & 174\end{array}$

$\begin{array}{lll}261 & 114951 & 176\end{array}$

$\begin{array}{llll}261 & 115001 & 178\end{array}$

$\begin{array}{lll}261 & 115011 & 180\end{array}$

$\begin{array}{lll}261 & 115021 & 182\end{array}$

$\begin{array}{lll}261 & 115031 & 184\end{array}$

$\begin{array}{llll}261 & 115041 & 186\end{array}$

$\begin{array}{lll}261 & 115050 & 188\end{array}$

$\begin{array}{lll}261 & 115100 & 190\end{array}$

$\begin{array}{lll}261 & 115111 & 192\end{array}$

$\begin{array}{lll}261 & 115121 & 194\end{array}$

$\begin{array}{lll}261 & 115131 & 196\end{array}$

$\begin{array}{lll}261 & 115143 & 198\end{array}$

$\begin{array}{lll}261 & 115153 & 200\end{array}$

$\begin{array}{lll}261 & 115203 \quad 202\end{array}$
$Y$

$-48$

$-49$

$-50$

$-51$

0

$-1$

$-2$

$-3$

$-4$

$-5$

$-6$

$-7$

$-8$

$-9$

$-10$

$-11$

$-12$

$-13$

$-14$

$-15$

$-16$

$-17$

$-18$

$-19$

$-20$

$-21$

$-22$

$-23$

$-24$

$-25$

$-26$

$-27$

$-28$

$-29$

$-30$

$-31$

$-32$

$-33$

$-34$

$-35$

$-36$

$-37$

$-38$

$-39$

$-40$

$-41$

$-42$

$-43$

$-44$
TOP

- BOTTOM

uncorr. corr. dif. uncorr. corr.

$\begin{array}{llllll}51306.8 & 51309.2 & -2.4 & 51299.0 & 51302.8 & -3.8\end{array}$

$\begin{array}{lllllll}51324.2 & 51326.7 & -2.5 & 51307.6 & 51311.4 & -3.8\end{array}$

$\begin{array}{lllllll}51348.8 & 51351.3 & -2.5 & 51323.4 & 51327.2 & -3.8\end{array}$

$\begin{array}{lllllll}51417.4 & 51419.9 & -2.5 & 51422.6 & 51426.5 & -3.9\end{array}$

$50697.8 \quad 50700.6-2.8$

$\begin{array}{lllllll}50376.8 & 50379.6 & -2.8 & 49672.4 & 49676.7 & -4.3\end{array}$

$\begin{array}{lllllll}50912.0 & 50914.9 & -2.9 & 50711.4 & 50715.8 & -4.4\end{array}$

$\begin{array}{lllllll}51154.4 & 51157.3 & -2.9 & 51071.0 & 51075.4 & -4.4\end{array}$

$\begin{array}{lllllll}51249.2 & 51252.1 & -2.9 & 51208.0 & 51212.4 & -4.4\end{array}$

$\begin{array}{lllllll}51296.8 & 51299.7 & -2.9 & 51268.0 & 51272.5 & -4.5\end{array}$

$\begin{array}{lllllll}51320.2 & 51323.2 & -3.0 & 51296.2 & 51300.7 & -4.5\end{array}$

$\begin{array}{lllllll}51317.4 & 51320.4 & -3.0 & 51294.0 & 51298.5 & -4.5\end{array}$

$\begin{array}{lllllll}51286.0 & 51289.0 & -3.0 & 51247.6 & 51252.2 & -4.6\end{array}$

$\begin{array}{lllllll}51206.8 & 51209.8 & -3.0 & 51111.0 & 51115.6 & -4.6\end{array}$

$\begin{array}{lllllll}51088.6 & 51091.7 & -3.1 & 50654.0 & 50658.6 & -4.6\end{array}$

$51969.6 \quad 51972.7 \quad-3.1$

$52408.4 \quad 52411.5-3.1$

$\begin{array}{lllllll}58643.8 & 58646.9 & -3.1 & 58611.0 & 58615.7 & -4.7\end{array}$

$\begin{array}{lllllll}51763.6 & 51766.8 & -3.2 & 51550.8 & 51555.6 & -4.8\end{array}$

$\begin{array}{lllllll}51414.0 & 51417.2 & -3.2 & 51219.8 & 51224.6 & -4.8\end{array}$

$\begin{array}{lllllll}51375.8 & 51379.0 & -3.2 & 51298.2 & 51303.0 & -4.8\end{array}$

$\begin{array}{lllllll}51381.0 & 51384.2 & -3.2 & 51342.8 & 51347.6 & -4.8\end{array}$

$\begin{array}{lllllll}51390.6 & 51393.9 & -3.3 & 51372.2 & 51377.1 & -4.9\end{array}$

$\begin{array}{lllllll}51405.6 & 51408.9 & -3.3 & 51392.2 & 51397.1 & -4.9\end{array}$

$\begin{array}{lllllll}51407.8 & 51411.1 & -3.3 & 51394.2 & 51399.1 & -4.9\end{array}$

$\begin{array}{lllllll}51406.4 & 51409.7 & -3.3 & 51390.6 & 51395.6 & -5.0\end{array}$

$\begin{array}{lllllll}51407.4 & 51410.7 & -3.3 & 51390.2 & 51395.2 & -5.0\end{array}$

$\begin{array}{llllll}51404.2 & 51407.6 & -3.4 & 51389.4 & 51394.4 & -5.0\end{array}$

$\begin{array}{lllllll}51401.4 & 51404.8 & -3.4 & 51389.6 & 51394.7 & -5.1\end{array}$

$\begin{array}{llllll}51398.0 & 51401.4 & -3.4 & 51387.2 & 51392.3 & -5.1\end{array}$

$\begin{array}{llllll}51400.4 & 51404.1 & -3.7 & 51388.2 & 51393.6 & -5.4\end{array}$

$\begin{array}{llllll}51396.2 & 51399.9 & -3.7 & 51389.0 & 51394.5 & -5.5\end{array}$

$\begin{array}{llllll}51390.0 & 51393.7 & -3.7 & 51385.8 & 51391.3 & -5.5\end{array}$

$\begin{array}{lllllll}51390.2 & 51393.9 & -3.7 & 51380.8 & 51386.3 & -5.5\end{array}$

$\begin{array}{llllll}51385.8 & 51389.6 & -3.8 & 51378.8 & 51384.4 & -5.6\end{array}$

$\begin{array}{lllllll}51382.8 & 51386.6 & -3.8 & 51378.4 & 51384.0 & -5.6\end{array}$

$\begin{array}{lllllll}51376.6 & 51380.4 & -3.8 & 51371.0 & 51376.6 & -5.6\end{array}$

$\begin{array}{lllllll}51370.8 & 51374.6 & -3.8 & 51365.0 & 51370.7 & -5.7\end{array}$

$\begin{array}{lllllll}51365.4 & 51369.3 & -3.9 & 51348.0 & 51353.7 & -5.7\end{array}$

$\begin{array}{lllllll}51364.8 & 51368.7 & -3.9 & 51380.0 & 51385.7 & -5.7\end{array}$

$\begin{array}{lllllll}51362.0 & 51365.9 & -3.9 & 51365.0 & 51370.8 & -5.8\end{array}$

$\begin{array}{lllllll}51355.2 & 51359.1 & -3.9 & 51351.6 & 51357.4 & -5.8\end{array}$

$\begin{array}{lllllll}51344.0 & 51348.0 & -4.0 & 51344.8 & 51350.6 & -5.8\end{array}$

$\begin{array}{lllllll}51338.6 & 51342.6 & -4.0 & 51333.0 & 51338.8 & -5.8\end{array}$

$\begin{array}{lllllll}51328.4 & 51332.4 & -4.0 & 51325.6 & 51331.5 & -5.9\end{array}$

$\begin{array}{lllllll}51317.4 & 51321.4 & -4.0 & 51309.2 & 51315.1 & -5.9\end{array}$

$\begin{array}{lllllll}51302.4 & 51306.5 & -4.1 & 51296.6 & 51302.6 & -6.0\end{array}$

$\begin{array}{lllllll}51295.0 & 51299.1 & -4.1 & 51283.0 & 51289.0 & -6.0\end{array}$

$\begin{array}{llllll}51284.0 & 51288.1 & -4.1 & 51274.2 & 51280.2 & -6.0\end{array}$
VERT.

GRAD.(nT/m)

$-12.8$

$-27.2$

$-41.6$

8.5

$-1154.8$

$-328.8$

$-136.7$

$-67.5$

$-47.2$

$-39.3$

$-38.4$

$-62.9$

$-157.1$

$-712.5$

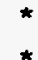

$-53.8$

$-348.9$

$-318.4$

$-127.2$

$-62.6$

$-30.2$

$-22.0$

$-22.3$

$-25.9$

$-28.2$

$-24.3$

$-19.3$

$-17.7$

$-20.0$

$-11.8$

$-6.9$

$-15.4$

$-11.5$

$-7.2$

$-9.2$

$-9.5$

$-28.5$

24.9

4.9

$-5.9$

1.3

$-9.2$

$-4.6$

$-13.4$

$-9.5$

$-19.7$

$-16.1$ 
Hydroflouric Acid Spill Magnetic Survey Data

TOP

BOTTOM

VERT.

LINE DAY TIME STA. $X$ $Y$ uncorr. corr. dif. uncorr. corr. dif. GRAD.(nT/m)

\begin{tabular}{|c|c|c|c|c|c|c|c|c|c|c|c|}
\hline 261 & 115212 & 204 & 1 & -45 & 51271.0 & 51275.1 & -4.1 & 51266.6 & 51272.6 & -6.0 & -7.2 \\
\hline 261 & 115223 & 206 & 1 & -46 & 51266.6 & 51270.8 & -4.2 & 51258.6 & 51264.7 & -6.1 & -13.1 \\
\hline 261 & 115233 & 208 & & -47 & 51267.4 & 51271.6 & -4.2 & 51256.8 & 51262.9 & -6.1 & -17.4 \\
\hline 261 & 115244 & 210 & & -48 & 51270.0 & 51274.2 & -4.2 & 51256.6 & 51262.7 & -6.1 & -22.0 \\
\hline 261 & 115254 & 212 & & -49 & 51287.2 & 51291.4 & -4.2 & 51275.8 & 51282.0 & -6.2 & -18.7 \\
\hline 261 & 115304 & 214 & & -50 & 51332.0 & 51336.3 & -4.3 & 51319.0 & 51325.2 & -6.2 & -21.3 \\
\hline 261 & 115314 & 216 & 1 & -51 & 51394.4 & 51398.7 & -4.3 & 405.2 & 411.4 & -6.2 & 17.7 \\
\hline 261 & 115325 & 218 & & -52 & 84.8 & & -4.3 & 556.4 & 562.7 & -6.3 & 117.4 \\
\hline 261 & 115634 & 230 & & 0 & 48.8 & & -2.9 & & * & * & \\
\hline 261 & 115647 & 232 & & -1 & 50207.6 & & -2.9 & 49367.1 & 49372.9 & -5.8 & -1377. \\
\hline 261 & 115659 & 234 & 8 & -2 & 50850.8 & 3.6 & -2.8 & 50616.9 & 50622.6 & -5.7 & -383.4 \\
\hline 261 & 115710 & 236 & 2 & -3 & 51115.2 & 3.0 & -2.8 & 51015.8 & 51021.5 & -5.7 & -162.9 \\
\hline 261 & 115721 & 238 & 2 & -4 & 51246.1 & 248.9 & -2.8 & 51195.9 & 51201.5 & -5.6 & -82.3 \\
\hline 261 & 115732 & 240 & 2 & -5 & 51298.8 & 301.5 & -2.7 & 51262.9 & 51268.5 & -5.6 & -58.9 \\
\hline 261 & 115742 & 242 & 2 & -6 & 51323.0 & 51325.7 & -2.7 & 51292.9 & 51298.4 & -5.5 & -49.3 \\
\hline 261 & 115752 & 244 & 2 & -7 & 51321.1 & & -2.7 & 51296.9 & 51302.4 & -5.5 & -39.7 \\
\hline 261 & 115804 & 246 & 2 & -8 & 51291.7 & 5129 & -2.6 & 51255.5 & 51260.9 & -5.4 & -59.3 \\
\hline 61 & 115814 & 248 & 2 & -9 & 51204.1 & 51206.7 & -2.6 & 51123.2 & 51128.6 & -5.4 & -132.6 \\
\hline 261 & 115825 & 250 & 2 & -10 & 50986.9 & 50989.5 & -2.6 & 0608.6 & 50613.9 & -5.3 & 620.2 \\
\hline 261 & 115836 & 252 & 2 & -11 & 1045.2 & 047.8 & -2.6 & & & $\star$ & \\
\hline 261 & 115848 & 254 & 2 & -12 & $\star$ & $\star$ & $\bullet$ & * & 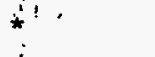 & ? & \\
\hline 261 & 115902 & 256 & 2 & -13 & * & * & • & $469 ๋ 29.2$ & 469 & -5.2 & \\
\hline 261 & 115913 & 258 & 2 & -14 & 45616.6 & 9.1 & -2.5 & & & $\approx$ & $\star$ \\
\hline 261 & 115925 & 260 & 2 & -15 & 43.1 & & -2.4 & 51605.7 & 51610.8 & -5.1 & 758.4 \\
\hline 261 & 115936 & 262 & 2 & -16 & 51362.2 & 64.6 & -2.4 & 51265.9 & 51270.9 & -5.0 & -157.9 \\
\hline 261 & 115948 & 264 & 2 & -17 & 51366.4 & & -2.4 & 05.7 & 51310.7 & -5.0 & -99.5 \\
\hline 261 & 115957 & 266 & 2 & -18 & 51385.2 & & -2.3 & 45.3 & 51350.2 & -4.9 & -65.4 \\
\hline 261 & 120008 & 268 & 2 & -19 & 51400.5 & 02.8 & -2.3 & 51378.7 & 51383.6 & -4.9 & -35.7 \\
\hline 261 & 120018 & 270 & 2 & -20 & 51409.4 & 51411.7 & -2.3 & 51393.4 & 51398.2 & -4.8 & -26.2 \\
\hline 61 & 120027 & 272 & 2 & -21 & 51409.3 & 51411.6 & -2.3 & 51386.1 & 51390.9 & -4.8 & -38.0 \\
\hline 61 & 120037 & 274 & 2 & -22 & 51407.9 & 51410.1 & -2.2 & 51388.2 & 51392.9 & -4.7 & -32.3 \\
\hline 261 & 120046 & 276 & 2 & -23 & 51406.6 & 51408.8 & -2.2 & 51386.0 & 51390.7 & -4.7 & -33.8 \\
\hline 61 & 120057 & 278 & 2 & -24 & 51403.5 & 51405.7 & -2.2 & 51385.1 & 51389.7 & -4.6 & -30.2 \\
\hline 261 & 120107 & 280 & 2 & -25 & 51400.2 & 51402.4 & -2.2 & 51384.5 & 51389.1 & -4.6 & -25.7 \\
\hline 261 & 120313 & 282 & 2 & -26 & 51399.9 & & -1.8 & 78.7 & 382.7 & -4.0 & -34.8 \\
\hline 261 & 120324 & 284 & 2 & -27 & 51396.3 & & -1.8 & 378.7 & 382.7 & -4.0 & -28.9 \\
\hline 261 & 120335 & 286 & 2 & -28 & 513 & & -1.8 & 81.3 & 85.2 & -3.9 & -20.5 \\
\hline 261 & 120347 & 288 & 2 & -29 & 4.5 & & -1.7 & 78.9 & 82.8 & -3.9 & -25.6 \\
\hline 261 & 120357 & 290 & 2 & -30 & 90.1 & & -1.7 & 73.6 & 51377.4 & -3.8 & -27.0 \\
\hline 261 & 120407 & 292 & 2 & -31 & 51385.2 & 386.9 & -1.7 & 365.8 & 51369.6 & -3.8 & -31.8 \\
\hline 261 & 120417 & 294 & 2 & -32 & 51377.3 & 51379.0 & -1.7 & 51364.0 & 51367.7 & -3.7 & -21.8 \\
\hline 261 & 120428 & 296 & 2 & -33 & 51375.4 & 51377.0 & -1.6 & 51361.6 & 51365.3 & -3.7 & -22.6 \\
\hline 261 & 120438 & 298 & 2 & -34 & 51367.1 & 51368.7 & -1.6 & 51349.3 & 51352.9 & -3.6 & -29.2 \\
\hline 261 & 120449 & 300 & 2 & -35 & 51366.0 & 51367.6 & -1.6 & 51344.5 & 51348.1 & -3.6 & -35.2 \\
\hline 261 & 120500 & 302 & 2 & -36 & 51357.2 & 51358.7 & -1.5 & 51338.4 & 5134 & -3.5 & -30.8 \\
\hline 261 & 120510 & 304 & 2 & -37 & 51348.1 & 51349.6 & -1.5 & 51329.2 & 51332.7 & -3.5 & -31.0 \\
\hline 261 & 120520 & 306 & 2 & -38 & 51342.1 & 343.6 & -1.5 & 51322.1 & 51325.5 & -3.4 & -32.8 \\
\hline 261 & 120530 & 308 & 2 & -39 & 51326.8 & 51328.3 & -1.5 & 51310.2 & 51313.6 & -3.4 & -27.2 \\
\hline 261 & 120541 & 310 & 2 & -40 & 51311.9 & 51313.3 & -1.4 & 51298.3 & 51301.6 & -3.3 & -22.3 \\
\hline
\end{tabular}


Hydroflouric Acid Spill Magnetic Survey Data

TOP

LINE DAY TIME STA. $\mathrm{x}$

$\begin{array}{lllll}2 & 261 & 120551 & 312 & 2\end{array}$

$\begin{array}{lllll}2 & 261 & 120601 & 314\end{array}$

$\begin{array}{lllll}2 & 261 & 120612 & 316 & 2\end{array}$

$\begin{array}{lllll}2 & 261 & 120623 & 318 & 2\end{array}$

$\begin{array}{lllll}2 & 261 & 120634 & 320 & 2\end{array}$

$\begin{array}{lllll}2 & 261 & 120645 & 322 & 2\end{array}$

$\begin{array}{lllll}2 & 261 & 120656 & 324 & 2\end{array}$

$\begin{array}{lllll}2 & 261 & 120706 & 326 & 2\end{array}$

$\begin{array}{lllll}2 & 261 & 120717 & 328 & 2\end{array}$

$\begin{array}{lllll}2 & 261 & 120727 & 330 & 2\end{array}$

$\begin{array}{lllll}2 & 261 & 120737 & 332 & 2\end{array}$

$\begin{array}{lllll}2 & 261 & 120749 & 334 & 2\end{array}$

$\begin{array}{lllll}3 & 261 & 120858 & 336\end{array}$

$\begin{array}{lllll}3 & 261 & 121017 & 338 & 3\end{array}$

$\begin{array}{lllll}3 & 261 & 121028 & 340\end{array}$

$\begin{array}{lllll}3 & 261 & 121039 & 342 & 3\end{array}$

$\begin{array}{llll}3 & 261 & 121049 & 344\end{array}$

3

3

3

3

3

3

3

3

3

3

3

3

3

3

3

3

3

3

3

3

3

3

3

3

3

3

3

3

3

3

3

3 $\begin{array}{llll}261 & 121059 & 346 & 3\end{array}$

$\begin{array}{lll}261 & 121109 & 348\end{array}$

$\begin{array}{lll}261 & 121120 & 350\end{array}$

$\begin{array}{lll}261 & 121129 & 352\end{array}$

261121139354

261121149356

261121200358

261121211360

261121222362

$\begin{array}{lll}261 & 121233 & 364\end{array}$

261121243366

$\begin{array}{lll}261 & 121253 \quad 368\end{array}$

$\begin{array}{lll}261 & 121303 \quad 370\end{array}$

$\begin{array}{lll}261 & 121313 & 372\end{array}$

$\begin{array}{lll}261 & 121324 & 374\end{array}$

$\begin{array}{lll}261 & 121335 & 376\end{array}$

$\begin{array}{lll}261 & 121345 \quad 378\end{array}$

$\begin{array}{lll}261 & 121356380\end{array}$

$\begin{array}{lll}261 & 121407 & 382\end{array}$

$\begin{array}{lll}261 & 121417 \quad 384\end{array}$

$\begin{array}{lll}261 & 121428 & 386\end{array}$

$\begin{array}{lll}261 & 121538 & 388\end{array}$

$\begin{array}{lll}261 & 121559390\end{array}$

$\begin{array}{lll}261 & 121609 & 392\end{array}$

261121620394

$\begin{array}{lll}261 & 121631 & 396\end{array}$

$\begin{array}{lll}261 & 121641398\end{array}$

$\begin{array}{lll}261 & 121652400\end{array}$

$\begin{array}{lll}261 & 121702 \quad 402\end{array}$

$\begin{array}{lll}261 & 121713 \quad 404\end{array}$

$\begin{array}{lll}261 & 121724 \quad 406\end{array}$

$\begin{array}{llll}261 & 121734 & 408\end{array}$
$Y$ uncorr. corr

\section{$-41$}

$-42$

$-43$

$-44$

$-45$

$-46$

$-47$

$-48$

$-49$

$-50$

$-51$

$-52$

0

$-1$

$-2$

$-3$

$-4$

$-5$

$-6$

$-7$

$-8$

$-9$

$-10$

$-11$

$-12$

$-13$

$-14$

$-15$

$-16$

$-17$

$-18$

$-19$

$-20$

$-21$

$-22$

$-23$

$-24$

$-25$

$-26$

$-27$

$-28$

$-29$

$-30$

$-31$

$-32$

$-33$

$-34$

$-35$

$-36$ uncorr. corr. dif. uncorr. corr.

$\begin{array}{llllll}51299.8 & 51301.2 & -1.4 & 51284.9 & 51288.2 & -3.3\end{array}$

$\begin{array}{lllllll}51286.2 & 51287.6 & -1.4 & 51269.7 & 51272.9 & -3.2\end{array}$

$\begin{array}{lllllll}51267.8 & 51269.1 & -1.3 & 51252.2 & 51255.4 & -3.2\end{array}$

$\begin{array}{lllllll}51243.4 & 51244.7 & -1.3 & 51232.8 & 51235.9 & -3.1\end{array}$

$\begin{array}{lllllll}51235.8 & 51237.1 & -1.3 & 51218.3 & 51221.4 & -3.1\end{array}$

$\begin{array}{lllllll}51216.8 & 51218.1 & -1.3 & 51200.8 & 51203.8 & -3.0\end{array}$

$\begin{array}{lllllll}51198.5 & 51199.7 & -1.2 & 51186.8 & 5.1189 .8 & -3.0\end{array}$

$\begin{array}{lllllll}51190.8 & 51192.0 & -1.2 & 51172.8 & 51175.7 & -2.9\end{array}$

$\begin{array}{lllllll}51206.6 & 51207.8 & -1.2 & 51182.9 & 51185.8 & -2.9\end{array}$

$\begin{array}{lllllll}51255.4 & 51256.5 & -1.1 & 51228.1 & 51230.9 & -2.8\end{array}$

$\begin{array}{lllllll}51412.8 & 51413.9 & -1.1 & 51411.3 & 51414.1 & -2.8\end{array}$

$\begin{array}{lllllll}51593.2 & 51594.3 & -1.1 & 51757.2 & 51759.9 & -2.7\end{array}$

$\begin{array}{llll}45576.5 & 45577.4 & -0.9\end{array}$

$\begin{array}{lllllll}50231.5 & 50232.2 & -0.7 & 49825.3 & 49827.3 & -2.0\end{array}$

$\begin{array}{lllllll}50882.9 & 50883.6 & -0.7 & 50739.7 & 50741.7 & -2.0\end{array}$

$\begin{array}{lllllll}51132.2 & 51132.8 & -0.6 & 51059.7 & 51061.6 & -1.9\end{array}$

$\begin{array}{lllllll}51247.7 & 51248.3 & -0.6 & 51197.5 & 51199.4 & -1.9\end{array}$

$\begin{array}{lllllll}51301.7 & 51302.3 & -0.6 & 51263.7 & 51265.6 & -1.9\end{array}$

$\begin{array}{lllllll}51325.6 & 51326.2 & -0.6 & 51294.9 & 51296.7 & -1.8\end{array}$

$\begin{array}{lllllll}51326.9 & 51327.4 & -0.5 & 51303.8 & 51305.6 & -1.8\end{array}$

$\begin{array}{lllllll}51305.6 & 51306.1 & -0.5 & 51278.0 & 51279.7 & -1.7\end{array}$

$\begin{array}{lllllll}51233.2 & 51233.7 & -0.5 & 51182.9 & 51184.6 & -1.7\end{array}$

$\begin{array}{lllllll}51079.3 & 51079.8 & -0.5 & 50898.6 & 50900.2 & -1.6\end{array}$

$\begin{array}{lllllll}50834.0 & 50834.4 & -0.4 & 49897.4 & 49899.0 & -1.6\end{array}$

$51156.751157 .1-0.4 \quad * \quad *$ *

$\begin{array}{lllllll}51343.7 & 51344.1 & -0.4 & 49788.6 & 49790.1 & -1.5\end{array}$

$\begin{array}{lllllll}51539.8 & 51540.1 & -0.3 & 50812.4 & 50813.8 & -1.4\end{array}$

$\begin{array}{lllllll}51361.6 & 51361.9 & -0.3 & 51210.1 & 51211.5 & -1.4\end{array}$

$\begin{array}{lllllll}51345.7 & 51346.0 & -0.3 & 51234.8 & 51236.1 & -1.3\end{array}$

$\begin{array}{lllllll}51355.9 & 51356.2 & -0.3 & 51284.6 & 51285.9 & -1.3\end{array}$

$\begin{array}{lllllll}51375.6 & 51375.8 & -0.2 & 51328.1 & 51329.3 & -1.2\end{array}$

$\begin{array}{lllllll}51395.2 & 51395.4 & -0.2 & 51371.9 & 51373.1 & -1.2\end{array}$

$\begin{array}{llllll}51404.2 & 51404.4 & -0.2 & 51383.9 & 51385.0 & -1.1\end{array}$

$\begin{array}{lllllll}51409.3 & 51409.4 & -0.1 & 51388.0 & 51389.1 & -1.1\end{array}$

$\begin{array}{lllllll}51407.7 & 51407.8 & -0.1 & 51394.6 & 51395.6 & -1.0\end{array}$

$\begin{array}{lllllll}51403.8 & 51403.9 & -0.1 & 51385.8 & 51386.8 & -1.0\end{array}$

$\begin{array}{lllllll}51401.5 & 51401.6 & -0.1 & 51380.8 & 51381.7 & -0.9\end{array}$

$\begin{array}{lllllll}51400.8 & 51400.8 & 0.0 & 51375.5 & 51376.4 & -0.9\end{array}$

$\begin{array}{lllllll}51395.2 & 51395.0 & 0.2 & 51377.9 & 51378.5 & -0.6\end{array}$

$\begin{array}{lllllll}51398.3 & 51398.1 & 0.2 & 51375.2 & 51375.7 & -0.5\end{array}$

$\begin{array}{lllllll}51396.4 & 51396.2 & 0.2 & 51373.5 & 51373.9 & -0.4\end{array}$

$\begin{array}{lllllll}51394.2 & 51393.9 & 0.3 & 51376.9 & 51377.3 & -0.4\end{array}$

$\begin{array}{lllllll}51395.8 & 51395.5 & 0.3 & 51378.0 & 51378.3 & -0.3\end{array}$

$\begin{array}{lllllll}51390.1 & 51389.8 & 0.3 & 51368.7 & 51369.0 & -0.3\end{array}$

$\begin{array}{lllllll}51383.0 & 51382.6 & 0.4 & 51360.0 & 51360.2 & -0.2\end{array}$

$\begin{array}{lllllll}51380.6 & 51380.2 & 0.4 & 51360.3 & 51360.5 & -0.2\end{array}$

$\begin{array}{lllllll}51376.0 & 51375.6 & 0.4 & 51354.5 & 51354.6 & -0.1\end{array}$

$\begin{array}{lllllll}51366.2 & 51365.8 & 0.4 & 51344.7 & 51344.8 & -0.1\end{array}$

$\begin{array}{llllll}51356.0 & 51355.5 & 0.5 & 51335.5 & 51335.5 & 0.0\end{array}$
VERT.

RAD.(nT/m)

$-24.4$

$-27.0$

$-25.6$

$-17.4$

$-28.7$

$-26.2$

$-19.2$

$-29.5$

$-38.9$

$-44.8$

$-2.5$

268.9

$-665.9$

$-234.8$

$-118.9$

$-82.3$

$-62.3$

$-50.3$

$-37.9$

$-45.2$

$-82.5$

$-296.2$

$-1535.4$

$-2549.3$

$-1192.5$

$-248.4$

$-181.8$

$-116.9$

$-77.9$

$-38.2$

$-33.3$

$-34.9$

$-21.5$

$-29.5$

$-33.9$

$-41.5$

$-28.4$

$-37.9$

$-37.5$

$-28.4$

$-29.2$

$-35.1$

$-37.7$

$-33.3$

$-35.2$

$-35.2$

$-33.6$ 
Hydroflouric Acid Spill Magnetic Survey Data

\begin{tabular}{|c|c|c|c|c|c|c|c|c|c|c|c|c|}
\hline & & & & & & & TOP & & BC & OTTOM & & VERT. \\
\hline & & & & $x$ & $y$ & & & dif. & & & dif. & GRAD.(nT/m) \\
\hline 3 & 261 & 121744 & 410 & 3 & -37 & 51344.2 & 51343.7 & 0.5 & 51326.3 & 51326.3 & 0.0 & -29.3 \\
\hline 3 & 261 & 121753 & 412 & 3 & -38 & 51332.7 & 51332.2 & 0.5 & 51318.1 & 51318.0 & 0.1 & -23.9 \\
\hline 3 & 261 & 121803 & 414 & 3 & -39 & 51322.6 & 51322.1 & 0.5 & 51308.2 & 51308.1 & 0.1 & -23.6 \\
\hline 3 & 261 & 121813 & 416 & 3 & -40 & 51307.6 & 51307.0 & 0.6 & 51294.5 & 51294.3 & 0.2 & -21.5 \\
\hline 3 & 261 & 121823 & 418 & 3 & -41 & 51294.4 & 51293.8 & 0.6 & 51278.5 & 51278.3 & 0.2 & -26.1 \\
\hline 3 & 261 & 121833 & 420 & 3 & -42 & 51272.4 & 51271.8 & 0.6 & 51261.6 & 51261.3 & 0.3 & -17.7 \\
\hline 3 & 261 & 121844 & 422 & 3 & -43 & 51257.2 & 51256.5 & 0.7 & 51238.8 & 51238.5 & 0.3 & -30.2 \\
\hline 3 & 261 & 121854 & 424 & 3 & -44 & 51230.2 & 51229.5 & 0.7 & 51214.8 & 51214.5 & 0.3 & -25.2 \\
\hline 3 & 261 & 121905 & 426 & 3 & -45 & 51200.8 & 51200.1 & 0.7 & 51187.9 & 51187.5 & 0.4 & -21.1 \\
\hline 3 & 261 & 121915 & 428 & 3 & -46 & 51172.2 & 51171.5 & 0.7 & 51160.3 & 51159.9 & 0.4 & -19.5 \\
\hline 3 & 261 & 121925 & 430 & 3 & -47 & 51136.5 & 51135.7 & 0.8 & 51127.0 & 51126.5 & 0.5 & -15.6 \\
\hline 3 & 261 & 121935 & 432 & 3 & -48 & 51102.7 & 51101.9 & 0.8 & 51095.1 & 51094.6 & 0.5 & -12.5 \\
\hline 3 & 261 & 121945 & 434 & 3 & -49 & 51083.5 & 51082.7 & 0.8 & 51070.8 & 51070.2 & 0.6 & -20.8 \\
\hline 3 & 261 & 121956 & 436 & 3 & -50 & 51122.2 & 51121.4 & 0.8 & 51087.7 & 51087.1 & 0.6 & -56.6 \\
\hline 3 & 261 & 122005 & 438 & 3 & -51 & 51220.6 & 51219.7 & 0.9 & 51164.1 & 51163.4 & 0.7 & -92.6 \\
\hline 3 & 261 & 122016 & 440 & 3 & -52 & 51559.4 & 51558.5 & 0.9 & 51566.2 & 51565.5 & 0.7 & 11.1 \\
\hline 4 & 261 & 122222 & 452 & 4 & 0 & 48933.8 & 48932.3 & 1.5 & 48614.6 & 48611.7 & 2.9 & -523.3 \\
\hline 4 & 261 & 122235 & 454 & 4 & -1 & 50350.5 & 50349.0 & 1.5 & 50052.1 & 50049.1 & 3.0 & -489.2 \\
\hline 4 & 261 & 122245 & 456 & 4 & -2 & 50897.7 & 50896.2 & 1.5 & 50773.6 & 50770.5 & 3.1 & -203.4 \\
\hline 4 & 261 & 122254 & 458 & 4 & -3 & 51142.5 & 51140.9 & 1.6 & 51070.5 & 5.1067 .4 & 3.1 & -118.0 \\
\hline 4 & 261 & 122305 & 460 & 4 & -4 & 51247.1 & 51245.5 & 1.6 & 51200.2 & 51197.0 & 3.2 & -76.9 \\
\hline 4 & 261 & 122315 & 462 & 4 & -5 & 51303.6 & 51302.0 & 1.6 & 51265.4 & 51262.1 & 3.3 & -62.6 \\
\hline 4 & 261 & 122326 & 464 & 4 & -6 & 51331.7 & 51330.1 & 1.6 & 51300.4 & 51297.1 & 3.3 & -51.3 \\
\hline 4 & 261 & 122336 & 466 & 4 & -7 & 51337.9 & 51336.3 & 1.6 & 51312.4 & 513009.0 & 3.4 & -41.8 \\
\hline 4 & 261 & 122347 & 468 & 4 & -8 & 51323.6 & 51321.9 & 1.7 & 513005.9 & 51302.4 & 3.5 & -29.0 \\
\hline 4 & 261 & 122357 & 470 & 4 & -9 & 51297.3 & 51295.6 & 1.7 & 51264.8 & 51261.3 & 3.5 & -53.3 \\
\hline 4 & 261 & 122407 & 472 & 4 & -10 & 51214.5 & 51212.8 & 1.7 & 51158.3 & 51154.7 & 3.6 & -92.1 \\
\hline 4 & 261 & 122418 & 474 & 4 & -11 & 51125.0 & 51123.3 & 1.7 & 50993.4 & 50989.8 & 3.6 & -215.7 \\
\hline 4 & 261 & 122429 & 476 & 4 & -12 & 51087.3 & 51085.6 & 1.7 & 50853.4 & 50849.7 & 3.7 & -383.4 \\
\hline 4 & 261 & 122439 & 478 & 4 & -13 & 51151.2 & 51149.4 & 1.8 & 50890.5 & 50886.7 & 3.8 & -427.4 \\
\hline 4 & 261 & 122449 & 480 & 4 & -14 & 51277.2 & 51275.4 & 1.8 & 51097.3 & 51093.5 & 3.8 & -294.9 \\
\hline 4 & 261 & 122458 & 482 & 4 & -15 & 51327.9 & 51326.1 & 1.8 & 51222.4 & 51218.5 & 3.9 & -173.0 \\
\hline 4 & 261 & 122508 & 484 & 4 & -16 & 51349.3 & 51347.5 & 1.8 & 51291.9 & 51287.9 & 4.0 & -94.1 \\
\hline 4 & 261 & 122519 & 486 & 4 & -17 & 51364.4 & 51362.6 & 1.8 & $513 ె 07 . \dot{7}$ & 51303.7 & 4.0 & -93.0 \\
\hline 4 & 261 & 122529 & 488 & 4 & -18 & 51381.1 & 51379.3 & 1.8 & $513 ̈ 33.1$ & 51329.0 & 4.1 & -78.7 \\
\hline 4 & 261 & 122538 & 490 & 4 & -19 & 51396.2 & 51394.3 & 1.9 & 513064.1 & 51360.0 & 4.1 & -52.6 \\
\hline 4 & 261 & 122548 & 492 & 4 & -20 & 51401.7 & 51399.8 & 1.9 & 51372.2 & 51368.0 & 4.2 & -48.4 \\
\hline 4 & 261 & 122558 & 494 & 4 & -21 & 51401.9 & 51400.0 & 1.9 & 51382.0 & 51377.7 & 4.3 & -32.6 \\
\hline 4 & 261 & 122607 & 496 & 4 & -22 & 51402.4 & 51400.5 & 1.9 & 51386.4 & 51382.1 & 4.3 & -26.2 \\
\hline 4 & 261 & 122617 & 498 & 4 & -23 & 51395.9 & 51394.0 & 1.9 & 51379.5 & 51375.1 & 4.4 & -26.9 \\
\hline 4 & 261 & 122627 & 500 & 4 & -24 & 51395.1 & 51393.1 & 2.0 & 51379.6 & 51375.2 & 4.4 & -25.4 \\
\hline 4 & 261 & 122638 & 502 & 4 & -25 & 51394.4 & 51392.4 & 2.0 & 51378.0 & 51373.5 & 4.5 & -26.9 \\
\hline 4 & 261 & 122739 & 504 & 4 & -26 & 51394.2 & 51392.1 & 2.1 & 51372.5 & 51367.6 & 4.9 & -35.6 \\
\hline 4 & 261 & 122749 & 506 & 4 & -27 & 51392.4 & 51390.3 & 2.1 & 51372.7 & 51367.8 & 4.9 & -32.3 \\
\hline 4 & 261 & 122758 & 508 & 4 & -28 & 51395.9 & 51393.8 & 2.1 & 51374.5 & 5 & 5.0 & -35.1 \\
\hline 4 & 261 & 122809 & 510 & 4 & -29 & 51388.6 & 51386.5 & 2.1 & 51374.6 & 51369.5 & 5.1 & -23.0 \\
\hline 4 & 261 & 122819 & 512 & 4 & -30 & 51390.1 & 51387.9 & 2.2 & 51369.3 & $5+364.2$ & 5.1 & -34.1 \\
\hline 4 & 261 & 122829 & 514 & 4 & -31 & 51382.0 & 51379.8 & 2.2 & 51368.2 & 51363.0 & 5.2 & -22.6 \\
\hline 4 & 261 & 122840 & 516 & 4 & -32 & 51378.3 & 51376.1 & 2.2 & 51361.2 & 51355.9 & 5.3 & -28.0 \\
\hline
\end{tabular}


TOP

$$
4
$$

4

4

\section{4} 4

\section{4}

\section{4}

LINE DAY TIME STA. $X$

-10
5
5

(2)

5
5
5
5

5
5
5
5
5

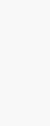

(5)

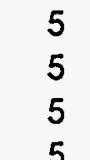

\begin{tabular}{lccccc} 
NE & DAY & TIME & STA. & X & Y \\
\hline & 261 & 122855 & 518 & 4 & -33 \\
4 & 261 & 122905 & 520 & 4 & -34 \\
4 & 261 & 122915 & 522 & 4 & -35 \\
4 & 261 & 122925 & 524 & 4 & -36 \\
4 & 261 & 122935 & 526 & 4 & -37 \\
4 & 261 & 122946 & 528 & 4 & -38 \\
4 & 261 & 122957 & 530 & 4 & -39 \\
4 & 261 & 123007 & 532 & 4 & -40 \\
4 & 261 & 123017 & 534 & 4 & -41 \\
4 & 261 & 123028 & 536 & 4 & -42 \\
4 & 261 & 123039 & 538 & 4 & -43 \\
4 & 261 & 123050 & 540 & 4 & -44 \\
4 & 261 & 123101 & 542 & 4 & -45 \\
4 & 261 & 123110 & 544 & 4 & -46 \\
4 & 261 & 123121 & 546 & 4 & -47 \\
4 & 261 & 123132 & 548 & 4 & -48 \\
4 & 261 & 123142 & 550 & 4 & -49 \\
4 & 261 & 123155 & 552 & 4 & -50 \\
4 & 261 & 123206 & 554 & 4 & -51 \\
4 & 261 & 123216 & 556 & 4 & -52 \\
5 & 261 & 123617 & 568 & 5 & 0 \\
5 & 261 & 123629 & 570 & 5 & -1 \\
5 & 261 & 123641 & 572 & 5 & -2 \\
5 & 261 & 123652 & 574 & 5 & -3 \\
5 & 261 & 123703 & 576 & 5 & -4 \\
5 & 261 & 123719 & 578 & 5 & -5 \\
5 & 261 & 123731 & 580 & 5 & -6 \\
5 & 261 & 123742 & 582 & 5 & -7 \\
5 & 261 & 123755 & 584 & 5 & -8 \\
5 & 261 & 123809 & 586 & 5 & -9 \\
5 & 261 & 123818 & 588 & 5 & -10 \\
5 & 261 & 123829 & 590 & 5 & -11 \\
5 & 261 & 123839 & 592 & 5 & -12 \\
5 & 261 & 123849 & 594 & 5 & -13 \\
5 & 261 & 123901 & 596 & 5 & -14 \\
5 & 261 & 123911 & 598 & 5 & -15 \\
5 & 261 & 123922 & 600 & 5 & -16 \\
5 & 261 & 123933 & 602 & 5 & -17 \\
5 & 261 & 123944 & 604 & 5 & -18 \\
5 & 261 & 123956 & 606 & 5 & -19 \\
5 & 261 & 124007 & 608 & 5 & -20 \\
5 & 261 & 124019 & 610 & 5 & -21 \\
5 & 261 & 124030 & 612 & 5 & -22 \\
5 & 261 & 124040 & 614 & 5 & -23 \\
& 261 & 124052 & 616 & 5 & -24 \\
5 & 261 & 124104 & 618 & 5 & -25 \\
5 & 124217 & 620 & 5 & -26 \\
5 & & \\
5 & 5237 & 624 & 5 & -27 \\
5 & & & &
\end{tabular}
uncorr. corr. dif. UncOrr. dif. uncorr. corr. $\begin{array}{lllll}51369.5 & 2.2 & 51363.4 & 51358.0 & 5.4\end{array}$ $\begin{array}{llllll}51362.5 & 51360.3 & 2.2 & 51353.4 & 51348.0 & 5.4\end{array}$ $\begin{array}{llllll}51353.6 & 51351.3 & 2.3 & 51340.0 & 51334.5 & 5.5\end{array}$ $\begin{array}{llllll}51343.8 & 51341.5 & 2.3 & 51326.4 & 51320.9 & 5.5\end{array}$ $\begin{array}{llllll}51328.7 & 51326.4 & 2.3 & 51317.4 & 51311.8 & 5.6\end{array}$ $\begin{array}{llllll}51321.3 & 51319.0 & 2.3 & 51305.8 & 51300.1 & 5.7\end{array}$ $\begin{array}{llllll}51305.5 & 51303.2 & 2.3 & 51288.1 & 51282.4 & 5.7\end{array}$ $\begin{array}{llllll}51284.8 & 51282.4 & 2.4 & 51266.3 & 51260.5 & 5.8\end{array}$ $\begin{array}{llllll}51262.8 & 51260.4 & 2.4 & 51248.1 & 51242.2 & 5\end{array}$ $\begin{array}{llllll}51236.4 & 51234.0 & 2.4 & 51222.5 & 51216.6 & 5\end{array}$

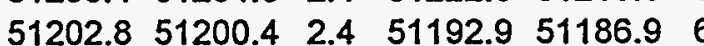
$\begin{array}{llllll}51169.9 & 51167.5 & 2.4 & 51159.7 & 51153.6 & 6.1\end{array}$ $\begin{array}{lllll}51118.8 & 51116.3 & 2.5 & 51118.6 & 51112.5\end{array}$ $\begin{array}{llllll}51050.7 & 51048.2 & 2.5 & 51058.5 & 51052.3 & 6\end{array}$ $\begin{array}{lllllll}50956.6 & 50954.1 & 2.5 & 50982.6 & 50976.4 & 6.2\end{array}$ $\begin{array}{llllll}50847.5 & 50845.0 & 2.5 & 50883.3 & 50877.0 & 6.3\end{array}$ $\begin{array}{llllll}50768.2 & 50765.7 & 2.5 & 50767.6 & 50761.2 & 6.4\end{array}$ $\begin{array}{llllll}50714.6 & 50712.0 & 2.6 & 50644.2 & 50637.8 & 6.4\end{array}$ $\begin{array}{lllll}51131.0 & 51128.4 & 2.6 & 50719.8 & 50713.3\end{array}$ $\begin{array}{llllll}52276.3 & 52273.7 & 2.6 & 52076.4 & 52069.8\end{array}$

$\begin{array}{llllll}50014.6 & 50011.6 & 3.0 & 58577.9 & 58571.2\end{array}$

$50527.4 \quad 50524.4 \quad 3.0 \quad 50260.6 \quad 50253.9$

$\begin{array}{llllll}50964.9 & 50961.9 & 3.0 & 50862.7 & 5008 & 56.0\end{array}$

$\begin{array}{llllll}51165.1 & 51162.0 & 3.1 & 51102.8 & 51096.1\end{array}$

$\begin{array}{llllll}51256.4 & 51253.3 & 3.1 & 51217.1 & 51210.4\end{array}$

$\begin{array}{llllll}51311.8 & 51308.7 & 3.1 & 51277.5 & 51270.9 & 6.6\end{array}$

$\begin{array}{lllllll}51331.1 & 51327.9 & 3.2 & 51308.0 & 51301.4 & 6.6\end{array}$

$\begin{array}{llllll}51343.1 & 51339.9 & 3.2 & 51322.3 & 513115.7 & 6.6\end{array}$

$\begin{array}{llllll}51327.7 & 51324.5 & 3.2 & 51327.0 & 51320.4 & 6.6\end{array}$

$\begin{array}{lllllll}51304.0 & 51300.8 & 3.2 & 51296.1 & 51289.6 & 6.5\end{array}$

$\begin{array}{llllll}51263.5 & 51260.2 & 3.3 & 51235.5 & 51229.0 & 6.5\end{array}$

$\begin{array}{llllll}51223.6 & 51220.3 & 3.3 & 51178.3 & 51171.8 & 6.5\end{array}$ $\begin{array}{lllllll}51207.7 & 51204.4 & 3.3 & 51140.7 & 51134.2 & 6.5\end{array}$ $\begin{array}{llllll}51239.4 & 51236.1 & 3.3 & 51.158 .0 & 51151.5 & 6.5\end{array}$ $\begin{array}{lllllll}51293.0 & 51289.6 & 3.4 & 51226.3 & 51219.8 & 6.5\end{array}$ $\begin{array}{lllllll}51326.5 & 51323.1 & 3.4 & 51291.0 & 51284.6 & 6.4\end{array}$ $\begin{array}{llllll}51345.9 & 51342.5 & 3.4 & 51329.9 & 51323.5 & 6.4\end{array}$ $\begin{array}{llllll}51368.5 & 51365.1 & 3.4 & 51340.0 & 51333.6 & 6.4\end{array}$ $\begin{array}{lllllll}51384.3 & 51380.8 & 3.5 & 51386.7 & 51380.3 & 6.4\end{array}$ $\begin{array}{lllllll}51392.5 & 51389.0 & 3.5 & 51401.4 & 51395.0 & 6.4\end{array}$ $\begin{array}{lllllll}51391.4 & 51387.9 & 3.5 & 51382.7 & 51376.4 & 6.3\end{array}$ $\begin{array}{llllll}51388.0 & 51384.5 & 3.5 & 51392.8 & 51386.5 & 6.3\end{array}$ $\begin{array}{lllllll}51386.3 & 51382.7 & 3.6 & 51373.3 & 51367.0 & 6.3\end{array}$ $\begin{array}{lllllll}51385.9 & 51382.3 & 3.6 & 51381.8 & 51375.5 & 6.3\end{array}$ $\begin{array}{lllllll}51389.6 & 51386.0 & 3.6 & 51388.4 & 51382.1 & 6.3\end{array}$ $\begin{array}{lllllll}51388.4 & 51384.8 & 3.6 & 51389.2 & 51382.9 & 6.3\end{array}$ $\begin{array}{lllllll}51375.8 & 51372.0 & 3.8 & 51380.8 & 51374.7 & 6.1\end{array}$ $\begin{array}{lllllll}51376.4 & 51372.6 & 3.8 & 51376.1 & 51370.0 & 6.1\end{array}$ $\begin{array}{lllllll}51375.3 & 51371.5 & 3.8 & 51374.8 & 51368.7 & 6.1\end{array}$
VERT. dif. GRAD.(nT/m) $-13.6$ $-14.9$ $-22.3$ $-28.5$ $-18.5$ $-25.4$ $-28.5$ $-30.3$ $-24.1$ $-22.8$ $-16.2$ $-16.7$ $-0.3$ 12.8 42.6 58.7 $-1.0$ $-115.4$ $-674.1$ $-327.7$ 14038.2 $-437.4$

$-167.5$

$-102.1$

$-64.4$

$-56.2$

$-37.9$

$-34.1$ $-1.1$

$-12.9$

$-45.9$

$-74.3$

$-109.8$

$-133.4$

$-109.3$

$-58.2$

$-26.2$

$-46.7$

3.9

14.6

$-14.3$

7.9

$-21.3$

\section{$-6.7$}

$-2.0$

1.3

8.2

$-0.5$ $-0.8$ 
Hydroflouric Acid Spill Magnetic Survey Data

TOP BOTTOM VERT.

LINE DAY TIME STA. $X \quad Y$ uncorr. corr. dif. uncorr. corr. dif. GRAD.(nT/m)

$\begin{array}{llllll}5 & 261 & 124248 & 626 & 5 & -29 \\ 5 & 261 & 124259 & 628 & 5 & -30 \\ 5 & 261 & 124310 & 630 & 5 & -31 \\ 5 & 261 & 124320 & 632 & 5 & -32 \\ 5 & 261 & 124330 & 634 & 5 & -33 \\ 5 & 261 & 124340 & 636 & 5 & -34 \\ 5 & 261 & 124351 & 638 & 5 & -35 \\ 5 & 261 & 124401 & 640 & 5 & -36 \\ 5 & 261 & 124411 & 642 & 5 & -37 \\ 5 & 261 & 124422 & 644 & 5 & -38 \\ 5 & 261 & 124432 & 646 & 5 & -39 \\ 5 & 261 & 124443 & 648 & 5 & -40 \\ 5 & 261 & 124453 & 650 & 5 & -41 \\ 5 & 261 & 124504 & 652 & 5 & -42 \\ 5 & 261 & 124513 & 654 & 5 & -43 \\ 5 & 261 & 124523 & 656 & 5 & -44 \\ 5 & 261 & 124533 & 658 & 5 & -45 \\ 5 & 261 & 124544 & 660 & 5 & -46 \\ 5 & 261 & 124554 & 662 & 5 & -47 \\ 5 & 261 & 124605 & 664 & 5 & -48 \\ 5 & 261 & 124616 & 666 & 5 & -49 \\ 5 & 261 & 124626 & 668 & 5 & -50 \\ 5 & 261 & 124636 & 670 & 5 & -51 \\ 6 & 261 & 125447 & 682 & 6 & 0 \\ 6 & 261 & 125459 & 684 & 6 & -1 \\ 6 & 261 & 125510 & 686 & 6 & -2 \\ 6 & 261 & 125520 & 688 & 6 & -3 \\ 6 & 261 & 125529 & 690 & 6 & -4 \\ 6 & 261 & 125540 & 692 & 6 & -5 \\ 6 & 261 & 125553 & 694 & 6 & -6 \\ 6 & 261 & 125603 & 696 & 6 & -7 \\ 6 & 261 & 125614 & 698 & 6 & -8 \\ 6 & 261 & 125625 & 700 & 6 & -9 \\ 6 & 261 & 125635 & 702 & 6 & -10 \\ 6 & 261 & 125646 & 704 & 6 & -11 \\ 6 & 261 & 125657 & 706 & 6 & -12 \\ 6 & 261 & 125707 & 708 & 6 & -13 \\ 6 & 261 & 125717 & 710 & 6 & -14 \\ 6 & 261 & 125727 & 712 & 6 & -15 \\ 6 & 261 & 125738 & 714 & 6 & -16 \\ 6 & 261 & 125748 & 716 & 6 & -17 \\ 6 & 261 & 125759 & 718 & 6 & -18 \\ 6 & 261 & 125808 & 720 & 6 & -19 \\ 6 & 261 & 125819 & 722 & 6 & -20 \\ 6 & 261 & 125830 & 724 & 6 & -21 \\ 6 & 261 & 125840 & 726 & 6 & -22 \\ 6 & 261 & 125849 & 728 & 6 & -23 \\ 6 & 261 & 125900 & 730 & 6 & -24 \\ 6 & 261 & 125911 & 732 & 6 & -25 \\ & & & & & \end{array}$

$\begin{array}{lllll}51372.8 & 51368.9 & 3.9 & 51378.8 & 51372.7\end{array}$

$\begin{array}{llllll}51372.1 & 51368.2 & 3.9 & 51379.7 & 51373.6\end{array}$

$\begin{array}{llllll}51366.0 & 51362.1 & 3.9 & 51373.5 & 51367.5\end{array}$

$\begin{array}{lllll}51360.2 & 51356.3 & 3.9 & 51366.5 & 51360.5\end{array}$

$\begin{array}{llllll}51354.4 & 51350.4 & 4.0 & 51357.0 & 51351.0 & 6.0\end{array}$

$\begin{array}{lllllll}51347.4 & 51343.4 & 4.0 & 51352.5 & 51346.5 & 6.0\end{array}$

$\begin{array}{lllllll}51336.9 & 51332.9 & 4.0 & 51337.6 & 51331.6 & 6.0\end{array}$

$\begin{array}{lllllll}51323.3 & 51319.3 & 4.0 & 51321.9 & 51315.9 & 6.0\end{array}$

$\begin{array}{lllllll}51308.7 & 51304.6 & 4.1 & 51312.4 & 51306.5 & 5.9\end{array}$

$\begin{array}{lllllll}51291.7 & 51287.6 & 4.1 & 51292.6 & 51286.7 & 5.9\end{array}$

$\begin{array}{lllllll}51270.2 & 51266.1 & 4.1 & 51273.1 & 51267.2 & 5.9\end{array}$

$\begin{array}{lllllll}51241.3 & 51237.2 & 4.1 & 51251.3 & 51245.4 & 5.9\end{array}$

$\begin{array}{lllllll}51211.7 & 51207.6 & 4.1 & 51217.4 & 51211.5 & 5.9\end{array}$

$\begin{array}{lllllll}51179.5 & 51175.3 & 4.2 & 51184.1 & 51178.2 & 5.9\end{array}$

$\begin{array}{llllll}51134.9 & 51130.7 & 4.2 & 51137.8 & 51132.0 & 5.8\end{array}$

$\begin{array}{lllllll}51061.7 & 51057.5 & 4.2 & 51074.1 & 51068.3 & 5.8\end{array}$

$\begin{array}{lllllll}50972.5 & 50968.3 & 4.2 & 51010.6 & 51004.8 & 5.8\end{array}$

$\begin{array}{lllllll}50876.0 & 50871.7 & 4.3 & 50943.2 & 50937.4 & 5.8\end{array}$

$\begin{array}{llllllll}50695.3 & 50691.0 & 4.3 & 50812.5 & 50806.7 & 5.8\end{array}$

$\begin{array}{llllllll}50249.6 & 50245.3 & 4.3 & 50497.1 & 50491.3 & 5.8\end{array}$

$\begin{array}{llllll}49768.2 & 49763.9 & 4.3 & 50102.9 & 50097.2 & 5.7\end{array}$

$\begin{array}{lllllll}48922.8 & 48918.4 & 4.4 & 49311.7 & 49306.0 & 5.7\end{array}$

$\begin{array}{llllll}52231.9 & 52227.5 & 4.4 & 58525.6 & 58519.9 & 5.7\end{array}$

$\begin{array}{lllllll}49940.6 & 49933.8 & 6.8 & 58589.4 & 58585.5 & 3.9\end{array}$

$\begin{array}{lllllll}50338.8 & 50331.9 & 6.9 & 49790.2 & 49786.2 & 4.0\end{array}$

$\begin{array}{lllllll}50887.8 & 50880.9 & 6.9 & 50710.2 & 50706.1 & 4.1\end{array}$

$\begin{array}{llllll}51130.7 & 51123.8 & 6.9 & 51047.9 & 51043.8 & 4.1\end{array}$

$\begin{array}{lllllll}51245.4 & 51238.4 & 7.0 & 51185.7 & 51181.5 & 4.2\end{array}$

$\begin{array}{llllll}51305.7 & 51298.7 & 7.0 & 51259.4 & 51255.1 & 4.3\end{array}$

$\begin{array}{lllllll}51336.2 & 51329.1 & 7.1 & 51298.2 & 51293.8 & 4.4\end{array}$

$\begin{array}{lllllll}51345.1 & 51338.0 & 7.1 & 51321.4 & 51317.0 & 4.4\end{array}$

$\begin{array}{lllllll}51350.5 & 51343.4 & 7.1 & 51326.9 & 51322.4 & 4.5\end{array}$

$\begin{array}{lllllll}51339.8 & 51332.6 & 7.2 & 51314.8 & 51310.2 & 4.6\end{array}$

$\begin{array}{lllllll}51326.3 & 51319.1 & 7.2 & 51283.7 & 51279.1 & 4.6\end{array}$

$\begin{array}{lllllll}51314.8 & 51307.6 & 7.2 & 51261.7 & 51257.0 & 4.7\end{array}$

$\begin{array}{lllllll}51310.3 & 51303.0 & 7.3 & 51253.6 & 51248.8 & 4.8\end{array}$

$\begin{array}{lllllll}51317.8 & 51310.5 & 7.3 & 51254.6 & 51249.8 & 4.8\end{array}$

$\begin{array}{llllllll}51334.6 & 51327.3 & 7.3 & 512668.2 & 51263.3 & 4.9\end{array}$

$\begin{array}{lllllll}51356.8 & 51349.4 & 7.4 & 51292.9 & 51287.9 & 5.0\end{array}$

$\begin{array}{lllllll}51375.7 & 51368.3 & 7.4 & 51334.0 & 51329.0 & 5.0\end{array}$

$\begin{array}{lllllll}51390.0 & 51382.6 & 7.4 & 51311.8 & 51366.7 & 5.1\end{array}$

$\begin{array}{lllllll}51405.6 & 51398.1 & 7.5 & 513386.2 & 51381.0 & 5.2\end{array}$

$\begin{array}{lllllll}51410.9 & 51403.4 & 7.5 & 51388.1 & 51382.9 & 5.2\end{array}$

$\begin{array}{llllll}51416.3 & 51408.8 & 7.5 & 51415.4 & 51410.1 & 5.3\end{array}$

$\begin{array}{lllllll}51407.7 & 51400.1 & 7.6 & 51370.5 & 51365.1 & 5.4\end{array}$

$\begin{array}{llllll}51394.0 & 51386.4 & 7.6 & 51333.8 & 51328.4 & 5.4\end{array}$

$\begin{array}{llllll}51388.5 & 51380.9 & 7.6 & 513151.0 & 51345.5 & 5.5\end{array}$

$\begin{array}{llllll}51387.0 & 51379.3 & 7.7 & 51359.5 & 51354.0 & 5.5\end{array}$

$\begin{array}{llllll}51388.0 & 51380.3 & 7.7 & 51369.8 & 51364.2 & 5.6\end{array}$
9.8

12.5

12.3

10.3

4.3

8.4

1.1

$-2.3$

6.1

1.5

4.8

16.4

9.3

7.5

4.8

20.3

62.5

110.2

192.1

405.7

548.7

637.5

10317.5

14178.4

$-899.3$

$-291.2$

$-135.7$

$-97.9$

$-75.9$

$-62.3$

$-38.9$

$-38.7$

$-41.0$

$-69.8$

$-87.1$

$-93.0$

$-103.6$

$-108.9$

$-104.8$

$-68.4$

$-29.8$

$-31.8$

$-37.4$

$-1.5$

$-61.0$

$-98.7$

$-61.5$

$-45.1$

$-29.8$ 
TOP BOTTOM VERT.

LINE DAY TIME STA. $X \quad Y$ uncorr. corr. dif. uncorr. corr. dif. GRAD.(nT/m)

\begin{tabular}{|c|c|c|c|c|c|c|c|c|c|c|c|c|}
\hline 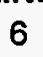 & 261 & 130024 & 734 & 6 & -26 & 51390.1 & 51382.2 & 7.9 & 51366.3 & 51360.2 & 6.1 & -39.0 \\
\hline & 261 & 130034 & 736 & 6 & -27 & 51379.8 & 51371.8 & 8.0 & 51364.8 & 51358.7 & 6.1 & -24.6 \\
\hline & 261 & 130043 & 738 & 6 & -28 & 51384.4 & 51376.4 & 8.0 & 51361.1 & 51354.9 & 6.2 & -38.2 \\
\hline & 261 & 130054 & 740 & 6 & -29 & 51379.7 & 51371.7 & 8.0 & 51358.1 & 51351.8 & 6.3 & -35.4 \\
\hline & 261 & 130104 & 742 & 6 & -30 & 51377.5 & 51369.4 & 8.1 & 51359.2 & 51352.9 & 6.3 & -30.0 \\
\hline & 261 & 130113 & 744 & 6 & -31 & 51371.2 & 51363.1 & 8.1 & 51349.1 & 51342.7 & 6.4 & -36.2 \\
\hline & 261 & 130124 & 746 & 6 & -32 & 51323.6 & 51315.5 & 8.1 & 51340.6 & $5: 1334.1$ & 6.5 & 27.9 \\
\hline & 261 & 130135 & 748 & 6 & -33 & 1352.8 & 51344.6 & 8.2 & 51331.2 & 51324.7 & 6.5 & -35.4 \\
\hline 6 & 261 & 130146 & 750 & 6 & -34 & 1339.2 & 51331.0 & 8.2 & 323.0 & 51316.4 & 6.6 & -26.6 \\
\hline 6 & 261 & 130157 & 752 & 6 & -35 & 1321.2 & 51313.0 & 8.2 & 306.9 & 51300.2 & 6.7 & -23.4 \\
\hline 6 & 261 & 130207 & 754 & 6 & -36 & 94.6 & 51296.3 & 8.3 & 51290.4 & 51283.7 & 6.7 & -23.3 \\
\hline 6 & 261 & 130219 & 756 & 6 & -37 & 51292.1 & 51283.8 & 8.3 & 51271.2 & 51264.4 & 6.8 & -34.3 \\
\hline 6 & 261 & 130229 & 758 & 6 & -38 & 51268.3 & 51260.0 & 8.3 & 51251.7 & 51244.8 & 6.9 & -27.2 \\
\hline 6 & 261 & 130239 & 760 & 6 & -39 & 41.8 & 51233.4 & 8.4 & 51226.9 & 51220.0 & 6.9 & -24.4 \\
\hline 6 & 261 & 130249 & 762 & 6 & -40 & 06.9 & 51198.5 & 8.4 & 189.5 & 182.5 & 7.0 & -28.5 \\
\hline 6 & 261 & 130300 & 764 & 6 & -41 & 55.7 & 51147.3 & 8.4 & 41.0 & 33.9 & 7.1 & -24.1 \\
\hline 6 & 261 & 130310 & 766 & 6 & -42 & 94.8 & 51086.3 & 8.5 & 88.2 & 81.1 & 7.1 & -10.8 \\
\hline 6 & 261 & 130320 & 768 & 6 & -43 & 10.8 & 51002.3 & 8.5 & 13.8 & 006.6 & 7.2 & 4.9 \\
\hline 6 & 261 & 130330 & 770 & 6 & -44 & 50909.2 & 50900.7 & 8.5 & 50934.5 & 50927.2 & 7.3 & 41.5 \\
\hline 6 & 261 & 130341 & 772 & 6 & -45 & 50740.4 & 50731.8 & 8.6 & 50818.8. & 50811.5 & 7.3 & 128.5 \\
\hline 6 & 261 & 130352 & 774 & 6 & -46 & 50445.2 & 50436.6 & 8.6 & $50653.0^{\circ}$ & 50645.6 & 7.4 & 340.7 \\
\hline 6 & 261 & 130404 & 776 & 6 & -47 & 49825.3 & 49816.7 & 8.6 & 505060.4 & 50498.9 & 7.5 & 1116.6 \\
\hline 6 & 261 & 130415 & 778 & 6 & -48 & 58595.0 & 58586.3 & 8.7 & & & 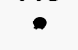 & * \\
\hline 7 & 261 & 130618 & 789 & 7 & 0 & 58.8 & 48750.9 & 7.9 & 553.4 & 58547.0 & 6.4 & 16056.7 \\
\hline 7 & 261 & 130629 & 791 & 7 & -1 & 141.8 & 52033.8 & 8.0 & 49404.5 & 49398.1 & 6.4 & -4323.4 \\
\hline 7 & 261 & 130642 & 793 & 7 & -2 & 25.2 & 17.1 & 8.1 & 34.8 & 50628.4 & 6.4 & -312.1 \\
\hline 7 & 261 & 130652 & 795 & 7 & -3 & 04.5 & 51096.4 & 8.1 & 51036.9 & 51030.5 & 6.4 & -110.8 \\
\hline 7 & 261 & 130703 & 797 & 7 & -4 & 3.3 & 15.1 & 8.2 & 39.3 & 51182.9 & 6.4 & -55.7 \\
\hline 7 & 261 & 130713 & 799 & 7 & -5 & 93.4 & 51285.2 & 8.2 & 9.5 & 63.1 & 6.4 & -39.2 \\
\hline 7 & 261 & 130723 & 801 & 7 & -6 & 51322.0 & 51313.8 & 8.2 & 51309.2 & 51302.8 & 6.4 & -21.0 \\
\hline 7 & 261 & 130734 & 803 & 7 & -7 & 51335.1 & 51326.8 & 8.3 & 51327.0̣ & 51320.6 & 6.4 & -13.3 \\
\hline 7 & 261 & 130744 & 805 & 7 & -8 & 51337.2 & 51328.9 & 8.3 & 51327.4 & 51321.1 & 6.3 & -16.1 \\
\hline 7 & 261 & 130754 & 807 & 7 & -9 & 51332.2 & 51323.8 & 8.4 & 51308.1 & 51301.8 & 6.3 & -39.5 \\
\hline 7 & 261 & 130804 & 809 & 7 & -10 & 32.2 & 51323.8 & 8.4 & 51297.9 & 51291.6 & 6.3 & -56.2 \\
\hline 7 & 261 & 130814 & 811 & 7 & -11 & 30.8 & 51322.3 & 8.5 & 3.9 & 51287.6 & 6.3 & -60.5 \\
\hline 7 & 261 & 130825 & 813 & 7 & -12 & & 321.0 & 8.5 & 13.0 & 51296.7 & 6.3 & -43.4 \\
\hline 7 & 261 & 130835 & 815 & 7 & -13 & 36.7 & 28.1 & 8.6 & & 310.6 & 6.3 & -32.5 \\
\hline 7 & 261 & 130845 & 817 & 7 & -14 & 51345.5 & 51336.9 & 8.6 & 51330.4 & 1324.1 & 6.3 & -24.8 \\
\hline 7 & 261 & 130856 & 819 & 7 & -15 & 51361.2 & 51352.5 & 8.7 & 51342.1 & 51335.8 & 6.3 & -31.3 \\
\hline 7 & 261 & 130906 & 821 & 7 & -16 & 51375.8 & 51367.1 & 8.7 & 51376.5 & 51370.2 & 6.3 & 1.1 \\
\hline 7 & 261 & 130917 & 823 & 7 & -17 & 51389.1 & 51380.3 & 8.8 & 51377.7 & 51371.5 & 6.2 & -18.7 \\
\hline 7 & 261 & 130928 & 825 & 7 & -18 & 51393.7 & 51384.9 & 8.8 & 513130.5 & 51374.3 & 6.2 & -21.6 \\
\hline 7 & 261 & 130940 & 827 & 7 & -19 & 395.4 & 51386.5 & 8.9 & $51,406.2$ & 51400.0 & 6.2 & 17.7 \\
\hline 7 & 261 & 130950 & 829 & 7 & -20 & 51395.3 & 51386.4 & 8.9 & 51412.8 & 51406.6 & 6.2 & 28.7 \\
\hline 7 & 261 & 131001 & 831 & 7 & -21 & 51394.2 & 51385.2 & 9.0 & 51389.5 & 51383.3 & 6.2 & -7.7 \\
\hline 7 & 261 & 131012 & 833 & 7 & -22 & 51386.4 & 51377.4 & 9.0 & 51393.9 & 51387.7 & 6.2 & 12.3 \\
\hline 7 & 261 & 131023 & 835 & 7 & -23 & 51370.1 & 51361.0 & 9.1 & 51349.8 & 5 & 6.2 & -33.3 \\
\hline 7 & 261 & 131034 & 837 & 7 & -24 & 51363.9 & 51354.8 & 9.1 & 51352.5 & 513 & 6.2 & -18.7 \\
\hline & 261 & 131045 & 839 & 7 & -25 & 51367.5 & 51358.3 & 9.2 & 51357.5 & 51351.4 & 6.1 & -16.4 \\
\hline
\end{tabular}


TOP

LINE DAY TIME STA. $X \quad Y$

7

7

7

7

7

$$
\begin{array}{lllll}
261 & 131226 & 841 & 7 & -26
\end{array}
$$

-41
-42

$-43$

$-44$

$-45$

$-46$

$-47$

$-48$

$-49$

$-50$

0

$-1$

$-2$

$-3$

$-4$

$-5$

$-6$

$-7$

$-8$

$-9$

$-10$

$-11$

$-12$

$-13$

$-14$

$-15$

$-16$

$-17$

$-18$

$-20$

$-21$

$-22$ $-23$
$-19$ uncorr corr.

dif. uncorr. corr.

$\begin{array}{lllllll}51376.2 & 51366.5 & 9.7 & 51360.4 & 51354.4 & 6.0\end{array}$

$\begin{array}{lllllll}51380.4 & 51370.7 & 9.7 & 51363.9 & 51357.9 & 6.0\end{array}$

$\begin{array}{llllll}51371.7 & 51361.9 & 9.8 & 51363.3 & 51357.3 & 6.0\end{array}$

$\begin{array}{lllllll}51363.8 & 51354.0 & 9.8 & 51362.2 & 51356.2 & 6.0\end{array}$

$\begin{array}{lllllll}51360.7 & 51350.8 & 9.9 & 51364.6 & 51358.6 & 6.0\end{array}$

$\begin{array}{lllllll}51352.6 & 51342.7 & 9.9 & 51351.0 & 51345.0 & 6.0\end{array}$

$\begin{array}{lllllll}51345.9 & 51335.9 & 10.0 & 51344.1 & 51338.1 & 6.0\end{array}$

$\begin{array}{llllll}51334.0 & 51324.0 & 10.0 & 51331.4 & 51325.5 & 5.9\end{array}$

$\begin{array}{llllll}51324.9 & 51314.8 & 10.1 & 51322.2 & 51316.3 & 5.9\end{array}$

$\begin{array}{llllll}51309.7 & 51299.6 & 10.1 & 51308.7 & 51302.8 & 5.9\end{array}$

$\begin{array}{llllll}51290.3 & 51280.2 & 10.1 & 51289.8 & 51283.9 & 5.9\end{array}$

$\begin{array}{llllll}51277.6 & 51267.4 & 10.2 & 51276.5 & 51270.6 & 5.9\end{array}$

$51257.4 \quad 51247.2 \quad 10.2 \quad 51260.4 \quad 51254.5 \quad 5.9$

$\begin{array}{llllll}51230.6 & 51220.3 & 10.3 & 51230.4 & 51224.5 & 5.9\end{array}$

$\begin{array}{llllll}51190.7 & 51180.4 & 10.3 & 51196.1 & 51190.2 & 5.9\end{array}$

$\begin{array}{llllll}51138.9 & 51128.5 & 10.4 & 51143.4 & 51137.5 & 5.9\end{array}$

$\begin{array}{llllll}51068.8 & 51058.4 & 10.4 & 51080.3 & 51074.5 & 5.8\end{array}$

$\begin{array}{llllll}50991.6 & 50981.1 & 10.5 & 51013.2 & 51007.4 & 5.8\end{array}$

$\begin{array}{llllll}50885.7 & 50875.2 & 10.5 & 50938.1 & 50932.3 & 5.8\end{array}$

$\begin{array}{lllllll}50717.4 & 50706.8 & 10.6 & 50834.8 & 50829.0 & 5.8\end{array}$

$\begin{array}{llllll}50435.8 & 50425.2 & 10.6 & 50670.2 & 50664.4 & 5.8\end{array}$

$\begin{array}{llllll}49864.7 & 49854.0 & 10.7 & 50622.3 & 50616.5 & 5.8\end{array}$

$\begin{array}{llllll}52138.3 & 52127.6 & 10.7 & 50032.5 & 500 \dot{2} 26.7 & 5.8\end{array}$

$\begin{array}{llllll}52031.4 & 52020.6 & 10.8 & 58572.9 & 58567.1 & 5.8\end{array}$

$\begin{array}{llllll}51982.2 & 51971.4 & 10.8 & 58628.4 & 58622.7 & 5.7\end{array}$

$\begin{array}{llllll}58586.8 & 58576.0 & 10.8 & 58528.1 & 58522.4 & 5.7\end{array}$

$\begin{array}{llllll}52180.2 & 52169.4 & 10.8 & 49017.3 & 49011.5 & 5.8\end{array}$

$\begin{array}{llllll}50736.9 & 50726.1 & 10.8 & 50563.0 & 50557.1 & 5.9\end{array}$

$\begin{array}{llllll}51065.2 & 51054.4 & 10.8 & 509 \dot{9} 2.6 & 50 \dot{9} 86.6 & 6.0\end{array}$

$\begin{array}{llllll}51211.8 & 51201.0 & 10.8 & 51174.3 & 51168.3 & 6.0\end{array}$

$\begin{array}{lllllll}51273.9 & 51263.1 & 10.8 & 51253.9 & 51247.8 & 6.1\end{array}$

$\begin{array}{llllll}51309.8 & 51299.0 & 10.8 & 51294.6 & 51288.4 & 6.2\end{array}$

$\begin{array}{lllllll}51324.7 & 51313.9 & 10.8 & 51305.1 & 51298.8 & 6.3\end{array}$

$\begin{array}{llllll}51327.6 & 51316.8 & 10.8 & 51299.2 & 51292.8 & 6.4\end{array}$

$\begin{array}{llllll}51333.4 & 51322.6 & 10.8 & 51297.5 & 51291.0 & 6.5\end{array}$

$\begin{array}{llllll}51339.8 & 51329.0 & 10.8 & 51312.9 & 51306.4 & 6.5\end{array}$

$\begin{array}{llllll}51349.1 & 51338.3 & 10.8 & 51342.2 & 51335.6 & 6.6\end{array}$

$\begin{array}{llllll}51353.6 & 51342.8 & 10.8 & 51337.1 & 51330.4 & 6.7\end{array}$

$\begin{array}{lllllll}51363.5 & 51352.7 & 10.8 & 51350.7 & 51343.9 & 6.8\end{array}$

$\begin{array}{llllll}51370.3 & 51359.5 & 10.8 & 51364.6 & 51357.8 & 6.8\end{array}$

$\begin{array}{llllll}51374.4 & 51363.6 & 10.8 & 51348.1 & 51341.2 & 6.9\end{array}$

$\begin{array}{llllll}51381.5 & 51370.7 & 10.8 & 51357.6 & 51350.6 & 7.0\end{array}$

$\begin{array}{lllllll}51387.3 & 51376.5 & 10.8 & 51405.7 & 51398.6 & 7.1\end{array}$

$\begin{array}{llllll}51387.9 & 51377.1 & 10.8 & 51402.4 & 51395.2 & 7.2\end{array}$

$\begin{array}{lllllll}51378.3 & 51367.5 & 10.8 & 513775.5 & 51368.2 & 7.3\end{array}$

$\begin{array}{llllll}51374.4 & 51363.6 & 10.8 & 51342.6 & 51335.2 & 7.4\end{array}$

$\begin{array}{lllllll}51383.9 & 51373.1 & 10.8 & 513 ̈ 88.1 & 51380.7 & 7.4\end{array}$

$\begin{array}{llllll}51385.1 & 51374.3 & 10.8 & 51408.4 & 51400.9 & 7.5\end{array}$

$\begin{array}{llllll}51358.3 & 51347.5 & 10.8 & 513 ̈ 35.1 & 51327.5 & 7.6\end{array}$
VERT.

RAD.(nT/m)

$-25.9$

$-27.0$

$-13.8$

$-2.6$

6.4

$-2.6$

$-3.0$

$-4.3$

$-4.4$

$-1.6$

$-0.8$

$-1.8$

4.9

$-0.3$

8.9

7.4

18.9

35.4

85.9

192.5

384.3

1242.0

$-3452.1$

10723.8

10895.4

$-96.2$

$-5185.1$

$-285.1$

$-119.0$

$-61.5$

$-32.8$

$-24.9$

$-32.1$

$-46.6$

$-58.9$

$-44.1$

$-11.3$

$-27.0$

$-21.0$

$-9.3$

$-43.1$

$-39.2$

30.2

23.8

$-4.6$

$-52.1$

6.9

38.2

$-38.0$ 
Hydroflouric Acid Spill Magnetic Survey Data

TOP

BOTTOM

LINE DAY TIME STA. $\mathrm{X}$

$$
\mathrm{Y}
$$
$-24$ $-25$ $-26$ $-27$ $-28$ $-29$ $-30$ $-31$ $-32$ $-33$ $-34$ $-35$ $-36$ $-37$ $-38$ $-39$ $-40$ $-41$ $-42$ $-43$ $-44$ $-45$ $-46$ $-47$ $-48$ $-49$ $\begin{array}{lll}261 & 132858 & 999\end{array}$ $\begin{array}{lll}261 & 133122 & 1011\end{array}$ $\begin{array}{lll}261 & 133134 & 1013\end{array}$ $\begin{array}{lll}261 & 133147 \quad 1015\end{array}$ $\begin{array}{lll}261 & 133201 & 1017\end{array}$ $\begin{array}{llll}261 & 133213 & 1019\end{array}$ $\begin{array}{lll}261 & 133225 & 1021\end{array}$ $\begin{array}{llll}261 & 133241 & 1023 & 9\end{array}$ $\begin{array}{llll}261 & 133251 & 1025 & 9\end{array}$ $\begin{array}{llll}261 & 133303 & 1027\end{array}$ $\begin{array}{llll}261 & 133313 & 1029 & 9\end{array}$ $\begin{array}{llll}261 & 133324 & 1031 & 9\end{array}$ $\begin{array}{llll}261 & 133335 & 1033 \quad 9\end{array}$ $\begin{array}{lll}261 & 133346 \quad 1035 \quad 9\end{array}$ $\begin{array}{llll}261 & 133358 & 1037 & 9\end{array}$ $\begin{array}{llll}261 & 133408 & 1039 & 9\end{array}$ $\begin{array}{llll}261 & 133418 & 1041 & 9\end{array}$ $\begin{array}{llll}261 & 133429 & 1043 & 9\end{array}$ $\begin{array}{llll}261 & 133441 & 1045 & 9\end{array}$ $\begin{array}{llll}261 & 133452 & 1047 & 9\end{array}$ $\begin{array}{llll}261 & 133503 & 1049 & 9\end{array}$ $\begin{array}{llll}261 & 133515 & 1051 & 9\end{array}$ $\begin{array}{llll}261 & 133526 & 1053 & 9\end{array}$ $\begin{array}{llll}261 & 133537 & 1055 & 9\end{array}$ uncorr. corr. dif. uncorr. corr. $\begin{array}{llllll}51333.6 & 51322.8 & 10.8 & 51281.2 & 51273.5 & 7.7\end{array}$ $\begin{array}{llllll}51339.3 & 51328.5 & 10.8 & 51328.4 & 51320.6 & 7.8\end{array}$ $\begin{array}{lllllll}51368.4 & 51357.6 & 10.8 & 51337.1 & 51328.7 & 8.4\end{array}$ $\begin{array}{lllllll}51376.2 & 51365.4 & 10.8 & 51353.2 & 51344.7 & 8.5\end{array}$ $\begin{array}{lllllll}51373.5 & 51362.7 & 10.8 & 51351.2 & 51342.6 & 8.6\end{array}$ $\begin{array}{lllllll}51369.0 & 51358.2 & 10.8 & 51346.8 & 51338.1 & 8.7\end{array}$ $\begin{array}{lllllll}51363.6 & 51352.8 & 10.8 & 51343.7 & 51334.9 & 8.8\end{array}$ $\begin{array}{llllllll}51358.6 & 51347.8 & 10.8 & 51334.7 & 51325.9 & 8.8\end{array}$ $\begin{array}{lllllll}51345.8 & 51335.0 & 10.8 & 51324.4 & 51315.5 & 8.9\end{array}$ $\begin{array}{lllllll}51332.9 & 51322.1 & 10.8 & 51311.1 & 51302.1 & 9.0\end{array}$ $\begin{array}{llllll}51318.4 & 51307.6 & 10.8 & 51295.3 & 51286.2 & 9.1\end{array}$ $\begin{array}{lllllll}51301.6 & 51290.8 & 10.8 & 51279.4 & 51270.2 & 9.2\end{array}$ $\begin{array}{lllllll}51289.3 & 51278.5 & 10.8 & 51265.4 & 51256.2 & 9.2\end{array}$ $\begin{array}{llllll}51268.4 & 51257.6 & 10.8 & 51245.3 & 51236.0 & 9.3\end{array}$ $\begin{array}{llllll}51247.9 & 51237.1 & 10.8 & 51227.5 & 51218.1 & 9.4\end{array}$ $\begin{array}{llllll}51207.5 & 51196.7 & 10.8 & 51190.6 & 51181.1 & 9.5\end{array}$ $\begin{array}{llllll}51163.8 & 51153.0 & 10.8 & 51147.6 & 51138.0 & 9.6\end{array}$ $\begin{array}{llllll}51102.7 & 51091.9 & 10.8 & 51094.3 & 51084.6 & 9.7\end{array}$ $\begin{array}{llllll}51013.7 & 51002.9 & 10.8 & 51013.6 & 51003.9 & 9.7\end{array}$ $\begin{array}{lllllll}50914.8 & 50904.0 & 10.8 & 50923.1 & 50913.3 & 9.8\end{array}$ $\begin{array}{lllllll}50758.0 & 50747.2 & 10.8 & 507.97 .3 & 50787.4 & 9.9\end{array}$ $\begin{array}{lllllll}50561.5 & 50550.7 & 10.8 & 50652.2 & 50642.2 & 10.0\end{array}$ $\begin{array}{lllllll}50175.1 & 50164.3 & 10.8 & 50544.4 & 50534.3 & 10.1\end{array}$ $\begin{array}{llllll}49406.9 & 49396.1 & 10.8 & 50730.5 & 50720.4 & 10.1\end{array}$ $\begin{array}{llllll}58606.2 & 58595.4 & 10.8 & 58568.4 & 58558.2 & 10.2\end{array}$ $\begin{array}{lllllll}58607.3 & 58596.5 & 10.8 & 58520.8 & 58010.5 & 10.3\end{array}$ $49014.6 \quad 49003.8 \quad 10.8$

$\begin{array}{lllllll}50126.3 & 50115.6 & 10.7 & 49552.9 & 49543.4 & 9.5\end{array}$ $\begin{array}{lllllll}50754.7 & 50744.0 & 10.7 & 50563.7 & 50554.2 & 9.5\end{array}$ $\begin{array}{llllll}51080.4 & 51069.7 & 10.7 & 50984.9 & 50975.5 & 9.4\end{array}$ $\begin{array}{lllllll}51216.4 & 51205.8 & 10.6 & 51156.6 & 51147.2 & 9.4\end{array}$ $\begin{array}{lllllll}51284.2 & 51273.6 & 10.6 & 51240.5 & 51231.1 & 9.4\end{array}$ $\begin{array}{lllllll}51320.1 & 51309.5 & 10.6 & 51277.9 & 51268.6 & 9.3\end{array}$ $\begin{array}{lllllll}51334.3 & 51323.7 & 10.6 & 51284.4 & 51275.1 & 9.3\end{array}$ $\begin{array}{lllllll}51341.6 & 51331.1 & 10.5 & 51297.6 & 51288.3 & 9.3\end{array}$ $\begin{array}{llllll}51349.9 & 51339.4 & 10.5 & 51305.5 & 51296.3 & 9.2\end{array}$ $\begin{array}{lllllll}51360.5 & 51350.0 & 10.5 & 51326.1 & 51316.9 & 9.2\end{array}$ $\begin{array}{lllllll}51371.6 & 51361.1 & 10.5 & 51343.7 & 51334.5 & 9.2\end{array}$ $\begin{array}{lllllll}51377.4 & 51367.0 & 10.4 & 51334.4 & 51325.3 & 9.1\end{array}$ $\begin{array}{llllll}51387.0 & 51376.6 & 10.4 & 51328.6 & 51319.5 & 9.1\end{array}$ $\begin{array}{llllll}51394.3 & 51383.9 & 10.4 & 51348.8 & 51339.7 & 9.1\end{array}$ $\begin{array}{lllllll}51397.0 & 51386.6 & 10.4 & 51357.9 & 51348.8 & 9.1\end{array}$ $\begin{array}{lllllll}51393.0 & 51382.7 & 10.3 & 51358.9 & 51349.9 & 9.0\end{array}$ $\begin{array}{lllllll}51394.2 & 51383.9 & 10.3 & 51380.3 & 51371.3 & 9.0\end{array}$ $\begin{array}{lllllll}51386.2 & 51375.9 & 10.3 & 51374.1 & 51365.1 & 9.0\end{array}$ $\begin{array}{lllllll}51372.4 & 51362.1 & 10.3 & 51319.8 & 51310.9 & 8.9\end{array}$ $\begin{array}{lllllll}51361.9 & 51351.7 & 10.2 & 51291.9 & 51283.0 & 8.9\end{array}$ $\begin{array}{lllllll}51374.1 & 51363.9 & 10.2 & 51318.1 & 51309.2 & 8.9\end{array}$ $\begin{array}{llllll}51382.3 & 51372.1 & 10.2 & 51310.4 & 51301.5 & 8.9\end{array}$
VERT. RAD.(nT/m) $-85.9$ $-17.9$ $-51.3$ $-37.7$ $-36.6$ $-36.4$ $-32.6$ $-39.2$ $-35.1$ $-35.7$ $-37.9$ $-36.4$ $-39.2$ $-37.9$ $-33.4$ $-27.7$ $-26.6$ $-13.8$ $-0.2$ 13.6 64.4 148.7 605.4 2169.8 $-62.0$

$-141.8$

$-940.0$

$-313.1$

$-156.6$

$-98.0$

$-71.6$

$-69.2$

$-81.8$

$-72.1$

$-72.8$

$-56.4$

$-45.7$

$-70.5$

$-95.7$

$-74.6$

$-64.1$

$-55.9$

$-22.8$

$-19.8$

$-86.2$

$-114.8$ $-91.8$ $-117.9$ 
TOP

uncorr. corr. dif. uncorr. corr.

$\begin{array}{lllll}51373.1 & 51362.9 & 10.2 & 51317.1 & 51308.3\end{array}$

$\begin{array}{lllllll}51324.3 & 51314.2 & 10.1 & 51140.5 & 51131.7 & 8.8\end{array}$

$\begin{array}{lllllll}51314.9 & 51304.8 & 10.1 & 51193.2 & 51184.4 & 8.8\end{array}$

$\begin{array}{llllllll}51337.3 & 51327.4 & 9.9 & 51301.6 & 51293.1 & 8.5\end{array}$

$\begin{array}{lllllll}51355.3 & 51345.4 & 9.9 & 51341.7 & 51333.2 & 8.5\end{array}$

$\begin{array}{lllllll}51354.0 & 51344.1 & 9.9 & 51330.0 & 51321.5 & 8.5\end{array}$

$\begin{array}{lllllll}51344.8 & 51335.0 & 9.8 & 51322.9 & 51314.5 & 8.4\end{array}$

$\begin{array}{llllllll}51335.7 & 51325.9 & 9.8 & 51319.1 & 51310.7 & 8.4\end{array}$

$\begin{array}{lllllll}51324.8 & 51315.0 & 9.8 & 51300.3 & 51291.9 & 8.4\end{array}$

$\begin{array}{llllllll}51310.7 & 51300.9 & 9.8 & 51282.1 & 51273.8 & 8.3\end{array}$

$\begin{array}{lllllll}51299.0 & 51289.3 & 9.7 & 51272.8 & 51264.5 & 8.3\end{array}$

$\begin{array}{lllllll}51282.7 & 51273.0 & 9.7 & 51257.2 & 51248.9 & 8.3\end{array}$

$\begin{array}{lllllll}51263.7 & 51254.0 & 9.7 & 51235.4 & 51227.1 & 8.3\end{array}$

$\begin{array}{lllllll}51240.9 & 51231.2 & 9.7 & 51212.9 & 51204.7 & 8.2\end{array}$

$\begin{array}{lllllll}51219.5 & 51209.9 & 9.6 & 51191.9 & 51183.7 & 8.2\end{array}$

$\begin{array}{llllll}51192.1 & 51182.5 & 9.6 & 51158.3 & 51150.1 & 8.2\end{array}$

$\begin{array}{lllllll}51153.4 & 51143.8 & 9.6 & 51131.2 & 51123.1 & 8.1\end{array}$

$\begin{array}{lllllll}51090.2 & 51080.6 & 9.6 & 51073.1 & 51065.0 & 8.1\end{array}$

$\begin{array}{lllllll}50978.7 & 50969.2 & 9.5 & 50977.0 & 50968.9 & 8.1\end{array}$

$\begin{array}{lllllll}50844.2 & 50834.7 & 9.5 & 50850.6 & 50842.5 & 8.1\end{array}$

$\begin{array}{lllllll}50622.2 & 50612.7 & 9.5 & 50647.8 & 50639.8 & 8.0\end{array}$

$\begin{array}{lllllll}50295.8 & 50286.3 & 9.5 & 503375.9 & 50367.9 & 8.0\end{array}$

$\begin{array}{lllllll}49864.1 & 49854.7 & 9.4 & 50094.0 & 50086.0 & 8.0\end{array}$

$\begin{array}{llllllll}49260.7 & 49251.3 & 9.4 & 50234.9 & 50227.0 & 7.9\end{array}$

$\begin{array}{lllllll}58608.9 & 58599.5 & 9.4 & 58573.8 & 58565.9 & 7.9\end{array}$

58586.158577 .98 .2

$\begin{array}{lllllll}49956.4 & 49946.7 & 9.7 & 49119.0 & 49110.7 & 8.3\end{array}$

$\begin{array}{lllllll}50720.5 & 50710.8 & 9.7 & 50445.7 & 50437.3 & 8.4\end{array}$

$\begin{array}{lllllll}51072.3 & 51062.6 & 9.7 & 50967.3 & 50958.8 & 8.5\end{array}$

$\begin{array}{lllllll}51213.7 & 51204.0 & 9.7 & 51148.4 & 51139.8 & 8.6\end{array}$

$\begin{array}{lllllll}51275.6 & 51265.8 & 9.8 & 51228.2 & 51219.6 & 8.6\end{array}$

$\begin{array}{llllll}51309.2 & 51299.4 & 9.8 & 51259.9 & 51251.2 & 8.7\end{array}$

$\begin{array}{lllllll}51324.9 & 51315.1 & 9.8 & 51260.4 & 51251.6 & 8.8\end{array}$

$\begin{array}{lllllll}51339.1 & 51329.3 & 9.8 & 51298.7 & 51289.8 & 8.9\end{array}$

$\begin{array}{lllllll}51339.6 & 51329.8 & 9.8 & 51307.2 & 51298.2 & 9.0\end{array}$

$\begin{array}{llllll}51335.3 & 51325.4 & 9.9 & 51286.8 & 51277.7 & 9.1\end{array}$

$\begin{array}{llllll}51345.5 & 51335.6 & 9.9 & 51263.6 & 51254.4 & 9.2\end{array}$

$\begin{array}{lllllll}51363.5 & 51353.6 & 9.9 & 51254.3 & 51245.0 & 9.3\end{array}$

$\begin{array}{llllll}51388.7 & 51378.8 & 9.9 & 51280.2 & 51270.8 & 9.4\end{array}$

$\begin{array}{llllll}51397.2 & 51387.2 & 10.0 & 51329.6 & 51320.2 & 9.4\end{array}$

$\begin{array}{llllll}51397.6 & 51387.6 & 10.0 & 51366.8 & 51357.3 & 9.5\end{array}$

$\begin{array}{llllll}51384.3 & 51374.3 & 10.0 & 51354.4 & 51344.8 & 9.6\end{array}$

$\begin{array}{llllll}51360.5 & 51350.5 & 10.0 & 51307.6 & 51297.9 & 9.7\end{array}$

$\begin{array}{llllll}51339.0 & 51328.9 & 10.1 & 51294.3 & 51284.5 & 9.8\end{array}$

$\begin{array}{llllll}51313.5 & 51303.4 & 10.1 & 51264.8 & 51254.9 & 9.9\end{array}$

$\begin{array}{lllllll}51288.8 & 51278.7 & 10.1 & 51168.0 & 51158.0 & 10.0\end{array}$

$\begin{array}{lllllll}51317.3 & 51307.2 & 10.1 & 51090.8 & 51080.7 & 10.1\end{array}$

$\begin{array}{lllllll}51365.5 & 51355.3 & 10.2 & 51203.2 & 51193.0 & 10.2\end{array}$

$\begin{array}{lllllll}51379.8 & 51369.6 & 10.2 & 51333.1 & 51322.8 & 10.3\end{array}$
VERT.

GRAD.(nT/m)

$-91.8$

$-301.3$

$-199.5$

$-58.5$

$-22.3$

$-39.3$

$-35.9$

$-27.2$

$-40.2$

$-46.9$

$-43.0$

$-41.8$

$-46.4$

$-45.9$

$-45.2$

$-55.4$

$-36.4$

$-28.0$

$-2.8$

10.5

42.0

131.3

376.9

1597.1

$-57.5$

$-1372.8$

$-450.5$

$-172.1$

$-107.0$

$-77.7$

$-80.8$

$-105.7$

$-66.2$

$-53.1$

$-79.5$

$-134.3$

$-179.0$

$-177.9$

$-110.8$

$-50.5$

$-49.0$

$-86.7$

$-73.3$

$-79.8$

$-198.0$

$-371.3$

$-266.1$

$-76.6$ 
Hydroflouric Acid Spill Magnetic Survey Data

TOP

LINE DAY TIME STA. $x$

$\begin{array}{lllll}10 & 261 & 134836 & 1165 & 10\end{array}$

$\begin{array}{llllll}10 & 261 & 134849 & 1167 & 10\end{array}$

$\begin{array}{lllll}10 & 261 & 135015 & 1169 & 10\end{array}$

$\begin{array}{llllll}10 & 261 & 135026 & 1171 & 10\end{array}$

$\begin{array}{lllll}10 & 261 & 135036 & 1173 \quad 10\end{array}$

10

10

10

10

10

10

10

10

10

10

10

10

10

10

10

10

10

10

11

11

11

11

11

11

11

11

11

11

11

11

11

11

11

11

11

11

11

11

11

11

11

11

11
11 $\begin{array}{lllll}261 & 135048 & 1175 & 10 & -29\end{array}$

$\begin{array}{lllll}261 & 135059 & 1177 & 10 & -30\end{array}$

$\begin{array}{llllll}261 & 135111 & 1179 & 10 & -31\end{array}$

$\begin{array}{lllll}261 & 135122 & 1181 & 10 & -32\end{array}$

$\begin{array}{lllll}261 & 135132 & 1183 & 10 & -33\end{array}$

$\begin{array}{lllll}261 & 135143 & 1185 & 10 & -34\end{array}$

$\begin{array}{llllll}261 & 135154 & 1187 & 10 & -35\end{array}$

$\begin{array}{lllll}261 & 135204 & 1189 & 10 & -36\end{array}$

$\begin{array}{lllll}261 & 135216 & 1191 & 10 & -37\end{array}$

$\begin{array}{lllll}261 & 135226 & 1193 & 10 & -38\end{array}$

$\begin{array}{llllll}261 & 135238 & 1195 & 10 & -39\end{array}$

$\begin{array}{llllll}261 & 135250 & 1197 & 10 & -40\end{array}$

$\begin{array}{llllll}261 & 135302 & 1199 & 10 & -41\end{array}$

$\begin{array}{llllll}261 & 135313 & 1201 & 10 & -42\end{array}$

$\begin{array}{llll}261 & 135325 & 1203 & 10\end{array}$

$\begin{array}{lllll}261 & 135337 & 1205 & 10\end{array}$

$\begin{array}{llll}261 & 135348 & 1207 & 10\end{array}$

$\begin{array}{llll}261 & 135400 & 1209 & 10\end{array}$

$\begin{array}{llll}261 & 135614 & 1221 & 11\end{array}$

$\begin{array}{llll}261 & 135626 & 1223 & 11\end{array}$

$\begin{array}{llll}261 & 135638 & 1225 & 11\end{array}$

$\begin{array}{llll}261 & 135651 & 1227 & 11\end{array}$

$\begin{array}{lllll}261 & 135703 & 1229 & 11\end{array}$

$\begin{array}{llll}261 & 135714 & 1231 & 11\end{array}$

$\begin{array}{llll}261 & 135724 & 1233 & 11\end{array}$

$\begin{array}{llll}261 & 135736 & 1235 & 11\end{array}$

$\begin{array}{llll}261 & 135747 & 1237 & 11\end{array}$

$\begin{array}{llll}261 & 135759 & 1239 & 11\end{array}$

$\begin{array}{llll}261 & 135811 & 1241 & 11\end{array}$

$\begin{array}{llll}261 & 135823 & 1243 & 11\end{array}$

$\begin{array}{lllll}261 & 135836 & 1245 & 11\end{array}$

$\begin{array}{lllll}261 & 135850 & 1247 & 11\end{array}$

$\begin{array}{lllll}261 & 135902 & 1249 & 11\end{array}$

$\begin{array}{llll}261 & 135915 & 1251 & 11\end{array}$

$\begin{array}{llll}261 & 135926 & 1253 & 11\end{array}$

$\begin{array}{llll}261 & 135939 & 1255 & 11\end{array}$

$\begin{array}{llll}261 & 135951 & 1257 & 11\end{array}$

$\begin{array}{llll}261 & 140002 & 1259 & 11\end{array}$

$\begin{array}{llll}261 & 140014 & 1261 & 11\end{array}$

$\begin{array}{llll}261 & 140025 & 1263 & 11\end{array}$

$\begin{array}{llll}261 & 140037 & 1265 & 11\end{array}$

$\begin{array}{llll}261 & 140047 \quad 1267 \quad 11\end{array}$

$\begin{array}{llll}261 & 140057 & 1269 & 11\end{array}$

$\begin{array}{llll}261 & 140109 & 1271 & 11\end{array}$
$-43$

$-44$

$-45$

$-46$

0

$-1$

$-2$

$-3$

$-4$

$-5$

$-6$

$-7$

$-8$

$-9$

$-10$

$-11$

$-12$

$-13$

$-14$

$-15$

$-16$

$-17$

$-18$

$-19$

$-20$

$-21$

$-22$

$-23$

$-24$

$-25$
BOTTOM

uncorr. corr. dif. uncorr. corr.

$\begin{array}{lllllll}51349.9 & 51339.7 & 10.2 & 51275.4 & 51265.0 & 10.4 & -122.1\end{array}$

$\begin{array}{lllllll}51308.8 & 51298.5 & 10.3 & 51175.8 & 51165.3 & 10.5 & -218.0\end{array}$

$\begin{array}{lllllll}51322.7 & 51312.3 & 10.4 & 51260.0 & 51248.8 & 11.2 & -102.8\end{array}$

$\begin{array}{lllllll}51333.6 & 51323.1 & 10.5 & 51307.1 & 51295.8 & 11.3 & -43.4\end{array}$

$\begin{array}{lllllll}51331.2 & 51320.7 & 10.5 & 51292.7 & 51281.3 & 11.4 & -63.1\end{array}$

$\begin{array}{lllllll}51325.0 & 51314.5 & 10.5 & 51287.6 & 51276.1 & 11.5 & -61.3\end{array}$

$\begin{array}{lllllll}51311.5 & 51301.0 & 10.5 & 51281.7 & 51270.2 & 11.5 & -48.9\end{array}$

$\begin{array}{llllllll}51304.0 & 51293.4 & 10.6 & 51260.9 & 51249.3 & 11.6 & -70.7\end{array}$

$\begin{array}{lllllll}51289.2 & 51278.6 & 10.6 & 51238.6 & 51226.9 & 11.7 & -82.9\end{array}$

$\begin{array}{llllllll}51270.2 & 51259.6 & 10.6 & 51227.0 & 51215.2 & 11.8 & -70.8\end{array}$

$\begin{array}{llllllll}51251.1 & 51240.5 & 10.6 & 51212.0 & 51200.1 & 11.9 & -64.1\end{array}$

$\begin{array}{lllllll}51229.7 & 51219.1 & 10.6 & 51190.8 & 51178.8 & 12.0 & -63.8\end{array}$

$\begin{array}{llllllll}51202.3 & 51191.6 & 10.7 & 51167.6 & 51155.5 & 12.1 & -56.9\end{array}$

$\begin{array}{lllllll}51173.1 & 51162.4 & 10.7 & 51131.9 & 51119.8 & 12.1 & -67.5\end{array}$

$\begin{array}{llllllll}51142.8 & 51132.1 & 10.7 & 51097.4 & 51085.2 & 12.2 & -74.4\end{array}$

$\begin{array}{lllllll}51124.5 & 51113.8 & 10.7 & 51128.2 & 51115.9 & 12.3 & 6.1\end{array}$

$\begin{array}{llllllll}51056.4 & 51045.6 & 10.8 & 51087.7 & 51075.3 & 12.4 & 51.3\end{array}$

$\begin{array}{llllllll}50905.6 & 50894.8 & 10.8 & 50883.6 & 50871.1 & 12.5\end{array}$

$\begin{array}{lllllll}50685.0 & 50674.2 & 10.8 & 50702.1 & 50689.5 & 12.6\end{array}$

$\begin{array}{llllllll}50456.7 & 50445.9 & 10.8 & 50466.5 & 50453.8 & 12.7\end{array}$

$\begin{array}{lllllll}50016.9 & 50006.0 & 10.9 & 50146.8 & 50134.0 & 12.8\end{array}$

$\begin{array}{lllllll}49227.9 & 49217.0 & 10.9 & 49662.5 & 496.69 .6 & 12.9\end{array}$

$\begin{array}{llllll}48466.1 & 48455.2 & 10.9 & 51461.3 & 51448.4 & 12.9\end{array}$

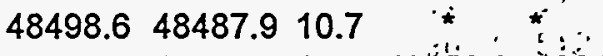

$\begin{array}{llllll}49819.8 & 49809.2 & 10.6 & 490058.3 & 49044.7 & 13.6\end{array}$

$\begin{array}{lllllll}50680.1 & 50669.5 & 10.6 & 50472.9 & 50459.3 & 13.6\end{array}$

$\begin{array}{llllll}51042.5 & 51031.9 & 10.6 & 50934.2 & 509.20 .5 & 13.7\end{array}$

$\begin{array}{lllllll}51204.8 & 51194.2 & 10.6 & 51136.0 & 51122.3 & 13.7\end{array}$

$\begin{array}{lllllll}51263.2 & 51252.6 & 10.6 & 51217.6 & 51203.8 & 13.8\end{array}$

$\begin{array}{llllllll}51299.2 & 51288.6 & 10.6 & 51252.6 & 51238.8 & 13.8\end{array}$

$\begin{array}{lllllll}51319.7 & 51309.1 & 10.6 & 512277.8 & 51263.9 & 13.9\end{array}$

$\begin{array}{llllllll}51325.6 & 51315.0 & 10.6 & 51331.3 & 51317.4 & 13.9\end{array}$

$\begin{array}{lllllll}51298.9 & 51288.3 & 10.6 & 51252.2 & 51238.2 & 14.0\end{array}$

$\begin{array}{lllllll}51256.6 & 51246.0 & 10.6 & 51144.3 & 51130.3 & 14.0\end{array}$

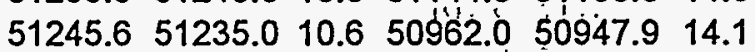

$\begin{array}{llllll}51356.2 & 51345.6 & 10.6 & 50866.5 & 50852.4 & 14.1\end{array}$

$\begin{array}{llllll}51442.0 & 51431.5 & 10.5 & 510004.9 & 50990.7 & 14.2\end{array}$

$\begin{array}{lllllll}51422.3 & 51411.8 & 10.5 & 51207.0 & 511,192.8 & 14.2\end{array}$

$\begin{array}{lllllll}51389.9 & 51379.4 & 10.5 & 51309.2 & 51294.9 & 14.3\end{array}$

$\begin{array}{lllllll}51367.8 & 51357.3 & 10.5 & 51311.2 & 51296.9 & 14.3\end{array}$

$\begin{array}{lllllll}51332.2 & 51321.7 & 10.5 & 51301.0 & 51286.6 & 14.4\end{array}$

$\begin{array}{lllllll}51274.2 & 51263.7 & 10.5 & 51207.0 & 51192.6 & 14.4\end{array}$

$\begin{array}{lllllll}51188.5 & 51178.0 & 10.5 & 51042.5 & 51028.0 & 14.5\end{array}$

$\begin{array}{lllllll}51096.2 & 51085.7 & 10.5 & 50706.3 & 50691.8 & 14.5\end{array}$

$\begin{array}{lllllll}51390.2 & 51379.7 & 10.5 & 50353.3 & 50338.7 & 14.6\end{array}$

$\begin{array}{lllllll}51583.9 & 51573.4 & 10.5 & 50507.9 & 50493.3 & 14.6\end{array}$

$\begin{array}{lllllll}51440.2 & 51429.7 & 10.5 & 510026.5 & 51011.8 & 14.7\end{array}$

$\begin{array}{lllllll}51364.7 & 51354.2 & 10.5 & 51245.0 & 51230.3 & 14.7\end{array}$

$\begin{array}{llllll}51319.4 & 51308.9 & 10.5 & 51254.8 & 51240.1 & 14.7\end{array}$
$-36.1$

28.0

16.1

212.9

745.2

4910.2

$-1248.4$

$-339.7$

$-177.5$

$-112.8$

$-74.8$

$-76.4$

$-68.7$

9.3

$-76.6$

$-184.1$

$-464.9$

$-802.8$

$-716.6$

$-353.0$

$-132.3$

$-92.8$

$-51.1$

$-110.2$

$-239.3$

$-639.2$

$-1699.8$

$-1763.9$

$-678.2$

$-196.2$

$-105.9$ 
TOP

uncorr. corr. dif. uncorr corr.

LINE DAY TIME STA. $X$ $\begin{array}{lllll}11 & 261 & 140242 & 1273 & 11\end{array}$ $\begin{array}{lllll}11 & 261 & 140253 & 1275 & 11\end{array}$

$\begin{array}{lllll}11 & 261 & 140304 & 1277 & 11\end{array}$

$\begin{array}{lllll}11 & 261 & 140315 & 1279 & 11\end{array}$

$\begin{array}{lllll}11 & 261 & 140327 & 1281 & 11\end{array}$

$\begin{array}{lllll}11 & 261 & 140340 & 1283 & 11\end{array}$

$\begin{array}{lllll}11 & 261 & 140352 & 1285 & 11\end{array}$

$\begin{array}{llllll}11 & 261 & 140403 & 1287 & 11\end{array}$

$\begin{array}{lllll}11 & 261 & 140415 & 1289 & 11\end{array}$

$\begin{array}{lllll}11 & 261 & 140426 & 1291 & 11\end{array}$

$\begin{array}{lllll}11 & 261 & 140437 & 1293 & 11\end{array}$

$\begin{array}{lllll}11 & 261 & 140448 & 1295 & 11\end{array}$

$\begin{array}{lllll}11 & 261 & 140500 & 1297 & 11\end{array}$

$\begin{array}{lllll}11 & 261 & 140511 & 1299 & 11\end{array}$

$\begin{array}{lllll}11 & 261 & 140522 & 1301 & 11\end{array}$

$\begin{array}{lllll}11 & 261 & 140534 & 1303 & 11\end{array}$

$\begin{array}{lllll}11 & 261 & 140547 & 1305 & 11\end{array}$

$\begin{array}{lllll}11 & 261 & 140604 & 1307 & 11\end{array}$

$\begin{array}{lllll}11 & 261 & 140616 & 1309 & 11\end{array}$

$\begin{array}{llllll}11 & 261 & 140628 & 1311 & 11\end{array}$

$\begin{array}{lllll}12 & 261 & 140846 & 1322 & 12\end{array}$

$\begin{array}{lllll}12 & 261 & 140902 & 1324 & 12\end{array}$

$\begin{array}{lllll}12 & 261 & 140914 & 1326 & 12\end{array}$

$\begin{array}{lllll}12 & 261 & 140925 & 1328 & 12\end{array}$

$\begin{array}{lllll}12 & 261 & 140936 & 1330 & 12\end{array}$

$\begin{array}{lllll}12 & 261 & 140946 & 1332 & 12\end{array}$

$\begin{array}{lllll}12 & 261 & 140957 & 1334 & 12\end{array}$

$\begin{array}{lllll}12 & 261 & 141008 & 1336 & 12\end{array}$

$\begin{array}{llllll}12 & 261 & 141019 & 1338 & 12\end{array}$

$\begin{array}{llllll}12 & 261 & 141032 & 1340 & 12\end{array}$

$\begin{array}{lllll}12 & 261 & 141044 & 1342 & 12\end{array}$

$\begin{array}{lllll}12 & 261 & 141056 & 1344 & 12\end{array}$

$\begin{array}{llllll}12 & 261 & 141109 & 1346 & 12\end{array}$

$\begin{array}{lllll}12 & 261 & 141121 & 1348 & 12\end{array}$

$\begin{array}{llllll}12 & 261 & 141134 & 1350 & 12\end{array}$

$\begin{array}{lllll}12 & 261 & 141146 & 1352 & 12\end{array}$

$\begin{array}{llllll}12 & 261 & 141157 & 1354 & 12\end{array}$

$\begin{array}{lllll}12 & 261 & 141208 & 1356 & 12\end{array}$

$\begin{array}{lllll}12 & 261 & 141219 & 1358 & 12\end{array}$

$\begin{array}{llllll}12 & 261 & 141230 & 1360 & 12\end{array}$

$\begin{array}{lllll}12 & 261 & 141242 & 1362 & 12\end{array}$

$\begin{array}{llllll}12 & 261 & 141256 & 1364 & 12\end{array}$

$\begin{array}{llllll}12 & 261 & 141308 & 1366 & 12\end{array}$

$\begin{array}{llllll}12 & 261 & 141320 & 1368 & 12\end{array}$

$\begin{array}{llllll}12 & 261 & 141333 & 1370 & 12\end{array}$

$\begin{array}{llllll}12 & 261 & 141348 & 1372 & 12\end{array}$

$\begin{array}{llllll}12 & 261 & 142013 & 1374 & 12\end{array}$

$\begin{array}{llllll}12 & 261 & 142023 & 1376 & 12\end{array}$

$\begin{array}{lllll}12 & 261 & 142033 & 1378 & 12\end{array}$
$Y$

$-26$

$-27$

$-28$

$-29$

$-30$

$-31$

$-32$

$-33$

$-34$

$-35$

$-36$

$-37$

$-38$

$-39$

$-40$

$-41$

$-42$

$-43$

$-44$

$-45$

0

$-1$

$-2$

$-3$

$-4$

$-5$

$-6$

$-7$

$-8$

$-9$

$-10$

$-11$

$-12$

$-13$

$-14$

$-15$

$-16$

$-17$

$-18$

$-19$

$-20$

$-21$

$-22$

$-23$

$-24$

$-25$

$-26$

$-27$

$-28$ $\begin{array}{llllll}51311.5 & 51301.1 & 10.4 & 51192.1 & 51177.0 & 15.1\end{array}$

$\begin{array}{llllll}51314.6 & 51304.2 & 10.4 & 51230.0 & 51214.8 & 15.2\end{array}$

$\begin{array}{llllll}51304.5 & 51294.1 & 10.4 & 51231.2 & 51216.0 & 15.2\end{array}$

$\begin{array}{lllllll}51295.9 & 51285.5 & 10.4 & 51232.8 & 51217.5 & 15.3\end{array}$

$\begin{array}{lllllll}51286.6 & 51276.2 & 10.4 & 51225.4 & 51210.1 & 15.3\end{array}$

$\begin{array}{lllllll}51274.6 & 51264.3 & 10.3 & 51206.8 & 51191.4 & 15.4\end{array}$

$\begin{array}{lllllll}51260.1 & 51249.8 & 10.3 & 51193.6 & 51178.2 & 15.4\end{array}$

$\begin{array}{lllllll}51240.8 & 51230.5 & 10.3 & 51182.2 & 51166.7 & 15.5\end{array}$

$\begin{array}{lllllll}51217.7 & 51207.4 & 10.3 & 51170.7 & 51155.2 & 15.5\end{array}$

$\begin{array}{lllllll}51191.0 & 51180.7 & 10.3 & 51147.4 & 51131.9 & 15.5\end{array}$

$\begin{array}{lllllll}51160.9 & 51150.6 & 10.3 & 51115.8 & 51100.2 & 15.6\end{array}$

$\begin{array}{llllll}51125.3 & 51115.0 & 10.3 & 51078.4 & 51062.8 & 15.6\end{array}$

$\begin{array}{lllllll}51090.2 & 51079.9 & 10.3 & 51023.6 & 51007.9 & 15.7\end{array}$

$\begin{array}{lllllll}51091.3 & 51081.0 & 10.3 & 51127.0 & 51111.3 & 15.7\end{array}$

$\begin{array}{llllll}51029.2 & 51018.9 & 10.3 & 51402.3 & 51386.5 & 15.8\end{array}$

$\begin{array}{lllllll}50796.1 & 50785.8 & 10.3 & 50767.3 & 50751.5 & 15.8\end{array}$

$\begin{array}{lllllll}50541.0 & 50530.7 & 10.3 & 50482.4 & 50466.5 & 15.9\end{array}$

$\begin{array}{lllllll}50113.1 & 50102.9 & 10.2 & 50147.7 & 50131.8 & 15.9\end{array}$

$\begin{array}{lllllll}49189.3 & 49179.1 & 10.2 & 49804.1 & 49788.1 & 16.0\end{array}$

$\begin{array}{lllllll}52097.7 & 52087.5 & 10.2 & 48837.9 & 48821.9 & 16.0\end{array}$

48631.648618 .213 .4

$\begin{array}{llllll}49995.3 & 49981.9 & 13.4 & 49320.6 & 49306.5 & 14.1\end{array}$

$\begin{array}{lllllll}50663.6 & 50650.3 & 13.3 & 50439.1 & 50425.1 & 14.0\end{array}$

$\begin{array}{lllllll}51029.2 & 51015.9 & 13.3 & 509926.8 & 50912.8 & 14.0\end{array}$

$\begin{array}{lllllll}51178.0 & 51164.7 & 13.3 & 51115.3 & 51101.4 & 13.9\end{array}$

$\begin{array}{lllllll}51251.3 & 51238.0 & 13.3 & 51202.9 & 51189.1 & 13.8\end{array}$

$\begin{array}{lllllll}51288.8 & 51275.5 & 13.3 & 51242.1 & 51228.3 & 13.8\end{array}$

$\begin{array}{lllllll}51309.7 & 51296.5 & 13.2 & 51284.0 & 51270.3 & 13.7\end{array}$

$\begin{array}{llllll}51301.1 & 51287.9 & 13.2 & 51283.2 & 51269.5 & 13.7\end{array}$

$\begin{array}{llllll}51249.0 & 51235.8 & 13.2 & 51201.6 & 51188.0 & 13.6\end{array}$

$\begin{array}{llllll}51138.1 & 51124.9 & 13.2 & 50955.4 & 50941.9 & 13.5\end{array}$

$\begin{array}{lllllll}51066.9 & 51053.7 & 13.2 & 50352.5 & 50339.0 & 13.5\end{array}$

$\begin{array}{lllllll}58622.9 & 58609.8 & 13.1 & 58560.7 & 58547.3 & 13.4\end{array}$

$\begin{array}{lllllll}51938.0 & 51924.9 & 13.1 & 58570.4 & 58557.0 & 13.4\end{array}$

$\begin{array}{lllllll}51534.9 & 51521.8 & 13.1 & 509 ̋ 85.6 & 50972.3 & 13.3\end{array}$

$\begin{array}{llllll}51401.0 & 51387.9 & 13.1 & 51235.7 & 51222.5 & 13.2\end{array}$

$\begin{array}{llllll}51349.5 & 51336.4 & 13.1 & 512 \hat{2} 48.5 & 51235.3 & 13.2\end{array}$

$\begin{array}{lllllll}51301.7 & 51288.7 & 13.0 & 51237.1 & 51224.0 & 13.1\end{array}$

$\begin{array}{lllllll}51218.5 & 51205.5 & 13.0 & 51134.3 & 51121.3 & 13.0\end{array}$

$\begin{array}{lllllll}51054.0 & 51041.0 & 13.0 & 50830.3 & 50817.3 & 13.0\end{array}$

$\begin{array}{llllllll}50753.9 & 50740.9 & 13.0 & 49958.4 & 49945.5 & 12.9\end{array}$

$\begin{array}{llllllll}54640.7 & 54627.7 & 13.0 & 58560.7 & 58547.8 & 12.9\end{array}$

$\begin{array}{lllllll}54816.0 & 54803.1 & 12.9 & 58576.1 & 58563.3 & 12.8\end{array}$

$\begin{array}{lllllll}51721.2 & 51708.3 & 12.9 & 5064 & 45.5 & 50632.8 & 12.7\end{array}$

$\begin{array}{lllllll}51352.7 & 51339.8 & 12.9 & 510024.6 & 51011.9 & 12.7\end{array}$

$\begin{array}{lllllll}51289.8 & 51276.9 & 12.9 & 510014.8 & 51002.2 & 12.6\end{array}$

$\begin{array}{lllllll}51520.6 & 51508.4 & 12.2 & 50943.4 & 50932.8 & 10.6\end{array}$

$\begin{array}{lllllll}51362.3 & 51350.1 & 12.2 & 51208.6 & 51198.1 & 10.5\end{array}$

$\begin{array}{llllll}51266.8 & 51254.6 & 12.2 & 51152.3 & 51141.8 & 10.5\end{array}$
VERT.

GRAD.(nT/m)

$-195.7$

$-138.7$

$-120.2$

$-103.4$

$-100.3$

$-111.1$

$-109.0$

$-96.1$

$-77.0$

$-71.5$

$-73.9$

$-76.9$

$-109.2$

58.5

611.6

$-47.2$

$-96.1$

56.7

1007.9

$-5343.9$

$-1106.1$

$-368.0$

$-167.9$

$-102.8$

$-79.3$

$-76.6$

$-42.1$

$-29.3$

$-77.7$

$-299.5$

$-1171.2$

$-102.0$

10872.8

$-900.5$

$-271.0$

$-165.6$

$-105.9$

$-138.0$

$-366.7$

$-1304.1$

6426.2

6164.1

$-1763.4$

$-537.9$

$-450.8$

$-946.2$

$-252.0$

$-187.7$ 
Hydroflouric Acid Spill Magnetic Survey Data

TOP BOTTOM VERT.

LINE DAY TIME STA $X$

$\begin{array}{lllll}12 & 261 & 142044 & 1380 & 12\end{array}$

$\begin{array}{lllll}12 & 261 & 142056 & 1382 & 12\end{array}$

$\begin{array}{llllll}12 & 261 & 142107 & 1384 & 12\end{array}$

$\begin{array}{lllll}12 & 261 & 142119 & 1386 & 12\end{array}$

$\begin{array}{lllll}12 & 261 & 142129 & 1388 & 12\end{array}$

$\begin{array}{llllll}12 & 261 & 142140 & 1390 & 12\end{array}$

$\begin{array}{llllll}12 & 261 & 142150 & 1392 & 12\end{array}$

$\begin{array}{lllll}12 & 261 & 142203 & 1394 & 12\end{array}$

$\begin{array}{llllll}12 & 261 & 142214 & 1396 & 12\end{array}$

$\begin{array}{llllll}12 & 261 & 142226 & 1398 & 12\end{array}$

$\begin{array}{llllll}12 & 261 & 142237 & 1400 & 12\end{array}$

$\begin{array}{lllll}12 & 261 & 142248 & 1402 & 12\end{array}$

$\begin{array}{lllll}12 & 261 & 142259 & 1404 & 12\end{array}$

$\begin{array}{llllll}12 & 261 & 142310 & 1406 & 12\end{array}$

$\begin{array}{lllll}12 & 261 & 142322 & 1408 & 12\end{array}$

$\begin{array}{llllll}13 & 261 & 143546 & 1430 & 13\end{array}$

$\begin{array}{lllll}13 & 261 & 143559 & 1432 & 13\end{array}$

$\begin{array}{lllll}13 & 261 & 143611 & 1434 & 13\end{array}$

$\begin{array}{lllll}13 & 261 & 143623 & 1436 & 13\end{array}$

13

13

13

13

13

13

13

13

13

13

13

13

13

13

13

13

13

13

13

13

13

13

13

13

13

13

13

13

13

13

\section{$\begin{array}{llll}261 & 143633 & 1438 & 13\end{array}$}

$\begin{array}{llll}261 & 1436431440 & 13\end{array}$

$\begin{array}{llll}261 & 143654 & 1442 & 13\end{array}$

$\begin{array}{llll}261 & 143718 & 1446 & 13\end{array}$

$\begin{array}{llll}261 & 143728 & 1448 & 13\end{array}$

$\begin{array}{llll}261 & 143740 & 1450 & 13\end{array}$

$\begin{array}{llll}261 & 143751 & 1452 & 13\end{array}$

$\begin{array}{llll}261 & 143803 & 1454 & 13\end{array}$

$\begin{array}{llll}261 & 143815 & 1456 & 13\end{array}$

$\begin{array}{llll}261 & 143837 & 1460 & 13\end{array}$

$\begin{array}{llll}261 & 143849 & 146213\end{array}$

$\begin{array}{llll}261 & 143859 & 1464 & 13\end{array}$

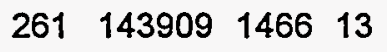

$\begin{array}{llll}261 & 143921 & 1468 & 13\end{array}$

$\begin{array}{llll}261 & 143932 & 1470 & 13\end{array}$

$\begin{array}{llll}261 & 143943 & 1472 & 13\end{array}$

$\begin{array}{lllll}261 & 143953 & 1474 & 13\end{array}$

$\begin{array}{llll}261 & 144003 & 1476 & 13\end{array}$

$\begin{array}{llll}261 & 144014 & 1478 & 13\end{array}$

$\begin{array}{lllll}261 & 144026 & 1480 & 13\end{array}$

$\begin{array}{llll}261 & 144155 & 1482 & 13\end{array}$

$\begin{array}{lllll}261 & 144207 & 1484 & 13\end{array}$

$\begin{array}{lllll}261 & 144214 & 1486 & 13\end{array}$

$\begin{array}{llll}261 & 144226 & 1488 & 13\end{array}$

$\begin{array}{llll}261 & 144239 & 1490 & 13\end{array}$

$\begin{array}{llll}261 & 144250 & 1492 \quad 13\end{array}$

$261144301 \quad 1494 \quad 13$

$\begin{array}{lllll}261 & 144313 & 1496 & 13 & -33\end{array}$ $\begin{array}{llll}261 & 143706 & 1444 & 13\end{array}$

$\begin{array}{lllll}261 & 143826 & 1458 & 13\end{array}$ uncorr. corr. dif. uncorr. corr.

$\begin{array}{lllllll}51248.9 & 51236.8 & 12.1 & 51148.3 & 51137.9 & 10.4 & -164.9\end{array}$

$\begin{array}{lllllll}51250.2 & 51238.1 & 12.1 & 51088.6 & 51078.2 & 10.4 & -264.9\end{array}$

$\begin{array}{llllllll}51252.0 & 51239.9 & 12.1 & 51023.9 & 51013.6 & 10.3 & -373.9\end{array}$

$\begin{array}{lllllll}51236.9 & 51224.8 & 12.1 & 51029.6 & 51019.3 & 10.3 & -339.8\end{array}$

$\begin{array}{lllllll}51190.9 & 51178.8 & 12.1 & 51055.2 & 51045.0 & 10.2 & -222.5\end{array}$

$\begin{array}{lllllll}51146.2 & 51134.2 & 12.0 & 51047.4 & 51037.3 & 10.1 & -162.0\end{array}$

$\begin{array}{lllllll}51101.9 & 51089.9 & 12.0 & 51020.3 & 51010.2 & 10.1 & -133.8\end{array}$

$\begin{array}{lllllll}51049.8 & 51037.8 & 12.0 & 50976.5 & 50966.5 & 10.0 & -120.2\end{array}$

$\begin{array}{lllllll}50995.1 & 50983.1 & 12.0 & 50916.6 & 50906.6 & 10.0 & -128.7\end{array}$

$\begin{array}{lllllll}50919.7 & 50907.7 & 12.0 & 50820.7 & 50810.8 & 9.9 & -162.3\end{array}$

$\begin{array}{lllllll}50852.8 & 50840.9 & 11.9 & 50709.1 & 50699.2 & 9.9 & -235.6\end{array}$

$\begin{array}{lllllll}50750.1 & 50738.2 & 11.9 & 50513.0 & 50503.2 & 9.8 & -388.7\end{array}$

$\begin{array}{llllllll}50476.6 & 50464.7 & 11.9 & 50298.3 & 50288.6 & 9.7 & -292.3\end{array}$

$\begin{array}{llllllll}50020.5 & 50008.6 & 11.9 & 49908.3 & 49898.6 & 9.7 & -183.9\end{array}$

$\begin{array}{llllllll}49547.8 & 49535.9 & 11.9 & 49122.1 & 49112.5 & 9.6 & -697.9\end{array}$

$\begin{array}{llllllll}58609.8 & 58595.4 & 14.4 & 58566.3 & 58560.8 & 5.5 & -71.3\end{array}$

$\begin{array}{llllllll}49843.1 & 49828.8 & 14.3 & 48440.3 & 48434.7 & 5.6 & -2299.7\end{array}$

$\begin{array}{llllllll}50658.1 & 50643.8 & 14.3 & 50349.2 & 50343.5 & 5.7 & -506.4\end{array}$

$\begin{array}{lllllll}51020.4 & 51006.2 & 14.2 & 50911.9 & 50906.0 & 5.9 & -177.9\end{array}$

$\begin{array}{lllllll}51180.4 & 51166.2 & 14.2 & 51117.0 & 51111.0 & 6.0 & -103.9\end{array}$

$\begin{array}{lllllll}51246.9 & 51232.7 & 14.2 & 51202.1 & 51196.0 & 6.1 & -73.4\end{array}$

$\begin{array}{lllllll}51283.9 & 51269.8 & 14.1 & 512400.7 & 51234.5 & 6.2 & -70.8\end{array}$

$\begin{array}{lllllll}51295.5 & 51281.4 & 14.1 & 51273.2 & 51266.9 & 6.3 & -36.6\end{array}$

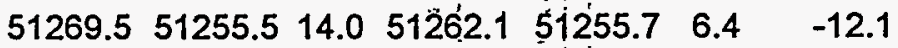

$\begin{array}{lllllll}51182.2 & 51168.2 & 14.0 & 51135.1 & 51128.6 & 6.5 & -77.2\end{array}$

$\begin{array}{llllllll}50930.3 & 50916.3 & 14.0 & 5069 & 98.4 & 50691.8 & 6.6 & -380.2\end{array}$

$\begin{array}{llllllll}50285.8 & 50271.9 & 13.9 & 49204.3 & 49197.5 & 6.8 & -1773.0\end{array}$

$\begin{array}{llllllll}58613.1 & 58599.2 & 13.9 & 58558.2 & 58551.3 & 6.9 & -90.0\end{array}$

$\begin{array}{lllllll}51657.1 & 51643.3 & 13.8 & 50840.5 & 50 & 5 & 5 \\ 5 & 133.4 & 7.1 & -1338.7\end{array}$

$\begin{array}{lllllll}51365.5 & 51351.7 & 13.8 & 51127.0 & 51119.8 & 7.2 & -391.0\end{array}$

$\begin{array}{lllllll}51318.4 & 51304.7 & 13.7 & 51222.9 & 51215.6 & 7.3 & -156.6\end{array}$

$\begin{array}{lllllll}51274.8 & 51261.1 & 13.7 & 51214.9 & 51207.5 & 7.4 & -98.2\end{array}$

$\begin{array}{llllllll}51191.7 & 51178.1 & 13.6 & 51124.9 & 51117.4 & 7.5 & -109.5\end{array}$

$\begin{array}{llllllll}50949.8 & 50936.2 & 13.6 & 50773.7 & 50766.1 & 7.6 & -288.7\end{array}$

$\begin{array}{llllllll}50404.8 & 50391.2 & 13.6 & 49639.5 & 49631.8 & 7.7 & -1254.6\end{array}$

$\begin{array}{lllllll}58573.7 & 58560.2 & 13.5 & 58611.5 & 58603.6 & 7.9 & 62.0\end{array}$

$\begin{array}{lllllll}51556.7 & 51543.3 & 13.4 & 50553.3 & 50545.2 & 8.1 & -1644.9\end{array}$

$\begin{array}{lllllll}51292.0 & 51278.6 & 13.4 & 51000.5 & 50992.3 & 8.2 & -477.9\end{array}$

$\begin{array}{lllllll}51165.0 & 51151.6 & 13.4 & 507833.1 & 500774.8 & 8.3 & -626.1\end{array}$

52022.252009 .113 .1

$\begin{array}{lllllll}51291.6 & 51278.6 & 13.0 & 51070.7 & 51061.4 & 9.3 & -362.1\end{array}$

$\begin{array}{lllllll}51217.7 & 51204.7 & 13.0 & 510 \dot{8} 81.5 & 510072.1 & 9.4 & -223.3\end{array}$

$\begin{array}{lllllll}51188.9 & 51176.0 & 12.9 & 509 \dot{8} 7.9 & 50978.4 & 9.5 & -329.5\end{array}$

$\begin{array}{lllllll}51294.1 & 51281.2 & 12.9 & 50671.7 & 50662.1 & 9.6 & -1020.3\end{array}$

$\begin{array}{lllllll}51528.1 & 51515.2 & 12.9 & 50758.8 & 50749.1 & 9.7 & -1261.1\end{array}$

$\begin{array}{lllllll}51503.3 & 51490.5 & 12.8 & 50835.6 & 50825.8 & 9.8 & -1094.6\end{array}$

$\begin{array}{lllllll}51215.4 & 51202.6 & 12.8 & 508035.7 & 50825.8 & 9.9 & -622.5\end{array}$
$58648.558634 .713 .8 \quad * *$ * * * * * *

$\begin{array}{lllllll}52164.8 & 52151.3 & 13.5 & 58610.1 & 58602.1 & 8.0 & 10566.1\end{array}$ 
TOP uncorr corr dif uncorr cOr. dif. uncorr. corr. $\begin{array}{llllll}51057.2 & 51044.5 & 12.7 & 50886.9 & 50876.8 & 10.1\end{array}$ $\begin{array}{llllll}50953.5 & 50940.8 & 12.7 & 50792.5 & 50782.3 & 10.2\end{array}$ $\begin{array}{lllllll}50882.4 & 50869.7 & 12.7 & 50826.9 & 50816.6 & 10.3\end{array}$ $\begin{array}{lllllll}50778.0 & 50765.4 & 12.6 & 50643.5 & 50633.1 & 10.4\end{array}$ $\begin{array}{llllll}50613.7 & 50601.1 & 12.6 & 50295.0 & 50284.5 & 10.5\end{array}$ $\begin{array}{llllll}50451.0 & 50438.5 & 12.5 & 50127.9 & 50117.3 & 10.6\end{array}$ $\begin{array}{lllllll}50319.1 & 50306.6 & 12.5 & 49964.9 & 4.9954 .2 & 10.7\end{array}$ $\begin{array}{lllllll}49952.8 & 49940.3 & 12.5 & 49636.6 & 49625.8 & 10.8\end{array}$ $\bullet * \quad \div \quad 52059.252056 .62 .6$ $\begin{array}{lllllll}49770.3 & 49772.1 & -1.8 & 48957.9 & 48955.1 & 2.8\end{array}$ $\begin{array}{lllllll}50637.8 & 50639.6 & -1.8 & 50379.5 & 50376.4 & 3.1\end{array}$ $\begin{array}{lllllll}51014.4 & 51016.3 & -1.9 & 50904.3 & 50900.9 & 3.4\end{array}$ $\begin{array}{lllllll}51159.3 & 51161.3 & -2.0 & 51097.6 & 51094.0 & 3.6\end{array}$ $\begin{array}{llllll}51228.6 & 51230.6 & -2.0 & 51181.9 & 51178.0 & 3.9\end{array}$ $\begin{array}{lllllll}51257.5 & 51259.6 & -2.1 & 51219.5 & 51215.4 & 4.1\end{array}$ $\begin{array}{lllllll}51263.3 & 51265.5 & -2.2 & 51221.9 & 51217.5 & 4.4\end{array}$ $\begin{array}{lllllll}51242.9 & 51245.2 & -2.3 & 51180.9 & 51176.3 & 4.6\end{array}$ $\begin{array}{llllll}51182.8 & 51185.1 & -2.3 & 51151.2 & 51146.3 & 4.9\end{array}$ $\begin{array}{lllllll}51005.0 & 51007.4 & -2.4 & 50888.3 & 50883.2 & 5.1\end{array}$ $\begin{array}{lllllll}50513.0 & 50515.5 & -2.5 & 49912.7 & 49907.3 & 5.4\end{array}$ $\begin{array}{lllllll}49997.9 & 50000.4 & -2.5 & 52055.5 & 52049.8 & 5.7\end{array}$ $\begin{array}{lllllll}51123.5 & 51126.1 & -2.6 & 52112.4 & 52106.5 & 5.9\end{array}$ $\begin{array}{lllllll}51394.9 & 51397.6 & -2.7 & 50554.9 & 50548.8 & 6.1\end{array}$ $\begin{array}{llllll}51308.1 & 51310.8 & -2.7 & 51071.4 & 51065.0 & 6.4\end{array}$ $\begin{array}{lllllll}51301.3 & 51304.1 & -2.8 & 51207.9 & 51201.3 & 6.6\end{array}$ $\begin{array}{lllllll}51283.9 & 51286.8 & -2.9 & 51239.5 & 51232.6 & 6.9\end{array}$ $\begin{array}{lllllll}51226.0 & 51228.9 & -2.9 & 51202.2 & 51195.1 & 7.1\end{array}$ $\begin{array}{llllll}51118.4 & 51121.4 & -3.0 & 51045.9 & 51038.5 & 7.4\end{array}$ $\begin{array}{llllll}50892.9 & 50896.0 & -3.1 & 50667.2 & 50659.5 & 7.7\end{array}$ $\begin{array}{llllll}50437.9 & 50441.1 & -3.2 & 50036.8 & 50028.8 & 8.0\end{array}$ $\begin{array}{lllllll}50857.9 & 50861.1 & -3.2 & 49821.7 & 49813.5 & 8.2\end{array}$ $\begin{array}{llllll}51296.9 & 51300.2 & -3.3 & 50491.0 & 50482.4 & 8.6\end{array}$ $\begin{array}{lllllll}51244.8 & 51248.2 & -3.4 & 51073.2 & 51064.3 & 8.9\end{array}$ $\begin{array}{llllll}51218.6 & 51222.1 & -3.5 & 51077.6 & 51068.5 & 9.1\end{array}$ $\begin{array}{lllllll}51197.0 & 51201.1 & -4.1 & 51005.2 & 50993.7 & 11.5\end{array}$ $\begin{array}{lllllll}51217.3 & 51221.5 & -4.2 & 51071.9 & 51060.0 & 11.9\end{array}$ $\begin{array}{llllll}51158.5 & 51162.8 & -4.3 & 51025.7 & 51013.6 & 12.1\end{array}$ $\begin{array}{lllllll}51099.1 & 51103.5 & -4.4 & 50722.1 & 50709.7 & 12.4\end{array}$ $\begin{array}{lllllll}51465.3 & 51469.8 & -4.5 & 49943.7 & 49931.0 & 12.7\end{array}$ $52263.652268 .1 \quad-4.5$

$\begin{array}{llllll}53087.1 & 53091.7 & -4.6 & 52074.9 & 52061.7 & 13.2\end{array}$ $\begin{array}{lllllll}51620.9 & 51625.6 & -4.7 & 50819.9 & 50806.4 & 13.5\end{array}$ $\begin{array}{lllllll}51028.1 & 51032.8 & -4.7 & 50717.9 & 50704.2 & 13.7\end{array}$ $\begin{array}{lllllll}50840.7 & 50845.5 & -4.8 & 50618.0 & 50604.0 & 14.0\end{array}$ $\begin{array}{lllllll}50719.0 & 50723.9 & -4.9 & 50328.0 & 50313.7 & 14.3\end{array}$ $\begin{array}{lllllll}50573.3 & 50578.3 & -5.0 & 50248.6 & 50234.1 & 14.5\end{array}$ $\begin{array}{lllllll}50554.2 & 50559.2 & -5.0 & 49694.3 & 49679.5 & 14.8\end{array}$ $\begin{array}{llllllll}50129.6 & 50134.7 & -5.1 & 48848.7 & 48833.6 & 15.1\end{array}$ $\begin{array}{lllllll}49844.8 & 49850.0 & -5.2 & 483369.4 & 48354.0 & 15.4\end{array}$
VERT. GRAD.(nT/m)

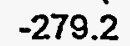


LINE DAY TIME STA. $X$ $\begin{array}{lllll}15 & 262 & 122638 & 1655 & 15\end{array}$

$\begin{array}{lllll}15 & 262 & 122652 & 1657 & 15\end{array}$

$\begin{array}{llllll}15 & 262 & 122708 & 1659 & 15\end{array}$

$\begin{array}{lllll}15 & 262 & 122723 & 1661 & 15\end{array}$

$\begin{array}{lllll}15 & 262 & 122737 & 1663 & 15\end{array}$

$\begin{array}{lllll}15 & 262 & 122751 & 1665 & 15\end{array}$

$\begin{array}{llllll}15 & 262 & 122805 & 1667 & 15\end{array}$

$\begin{array}{lllll}15 & 262 & 122818 & 1669 & 15\end{array}$

$\begin{array}{lllll}15 & 262 & 122831 & 1671 & 15\end{array}$

$\begin{array}{lllll}15 & 262 & 122844 & 1673 & 15\end{array}$

$\begin{array}{lllll}15 & 262 & 122858 & 1675 & 15\end{array}$

$\begin{array}{lllll}15 & 262 & 122912 & 1677 & 15\end{array}$

$\begin{array}{lllll}15 & 262 & 122924 & 1679 & 15\end{array}$

$\begin{array}{lllll}15 & 262 & 122936 & 1681 & 15\end{array}$

$\begin{array}{lllll}15 & 262 & 122948 & 1683 & 15\end{array}$

$\begin{array}{lllll}15 & 262 & 123000 & 1685 & 15\end{array}$

$\begin{array}{lllll}15 & 262 & 123013 & 1687 & 15\end{array}$

$\begin{array}{lllll}15 & 262 & 123026 & 1689 & 15\end{array}$

$\begin{array}{lllll}15 & 262 & 123039 & 1691 & 15\end{array}$

$\begin{array}{lllll}15 & 262 & 123053 & 1693 & 15\end{array}$

$\begin{array}{lllll}15 & 262 & 123106 & 1695 & 15\end{array}$

$\begin{array}{lllll}15 & 262 & 123120 & 1697 & 15\end{array}$

$\begin{array}{lllll}15 & 262 & 123134 & 1699 & 15\end{array}$

$\begin{array}{llllll}15 & 262 & 123147 & 1701 & 15\end{array}$

$\begin{array}{lllll}15 & 262 & 123159 & 1703 & 15\end{array}$

$\begin{array}{lllll}15 & 262 & 123213 & 1705 & 15\end{array}$

$\begin{array}{llllll}15 & 262 & 123351 & 1707 & 15\end{array}$

$\begin{array}{lllll}15 & 262 & 123405 & 1709 & 15\end{array}$

$\begin{array}{lllll}15 & 262 & 123418 & 1711 & 15\end{array}$

$\begin{array}{lllll}15 & 262 & 123432 & 1713 & 15\end{array}$

$\begin{array}{lllll}15 & 262 & 123445 & 1715 \quad 15\end{array}$

$\begin{array}{lllll}15 & 262 & 123501 & 1717 & 15\end{array}$

$\begin{array}{lllll}15 & 262 & 123513 & 1719 & 15\end{array}$

$\begin{array}{lllll}15 & 262 & 123527 & 1721 & 15\end{array}$

$\begin{array}{lllll}15 & 262 & 123540 & 1723 & 15\end{array}$

$\begin{array}{lllll}15 & 262 & 123553 & 1725 & 15\end{array}$

$\begin{array}{lllll}15 & 262 & 123607 & 1727 & 15\end{array}$

$\begin{array}{lllll}15 & 262 & 123621 & 1729 & 15\end{array}$

$\begin{array}{lllll}15 & 262 & 123635 & 1731 & 15\end{array}$

$\begin{array}{lllll}16 & 262 & 124117 & 1743 & 16\end{array}$

$\begin{array}{lllll}16 & 262 & 124132 & 1745 & 16\end{array}$

$\begin{array}{llllll}16 & 262 & 124149 & 1747 & 16\end{array}$

$\begin{array}{llllll}16 & 262 & 124203 & 1749 & 16\end{array}$

$\begin{array}{llllll}16 & 262 & 124218 & 1751 & 16\end{array}$

$\begin{array}{llllll}16 & 262 & 124231 & 1753 & 16\end{array}$

$\begin{array}{llllll}16 & 262 & 124244 & 1755 & 16\end{array}$

$\begin{array}{llllll}16 & 262 & 124257 & 1757 & 16\end{array}$

$\begin{array}{llllll}16 & 262 & 124310 & 1759 & 16\end{array}$

$\begin{array}{lllll}16 & 262 & 124323 & 1761 & 16\end{array}$

$Y$
0
-1
-2
-3
-4
-5
-6
-7
-8
-8
-9
-10
-11
-12
-13
-14
-15
-16
-17
-18
-19
-20
-21
-22
-23
-24
-25
-26
-27
-28
-2
-29
-30
-31
-3
-32
-33
-34
-35
-36
-37
-38
0
-1
-2
-3
-4
-5
-1
-1

TOP BOTTOM VERT.

\begin{tabular}{|c|c|c|c|c|c|c|}
\hline \multicolumn{3}{|c|}{ TOP } & \multicolumn{3}{|c|}{ BOTTOM } & \multirow{2}{*}{$\begin{array}{l}\text { VERT. } \\
\text { GRAD.(nT/m) }\end{array}$} \\
\hline $\begin{array}{l}\text { uncorr. } \\
49025.8\end{array}$ & $\begin{array}{c}\text { corr. } \\
49031.1\end{array}$ & $\begin{array}{l}\text { dif. } \\
-5.3\end{array}$ & uncorr. & corr. & dif. & \\
\hline 49934.8 & 49940.1 & -5.3 & 49505.7 & 49489.2 & 16.5 & -703.4 \\
\hline 50683.2 & 50688.4 & -5.2 & 50504.2 & 50487.7 & 16.5 & -293.4 \\
\hline 51014.1 & 51019.3 & -5.2 & 50922.3 & 50905.7 & 16.6 & -150.5 \\
\hline 51164.3 & 51169.4 & -5.1 & 51101.2 & 51084.5 & 16.7 & -103.4 \\
\hline 51227.4 & 51232.5 & -5.1 & 51180.5 & 51163.8 & 16.7 & -76.9 \\
\hline 51256.8 & 51261.8 & -5.0 & 51213.6 & $5: 1196.8$ & 16.8 & -70.8 \\
\hline 51261.1 & 51266.1 & -5.0 & 51220.3 & 51203.4 & 16.9 & -66.9 \\
\hline 51246.8 & 51251.7 & -4.9 & 51187.4 & 51170.5 & 16.9 & -97.4 \\
\hline 51213.9 & 51218.8 & -4.9 & 51243.1 & 51226.1 & 17.0 & 47.9 \\
\hline 51092.1 & 51097.0 & -4.9 & 51015.5 & 50998.4 & 17.1 & -125.6 \\
\hline 50910.0 & 50914.8 & -4.8 & 50687.3 & 50670.2 & 17.1 & -365.1 \\
\hline 50829.1 & 50833.9 & -4.8 & 50351.2 & 50334.0 & 17.2 & -783.4 \\
\hline 51057.7 & 51062.4 & -4.7 & 50534.5 & 50517.3 & 17.2 & -857.7 \\
\hline 51220.6 & 51225.3 & -4.7 & 50946.5 & 50929.2 & 17.3 & -449.3 \\
\hline 51281.4 & 51286.0 & -4.6 & 51171.5 & 51154.2 & 17.3 & -180.2 \\
\hline 51294.4 & 51299.0 & -4.6 & 51239.1 & 51221.7 & 17.4 & -90.7 \\
\hline 51280.1 & 51284.7 & -4.6 & 51251.6 & 51234.1 & 17.5 & -46.7 \\
\hline 51243.1 & 51247.6 & -4.5 & 51206.3 & 51188.8 & 17.5 & -60.3 \\
\hline 51167.8 & 51172.3 & -4.5 & 51104.8 & 51087.2 & 17.6 & -103.3 \\
\hline 51070.2 & 51074.6 & -4.4 & 50954.4 & 50936.7 & 17.7 & -189.8 \\
\hline 51002.7 & 51007.1 & -4.4 & $507 \ddot{87.7}$ & $50 \dot{7} 70.0$ & 17.7 & -352.5 \\
\hline 51076.2 & 51080.5 & -4.3 & 50826.4 & 50808.6 & 17.8 & -409.5 \\
\hline 51167.4 & 51171.7 & -4.3 & $5083 ́ 0.0$ & 50812.1 & 17.9 & -553.1 \\
\hline 51213.6 & 51217.9 & -4.3 & 51019.7 & 51001.8 & 17.9 & -317.9 \\
\hline 51230.0 & 51234.2 & -4.2 & 51155.4 & 51137.4 & 18.0 & -122.3 \\
\hline 51212.7 & 51216.6 & -3.9 & 51142.1 & 51123.7 & 18.4 & -115.7 \\
\hline 51174.8 & 51178.6 & -3.8 & 51090.4 & 510071.9 & 18.5 & -138.4 \\
\hline 51104.3 & 51108.1 & -3.8 & 50949.5 & 50930.9 & 18.6 & -253.8 \\
\hline 50997.1 & 51000.8 & -3.7 & 50506.2 & 50487.6 & 18.6 & -804.8 \\
\hline 51157.9 & 51161.6 & -3.7 & 52114.1 & 52095.4 & 18.7 & 1567.5 \\
\hline 52936.6 & 52940.3 & -3.7 & 51802.7 & 51783.9 & 18.8 & -1858.9 \\
\hline 54066.4 & 54070.0 & -3.6 & $\star$ & 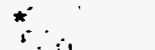 & ? & \\
\hline 51573.9 & 51577.5 & -3.6 & 50507.1 & 50488.2 & 18.9 & -1748.9 \\
\hline 50837.8 & 50841.3 & -3.5 & 50439.9 & 50421.0 & 18.9 & -652.3 \\
\hline 50579.7 & 50583.2 & -3.5 & 50257.7 & 50238.7 & 19.0 & -527.9 \\
\hline 50448.3 & 50451.7 & -3.4 & 49719.2 & 49700.1 & 19.1 & -1195.2 \\
\hline 50258.3 & 50261.7 & -3.4 & 48952.0 & 48932.9 & 19.1 & -2141.5 \\
\hline 50217.6 & 50220.9 & -3.3 & * & $\star$ & 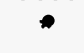 & - \\
\hline 52088.0 & 52089.8 & -1.8 & $\star$ & $\star$ & $\bullet$ & 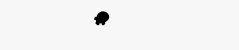 \\
\hline 50030.6 & 50032.3 & -1.7 & 49401.7 & 49385.7 & 16.0 & -1031.0 \\
\hline 50691.6 & 50693.2 & -1.6 & 50511.7 & 50496.0 & 15.7 & -294.9 \\
\hline 51042.0 & 51043.5 & -1.5 & 50934.6 & 50919.2 & 15.4 & -176.1 \\
\hline 51164.8 & 51166.2 & -1.4 & 51088.9 & 51073.8 & 15.1 & -124.4 \\
\hline 51228.4 & 51229.7 & -1.3 & 5117.4 .0 & 51159.2 & 14.8 & -89.2 \\
\hline 51262.4 & 51263.6 & -1.2 & 51204.6 & 51190.0 & 14.6 & -94.8 \\
\hline 51267.4 & 51268.6 & -1.2 & 51214.7 & 51200.4 & 14.3 & -86.4 \\
\hline 51259.5 & 51260.6 & -1.1 & 51205.8 & 51191.8 & 14.0 & -88.0 \\
\hline 51229.9 & 51230.9 & -1.0 & 51190.8 & $51 \nmid 77.0$ & 13.8 & -64.1 \\
\hline
\end{tabular}


TOP

uncorr. corr. dif. uncorr. corr.

$51160.0 \quad 51160.9-0.9$

$51083.351084 .1-0.8$

5127

\section{6}

16

\section{6}

16

16

16

16

16 $\begin{array}{llll}262 & 124458 & 1775 & 16\end{array}$

$\begin{array}{llll}262 & 124511 & 1777 \quad 16\end{array}$

$\begin{array}{llll}262 & 124524 & 1779 & 16\end{array}$

$\begin{array}{llll}262 & 124537 & 1781 & 16\end{array}$

$\begin{array}{llll}262 & 124551 & 1783 & 16\end{array}$

$\begin{array}{llll}262 & 124603 & 1785 & 16\end{array}$

$\begin{array}{llll}262 & 124616 & 1787 & 16\end{array}$

$\begin{array}{llll}262 & 124631 & 1789 & 16\end{array}$

$\begin{array}{llll}262 & 1246451791 & 16\end{array}$

$\begin{array}{llll}262 & 124659 & 1793 & 16\end{array}$

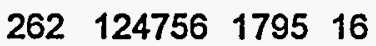

$\begin{array}{llll}262 & 124809 & 1797 & 16\end{array}$

$\begin{array}{llll}262 & 124822 & 1799 & 16\end{array}$

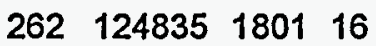

$\begin{array}{llll}262 & 124848 & 1803 & 16\end{array}$

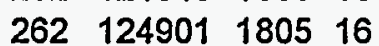

$\begin{array}{lll}262 & 124913 \quad 1807 \quad 16\end{array}$

$\begin{array}{llll}262 & 124927 & 1809 & 16\end{array}$

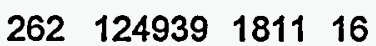

$\begin{array}{llll}262 & 124952 & 1813 & 16\end{array}$

$\begin{array}{lll}262 & 125006 & 181516\end{array}$

$\begin{array}{llll}262 & 125243 & 1827 & 17\end{array}$

$\begin{array}{llll}262 & 125257 & 1829 & 17\end{array}$

$\begin{array}{llll}262 & 125309 & 1831 & 17\end{array}$

$\begin{array}{llll}262 & 125321 & 1833 & 17\end{array}$

$\begin{array}{llll}262 & 125333 & 1835 & 17\end{array}$

$\begin{array}{lllll}262 & 125346 & 1837 & 17\end{array}$

$\begin{array}{llll}262 & 125359 & 1839 & 17\end{array}$

$\begin{array}{lllll}262 & 125414 & 1841 & 17\end{array}$

$\begin{array}{lllll}262 & 125427 & 1843 & 17\end{array}$

$\begin{array}{lllll}262 & 125441 & 1845 & 17\end{array}$

$\begin{array}{lllll}262 & 125454 & 1847 & 17\end{array}$

$\begin{array}{lllll}262 & 125507 & 1849 & 17\end{array}$

$\begin{array}{llll}262 & 125520 & 1851 & 17\end{array}$

$\begin{array}{llll}262 & 125533 & 1853 & 17\end{array}$

$\begin{array}{llll}262 & 125547 & 1855 & 17\end{array}$

$\begin{array}{llll}262 & 125601 & 1857 & 17\end{array}$

$\begin{array}{llll}262 & 125614 & 1859 & 17\end{array}$

$\begin{array}{llll}262 & 125626 & 1861 & 17\end{array}$

$\begin{array}{llll}262 & 125639 & 1863 & 17\end{array}$

$\begin{array}{llll}262 & 125653 & 1865 & 17\end{array}$

$\begin{array}{lllll}262 & 125705 & 1867 & 17\end{array}$

$\begin{array}{llll}262 & 125719 & 1869 & 17\end{array}$
$-16$

$-17$

$-18$

$-19$

$-20$

$-21$

$-22$

$-23$

$-24$

$-25$

$-26$

$-27$

$-28$

$-29$

$-30$

$-31$

$-32$

$-33$

$-34$

$-35$

$-36$

0

$-1$

$-2$

$-3$

$-4$

$-5$

$-6$

$-7$

$-8$

$-9$

$-10$

$-11$

$-12$

$-13$

$-14$

$-15$

$-16$

$-17$

$-18$

$-19$

$-20$

$-21$

\section{$\begin{array}{lllllll}51280.8 & 51281.2 & -0.4 & 51218.0 & 51206.1 & 11.9\end{array}$}

$\begin{array}{lllllll}51273.5 & 51273.8 & -0.3 & 51197.8 & 51186.2 & 11.6\end{array}$

$\begin{array}{lllllll}51253.0 & 51253.3 & -0.3 & 51179.4 & 51168.0 & 11.4\end{array}$

$\begin{array}{llllll}51222.1 & 51222.3 & -0.2 & 51151.5 & 51140.4 & 11.1\end{array}$

$\begin{array}{lllllll}51179.5 & 51179.6 & -0.1 & 51113.7 & 51102.8 & 10.9\end{array}$

$\begin{array}{lllllll}51156.4 & 51156.4 & 0.0 & 51069.9 & 51059.3 & 10.6\end{array}$

$\begin{array}{lllllll}51173.2 & 51173.1 & 0.1 & 51074.7 & 51064.3 & 10.4\end{array}$

$\begin{array}{lllllll}51202.6 & 51202.4 & 0.2 & 51090.7 & 51080.6 & 10.1\end{array}$

$\begin{array}{lllllll}51226.8 & 51226.6 & 0.2 & 51119.1 & 51109.3 & 9.8\end{array}$

$\begin{array}{lllllll}51238.9 & 51238.6 & 0.3 & 51166.1 & 51156.6 & 9.5\end{array}$

$\begin{array}{lllllll}51227.6 & 51226.9 & 0.7 & 51165.6 & 51157.2 & 8.4\end{array}$

$\begin{array}{llllll}51189.7 & 51188.9 & 0.8 & 51121.9 & 51113.8 & 8.1\end{array}$

$\begin{array}{lllllll}51117.2 & 51116.4 & 0.8 & 51017.0 & 51009.1 & 7.9\end{array}$

$\begin{array}{lllllll}51008.9 & 51008.0 & 0.9 & 50791.8 & 50784.2 & 7.6\end{array}$

$\begin{array}{lllllll}50922.4 & 50921.4 & 1.0 & 50159.3 & 50151.9 & 7.4\end{array}$

$\begin{array}{lllllll}51240.7 & 51239.6 & 1.1 & 49748.8 & 499741.7 & 7.1\end{array}$

$\begin{array}{llllll}51258.8 & 51257.6 & 1.2 & 49716.7 & 49709.9 & 6.8\end{array}$

$\begin{array}{lllllll}50850.9 & 50849.7 & 1.2 & 500 \dot{8} 2.3 & 50075.7 & 6.6\end{array}$

$\begin{array}{lllllll}50469.5 & 50468.2 & 1.3 & 50077.1 & 50070.8 & 6.3\end{array}$

$\begin{array}{lllllll}49988.4 & 49987.0 & 1.4 & 49254.6 & 49248.5 & 6.1\end{array}$

$\begin{array}{lllllll}49887.9 & 49886.4 & 1.5 & 45573.2 & 45567.4 & 5.8\end{array}$

$\begin{array}{llllll}50009.8 & 50009.1 & 0.7 & 52058.6 & 52052.2 & 6.4\end{array}$

$\begin{array}{lllllll}50061.4 & 50060.6 & 0.8 & 49501.2 & 49494.6 & 6.6\end{array}$

$\begin{array}{llllllll}50715.9 & 50715.1 & 0.8 & 50479.6 & 50472.9 & 6.7\end{array}$

$\begin{array}{llllllll}51037.7 & 51036.8 & 0.9 & 50921.3 & 50914.4 & 6.9\end{array}$

$\begin{array}{lllllll}51176.9 & 51176.0 & 0.9 & 51070.5 & 51063.4 & 7.1\end{array}$

$\begin{array}{lllllll}51241.9 & 51241.0 & 0.9 & 51161.6 & 51154.4 & 7.2\end{array}$

$\begin{array}{lllllll}51262.4 & 51261.4 & 1.0 & 51205.1 & 51197.7 & 7.4\end{array}$

$\begin{array}{lllllll}51270.3 & 51269.3 & 1.0 & 51218.7 & 51211.1 & 7.6\end{array}$

$\begin{array}{llllll}51266.6 & 51265.5 & 1.1 & 51226.3 & 51218.5 & 7.8\end{array}$

$\begin{array}{llllll}51245.1 & 51244.0 & 1.1 & 51209.9 & 51201.9 & 8.0\end{array}$

$\begin{array}{llllll}51212.1 & 51210.9 & 1.2 & 51148.4 & 51140.3 & 8.1\end{array}$

$\begin{array}{llllll}51172.1 & 51170.9 & 1.2 & 51106.1 & 51097.8 & 8.3\end{array}$

$\begin{array}{llllll}51162.1 & 51160.9 & 1.2 & 51071.3 & 51062.8 & 8.5\end{array}$

$\begin{array}{llllll}51185.4 & 51184.1 & 1.3 & 51065.2 & 51056.5 & 8.7\end{array}$

$\begin{array}{llllll}51223.9 & 51222.6 & 1.3 & 51096.5 & 51087.6 & 8.9\end{array}$

$\begin{array}{llllll}51263.4 & 51262.0 & 1.4 & 51187.2 & 51178.2 & 9.0\end{array}$

$\begin{array}{lllllll}51280.8 & 51279.4 & 1.4 & 51208.1 & 51198.9 & 9.2\end{array}$

$\begin{array}{llllll}51276.6 & 51275.2 & 1.4 & 51207.3 & 51197.9 & 9.4\end{array}$

$\begin{array}{llllll}51270.6 & 51269.1 & 1.5 & 51216.1 & 51206.5 & 9.6\end{array}$

$\begin{array}{lllllll}51257.3 & 51255.8 & 1.5 & 51195.9 & 51186.2 & 9.7\end{array}$

$\begin{array}{lllllll}51243.1 & 51241.5 & 1.6 & 51177.5 & 51167.6 & 9.9\end{array}$

$\begin{array}{lllllll}51237.8 & 51236.2 & 1.6 & 51177.6 & 51167.5 & 10.1 & -98.7\end{array}$
VERT.

RAD.(nT/m)

$-132.5$

$-174.4$

$-285.4$

$-285.9$

$-279.5$

$-152.1$

$-103.0$

$-124.1$

$-120.7$

$-115.7$

$-107.9$

$-141.8$

$-161.5$

$-183.4$

$-176.6$

$-119.3$

$-101.6$

$-111.1$

$-164.3$

$-355.9$

$-1251.0$

$-2445.7$

$-2528.0$

$-1260.0$

$-643.3$

$-1203.0$

$-7073.3$

3358.7

$-918.4$

$-387.4$

$-190.8$

$-174.4$

$-131.6$

$-93.9$

$-84.6$

$-66.1$

$-57.7$

$-104.4$

$-108.2$

$-148.9$

$-197.1$

$-208.9$

$-124.9$

$-119.2$

$-113.6$

$-89.3$

$-100.7$

$-107.5$ 
Hydroflouric Acid Spill Magnetic Survey Data

TOP

LINE DAY TIME STA. $X$

$\begin{array}{lllll}17 & 262 & 125732 & 1871 & 17\end{array}$

$\begin{array}{lllllll}17 & 262 & 125745 & 1873 & 17 & -23\end{array}$

$\begin{array}{llllll}17 & 262 & 125757 & 1875 & 17 & -24\end{array}$

$\begin{array}{llllll}17 & 262 & 125810 & 1877 & 17 & -25\end{array}$

$\begin{array}{llllll}17 & 262 & 125903 & 1879 & 17 & -26\end{array}$

$\begin{array}{llllll}17 & 262 & 125916 & 1881 & 17 & -27\end{array}$

$\begin{array}{llllll}17 & 262 & 125930 & 1883 & 17 & -28\end{array}$

$\begin{array}{llllll}17 & 262 & 125943 & 1885 & 17 & -29\end{array}$

$\begin{array}{llllll}17 & 262 & 125958 & 1887 & 17 & -30\end{array}$

$\begin{array}{lllllll}17 & 262 & 130009 & 1889 & 17 & -31\end{array}$

$\begin{array}{llllll}17 & 262 & 130022 & 1891 & 17\end{array}$

$\begin{array}{lllll}17 & 262 & 130036 & 1893 & 17\end{array}$

$\begin{array}{lllll}17 & 262 & 130048 & 1895 & 17\end{array}$

$\begin{array}{lllll}18 & 262 & 130336 & 1907 & 18\end{array}$

18

18

18

18

18

18

18

18

18

18

18

18

18

18

18

18

18

18

18

18

18

18

18

18

18

18

18

18

18

18

18

18

18

18

19 $\begin{array}{lllll}262 & 130350 & 1909 & 18\end{array}$

$\begin{array}{llll}262 & 130403 & 1911 & 18\end{array}$

$\begin{array}{llll}262 & 130415 & 1913 & 18\end{array}$

$\begin{array}{llll}262 & 130427 & 1915 & 18\end{array}$

$\begin{array}{lllll}262 & 130440 \quad 1917 & 18\end{array}$

$\begin{array}{llll}262 & 130453 & 1919 & 18\end{array}$

$\begin{array}{llll}262 & 130506 & 1921 & 18\end{array}$

$\begin{array}{llll}262 & 130518 & 1923 & 18\end{array}$

$\begin{array}{llll}262 & 130530 & 1925 & 18\end{array}$

$\begin{array}{lllll}262 & 130543 & 1927 & 18\end{array}$

$\begin{array}{llll}262 & 130556 & 1929 & 18\end{array}$

$\begin{array}{lllll}262 & 130608 & 1931 & 18\end{array}$

$\begin{array}{lllll}262 & 130621 & 1933 & 18\end{array}$

$\begin{array}{lllll}262 & 130634 & 1935 & 18\end{array}$

$\begin{array}{lllll}262 & 130647 & 1937 & 18\end{array}$

$\begin{array}{llll}262 & 130701 & 1939 & 18\end{array}$

$\begin{array}{llll}262 & 130715 & 1941 & 18\end{array}$

$\begin{array}{llll}262 & 130728 & 1943 & 18\end{array}$

$\begin{array}{llll}262 & 1307431945 \quad 18\end{array}$

$\begin{array}{lllll}262 & 130757 & 1947 & 18\end{array}$

$\begin{array}{lllll}262 & 130810 & 1949 & 18\end{array}$

$\begin{array}{llll}262 & 130823 & 1951 & 18\end{array}$

$\begin{array}{llll}262 & 130836 & 1953 & 18\end{array}$

$\begin{array}{llll}262 & 130850 & 1955 & 18\end{array}$

$\begin{array}{llll}262 & 130903 & 1957 & 18\end{array}$

$\begin{array}{llll}262 & 131017 & 1959 & 18\end{array}$

$\begin{array}{llll}262 & 131031 & 1961 & 18\end{array}$

$\begin{array}{llll}262 & 131044 & 1963 & 18\end{array}$

$\begin{array}{llll}262 & 131057 & 1965 & 18\end{array}$

$\begin{array}{llll}262 & 131110 & 1967 & 18\end{array}$

$\begin{array}{llll}262 & 131124 & 1969 & 18\end{array}$

$\begin{array}{llll}262 & 131136 & 1971 & 18\end{array}$

$\begin{array}{llll}262 & 131149 & 1973 & 18\end{array}$

$\begin{array}{llll}262 & 131202 & 1975 & 18\end{array}$

$\begin{array}{lll}262 & 1314501987 & 19\end{array}$
$Y$ uncorr. corr.

$\begin{array}{lll}51244.0 & 51242.3 & 1.7\end{array}$

$51253.4 \quad 51251.7 \quad 1.7$

$\begin{array}{llll}51266.1 & 51264.4 & 1.7\end{array}$

$51265.9 \quad 51264.1 \quad 1.8$

$\begin{array}{llll}51236.9 & 51235.0 & 1.9\end{array}$

$\begin{array}{llll}51188.0 & 51186.0 \quad 2.0\end{array}$

$51116.251114 .2 \quad 2.0$

$51017.451015 .3 \quad 2.1$

$\begin{array}{llll}50912.8 & 50910.7 & 2.1\end{array}$

$\begin{array}{llll}50770.3 & 50768.1 & 2.2\end{array}$

$50679.6 \quad 50677.4 \quad 2.2$

50458.150455 .92 .2

$49650.5 \quad 49648.2 \quad 2.3$

$48620.3 \quad 48617.5 \quad 2.8$

$50039.450036 .4 \quad 3.0$

$50811.150808 .0 \quad 3.1$

$\begin{array}{llll}51057.9 & 51054.6 & 3.3\end{array}$

$\begin{array}{llll}51208.6 & 51205.2 \quad 3.4\end{array}$

$\begin{array}{llll}51272.8 & 51269.3 & 3.5\end{array}$

$\begin{array}{llll}51282.4 & 51278.7 & 3.7\end{array}$

$\begin{array}{llll}51276.0 & 51272.2 & 3.8\end{array}$

$\begin{array}{llll}51272.0 & 51268.0 & 4.0\end{array}$

$51255.5 \quad 51251.4 \quad 4.1$

$51229.6 \quad 51225.4 \quad 4.2$

$51206.3 \quad 51201.9 \quad 4.4$

$51196.7 \quad 51192.2 \quad 4.5$

$51205.4 \quad 51200.7 \quad 4.7$

$51233.4 \quad 51228.6 \quad 4.8$

$51270.5 \quad 51265.5 \quad 5.0$

$\begin{array}{llll}51287.3 & 51282.2 & 5.1\end{array}$

$51285.751280 .4 \quad 5.3$

$51285.951280 .5 \quad 5.4$

$51276.9 \quad 51271.3 \quad 5.6$

$51259.2 \quad 51253.5 \quad 5.7$

$51260.1 \quad 51254.2 \quad 5.9$

51271.251265 .26 .0

$51285.1 \quad 51278.9 \quad 6.2$

$51302.751296 .4 \quad 6.3$

$51304.4 \quad 51297.9 \quad 6.5$

$\begin{array}{llll}51280.0 & 51272.7 & 7.3\end{array}$

$\begin{array}{lll}51225.6 & 51218.1 \quad 7.5\end{array}$

$51149.0 \quad 51141.4 \quad 7.6$

$51034.5 \quad 51026.7 \quad 7.8$

$50890.2 \quad 50882.3 \quad 7.9$

$\begin{array}{llll}50727.5 & 50719.4 & 8.1\end{array}$

$\begin{array}{llll}50592.9 & 50584.7 & 8.2\end{array}$

$50262.2 \quad 50253.9 \quad 8.3$

$\begin{array}{llll}49646.1 & 49637.6 \quad 8.5\end{array}$

-34
0
BOTTOM

uncorr. corr.

$\begin{array}{llll}51175.5 & 51165.2 & 10.3 & -112.3\end{array}$

$\begin{array}{llll}51191.7 & 51181.3 & 10.4 & -101.2\end{array}$

$\begin{array}{llll}51211.5 & 51200.9 & 10.6 & -89.5\end{array}$

$\begin{array}{llll}51220.6 & 51209.8 & 10.8 & -74.3\end{array}$

$\begin{array}{llll}51174.0 & 51162.5 & 11.5 & -103.1\end{array}$

$\begin{array}{llll}51141.6 & 51129.9 & 11.7 & -76.1\end{array}$

$51050.0 \quad 51038.2 \quad 11.8 \quad-108.5$

$\begin{array}{llll}50889.5 & 50877.5 & 12.0 & -209.7\end{array}$

$\begin{array}{llll}50687.2 & 50675.0 & 12.2 & -369.8\end{array}$

$\begin{array}{llll}50454.0 & 50441.6 & 12.4 & -518.5\end{array}$

$\begin{array}{llll}50338.1 & 50325.6 & 12.5 & -559.8\end{array}$

$\begin{array}{llll}50173.2 & 50160.5 & 12.7 & -467.1\end{array}$

$\begin{array}{lllll}49150.2 & 49137.3 & 12.9 & -820.2\end{array}$

$\begin{array}{llll}484.99 .8 & 48484.0 & 15.8 & -197.5\end{array}$

$\begin{array}{llll}49753.0 & 49736.9 & 16.1 & -469.5\end{array}$

$\begin{array}{llll}50611.5 & 50595.1 & 16.4 & -327.2\end{array}$

$\begin{array}{llll}50874.2 & 50857.6 & 16.6 & -301.2\end{array}$

$\begin{array}{llll}51039.5 & 51022.7 & 16.8 & -277.2\end{array}$

$\begin{array}{llll}51147.4 & 51130.3 & 17.1 & -205.6\end{array}$

$\begin{array}{llll}51190.6 & 51.173 .3 & 17.3 & -150.5\end{array}$

$\begin{array}{llll}51218.5 & 51201.1 & 17.6 & -93.9\end{array}$

$\begin{array}{llll}51 \text { 19 } 7.1 & 51179.3 & 17.8 & -122.8\end{array}$

$\begin{array}{llll}51206.3 & 51188.2 & 18.1 & -80.7\end{array}$

$\begin{array}{llll}51180.9 & 511,62.6 & 18.3 & -79.8\end{array}$

$\begin{array}{lllll}51142.3 & 51123.7 & 18.6 & -104.9\end{array}$

$51114.3 \quad 51095.5 \quad 18.8 \quad-135.1$

$\begin{array}{llll}51121.8 & 51102.7 & 19.1 & -137.1\end{array}$

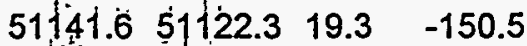

$\begin{array}{llll}51207.2 & 51187.6 & 19.6 & -103.8\end{array}$

$\begin{array}{llll}51217.5 & 51197.6 & 19.9 & -114.4\end{array}$

$\begin{array}{llll}51216.8 & 51196.7 & 20.1 & -112.9\end{array}$

$\begin{array}{llll}51233.5 & 51213.1 & 20.4 & -85.9\end{array}$

$\begin{array}{llll}51226.9 & 51206.2 & 20.7 & -82.0\end{array}$

$\begin{array}{llll}51193.6 & 51172.7 & 20.9 & -107.5\end{array}$

$\begin{array}{llll}51189.6 & 51168.4 & 21.2 & -115.6\end{array}$

$\begin{array}{llll}51199.2 & 51177.7 & 21.5 & -118.0\end{array}$

$\begin{array}{llll}51.204 .5 & 51182.8 & 21.7 & -132.1\end{array}$

$51208.1 \quad 51186.122 .0 \quad-155.1$

$\begin{array}{llll}51252.1 & 51229.8 & 22.3 & -85.7\end{array}$

$\begin{array}{llll}51232.9 & 51209.2 & 23.7 & -77.2\end{array}$

$\begin{array}{llll}51.81 .8 & 51167.8 & 24.0 & -55.4\end{array}$

$51105.451081 .2 \quad 24.2 \quad-71.5$

$\begin{array}{llll}50952.6 & 50928.1 & 24.5 & -134.3\end{array}$

$\begin{array}{llll}50764.4 & 50739.6 & 24.8 & -206.2\end{array}$

$\begin{array}{llll}50578.0 & 50553.0 & 25.0 & -245.1\end{array}$

$\begin{array}{llll}50409.4 & 503 \dot{8} 8.1 & 25.3 & -300.8\end{array}$

$\begin{array}{llll}50046.0 & 50020.5 & 25.5 & -354.4\end{array}$

$\begin{array}{llll}49285.8 & 49260.0 & 25.8 & -590.7\end{array}$

* $\quad 477977.747769 .827 .9$

Page 33 
Hydroflouric Acid Spill Magnetic Survey Data

TOP

LINE DAY TIME STA. $X \quad Y$

$\begin{array}{llllll}19 & 262 & 131504 & 1989 & 19 & -1\end{array}$

$\begin{array}{llllll}19 & 262 & 131515 & 1991 & 19 & -2\end{array}$

$\begin{array}{llllll}19 & 262 & 131527 & 1993 & 19\end{array}$

$\begin{array}{lllll}19 & 262 & 131540 & 1995 & 19\end{array}$

$\begin{array}{lllll}19 & 262 & 131552 & 1997 & 19\end{array}$

$\begin{array}{lllll}19 & 262 & 131603 & 1999 & 19\end{array}$

$19 \quad 262 \quad 131616 \quad 2001 \quad 19$

$\begin{array}{lllll}19 & 262 & 131629 & 2003 & 19\end{array}$

$\begin{array}{lllll}19 & 262 & 131642 & 2005 & 19\end{array}$

$\begin{array}{lllll}19 & 262 & 131658 & 2007 & 19\end{array}$

$\begin{array}{lllll}19 & 262 & 131713 & 2009 & 19\end{array}$

$\begin{array}{lllll}19 & 262 & 131726 & 2011 & 19\end{array}$

$\begin{array}{lllll}19 & 262 & 131738 & 2013 & 19\end{array}$

$\begin{array}{lllll}19 & 262 & 131755 & 2015 & 19\end{array}$

$\begin{array}{lllll}19 & 262 & 131810 & 2017 & 19\end{array}$

$\begin{array}{lllll}19 & 262 & 131824 & 2019 & 19\end{array}$

$\begin{array}{lllll}19 & 262 & 131840 & 2021 & 19\end{array}$

$\begin{array}{lllll}19 & 262 & 131854 & 2023 & 19\end{array}$

$\begin{array}{lllll}19 & 262 & 131908 & 2025 & 19\end{array}$

$\begin{array}{lllll}19 & 262 & 131922 & 2027 & 19\end{array}$

$19 \quad 262 \quad 131936 \quad 2029 \quad 19$

$\begin{array}{lllll}19 & 262 & 131949 & 2031 & 19\end{array}$

$\begin{array}{lllll}19 & 262 & 132004 & 2033 & 19\end{array}$

$\begin{array}{lllll}19 & 262 & 132020 & 2035 & 19\end{array}$

$\begin{array}{lllll}19 & 262 & 132033 & 2037 & 19\end{array}$

$\begin{array}{lllll}19 & 262 & 132145 & 2039 & 19\end{array}$

$\begin{array}{lllll}19 & 262 & 132158 & 2041 & 19\end{array}$

$\begin{array}{lllll}19 & 262 & 132212 & 2043 & 19\end{array}$

$\begin{array}{lllll}19 & 262 & 132227 & 2045 & 19\end{array}$

$\begin{array}{lllll}19 & 262 & 132240 & 2047 & 19\end{array}$

$\begin{array}{lllll}19 & 262 & 132253 & 2049 & 19\end{array}$

$\begin{array}{lllll}19 & 262 & 132305 & 2051 & 19\end{array}$

$\begin{array}{lllll}19 & 262 & 132317 & 2053 & 19\end{array}$

$\begin{array}{lllll}20 & 262 & 132708 & 2065 & 20\end{array}$

$\begin{array}{lllll}20 & 262 & 132723 & 2067 & 20\end{array}$

$\begin{array}{lllll}20 & 262 & 132737 & 2069 & 20\end{array}$

$20 \quad 262 \quad 132750 \quad 2071 \quad 20$

$\begin{array}{lllll}20 & 262 & 132802 & 2073 & 20\end{array}$

$\begin{array}{lllll}20 & 262 & 132816 & 2075 & 20\end{array}$

$\begin{array}{lllll}20 & 262 & 132828 & 2077 & 20\end{array}$

$\begin{array}{lllll}20 & 262 & 132843 & 2079 & 20\end{array}$

$\begin{array}{lllll}20 & 262 & 132856 & 2081 & 20\end{array}$

$\begin{array}{lllll}20 & 262 & 132907 & 2083 & 20\end{array}$

$\begin{array}{lllll}20 & 262 & 132918 & 2085 & 20\end{array}$

$\begin{array}{lllll}20 & 262 & 132932 & 2087 & 20\end{array}$

$\begin{array}{lllll}20 & 262 & 132944 & 2089 & 20\end{array}$

$\begin{array}{llllll}20 & 262 & 132956 & 2091 & 20\end{array}$

$\begin{array}{lllll}20 & 262 & 133008 & 2093 \quad 20\end{array}$

$20 \quad 262 \quad 133020 \quad 2095 \quad 20$

$-1$

$-2$

$-3$

$-4$

$-5$

$-6$

$-7$

$-8$

$-9$

$-10$

$-11$

$-12$

$-13$

$-14$ uncorr. corr. dif. uncorr. corr.

VERT.

$\begin{array}{lllllll}50245.5 & 50236.8 & 8.7 & 49403.2 & 49375.3 & 27.9 & -1380.8\end{array}$

$\begin{array}{llllllll}50764.1 & 50755.4 & 8.7 & 50396.2 & 50368.3 & 27.9 & -603.1\end{array}$

$\begin{array}{llllllll}51076.3 & 51067.6 & 8.7 & 50709.7 & 50681.9 & 27.8 & -601.0\end{array}$

$\begin{array}{llllllll}51449.3 & 51440.6 & 8.7 & 51078.7 & 51050.9 & 27.8 & -607.5\end{array}$

$\begin{array}{llllllll}51478.1 & 51469.4 & 8.7 & 51232.7 & 51204.9 & 27.8 & -402.3\end{array}$

$\begin{array}{lllllll}51367.1 & 51358.4 & 8.7 & 51192.2 & 51164.5 & 27.7 & -286.7\end{array}$

$\begin{array}{llllllll}51291.6 & 51282.9 & 8.7 & 51197.4 & 51169.7 & 27.7 & -154.4\end{array}$

$\begin{array}{llllllll}51275.3 & 51266.6 & 8.7 & 51197.2 & 51169.6 & 27.6 & -128.0\end{array}$

$\begin{array}{llllllll}51252.0 & 51243.4 & 8.6 & 51199.1 & 51171.5 & 27.6 & -86.7\end{array}$

$\begin{array}{lllllll}51225.1 & 51216.5 & 8.6 & 51171.8 & 51144.2 & 27.6 & -87.4\end{array}$

$\begin{array}{lllllll}51199.1 & 51190.5 & 8.6 & 51127.2 & 51099.7 & 27.5 & -117.9\end{array}$

$\begin{array}{llllllll}51189.6 & 51181.0 & 8.6 & 51087.6 & 51060.1 & 27.5 & -167.2\end{array}$

$\begin{array}{lllllll}51208.0 & 51199.4 & 8.6 & 51085.1 & 51057.7 & 27.4 & -201.5\end{array}$

$\begin{array}{llllllll}51243.0 & 51234.4 & 8.6 & 51118.1 & 51090.7 & 27.4 & -204.8\end{array}$

$\begin{array}{lllllll}51273.1 & 51264.5 & 8.6 & 51165.5 & 51138.2 & 27.3 & -176.4\end{array}$

$\begin{array}{llllllll}51298.8 & 51290.2 & 8.6 & 51225.0 & 51197.7 & 27.3 & -121.0\end{array}$

$\begin{array}{llllllll}51303.7 & 51295.2 & 8.5 & 51246.5 & 51219.3 & 27.2 & -93.8\end{array}$

$\begin{array}{llllllll}51304.5 & 51296.0 & 8.5 & 51250.3 & 51223.1 & 27.2 & -88.9\end{array}$

$\begin{array}{llllllll}51291.8 & 51283.3 & 8.5 & 51233.5 & 51206.3 & 27.2 & -95.6\end{array}$

$\begin{array}{llllllll}51262.9 & 51254.4 & 8.5 & 51189.6 & 51162.5 & 27.1 & -120.2\end{array}$

$\begin{array}{lllllll}51247.8 & 51239.3 & 8.5 & 51124.5 & 51097.4 & 27.1 & -202.1\end{array}$

$\begin{array}{lllllll}51260.9 & 51252.4 & 8.5 & 51070.9 & 51043.9 & 27.0 & -311.5\end{array}$

$\begin{array}{lllllll}51304.1 & 51295.6 & 8.5 & 51130.4 & 51103.4 & 27.0 & -284.8\end{array}$

$\begin{array}{llllllll}51331.4 & 51322.9 & 8.5 & 51193.2 & 51166.3 & 26.9 & -226.6\end{array}$

$\begin{array}{llllllll}51341.0 & 51332.5 & 8.5 & 51268.2 & 51241.3 & 26.9 & -119.3\end{array}$

$\begin{array}{llllllll}51318.9 & 51310.5 & 8.4 & 512828.2 & 51255.5 & 26.7 & -60.2\end{array}$

$\begin{array}{lllllll}51254.0 & 51245.6 & 8.4 & 51236.2 & 51209.6 & 26.6 & -29.2\end{array}$

$\begin{array}{llllllll}51136.1 & 51127.7 & 8.4 & 51115.1 & 51088.5 & 26.6 & -34.4\end{array}$

$\begin{array}{lllllll}50972.2 & 50963.8 & 8.4 & 508933.7 & 50867.1 & 26.6 & -128.7\end{array}$

$\begin{array}{llllllll}50774.4 & 50766.0 & 8.4 & 50644.1 & 50617.6 & 26.5 & -213.6\end{array}$

$\begin{array}{lllllll}50567.4 & 50559.1 & 8.3 & 503637.0 & 50340.5 & 26.5 & -328.5\end{array}$

$\begin{array}{llllllll}50271.3 & 50263.0 & 8.3 & 50015.5 & 49989.1 & 26.4 & -419.3\end{array}$

$\begin{array}{lllllll}49867.8 & 49859.5 & 8.3 & 49414.3 & 49387.9 & 26.4 & -743.4\end{array}$

$\begin{array}{llllllll}50597.6 & 50589.2 & 8.4 & 49437.1 & 49411.8 & 25.3\end{array}$

$\bullet$

-769.8
-138.1

$\begin{array}{llllllll}51164.3 & 51155.9 & 8.4 & 501318.8 & 50293.7 & 25.1 & -1386.1\end{array}$

$52141.4 \quad 52132.9 \quad 8.5$

$\begin{array}{llll}51491.4 & 51482.9 & 8.5\end{array}$

$51517.2 \quad 51508.7 \quad 8.5$
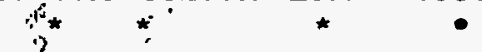

$51314.151305 .6 \quad 8.5$

$51256.4 \quad 51247.8 \quad 8.6$

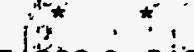

51282.951258 .024 .9

51 f 63.751138 .924 .8

$51188.0 \quad 51163.324 .7$

$\begin{array}{llllllll}51231.8 & 51223.2 & 8.6 & 51182.4 & 51157.8 & 24.6\end{array}$

$\begin{array}{lllllllll}51181.9 & 51173.3 & 8.6 & 51115.7 & 51091.1 & 24.6\end{array}$

$\begin{array}{llllllll}51119.1 & 51110.4 & 8.7 & 50994.2 & 50969.7 & 24.5\end{array}$

$\begin{array}{llllllll}51078.7 & 51070.0 & 8.7 & 50827.7 & 50803.3 & 24.4\end{array}$

$\begin{array}{lllllll}51166.5 & 51157.8 & 8.7 & 50747.6 & 50723.3 & 24.3\end{array}$

$\begin{array}{lllllll}51273.4 & 51264.7 & 8.7 & 50857.9 & 50833.6 & 24.3\end{array}$

51306.351297 .5

8.8

51065.151040 .924 .2

$-384.1$

$-246.6$

$-112.1$

$-81.0$

$-108.5$

$-204.8$

$-411.5$

$-686.7$

$-681.1$

$-395.4$

Page 34

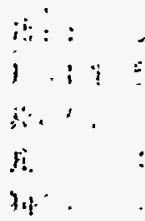


Hydroflouric Acid Spill Magnetic Survey Data

LINE DAY TIME STA. $X$

$20 \quad 262 \quad 133033 \quad 2097 \quad 20$

$20 \quad 262 \quad 133045 \quad 2099 \quad 20$

$20 \quad 262 \quad 133058 \quad 2101 \quad 20$

$\begin{array}{lllll}20 & 262 & 133112 & 2103 & 20\end{array}$

$20 \quad 262 \quad 133125 \quad 2105 \quad 20$

$\begin{array}{llllll}20 & 262 & 133138 & 2107 & 20\end{array}$

$20 \quad 262 \quad 133152 \quad 210920$

$20 \quad 262 \quad 133205 \quad 2111 \quad 20$

$20 \quad 262 \quad 133219 \quad 2113 \quad 20$

$\begin{array}{lllll}20 & 262 & 133231 & 2115 & 20\end{array}$

20

20

20

20

20

20

21

21

21

21

. 21

21

21

21

21

21

21

21

21

21

21

21

21

21

21

21

21

21

21

21

21

21

21

21

21

21

21

22

22 $\begin{array}{llll}262 & 133330 & 2117 & 20\end{array}$

$\begin{array}{llll}262 & 133343 & 2119 & 20\end{array}$

$\begin{array}{llll}262 & 133355 & 2121 & 20\end{array}$

$\begin{array}{llll}262 & 133408 & 2123 & 20\end{array}$

$\begin{array}{llll}262 & 133420 & 2125 & 20\end{array}$

$\begin{array}{llll}262 & 133432 & 2127 & 20\end{array}$

$\begin{array}{llll}262 & 133814 & 2141 & 21\end{array}$

$\begin{array}{llll}262 & 133826 & 2143 & 21\end{array}$

$\begin{array}{llll}262 & 133837 & 2145 & 21\end{array}$

$\begin{array}{llll}262 & 133849 & 2147 & 21\end{array}$

$\begin{array}{llll}262 & 133900 & 2149 & 21\end{array}$

$\begin{array}{llll}262 & 133913 & 2151 & 21\end{array}$

$\begin{array}{llll}262 & 133925 & 2153 & 21\end{array}$

$\begin{array}{llll}262 & 133937 & 2155 & 21\end{array}$

$\begin{array}{llll}262 & 133948 & 2157 & 21\end{array}$

$\begin{array}{llll}262 & 134000 & 2159 & 21\end{array}$

$\begin{array}{lllll}262 & 134013 & 2161 & 21\end{array}$

$\begin{array}{llll}262 & 134025 & 2163 & 21\end{array}$

$\begin{array}{llll}262 & 134037 & 2165 & 21\end{array}$

$\begin{array}{llll}262 & 134049 & 2167 & 21\end{array}$

$\begin{array}{llll}262 & 134101 & 2169 & 21\end{array}$

$\begin{array}{llll}262 & 134112 & 2171 & 21\end{array}$

$\begin{array}{llll}262 & 134124 & 2173 & 21\end{array}$

$\begin{array}{lllll}262 & 134136 & 2175 & 21\end{array}$

$\begin{array}{llll}262 & 134149 & 2177 & 21\end{array}$

$\begin{array}{lllll}262 & 134201 & 2179 & 21\end{array}$

$\begin{array}{llll}262 & 134217 & 2181 & 21\end{array}$

$\begin{array}{lllll}262 & 134229 & 2183 & 21\end{array}$

$\begin{array}{llll}262 & 134241 & 2185 & 21\end{array}$

$\begin{array}{lllll}262 & 134253 & 2187 & 21\end{array}$

$\begin{array}{llll}262 & 134304 & 2189 & 21\end{array}$

$\begin{array}{llll}262 & 134316 & 2191 & 21\end{array}$

$\begin{array}{llll}262 & 134415 & 2193 & 21\end{array}$

$\begin{array}{llll}262 & 134426 & 2195 & 21\end{array}$

$\begin{array}{lllll}262 & 134439 & 2197 & 21\end{array}$

$\begin{array}{llll}262 & 134450 & 2199 & 21\end{array}$

$\begin{array}{llll}262 & 134502 & 2201 & 21\end{array}$

$\begin{array}{llll}264 & 84005 & 10 & 22\end{array}$

$\begin{array}{llll}264 & 84014 & 12 & 22\end{array}$
$Y$

$-16$

$-17$

$-18$

$-19$

$-20$

$-21$

$-22$

$-23$

$-24$

$-25$

$-26$

$-27$

$-28$

$-29$

$-30$

$-31$

0

$-1$

$-2$

$-3$

$-4$

$-5$

$-6$

$-7$

$-8$

$-9$

$-10$

$-11$

$-12$

$-13$

$-14$

$-15$

$-16$

$-17$

$-18$

$-19$

$-20$

$-21$

$-22$

$-23$

$-24$

$-25$

$-26$

$-27$

$-28$

$-29$

$-30$

0

$-1$
TOP BOTTOM VERT.

uncorr. corr. dif. uncorr. corr. dif. GRAD.(nT/m)

$\begin{array}{lllllll}51326.3 & 51317.5 & 8.8 & 51216.1 & 51192.0 & 24.1 & -180.7\end{array}$

$\begin{array}{lllllll}51330.9 & 51322.1 & 8.8 & 51276.6 & 51252.6 & 24.0 & -89.0\end{array}$

$\begin{array}{llllllll}51331.7 & 51322.9 & 8.8 & 51284.4 & 51260.4 & 24.0 & -77.5\end{array}$

$\begin{array}{lllllll}51295.4 & 51286.5 & 8.9 & 51242.1 & 51218.2 & 23.9 & -87.4\end{array}$

$\begin{array}{llllllll}51237.9 & 51229.0 & 8.9 & 51133.0 & 51109.2 & 23.8 & -172.0\end{array}$

$\begin{array}{lllllll}51166.3 & 51157.4 & 8.9 & 50914.8 & 50891.1 & 23.7 & -412.3\end{array}$

$\begin{array}{lllllll}51249.1 & 51240.1 & 9.0 & 50612.4 & 50588.8 & 23.6 & -1043.8\end{array}$

$\begin{array}{llllllll}51429.3 & 51420.3 & 9.0 & 50616.5 & 50592.9 & 23.6 & -1332.5\end{array}$

$\begin{array}{llllllll}51442.9 & 51433.9 & 9.0 & 50983.3 & 50959.8 & 23.5 & -753.4\end{array}$

$\begin{array}{llllllll}51429.3 & 51420.3 & 9.0 & 51306.8 & 51283.4 & 23.4 & -200.8\end{array}$

$\begin{array}{llllllll}51413.3 & 51404.1 & 9.2 & 51373.0 & 51350.0 & 23.0 & -66.1\end{array}$

$\begin{array}{lllllll}51334.8 & 51325.6 & 9.2 & 51310.9 & 51287.9 & 23.0 & -39.2\end{array}$

$\begin{array}{llllllll}51172.6 & 51163.4 & 9.2 & 51133.0 & 51110.1 & 22.9 & -64.9\end{array}$

$\begin{array}{llllllll}50933.1 & 50923.8 & 9.3 & 50841.5 & 50818.7 & 22.8 & -150.2\end{array}$

$\begin{array}{llllllll}50542.8 & 50533.5 & 9.3 & 50351.7 & 50329.0 & 22.7 & -313.3\end{array}$

$\begin{array}{cccccccc}50023.150013 .8 & 9.3 & 49614.1 & 49591.4 & 22.7 & -670.5\end{array}$

$\begin{array}{lllllll}50825.8 & 50820.9 & 4.9 & 50069.2 & 50045.5 & 23.7 & -1240.3\end{array}$

$\begin{array}{llllllll}50809.2 & 50804.2 & 5.0 & 50294.7 & 50271.2 & 23.5 & -843.4\end{array}$

$\begin{array}{lllllll}51200.5 & 51195.3 & 5.2 & 51933.7 & 51910.5 & 23.2 & 1202.0\end{array}$

$52397.152391 .6 \quad 5.5$

*

$\begin{array}{lllllll}51421.1 & 51415.5 & 5.6 & 51003.2 & 51030.7 & 22.5\end{array}$

$\begin{array}{llllll}51242.8 & 51237.0 & 5.8 & 51112.2 & 51089.9 & 22.3\end{array}$

$\begin{array}{lllllll}51217.5 & 51211.6 & 5.9 & 51172.9 & 51150.9 & 22.0\end{array}$

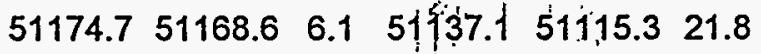

$\begin{array}{lllllll}51080.3 & 51074.1 & 6.2 & 50979.8 & 50958.3 & 21.5\end{array}$

$\begin{array}{lllllll}50934.0 & 50927.6 & 6.4 & 5066 & 6 & 50625.0 & 21.3\end{array}$

$\begin{array}{lllllll}50889.2 & 50882.7 & 6.5 & 49904.8 & 49883.7 & 21.1\end{array}$

$\begin{array}{lllllll}51604.0 & 51597.3 & 6.7 & 49639 & 39.5 & 49818.7 & 20.8\end{array}$

$\begin{array}{llllll}51333.4 & 51326.6 & 6.8 & 50510.4 & 50489.8 & 20.6\end{array}$

$\begin{array}{lllllll}51385.0 & 51378.0 & 7.0 & 509 \operatorname{g} 9.9 & 509079.5 & 20.4\end{array}$

$\begin{array}{lllllll}51370.4 & 51363.3 & 7.1 & 51242.8 & 51222.7 & 20.1\end{array}$

$\begin{array}{llllll}51375.2 & 51367.9 & 7.3 & 51344.9 & 51325.0 & 19.9\end{array}$

$\begin{array}{llllll}51354.9 & 51347.5 & 7.4 & 51.334 .2 & 51314.6 & 19.6\end{array}$

$\begin{array}{llllll}51284.0 & 51276.4 & 7.6 & 51243.9 & 51224.5 & 19.4\end{array}$

$\begin{array}{llllll}51123.2 & 51115.4 & 7.8 & 50931.9 & 50912.9 & 19.0\end{array}$

$\begin{array}{llllll}50897.5 & 50889.6 & 7.9 & 50155.0 & 50136.2 & 18.8\end{array}$

$52199.7 \quad 52191.6 \quad 8.1$

$52926.3 \quad 52918.1 \quad 8.2$

$51906.451898 .1 \quad 8.3$

$\begin{array}{lll}51616.2 & 51607.7 & 8.5\end{array}$

$51609.0 \quad 51599.8 \quad 9.2$

$51477.5 \quad 51468.1 \quad 9.4$

$51168.7 \quad 51159.2 \quad 9.5$

$50652.650642 .9 \quad 9.7$

$49600.7 \quad 49585.0 \quad 15.7$

$\begin{array}{lllllll}52069.0 & 52070.8 & -1.8 & 58598.0 & 58600.0 & -2.0\end{array}$

$\begin{array}{lllllll}50984.4 & 50986.2 & -1.8 & 49,571.8 & 49573.9 & -2.1\end{array}$

$49959.7 \quad 49941.4 \quad 18.3 \quad-4863.3$

$50 \dddot{914.7} 50896.6 \quad 18.1 \quad-1625.7$

$-315.2$

$-32.8$

14.4

$-57.0$

$-253.3$

$-531.0$

10703.3

$-2315.7$ 
Hydroflouric Acid Spill Magnetic Survey Data

TOP

-.. BOTTOM

uncorr. corr. dif. uncorr. corr.

VERT.

$\begin{array}{cccccc}\text { LINE } & \text { DAY } & \text { TIME } & \text { STA. } & \text { X } & \text { Y } \\ 22 & 264 & 84023 & 14 & 22 & -2 \\ 22 & 264 & 84032 & 16 & 22 & -3 \\ 22 & 264 & 84041 & 18 & 22 & -4 \\ 22 & 264 & 84050 & 20 & 22 & -5 \\ 22 & 264 & 84059 & 22 & 22 & -6 \\ 22 & 264 & 84108 & 24 & 22 & -7 \\ 22 & 264 & 84116 & 26 & 22 & -8 \\ 22 & 264 & 84124 & 28 & 22 & -9 \\ 22 & 264 & 84132 & 30 & 22 & -10 \\ 22 & 264 & 84142 & 32 & 22 & -11 \\ 22 & 264 & 84151 & 34 & 22 & -12 \\ 22 & 264 & 84159 & 36 & 22 & -13 \\ 22 & 264 & 84208 & 38 & 22 & -14 \\ 22 & 264 & 84216 & 40 & 22 & -15 \\ 22 & 264 & 84229 & 42 & 22 & -16 \\ 22 & 264 & 84239 & 44 & 22 & -17 \\ 22 & 264 & 84248 & 46 & 22 & -18 \\ 22 & 264 & 84255 & 48 & 22 & -19 \\ 22 & 264 & 84303 & 50 & 22 & -20 \\ 22 & 264 & 84313 & 52 & 22 & -21 \\ 22 & 264 & 84324 & 54 & 22 & -22 \\ 22 & 264 & 84332 & 56 & 22 & -23 \\ 22 & 264 & 84340 & 58 & 22 & -24 \\ 22 & 264 & 84348 & 60 & 22 & -25 \\ 22 & 264 & 84419 & 62 & 22 & -26 \\ 22 & 264 & 84427 & 64 & 22 & -27 \\ 22 & 264 & 84436 & 66 & 22 & -28 \\ 22 & 264 & 84444 & 68 & 22 & -29 \\ 22 & 264 & 84454 & 70 & 22 & -30 \\ 22 & 264 & 84501 & 72 & 22 & -31 \\ 23 & 264 & 84639 & 74 & 23 & -1 \\ 23 & 264 & 84648 & 76 & 23 & -2 \\ 23 & 264 & 84657 & 78 & 23 & -3 \\ 23 & 264 & 84704 & 80 & 23 & -4 \\ 23 & 264 & 84713 & 82 & 23 & -5 \\ 23 & 264 & 84722 & 84 & 23 & -6 \\ 23 & 264 & 84730 & 86 & 23 & -7 \\ 23 & 264 & 84738 & 88 & 23 & -8 \\ 23 & 264 & 84748 & 90 & 23 & -9 \\ 23 & 264 & 84756 & 92 & 23 & -10 \\ 23 & 264 & 84807 & 94 & 23 & -11 \\ 23 & 264 & 84816 & 96 & 23 & -12 \\ 23 & 264 & 84825 & 98 & 23 & -13 \\ 23 & 264 & 84832 & 100 & 23 & -14 \\ 23 & 264 & 84840 & 102 & 23 & -15 \\ 23 & 264 & 84912 & 104 & 23 & -16 \\ & 264 & 84926 & 106 & 23 & -17 \\ 234 & 84944 & 108 & 23 & -18 \\ 23 & 110 & 23 & -19 \\ 23 & & & & \end{array}$

$\begin{array}{llllll}50649.8 & 50651.6 & -1.8 & 50222.2 & 50224.4 & -2.2\end{array}$

dif. GRAD.(nT/m)

$50607.450609 .2-1.8 \quad 49886.8 \quad 49889.1-2.3$

$\begin{array}{llllllll}50997.4 & 50999.2 & -1.8 & 49615.6 & 49618.0 & -2.4 & -2265.2\end{array}$

$\begin{array}{llllllll}51234.8 & 51236.7 & -1.9 & 50404.0 & 50406.5 & -2.5 & -1362.0\end{array}$

$\begin{array}{llllllll}51068.2 & 51070.1 & -1.9 & 50683.8 & 50686.5 & -2.7 & -630.2\end{array}$

$\begin{array}{lllllll}51033.4 & 51035.3 & -1.9 & 50842.2 & 50845.0 & -2.8 & -313.4\end{array}$

$\begin{array}{lllllll}51057.4 & 51059.3 & -1.9 & 50975.2 & 50978.1 & -2.9 & -134.8\end{array}$

$\begin{array}{llllllll}51056.6 & 51058.6 & -2.0 & 51013.0 & 51016.0 & -3.0 & -71.5\end{array}$

$\begin{array}{llllllll}50960.4 & 50962.4 & -2.0 & 50873.0 & 50876.1 & -3.1 & -143.3\end{array}$

$\begin{array}{lllllll}50642.6 & 50644.6 & -2.0 & 50315.6 & 50318.8 & -3.2 & -536.1\end{array}$

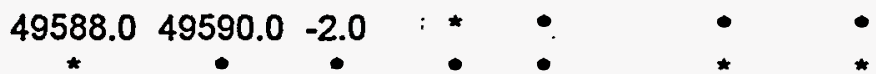

$-13$

$-14$

$-16$

$51545.651547 .7-2.150071 .450675 .1-3.7$

$\begin{array}{llllll}51545.6 & 51547.7 & -2.1 & 50671.4 & 50675.1 & -3.7\end{array}$

$-1433.1$

$\begin{array}{llllllll}51334.2 & 51336.3 & -2.1 & 51197.8 & 51201.6 & -3.8 & -223.6\end{array}$

$\begin{array}{lllllll}51361.2 & 51363.4 & -2.2 & 51375.2 & 51379.2 & -4.0 & 23.0\end{array}$

$\begin{array}{lllllll}51357.4 & 51359.6 & -2.2 & 51384.4 & 51388.5 & -4.1 & 44.3\end{array}$

$\begin{array}{llllllll}51294.2 & 51296.4 & -2.2 & 51295.0 & 51299.2 & -4.2 & 1.3\end{array}$

$\begin{array}{lllllll}51126.6 & 51128.8 & -2.2 & 51043.8 & 51048.1 & -4.3 & -135.7\end{array}$

$\begin{array}{llllllll}50507.4 & 50509.6 & -2.2 & 49892.8 & 49897.2 & -4.4 & -1007.5\end{array}$

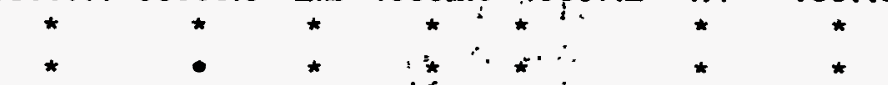

$\begin{array}{lllllll}52143.4 & 52145.7 & -2.3 & 50894.8 & 50899.6 & -4.8\end{array}$

$-2046.9$

$\begin{array}{llllllll}51886.6 & 51888.9 & -2.3 & 516 & 90.0 & 51694.9 & -4.9 & -322.3\end{array}$

$\begin{array}{llllllll}51908.0 & 51910.4 & -2.4 & 51940.4 & 51945.7 & -5.3 & 53.1\end{array}$

$\begin{array}{llllllll}51698.6 & 51701.0 & -2.4 & 51759.6 & 51765.0 & -5.4 & 100.0\end{array}$

$\begin{array}{lllllll}51203.6 & 51206.1 & -2.5 & 512113.4 & 51218.9 & -5.5 & 16.1\end{array}$

$\begin{array}{lllllll}50398.4 & 50400.9 & -2.5 & 5027.76 .6 & 50282.2 & -5.6 & -199.7\end{array}$

$\begin{array}{llllllll}49359.0 & 49361.5 & -2.5 & 48881.4 & 48887.1 & -5.7 & -782.9\end{array}$

$\begin{array}{lccccccc} & * & * & 45548.6 & 45554.4 & -5.8 & \\ 50613.6 & 50616.4 & -2.8 & 49514.0 & 49521.1 & -7.1 & -1802.6\end{array}$

$\begin{array}{llllllll}50223.8 & 50226.6 & -2.8 & 49743.0 & 49750.2 & -7.2 & -788.2\end{array}$

$\begin{array}{llllllll}50180.4 & 50183.2 & -2.8 & 49780.6 & 49787.9 & -7.3 & -655.4\end{array}$

$\begin{array}{llllllll}50405.0 & 50407.8 & -2.8 & 49946.6 & 49954.0 & -7.4 & -751.5\end{array}$

$\begin{array}{llllllll}50641.2 & 50644.1 & -2.9 & 50283.2 & 50290.7 & -7.5 & -586.9\end{array}$

$\begin{array}{lllllll}50763.8 & 50766.7 & -2.9 & 505508.2 & 50515.9 & -7.7 & -419.0\end{array}$

$\begin{array}{lllllll}50865.2 & 50868.1 & -2.9 & 50 \dot{1} 671.2 & 50679.0 & -7.8 & -318.0\end{array}$

$\begin{array}{llllllll}50917.8 & 50920.7 & -2.9 & 50815.0 & 50822.9 & -7.9 & -168.5\end{array}$

$\begin{array}{llllllll}50986.8 & 50989.8 & -3.0 & 50883.0 & 50891.0 & -8.0 & -170.2\end{array}$

$\begin{array}{llllllll}50852.0 & 50855.0 & -3.0 & 50 \dot{7} 00.6 & 50708.7 & -8.1 & -248.2\end{array}$

$\begin{array}{lllllll}50514.2 & 50517.2 & -3.0 & 500049.8 & 50058.0 & -8.2 & -761.3\end{array}$

$\begin{array}{ccccccc}* & * & \bullet & 48411.0 & 48419.4 & -8.4 & *\end{array}$

$\begin{array}{lllllll}51247.2 & 51250.3 & -3.1 & 50333.6 & 50342.2 & -8.6 & -1497.7\end{array}$

$\begin{array}{lllllll}51227.2 & 51230.3 & -3.1 & 50989.8 & 50998.5 & -8.7 & -389.2\end{array}$

$\begin{array}{lllllll}51285.0 & 51288.2 & -3.2 & 512366.4 & 51275.5 & -9.1 & -30.5\end{array}$

$\begin{array}{lllllll}51326.4 & 51329.6 & -3.2 & 51385.8 & 51395.1 & -9.3 & 97.4\end{array}$

$\begin{array}{lllllll}51319.8 & 51323.0 & -3.2 & 51363.0 & 51372.4 & -9.4 & 70.8\end{array}$

$\begin{array}{lllllll}51244.6 & 51247.9 & -3.3 & 51264.2 & 51273.7 & -9.5 & 32.1\end{array}$ 
TOP

$\begin{array}{cccccc}\text { LINE } & \text { DAY } & \text { TIME } & \text { STA. } & \text { X } & \text { Y } \\ 23 & 264 & 84951 & 112 & 23 & -20 \\ 23 & 264 & 85000 & 114 & 23 & -21 \\ 23 & 264 & 85014 & 116 & 23 & -22 \\ 23 & 264 & 85023 & 118 & 23 & -23 \\ 23 & 264 & 85030 & 120 & 23 & -24 \\ 23 & 264 & 85054 & 122 & 23 & -25 \\ 23 & 264 & 85106 & 124 & 23 & -26 \\ 23 & 264 & 85114 & 126 & 23 & -27 \\ 23 & 264 & 85124 & 128 & 23 & -28 \\ 24 & 264 & 85847 & 140 & 24 & -1 \\ 24 & 264 & 85859 & 142 & 24 & -2 \\ 24 & 264 & 85910 & 144 & 24 & -3 \\ 24 & 264 & 85920 & 146 & 24 & -4 \\ 24 & 264 & 85928 & 148 & 24 & -5 \\ 24 & 264 & 85937 & 150 & 24 & -6 \\ 24 & 264 & 85946 & 152 & 24 & -7 \\ 24 & 264 & 85954 & 154 & 24 & -8 \\ 24 & 264 & 90003 & 156 & 24 & -9 \\ 24 & 264 & 90011 & 158 & 24 & -10 \\ 24 & 264 & 90020 & 160 & 24 & -11 \\ 24 & 264 & 90029 & 162 & 24 & -12 \\ 24 & 264 & 90038 & 164 & 24 & -13 \\ 24 & 264 & 90048 & 166 & 24 & -14 \\ 24 & 264 & 90056 & 168 & 24 & -15 \\ 24 & 264 & 90103 & 170 & 24 & -16 \\ 24 & 264 & 90112 & 172 & 24 & -17 \\ 24 & 264 & 90120 & 174 & 24 & -18 \\ 24 & 264 & 90128 & 176 & 24 & -19 \\ 24 & 264 & 90135 & 178 & 24 & -20 \\ 24 & 264 & 90143 & 180 & 24 & -21 \\ 24 & 264 & 90152 & 182 & 24 & -22 \\ 24 & 264 & 90202 & 184 & 24 & -23 \\ 24 & 264 & 90210 & 186 & 24 & -24 \\ 24 & 264 & 90218 & 188 & 24 & -25 \\ 24 & 264 & 90239 & 190 & 24 & -26 \\ 24 & 264 & 90251 & 192 & 24 & -27 \\ 24 & 264 & 90300 & 194 & 24 & -28 \\ 25 & 264 & 90626 & 196 & 25 & -4 \\ 25 & 264 & 90634 & 198 & 25 & -5 \\ 25 & 264 & 90642 & 200 & 25 & -6 \\ 25 & 264 & 90651 & 202 & 25 & -7 \\ 25 & 264 & 90700 & 204 & 25 & -8 \\ 25 & 264 & 90708 & 206 & 25 & -9 \\ 25 & 264 & 90716 & 208 & 25 & -10 \\ 25 & 264 & 90724 & 210 & 25 & -11 \\ 25 & 264 & 90734 & 212 & 25 & -12 \\ 25 & 264 & 90742 & 214 & 25 & -13 \\ 25 & 264 & 90751 & 216 & 25 & -14 \\ 25 & 264 & 90759 & 218 & 25 & -15 \\ & & & & & \end{array}$

uncorr. corr. dif. uncorr. corr.

$51030.4 \quad 51033.7 \quad-3.3 \quad 50941.6 \quad 50951.2 \quad-9.6$

$\begin{array}{lllllll}50706.4 & 50709.7 & -3.3 & 50111.6 & 50121.3 & -9.7\end{array}$

$\begin{array}{lllllll}51090.8 & 51094.1 & -3.3 & 49726.6 & 49736.5 & -9.9\end{array}$

$\begin{array}{llllll}52003.6 & 52007.0 & -3.4 & 51085.4 & 51095.4 & -10.0\end{array}$

$\begin{array}{lllllll}52431.6 & 52435.0 & -3.4 & 51907.8 & 51917.9 & -10.1\end{array}$

$\begin{array}{lllllll}53523.0 & 53526.4 & -3.4 & 53257.4 & 53267.8 & -10.4\end{array}$

$\begin{array}{lllllll}53767.8 & 53771.3 & -3.5 & 54011.8 & 54022.4 & -10.6\end{array}$

$\begin{array}{lllllll}52198.4 & 52201.9 & -3.5 & 52469.2 & 52479.9 & -10.7\end{array}$

$58536.858547 .6-10.8$

$\begin{array}{llllll}50895.6 & 50897.9 & -2.3 & 58594.2 & 58602.5 & -8.3\end{array}$

$\begin{array}{llllllll}50074.4 & 50076.7 & -2.3 & 48995.8 & 49004.0 & -8.2\end{array}$

$\begin{array}{lllllll}49477.6 & 49479.9 & -2.3 & 48873.2 & 48881.3 & -8.1\end{array}$

$\begin{array}{lllllll}49890.0 & 49892.2 & -2.2 & 49132.4 & 49140.5 & -8.1\end{array}$

$\begin{array}{lllllll}50248.2 & 50250.4 & -2.2 & 49675.2 & 49683.3 & -8.1\end{array}$

$\begin{array}{lllllll}50542.4 & 50544.6 & -2.2 & 50183.4 & 50191.4 & -8.0\end{array}$

$\begin{array}{llllllll}50743.8 & 50746.0 & -2.2 & 50488.8 & 50496.8 & -8.0\end{array}$

$\begin{array}{llllllll}50859.0 & 50861.2 & -2.2 & 50670.8 & 50678.7 & -7.9\end{array}$

$\begin{array}{lllllll}50933.2 & 50935.3 & -2.1 & 50783.6 & 50791.5 & -7.9\end{array}$

$\begin{array}{llllllll}50917.8 & 50919.9 & -2.1 & 50760.2 & 50768.0 & -7.8\end{array}$

$\begin{array}{lllllll}50748.8 & 50750.9 & -2.1 & 50532.6 & 50540.4 & -7.8\end{array}$

$\begin{array}{lllllll}50562.4 & 50564.5 & -2.1 & 50154.6 & 50162.3 & -7.7\end{array}$

$\begin{array}{llllllll}50849.4 & 50851.4 & -2.0 & 50060.4 & 50068.1 & -7.7\end{array}$

$\begin{array}{lllllll}50941.6 & 50943.6 & -2.0 & 50511.8 & 50519.4 & -7.6\end{array}$

$\begin{array}{lllllll}51068.6 & 51070.6 & -2.0 & 50806.6 & 50814.2 & -7.6\end{array}$

$\begin{array}{lllllll}51182.2 & 51184.2 & -2.0 & 51078.6 & 51086.2 & -7.6\end{array}$

$\begin{array}{lllllll}51260.0 & 51261.9 & -1.9 & 51233.2 & 51240.7 & -7.5\end{array}$

$\begin{array}{lllllll}51289.2 & 51291.1 & -1.9 & 51269.0 & 51276.5 & -7.5\end{array}$

$\begin{array}{lllllll}51292.0 & 51293.9 & -1.9 & 512272.4 & 51279.8 & -7.4\end{array}$

$\begin{array}{lllllll}51258.8 & 51260.7 & -1.9 & 51,240.8 & 51248.2 & -7.4\end{array}$

$\begin{array}{lllllll}51248.8 & 51250.6 & -1.8 & 51124.0 & 51131.4 & -7.4\end{array}$

$\begin{array}{lllllll}51471.0 & 51472.8 & -1.8 & 51 & 88.6 & 51195.9 & -7.3\end{array}$

$\begin{array}{lllllll}51973.6 & 51975.4 & -1.8 & 51491.6 & 51498.9 & -7.3\end{array}$

$\begin{array}{lllllll}52860.0 & 52861.8 & -1.8 & 52371.8 & 52379.0 & -7.2\end{array}$

$\begin{array}{lllllll}58625.2 & 58627.0 & -1.8 & 58651.6 & 58658.8 & -7.2\end{array}$

$\begin{array}{llllllll}58603.0 & 58604.7 & -1.7 & 58637.4 & 58644.5 & -7.1\end{array}$

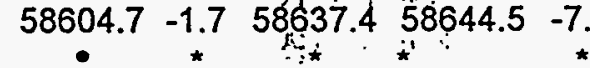

$\begin{array}{lllllll}52010.8 & 52011.9 & -1.1 & 58590.8 & 58596.7 & -5.9\end{array}$ $\begin{array}{llllll}58620.6 & 58621.6 & -1.0 & 58614.4 & 586320.3 & -5.9\end{array}$

$\begin{array}{llllllll}49630.6 & 49631.6 & -1.0 & 47661.2 & 47667.1 & -5.9\end{array}$

$\begin{array}{lllllll}50132.2 & 50133.2 & -1.0 & 49447.6 & 49453.4 & -5.8\end{array}$

$\begin{array}{lllllll}50458.0 & 50459.0 & -1.0 & 500087.0 & 50092.8 & -5.8\end{array}$

$\begin{array}{lllllll}50748.8 & 50749.8 & -1.0 & 50446.2 & 50451.9 & -5.7\end{array}$

$\begin{array}{lllllll}50957.4 & 50958.3 & -0.9 & 50 ́ 728.8 & 50734.5 & -5.7\end{array}$

$\begin{array}{lllllll}51015.4 & 51016.3 & -0.9 & 50821.0 & 50826.7 & -5.7\end{array}$

$\begin{array}{llllll}50978.4 & 50979.3 & -0.9 & 507790.4 & 50796.0 & -5.6\end{array}$

$\begin{array}{lllllll}50924.6 & 50925.5 & -0.9 & 50716.6 & 50722.2 & -5.6\end{array}$

$\begin{array}{llllll}50935.6 & 50936.4 & -0.8 & 507720.2 & 50725.7 & -5.5\end{array}$

$\begin{array}{llllll}50996.6 & 50997.4 & -0.8 & 50 \zeta 85.2 & 50790.7 & -5.5\end{array}$
VERT.

GRAD.(nT/m)

$-145.6$

$-975.1$

$-2236.4$

$-1505.2$

$-858.7$

$-435.4$

400.0

443.9

12620.7

$-1768.2$

$-990.8$

$-1242.0$

$-939.3$

$-588.5$

$-418.0$

$-308.5$

$-245.2$

$-258.4$

$-354.4$

$-668.5$

$-1293.4$

$-704.6$

$-429.5$

$-169.8$

$-43.9$

$-33.1$

$-32.1$

$-29.5$

$-204.6$

$-462.9$

$-790.2$

$-800.3$

43.3

56.4

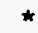

10786.9

$-10.2$

$-3228.5$

$-1122.3$

$-608.2$

$-496.1$

$-374.8$

$-318.7$

$-308.2$

$-341.0$

$-353.1$

$-346.6$ 
LINE DAY TIME STA. $X \quad Y$ 25264

$25 \quad 264$

$25 \quad 264$

$25 \quad 264$

$25 \quad 264$

$25 \quad 264$

25264

$25 \quad 264$

$25 \quad 264$

$25 \quad 264$

$25 \quad 264$

$25 \quad 264$

26

26

26

26

i. 26

26

26

.26

' 26

26

26

26

26

26

26

26

26

26

26

26

26

26

27

27

27

27

27

27

27

$27 \quad 264$

$27 \quad 264$

$27 \quad 264$

$27 \quad 264$

$27 \quad 264$

$27 \quad 264$

$27 \quad 264$ $\begin{array}{llll}90808 & 220 & 25 & -16\end{array}$

$\begin{array}{llll}90815 & 222 & 25 & -17\end{array}$

$\begin{array}{llll}90822 & 224 & 25 & -18\end{array}$

$\begin{array}{llll}90833 & 226 & 25 & -19\end{array}$

$\begin{array}{llll}90841 & 228 & 25 & -20\end{array}$

$\begin{array}{llll}90850 & 230 & 25 & -21\end{array}$

$\begin{array}{llll}90858 & 232 & 25 & -22\end{array}$

$\begin{array}{llll}90907 & 234 & 25 & -23\end{array}$

$\begin{array}{llll}90950 & 236 & 25 & -24\end{array}$

$\begin{array}{llll}90959 & 238 & 25 & -25\end{array}$

$\begin{array}{llll}91023 & 240 & 25 & -26\end{array}$

$\begin{array}{llll}91031 & 242 & 25 & -27\end{array}$

$\begin{array}{llll}91042 & 244 & 25 & -28\end{array}$

$\begin{array}{llll}91518 & 246 \quad 26\end{array}$

$91528248 \quad 26$

$91536 \quad 25026$

$91544 \quad 252 \quad 26$

$91554 \quad 254 \quad 26$

91603

91612

91621

91629

91636

91647

91658

91705

91714

91722

91730

91738

91746

91754

91802

91810

91818

92103

92111

92120

92128

92136

92143

92150

92157

92204

92213

92221

92228

92235

92244
25626

25826

26226

26426

26626

26826

27026

27226

27426

27626

27826

28026

$\begin{array}{lll}282 & 26 & -23.5\end{array}$

$\begin{array}{lll}284 & 26 & -24.5\end{array}$

$\begin{array}{lll}286 & 26 & -25.5\end{array}$

$\begin{array}{lll}288 & 26 & -26.5\end{array}$

$\begin{array}{lll}290 & 27 & -6.5\end{array}$

$\begin{array}{lll}292 & 27 & -7.5\end{array}$

$\begin{array}{lll}294 & 27 & -8.5\end{array}$

$\begin{array}{lll}296 & 27 & -9.5\end{array}$

$\begin{array}{lll}298 & 27 & -10.5\end{array}$

$\begin{array}{llll}300 & 27 & -11.5\end{array}$

$\begin{array}{lll}302 & 27 & -12.5\end{array}$

$\begin{array}{lll}304 & 27 & -13.5\end{array}$

$\begin{array}{llll}306 & 27 & -14.5\end{array}$

$\begin{array}{llll}308 & 27 & -15.5\end{array}$

$\begin{array}{llll}310 & 27 & -16.5\end{array}$

$\begin{array}{llll}312 & 27 & -17.5\end{array}$

$\begin{array}{llll}314 & 27 & -18.5\end{array}$

$\begin{array}{lll}316 & 27 & -19.5\end{array}$
26026
TOP

BOTTOM

uncorr. corr. dif. uncorr. corr.

$\begin{array}{llllll}51039.0 & 51039.8 & -0.8 & 50842.2 & 50847.6 & -5.4\end{array}$

$\begin{array}{lllllll}51080.2 & 51081.0 & -0.8 & 50885.4 & 50890.8 & -5.4\end{array}$

$\begin{array}{lllllll}51157.8 & 51158.5 & -0.7 & 50948.4 & 50953.8 & -5.4\end{array}$

$\begin{array}{lllllll}51228.2 & 51228.9 & -0.7 & 51017.4 & 51022.7 & -5.3\end{array}$

$\begin{array}{lllllll}51287.8 & 51288.5 & -0.7 & 51122.6 & 51127.9 & -5.3\end{array}$

$\begin{array}{llllllll}51392.0 & 51392.7 & -0.7 & 51172.2 & 51177.4 & -5.2\end{array}$

$\begin{array}{lllllll}51410.4 & 51411.1 & -0.7 & 51241.6 & 51246.8 & -5.2\end{array}$

$\begin{array}{lllllll}52568.0 & 52568.6 & -0.6 & 51666.4 & 51671.5 & -5.1\end{array}$

$\begin{array}{lllllll}52821.4 & 52821.9 & -0.5 & 52184.8 & 52189.7 & -4.9\end{array}$

$\begin{array}{lllllll}58628.4 & 58628.9 & -0.5 & 53928.8 & 53933.7 & -4.9\end{array}$

$\begin{array}{lllllll}58613.6 & 58614.0 & -0.4 & 58582.6 & 58587.3 & -4.7\end{array}$

$\begin{array}{lllllll}58643.2 & 58643.6 & -0.4 & 58661.2 & 58665.9 & -4.7\end{array}$

$\begin{array}{lllllll}58595.0 & 58595.4 & -0.4 & 58560.0 & 58564.6 & -4.6\end{array}$

$\begin{array}{cccccc}* & * & * & \bullet & * & 1 \\ * & * & 47548.4 & 47551.6 & -3.2\end{array}$

$\begin{array}{lllllll}49652.0 & 49651.6 & 0.4 & 48545.2 & 48548.4 & -3.2\end{array}$

$\begin{array}{llllllll}50420.8 & 50420.3 & 0.5 & 49636.6 & 49639.7 & -3.1\end{array}$

$\begin{array}{lllllll}50963.6 & 50963.1 & 0.5 & 50452.2 & 50455.3 & -3.1\end{array}$

$\begin{array}{llllllll}51136.6 & 51136.1 & 0.5 & 50794.6 & 50797.6 & -3.0\end{array}$

$\begin{array}{lllllll}51109.4 & 51108.9 & 0.5 & 50862.6 & 50865.6 & -3.0\end{array}$

$\begin{array}{lllllll}51053.4 & 51052.8 & 0.6 & 50875.0 & 50877.9 & -2.9\end{array}$

$\begin{array}{llllllll}51000.4 & 50999.8 & 0.6 & 50836.8 & 50839.7 & -2.9\end{array}$

$\begin{array}{lllllll}50958.8 & 50958.2 & 0.6 & 50808.2 & 50811.1 & -2.9\end{array}$

$\begin{array}{lllllll}50942.4 & 50941.8 & 0.6 & 50746.2 & 50749.0 & -2.8\end{array}$

$\begin{array}{lllllll}50948.6 & 50947.9 & 0.7 & 50599.2 & 50602.0 & -2.8\end{array}$

$\begin{array}{lllllll}51138.8 & 51138.1 & 0.7 & 50628.8 & 50631.5 & -2.7\end{array}$

$\begin{array}{lllllll}51296.2 & 51295.5 & 0.7 & 50632.8 & 50635.5 & -2.7\end{array}$

$\begin{array}{llllllll}51242.2 & 51241.5 & 0.7 & 50793.8 & 50796.4 & -2.6\end{array}$

$\begin{array}{lllllll}51213.6 & 51212.8 & 0.8 & 50928.0 & 50930.6 & -2.6\end{array}$

$\begin{array}{llllllll}51328.6 & 51327.8 & 0.8 & 51019.4 & 51021.9 & -2.5\end{array}$

$\begin{array}{lllllll}51743.4 & 51742.6 & 0.8 & 51217.8 & 51220.3 & -2.5\end{array}$

$\begin{array}{lllllll}52967.0 & 52966.2 & 0.8 & 518 & 18.4 & 51875.9 & -2.5\end{array}$

$\begin{array}{llllllll}58612.6 & 58611.7 & 0.9 & 586119.4 & 58621.8 & -2.4\end{array}$

$\begin{array}{lllllll}58625.6 & 58624.7 & 0.9 & 58604.2 & 58606.6 & -2.4\end{array}$

$\begin{array}{llllllll}58594.6 & 58593.7 & 0.9 & 5864 & 41.0 & 58643.4 & -2.4\end{array}$

$\begin{array}{llllllll}58589.6 & 58588.3 & 1.3 & 58549.6 & 58551.1 & -1.5\end{array}$

$\begin{array}{lllllll}52122.6 & 52121.2 & 1.4 & 58636.0 & 58637.5 & -1.5\end{array}$

$\begin{array}{lllllll}58562.2 & 58560.8 & 1.4 & 58556.8 & 58558.2 & -1.4\end{array}$

$\begin{array}{lllllll}51379.6 & 51378.2 & 1.4 & 51542.4 & 51543.8 & -1.4\end{array}$

$\begin{array}{lllllll}51473.4 & 51472.0 & 1.4 & 507.82 .2 & 50783.6 & -1.4\end{array}$

$\begin{array}{lllllll}51192.2 & 51190.7 & 1.5 & 50785.2 & 50786.5 & -1.3\end{array}$

$\begin{array}{lllllll}51028.0 & 51026.5 & 1.5 & 508066.2 & 50807.5 & -1.3\end{array}$

$\begin{array}{lllllll}51079.0 & 51077.5 & 1.5 & 50803.2 & 50804.4 & -1.2\end{array}$

$\begin{array}{lllllll}50797.0 & 50795.5 & 1.5 & 50897.2 & 50898.4 & -1.2\end{array}$

$\begin{array}{lll}50741.2 & 50739.7 & 1.5\end{array}$

$50 \hat{5} 14.0 \quad 50515.2-1.2$

$50670.450668 .8 \quad 1.6$

$\begin{array}{llll}49989.6 & 49990.7 & -1.1\end{array}$

$54282.854281 .2 \quad 1.6$

$52116.252114 .6 \quad 1.6$

$586024.458625 .5-1.1$

$58558.258559 .3-1.1$

$51230.0 \quad 51228.4 \quad 1.6$

$50364.6 \quad 50365.6 \quad-1.0$
VERT.

RAD.(nT/m)

$-322.6$

$-319.3$

$-343.3$

$-345.6$

$-270.8$

$-360.3$

$-276.7$

$-1478.0$

$-1043.6$

$-7704.3$

$-50.8$

29.5

$-57.4$

a

$-1814.4$

$-1285.6$

$-838.4$

$-560.7$

$-404.6$

$-292.5$

$-268.2$

$-246.9$

$-321.6$

$-572.8$

$-836.1$

$-1087.5$

$-735.1$

$-468.2$

$-506.9$

$-861.6$

$-1792.8$

11.1

$-35.1$

76.1

$-65.6$

10677.7

$-8.9$

266.9

$-1133.1$

$-667.2$

$-363.6$

$-452.1$

164.3

$-372.5$

$-1116.1$

7117.4

10560.7

$-1418.7$ 
Hydroflouric Acid Spill Magnetic Survey Data

TOP

LINE DAY TIME STA. $X \quad Y$ uncorr. corr.

$\begin{array}{lll}27 & 264 & 92251\end{array}$

$\begin{array}{lll}27 & 264 & 92300\end{array}$

$\begin{array}{lll}27 & 264 & 92308\end{array}$

$\begin{array}{lll}27 & 264 & 92316\end{array}$

$\begin{array}{lll}27 & 264 & 92324\end{array}$

$\begin{array}{lll}27 & 264 & 92332\end{array}$

$\begin{array}{lll}28 & 264 & 92602\end{array}$

$\begin{array}{lll}28 & 264 & 92614\end{array}$

$\begin{array}{lll}28 & 264 & 92624\end{array}$

$\begin{array}{lll}28 & 264 & 92633\end{array}$

$\begin{array}{lll}28 & 264 & 92642\end{array}$

$\begin{array}{lll}28 & 264 & 92651\end{array}$

$\begin{array}{lll}28 & 264 & 92659\end{array}$

$\begin{array}{lll}28 & 264 & 92709\end{array}$

$\begin{array}{lll}28 & 264 & 92718\end{array}$

$\begin{array}{lll}28 & 264 & 92726\end{array}$

$\begin{array}{lll}28 & 264 & 92734\end{array}$

$\begin{array}{lll}28 & 264 & 92745\end{array}$

$\begin{array}{lll}28 & 264 & 92754\end{array}$

$\begin{array}{lll}28 & 264 & 92802\end{array}$

$\begin{array}{lll}28 & 264 & 92810\end{array}$

$\begin{array}{lll}28 & 264 & 92818\end{array}$

$\begin{array}{lll}28 & 264 & 92826\end{array}$

$\begin{array}{lll}29 & 264 & 93038\end{array}$

$\begin{array}{lll}29 & 264 & 93046\end{array}$

$\begin{array}{lll}29 & 264 & 93053\end{array}$

$\begin{array}{lll}29 & 264 & 93101\end{array}$

$\begin{array}{lll}29 & 264 & 93108\end{array}$

$\begin{array}{lll}29 & 264 & 93116\end{array}$

$\begin{array}{lll}29 & 264 & 93124\end{array}$

$\begin{array}{lll}29 & 264 & 93132\end{array}$

$\begin{array}{lll}29 & 264 & 93140\end{array}$

$\begin{array}{lll}29 & 264 & 93147\end{array}$

$\begin{array}{lll}29 & 264 & 93155\end{array}$

$\begin{array}{lll}29 & 264 & 93202\end{array}$

$\begin{array}{lll}29 & 264 & 93210\end{array}$

$\begin{array}{lll}29 & 264 & 93217\end{array}$

$\begin{array}{lll}30 & 264 & 93234\end{array}$

$\begin{array}{lll}30 & 264 & 93241\end{array}$

$\begin{array}{lll}30 & 264 & 93249\end{array}$

$\begin{array}{lll}31 & 264 & 93349\end{array}$

$\begin{array}{lll}31 & 264 & 93357\end{array}$

$\begin{array}{lll}31 & 264 & 93404\end{array}$

$\begin{array}{lll}318 & 27 & -20.5\end{array}$

$\begin{array}{lll}320 & 27 & -21.5\end{array}$

$\begin{array}{lll}322 & 27 & -22.5\end{array}$

$\begin{array}{llll}324 & 27 & -23.5\end{array}$

$\begin{array}{llll}326 & 27 & -24.5\end{array}$

$\begin{array}{llll}328 & 27 & -25.5\end{array}$

$\begin{array}{lll}330 & 28 & -8.25\end{array}$

$\begin{array}{lll}332 & 28 & -9.25\end{array}$

$\begin{array}{lll}334 & 28 & -10.25\end{array}$

$\begin{array}{lll}336 & 28 & -11.25\end{array}$

$\begin{array}{llll}338 & 28 & -12.25\end{array}$

$\begin{array}{lll}340 & 28 & -13.25\end{array}$

$\begin{array}{lll}342 & 28 & -14.25\end{array}$

$\begin{array}{llll}344 & 28 & -15.25 & 508\end{array}$

$\begin{array}{lll}346 & 28 & -16.25\end{array}$

$\begin{array}{lll}348 & 28 & -17.25\end{array}$

$\begin{array}{lll}350 & 28 & -18.25\end{array}$

$\begin{array}{lll}352 & 28 & -19.25\end{array}$

$\begin{array}{llll}354 & 28 & -20.25 & 501\end{array}$

$\begin{array}{llll}356 & 28 & -21.25 & 493\end{array}$

$368 \quad 29$

$\begin{array}{ll}370 & 29\end{array}$

$-12$

$-13$

37229

$374 \quad 29$

$-14$

$-15$

$376 \quad 29$

$\begin{array}{ll}378 & 29\end{array}$

$\begin{array}{ll}380 & 29\end{array}$

38229

38429

$\begin{array}{ll}386 & 29\end{array}$

$\begin{array}{ll}388 & 29\end{array}$

$390 \quad 29$

39230

39431

$396 \quad 32$

$398 \quad 30$

$400 \quad 31$

40232
A. $X \quad Y$ uncorr. corr. dif. uncorr. corr.

$49379.6 \quad 49377.1 \quad 2.5$

$\begin{array}{lllllll}358 & 28 & -22.25 & 42393.6 & 42391.1 & 2.5\end{array}$

$\begin{array}{llllll}360 & 28 & -23.25 & 52356.2 & 52353.7 & 2.5\end{array}$

$\begin{array}{lllllll}362 & 28 & -24.25 & 58618.4 & 58615.8 & 2.6\end{array}$

$\begin{array}{lllllll}364 & 29 & -10 & 58684.4 & 58681.5 & 2.9\end{array}$

$\begin{array}{lllllll}366 & 29 & -11 & 52171.2 & 52168.2 & 3.0\end{array}$
$-16$

$-17$

$-18$

$-19$

$-20$

$-21$

$-22$

$-23$

$-15$

$-15$

$-15$

$-19$

$-19$

$-19$
$50884.0 \quad 50881.0 \quad 3.0$

$50717.250714 .2 \quad 3.0$

$50644.450641 .4 \quad 3.0$

$50516.450513 .4 \quad 3.0$

50295.250292 .13 .1

$50094.8 \quad 50091.7 \quad 3.1$

$\begin{array}{llll}51514.0 & 51510.9 & 3.1\end{array}$

$\begin{array}{llll}50716.8 & 50713.7 & 3.1\end{array}$

$\begin{array}{llll}49515.0 & 49511.9 & 3.1\end{array}$

$52195.252192 .0 \quad 3.2$

$58600.258597 .0 \quad 3.2$

$58604.258601 .0 \quad 3.2$

$50080.050076 .7 \quad 3.3$

$52089.452086 .1 \quad 3.3$

$58594.2 \quad 58590.9 \quad 3.3$

$\begin{array}{llll}48401.6 \quad 48398.1 & 3.5\end{array}$

$\begin{array}{llll}58674.4 & 58670.9 & 3.5\end{array}$

$\begin{array}{lll}58620.2 & 58616.7 & 3.5\end{array}$
$50129.450130 .4 \quad-1$.

$\begin{array}{llll}49955.0 & 49955.9 & -0.9\end{array}$

$\begin{array}{llll}49527.0 & 49527.9 & -0.9\end{array}$

$\begin{array}{llll}58608.8 & 58609.6 & -0.8\end{array}$

$\begin{array}{llll}58612.2 & 58613.0 & -0.8\end{array}$

$\begin{array}{llll}58609.2 & 58610.0 & -0.8\end{array}$

$\begin{array}{llll}58587.6 & 58587.6 & 0.0\end{array}$

$\begin{array}{lll}58592.2 & 58592.2 & 0.0\end{array}$

$\begin{array}{llll}50504.8 & 50504.7 & 0.1\end{array}$

$\begin{array}{lll}50581.2 & 50581.1 & 0.1\end{array}$

$\begin{array}{lll}50648.6 & 50648.4 & 0.2\end{array}$

$\begin{array}{lll}50679.6 & 50679.4 & 0.2\end{array}$

$\begin{array}{lll}50631.4 & 50631.1 & 0.3\end{array}$

$\begin{array}{llll}50459.4 & 50459.1 & 0.3\end{array}$

$\begin{array}{llll}49933.4 & 49933.0 & 0.4\end{array}$

$\begin{array}{lll}58571.2 & 58570.8 & 0.4\end{array}$

$\begin{array}{lll}58579.0 & 58578.5 & 0.5\end{array}$

$\begin{array}{lll}58604.6 & 58604.1 & 0.5\end{array}$

$\begin{array}{llll}49356.2 & 49355.6 & 0.6\end{array}$

$\begin{array}{llll}48601.8 & 48601.2 & 0.6\end{array}$

$58592.6 \quad 58592.0 \quad 0.6$

(3)

$58551.4 \quad 58550.7 \quad 0.7$

58606.658605 .21 .4

$\begin{array}{llll}49671.8 & 49670.4 & 1.4\end{array}$

$\begin{array}{lll}5000 \% 33.8 & 50082.3 & 1.5\end{array}$

$\begin{array}{llll}50285.0 & 50283.5 & 1.5\end{array}$

$50373.0 \quad 50371.5 \quad 1.5$

$50293.4 \quad 50291.8 \quad 1.6$

$50020.8 \quad 50019.2 \quad 1.6$

$4992.8 \quad 49191.1 \quad 1.7$

$58597.6 \quad 58595.9 \quad 1.7$

$\begin{array}{llll}52219.8 & 52218.1 & 1.7\end{array}$

$\begin{array}{llll}48583.8 & 48582.0 & 1.8\end{array}$

$\begin{array}{llll}47.3117 .6 & 47315.8 & 1.8\end{array}$

$58605.658603 .8 \quad 1.8$

$58639.4 \quad 58637.5 \quad 1.9$

$49705.8 \quad 49703.8 \quad 2.0$

$\begin{array}{llll}58539.0 & 58537.0 & 2.0\end{array}$

$58648.8 \quad 58646.8 \quad 2.0$

$47.940 .4 \quad 47938.12 .3$

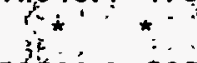

$58563.8 \quad 58561.4 \quad 2.4$
VERT.

GRAD.(nT/m)

$-876.4$

$-986.6$

$-3899.0$

10504.9

$-25.6$

42.6

$-95.4$

60494.8

$-3844.3$

$-1647.2$

$-693.1$

$-383.6$

$-266.2$

$-309.5$

$-882.0$

12666.9

9638.0

9717.7

$-1368.9$

$-1275.1$

26555.7

09.8

$-127.5$

$-4097.4$

$-1311.8$

$-708.5$

$-444.9$

$-365.6$

$-449.8$

$-1478.7$

11612.5

2463.9

$-1526.6$

$-7996.1$

8.9

57.7

$-613.4$

10573.1

89.5

$-756.1$

$-92.5$ 
Hydroflouric Acid Spill Magnetic Survey Coordinate Transformatiom'

Grid to Site Coordinate Transformation

\begin{tabular}{|c|c|c|c|c|c|c|c|}
\hline & D & SI & TE & \multicolumn{2}{|c|}{ GRID } & \multicolumn{2}{|c|}{ SITE } \\
\hline$x$ & $Y$ & EASTING & NORTHING & $x$ & $Y$ & EASTING & NORTHING \\
\hline 0 & 0 & 50127.11 & 61821.69 & 11 & -25 & 50163.19 & 61739.69 \\
\hline 0 & -1 & 50127.11 & 61818.41 & 11 & -26 & 50163.19 & 61736.41 \\
\hline 0 & -2 & 50127.11 & 61815.13 & 11 & -27 & 50163.19 & 61733.13 \\
\hline 0 & -3 & 50127.11 & 61811.85 & 11 & -28 & 50163.19 & 61729.85 \\
\hline 0 & -4 & 50127.11 & 61808.57 & 11 & -29 & 50163.19 & 61726.57 \\
\hline 0 & -5 & 50127.11 & 61805.29 & 11 & -30 & 50163.19 & 61723.29 \\
\hline 0 & -6 & 50127.11 & 61802.01 & 11 & -31 & 50163.19 & 61720.01 \\
\hline 0 & -7 & 50127.11 & 61798.73 & 11 & -32 & 50163.19 & 61716.73 \\
\hline 0 & -8 & 50127.11 & 61795.45 & 11 & -33 & 50163.19 & 61713.45 \\
\hline 0 & -9 & 50127.11 & 61792.17 & 11 & -34 & 50163.19 & 61710.17 \\
\hline 0 & -10 & 50127.11 & 61788.89 & 11 & -35 & 50163.19 & 61706.89 \\
\hline 0 & -11 & 50127.11 & 61785.61 & 11 & -36 & 50163.19 & 61703.61 \\
\hline 0 & -12 & 50127.11 & 61782.33 & 11 & -37 & 50.163 .19 & 61700.33 \\
\hline 0 & -13 & 50127.11 & 61779.05 & 11 & -38 & 50163.19 & 61697.05 \\
\hline 0 & -14 & 50127.11 & 61775.77 & 11 & -39 & 50163.19 & 61693.77 \\
\hline 0 & -15 & 50127.11 & 61772.49 & 11 & -40 & 50163.19 & 61690.49 \\
\hline 0 & -16 & 50127.11 & 61769.21 & 11 & -41 & 50163.19 & 61687.21 \\
\hline 0 & -17 & 50127.11 & 61765.93 & 11 & -42 & 50163.19 & 61683.93 \\
\hline 0 & -18 & 50127.11 & 61762.65 & 11 & -43 & 50163.19 & 61680.65 \\
\hline 0 & -19 & 50127.11 & 61759.37 & 11 & -44 & 50163.19 & 61677.37 \\
\hline 0 & -20 & 50127.11 & 61756.09 & 11 & -45 & 50163.19 & 61674.09 \\
\hline 0 & -21 & 50127.11 & 61752.81 & 12 & 0 & 50166.47 & 61821.69 \\
\hline 0 & -22 & 50127.11 & 61749.53 & 12 & -1 & 50166.47 & 61818.41 \\
\hline 0 & -23 & 50127.11 & 61746.25 & 12 & -2 & 50166.47 & 61815.13 \\
\hline 0 & -24 & 50127.11 & 61742.97 & 12 & -3 & 50166.47 & 61811.85 \\
\hline 0 & -25 & 50127.11 & 61739.69 & 12 & -4 & 50166.47 & 61808.57 \\
\hline 0 & -26 & 50127.11 & 61736.41 & 12 & -5 & 50166.47 & 61805.29 \\
\hline 0 & -27 & 50127.11 & 61733.13 & 12 & -6 & 50166.47 & 61802.01 \\
\hline 0 & -28 & 50127.11 & 61729.85 & 12 & -7 & 50166.47 & 61798.73 \\
\hline 0 & -29 & 50127.11 & 61726.57 & 12 & -8 & 50166.47 & 61795.45 \\
\hline 0 & -30 & 50127.11 & 61723.29 & 12 & -9 & 50166.47 & 61792.17 \\
\hline 0 & -31 & 50127.11 & 61720.01 & 12 & -10 & 50166.47 & 61788.89 \\
\hline 0 & -32 & 50127.11 & 61716.73 & 12 & -11 & 50166.47 & 61785.61 \\
\hline 0 & -33 & 50127.11 & 61713.45 & 12 & -12 & 50166.47 & 61782.33 \\
\hline 0 & -34 & 50127.11 & 61710.17 & 12 & -13 & 50166.47 & 61779.05 \\
\hline 0 & -35 & 50127.11 & 61706.89 & 12 & -14 & 50166.47 & 61775.77 \\
\hline 0 & -36 & 50127.11 & 61703.61 & 12 & -15 & 50166.47 & 61772.49 \\
\hline 0 & -37 & 50127.11 & 61700.33 & 12 & -16 & $50166 ̣ .47$ & 61769.21 \\
\hline 0 & -38 & 50127.11 & 61697.05 & 12 & -17 & $501+66.47$ & 61765.93 \\
\hline 0 & -39 & 50127.11 & 61693.77 & 12 & -18 & 50166.47 & 61762.65 \\
\hline 0 & -40 & 50127.11 & 61690.49 & 12 & -19 & 50166.47 & 61759.37 \\
\hline 0 & -41 & 50127.11 & 61687.21 & 12 & -20 & 50166.47 & 61756.09 \\
\hline 0 & -42 & 50127.11 & 61683.93 & 12 & -21 & 50166.47 & 61752.81 \\
\hline 0 & -43 & 50127.11 & 61680.65 & 12 & -22 & 50166.47 & 61749.53 \\
\hline 0 & -44 & 50127.11 & 61677.37 & 12 & -23 & 50166.47 & 61746.25 \\
\hline 0 & -45 & 50127.11 & 61674.09 & 12 & -24 & 50166.47 & 61742.97 \\
\hline 0 & -46 & 50127.11 & 61670.81 & 12 & -25 & 50166.47 & 61739.69 \\
\hline 0 & -47 & 50127.11 & 61667.53 & 12 & -26 & 50166.47 & 61736.41 \\
\hline
\end{tabular}


Hydroflouric Acid Spill Magnetic Survey Coordinate Transformatiom

\begin{tabular}{|c|c|c|c|c|c|c|c|}
\hline \multicolumn{2}{|c|}{ GRID } & \multicolumn{2}{|c|}{ SITE } & \multicolumn{2}{|c|}{ GRID } & \multicolumn{2}{|c|}{$\cdots \quad$ SITE } \\
\hline$x$ & $\mathbf{Y}$ & EASTING & NORTHING & $x$ & $\mathbf{Y}$ & EASTING & NORTHING \\
\hline 0 & -48 & 50127.11 & 61664.25 & 12 & -27 & 50166.47 & 61733.13 \\
\hline 0 & -49 & 50127.11 & 61660.97 & 12 & -28 & 50166.47 & 61729.85 \\
\hline 0 & -50 & 50127.11 & 61657.69 & 12 & -29 & 50166.47 & 61726.57 \\
\hline 0 & -51 & 50127.11 & 61654.41 & 12 & -30 & 50166.47 & 61723.29 \\
\hline 1 & 0 & 50130.39 & 61821.69 & 12 & -31 & 50166.47 & 61720.01 \\
\hline 1 & -1 & 50130.39 & 61818.41 & 12 & -32 & 50166.47 & 61716.73 \\
\hline 1 & -2 & 50130.39 & 61815.13 & 12 & -33 & 50166.47 & 61713.45 \\
\hline 1 & -3 & 50130.39 & 61811.85 & 12 & -34 & 50166.47 & 61710.17 \\
\hline 1 & -4 & 50130.39 & 61808.57 & 12 & -35 & 50166.47 & 61706.89 \\
\hline 1 & -5 & 50130.39 & 61805.29 & 12 & -36 & 50166.47 & 61703.61 \\
\hline 1 & -6 & 50130.39 & 61802.01 & 12 & -37 & 50166.47 & 61700.33 \\
\hline 1 & -7 & 50130.39 & 61798.73 & 12 & -38 & 50166.47 & 61697.05 \\
\hline 1 & -8 & 50130.39 & 61795.45 & 12 & -39 & 50166.47 & 61693.77 \\
\hline 1 & -9 & 50130.39 & 61792.17 & 12 & -40 & 50166.47 & 61690.49 \\
\hline 1 & -10 & 50130.39 & 61788.89 & 12 & -41 & 50166.47 & 61687.21 \\
\hline 1 & -11 & 50130.39 & 61785.61 & 12 & -42 & 50166.47 & 61683.93 \\
\hline 1 & -12 & 50130.39 & 61782.33 & 12 & -43 & 50166.47 & 61680.65 \\
\hline 1 & -13 & 50130.39 & 61779.05 & 13 & 0 & 50169.75 & 61821.69 \\
\hline 1 & -14 & 50130.39 & 61775.77 & 13 & -1 & 50169.75 & 61818.41 \\
\hline 1 & -15 & 50130.39 & 61772.49 & 13 & -2 & 50169.75 & 61815.13 \\
\hline 1 & -16 & 50130.39 & 61769.21 & 13 & -3 & 50169.75 & 61811.85 \\
\hline 1 & -17 & 50130.39 & 61765.93 & 13 & -4 & 50169.75 & 61808.57 \\
\hline 1 & -18 & 50130.39 & 61762.65 & 13 & -5 & 50169.75 & 61805.29 \\
\hline 1 & -19 & 50130.39 & 61759.37 & 13 & -6 & 50169.75 & 61802.01 \\
\hline 1 & -20 & 50130.39 & 61756.09 & 13 & -7 & 50169.75 & 61798.73 \\
\hline 1 & -21 & 50130.39 & 61752.81 & 13 & -8 & 50169.75 & 61795.45 \\
\hline 1 & -22 & 50130.39 & 61749.53 & 13 & -9 & 50169.75 & 61792.17 \\
\hline 1 & -23 & 50130.39 & 61746.25 & 13 & -10 & 50169.75 & 61788.89 \\
\hline 1 & -24 & 50130.39 & 61742.97 & 13 & -11 & 50169.75 & 61785.61 \\
\hline 1 & -25 & 50130.39 & 61739.69 & 13 & -12 & 50169.75 & 61782.33 \\
\hline 1 & -26 & 50130.39 & 61736.41 & 13 & -13 & 50169.75 & 61779.05 \\
\hline 1 & -27 & 50130.39 & 61733.13 & 13 & -14 & 50169.715 & 61775.77 \\
\hline 1 & -28 & 50130.39 & 61729.85 & 13 & -15 & 50169.75 & 61772.49 \\
\hline 1 & -29 & 50130.39 & 61726.57 & 13 & -16 & 50169.75 & 61769.21 \\
\hline 1 & -30 & 50130.39 & 61723.29 & 13 & -17 & 50169.75 & 61765.93 \\
\hline 1 & -31 & 50130.39 & 61720.01 & 13 & -18 & $50169.7 \dot{5}$ & 61762.65 \\
\hline 1 & -32 & 50130.39 & 61716.73 & 13 & -19 & 50169.75 & 61759.37 \\
\hline 1 & -33 & 50130.39 & 61713.45 & 13 & -20 & 50169.75 & 61756.09 \\
\hline 1 & -34 & 50130.39 & 61710.17 & 13 & -21 & $50169.7,5$ & 61752.81 \\
\hline 1 & -35 & 50130.39 & 61706.89 & 13 & -22 & 50169.75 & 61749.53 \\
\hline 1 & -36 & 50130.39 & 61703.61 & 13 & -23 & 50169.75 & 61746.25 \\
\hline 1 & -37 & 50130.39 & 61700.33 & 13 & -24 & 50169.75 & 61742.97 \\
\hline 1 & -38 & 50130.39 & 61697.05 & 13 & -25 & 50169.75 & 61739.69 \\
\hline 1 & -39 & 50130.39 & 61693.77 & 13 & -26 & 50169.75 & 61736.41 \\
\hline 1 & -40 & 50130.39 & 61690.49 & 13 & -27 & 50169.75 & 61733.13 \\
\hline 1 & -41 & 50130.39 & 61687.21 & 13 & -28 & 50169.75 & 61729.85 \\
\hline 1 & -42 & 50130.39 & 61683.93 & 13 & -29 & 50169.75 & 61726.57 \\
\hline 1 & -43 & 50130.39 & 61680.65 & 13 & -30 & 50169.75 & 61723.29 \\
\hline 1 & -44 & 50130.39 & 61677.37 & 13 & -31 & 50169.75 & 61720.01 \\
\hline & & & & & & $:$ & \\
\hline & & & & & & , & \\
\hline
\end{tabular}


Hydroflouric Acid Spill Magnetic Survey Coordinate Transformatiom

\begin{tabular}{|c|c|c|c|c|c|c|c|}
\hline \multicolumn{2}{|c|}{ GRID } & \multicolumn{2}{|c|}{ SITE } & \multicolumn{2}{|c|}{ GRID } & \multicolumn{2}{|c|}{ SITE } \\
\hline $\mathbf{x}$ & $Y$ & EASTING & NORTHING & $x$ & $\mathbf{Y}$ & EASTING & NORTHING \\
\hline 1 & -45 & 50130.39 & 61674.09 & 13 & -32 & 50169.75 & 61716.73 \\
\hline 1 & -46 & 50130.39 & 61670.81 & 13 & -33 & 50169.75 & 61713.45 \\
\hline 1 & -47 & 50130.39 & 61667.53 & 13 & -34 & 50169.75 & 61710.17 \\
\hline 1 & -48 & 50130.39 & 61664.25 & 13 & -35 & 50169.75 & 61706.89 \\
\hline 1 & -49 & 50130.39 & 61660.97 & 13 & -36 & 50169.75 & 61703.61 \\
\hline 1 & -50 & 50130.39 & 61657.69 & 13 & -37 & 50169.75 & 61700.33 \\
\hline 1 & -51 & 50130.39 & 61654.41 & 13 & -38 & 50169.75 & 61697.05 \\
\hline 1 & -52 & 50130.39 & 61651.13 & 13 & -39 & 50169.75 & 61693.77 \\
\hline 2 & 0 & 50133.67 & 61821.69 & 13 & -40 & 50169.75 & 61690.49 \\
\hline 2 & -1 & 50133.67 & 61818.41 & 13 & -41 & 50169.75 & 61687.21 \\
\hline 2 & -2 & 50133.67 & 61815.13 & 14 & 0 & 50173.03 & 61821.69 \\
\hline 2 & -3 & 50133.67 & 61811.85 & 14 & -1 & 50173.03 & 61818.41 \\
\hline 2 & -4 & 50133.67 & 61808.57 & 14 & -2 & 50173.03 & 61815.13 \\
\hline 2 & -5 & 50133.67 & 61805.29 & 14 & -3 & 50173.03 & 61811.85 \\
\hline 2 & -6 & 50133.67 & 61802.01 & 14 & -4 & 50173.03 & 61808.57 \\
\hline 2 & -7 & 50133.67 & 61798.73 & 14 & -5 & 50173.03 & 61805.29 \\
\hline 2 & -8 & 50133.67 & 61795.45 & 14 & -6 & 50173.03 & 61802.01 \\
\hline 2 & -9 & 50133.67 & 61792.17 & 14 & -7 & 50173.03 & 61798.73 \\
\hline 2 & -10 & 50133.67 & 61788.89 & 14 & -8 & 50173.03 & 61795.45 \\
\hline 2 & -11 & 50133.67 & 61785.61 & 14 & -9 & 50173.03 & 61792.17 \\
\hline 2 & -12 & 50133.67 & 61782.33 & 14 & -10 & 50173.03 & 61788.89 \\
\hline 2 & -13 & 50133.67 & 61779.05 & 14 & -11 & 50173.03 & 61785.61 \\
\hline 2 & -14 & 50133.67 & 61775.77 & 14 & -12 & 50173.03 & 61782.33 \\
\hline 2 & -15 & 50133.67 & 61772.49 & 14 & -13 & 50173.03 & 61779.05 \\
\hline 2 & -16 & 50133.67 & 61769.21 & 14 & -14 & 50173.03 & 61775.77 \\
\hline 2 & -17 & 50133.67 & 61765.93 & 14 & -15 & 50173.03 & 61772.49 \\
\hline 2 & -18 & 50133.67 & 61762.65 & 14 & -16 & 50173.03 & 61769.21 \\
\hline 2 & -19 & 50133.67 & 61759.37 & 14 & -17 & 50173.03 & 61765.93 \\
\hline 2 & -20 & 50133.67 & 61756.09 & 14 & -18 & 50173.03 & 61762.65 \\
\hline 2 & -21 & 50133.67 & 61752.81 & 14 & -19 & 50173.03 & 61759.37 \\
\hline 2 & -22 & 50133.67 & 61749.53 & 14 & -20 & 50173.03 & 61756.09 \\
\hline 2 & -23 & 50133.67 & 61746.25 & 14 & -21 & 50173.03 & 61752.81 \\
\hline 2 & -24 & 50133.67 & 61742.97 & 14 & -22 & 50173.03 & 61749.53 \\
\hline 2 & -25 & 50133.67 & 61739.69 & 14 & -23 & 50173.03 & 61746.25 \\
\hline 2 & -26 & 50133.67 & 61736.41 & 14 & -24 & 50173.03 & 61742.97 \\
\hline 2 & -27 & 50133.67 & 61733.13 & 14 & -25 & 50173.03 & 61739.69 \\
\hline 2 & -28 & 50133.67 & 61729.85 & 14 & -26 & 50173.03 & 61736.41 \\
\hline 2 & -29 & 50133.67 & 61726.57 & 14 & -27 & 50173.03 & 61733.13 \\
\hline 2 & -30 & 50133.67 & 61723.29 & 14 & -28 & 50173.03 & 61729.85 \\
\hline 2 & -31 & 50133.67 & 61720.01 & 14 & -29 & 50173.03 & 61726.57 \\
\hline 2 & -32 & 50133.67 & 61716.73 & 14 & -30 & 50173.03 & 61723.29 \\
\hline 2 & -33 & 50133.67 & 61713.45 & 14 & -31 & 50173.03 & 61720.01 \\
\hline 2 & -34 & 50133.67 & 61710.17 & 14 & -32 & 50173.03 & 61716.73 \\
\hline 2 & -35 & 50133.67 & 61706.89 & 14 & -33 & 50173.03 & 61713.45 \\
\hline 2 & -36 & 50133.67 & 61703.61 & 14 & -34 & 50173.03 & 61710.17 \\
\hline 2 & -37 & 50133.67 & 61700.33 & 14 & -35 & 50173.03 & 61706.89 \\
\hline 2 & -38 & 50133.67 & 61697.05 & 14 & -36 & 50173.03 & 61703.61 \\
\hline 2 & -39 & 50133.67 & 61693.77 & 14 & -37 & 50173.03 & 61700.33 \\
\hline 2 & -40 & 50133.67 & 61690.49 & 14 & -38 & 50173.03 & 61697.05 \\
\hline
\end{tabular}


Hydroflouric Acid Spill Magnetic Survey Coordinate Transformatiom

\begin{tabular}{|c|c|c|c|c|c|c|c|}
\hline \multicolumn{2}{|c|}{ GRID } & \multicolumn{2}{|c|}{ SITE } & \multicolumn{2}{|c|}{ GRID } & \multicolumn{2}{|c|}{ SITE } \\
\hline $\mathbf{x}$ & $\mathbf{Y}$ & EASTING & NORTHING & $x$ & $\mathbf{Y}$ & EASTING & NORTHINC \\
\hline 2 & -41 & 50133.67 & 61687.21 & 14 & -39 & 50173.03 & 61693.77 \\
\hline 2 & -42 & 50133.67 & 61683.93 & 14 & -40 & 50173.03 & 61690.49 \\
\hline 2 & -43 & 50133.67 & 61680.65 & 15 & 0 & 50176.31 & 61821.69 \\
\hline 2 & -44 & 50133.67 & 61677.37 & 15 & -1 & 50176.31 & 61818.41 \\
\hline 2 & -45 & 50133.67 & 61674.09 & 15 & -2 & 50176.31 & 61815.13 \\
\hline 2 & -46 & 50133.67 & 61670.81 & 15 & -3 & 50176.31 & 61811.85 \\
\hline 2 & -47 & 50133.67 & 61667.53 & 15 & -4 & 50176.31 & 61808.57 \\
\hline 2 & -48 & 50133.67 & 61664.25 & 15 & -5 & 50176.31 & 61805.29 \\
\hline 2 & -49 & 50133.67 & 61660.97 & 15 & -6 & 50176.31 & 61802.01 \\
\hline 2 & -50 & 50133.67 & 61657.69 & 15 & -7 & 50176.31 & 61798.73 \\
\hline 2 & -51 & 50133.67 & 61654.41 & 15 & -8 & 50176.31 & 61795.45 \\
\hline 2 & -52 & 50133.67 & 61651.13 & 15 & -9 & 50176.31 & 61792.17 \\
\hline 3 & 0 & 50136.95 & 61821.69 & 15 & -10 & 50176.31 & 61788.89 \\
\hline 3 & -1 & 50136.95 & 61818.41 & 15 & -11 & 50176.31 & 61785.61 \\
\hline 3 & -2 & 50136.95 & 61815.13 & 15 & -12 & 50176.31 & 61782.33 \\
\hline 3 & -3 & 50136.95 & 61811.85 & 15 & -13 & 50176.31 & 61779.05 \\
\hline 3 & -4 & 50136.95 & 61808.57 & 15 & -14 & 50176.31 & 61775.77 \\
\hline 3 & -5 & 50136.95 & 61805.29 & 15 & -15 & 50176.31 & 61772.49 \\
\hline 3 & -6 & 50136.95 & 61802.01 & 15 & -16 & 50176.31 & 61769.21 \\
\hline 3 & -7 & 50136.95 & 61798.73 & 15 & -17 & 50176.31 & 61765.93 \\
\hline 3 & -8 & 50136.95 & 61795.45 & 15 & -18 & 50176.317 & 61762.65 \\
\hline 3 & -9 & 50136.95 & 61792.17 & 15 & -19 & 50176.31 & 61759.37 \\
\hline 3 & -10 & 50136.95 & 61788.89 & 15 & -20 & 50176.31 & 61756.09 \\
\hline 3 & -11 & 50136.95 & 61785.61 & 15 & -21 & 50176.31 & 61752.81 \\
\hline 3 & -12 & 50136.95 & 61782.33 & 15 & -22 & 50176.31 & 61749.53 \\
\hline 3 & -13 & 50136.95 & 61779.05 & 15 & -23 & 50176.31 & 61746.25 \\
\hline 3 & -14 & 50136.95 & 61775.77 & 15 & -24 & 50176.31 & 61742.97 \\
\hline 3 & -15 & 50136.95 & 61772.49 & 15 & -25 & 50176.31 & 61739.69 \\
\hline 3 & -16 & 50136.95 & 61769.21 & 15 & -26 & 50176.31 & 61736.41 \\
\hline 3 & -17 & 50136.95 & 61765.93 & 15 & -27 & 50176.31 & 61733.13 \\
\hline 3 & -18 & 50136.95 & 61762.65 & 15 & -28 & 50176.31 & 61729.85 \\
\hline 3 & -19 & 50136.95 & 61759.37 & 15 & -29 & 50176.31 & 61726.57 \\
\hline 3 & -20 & 50136.95 & 61756.09 & 15 & -30 & 50176.31 & 61723.29 \\
\hline 3 & -21 & 50136.95 & 61752.81 & 15 & -31 & 50176.31 & 61720.01 \\
\hline 3 & -22 & 50136.95 & 61749.53 & 15 & -32 & 50176.31 & 61716.73 \\
\hline 3 & -23 & 50136.95 & 61746.25 & 15 & -33 & 50176.31 & 61713.45 \\
\hline 3 & -24 & 50136.95 & 61742.97 & 15 & -34 & 50176.31 & 61710.17 \\
\hline 3 & -25 & 50136.95 & 61739.69 & 15 & -35 & 50176.31 & 61706.89 \\
\hline 3 & -26 & 50136.95 & 61736.41 & 15 & -36 & 50176.31 & 61703.61 \\
\hline 3 & -27 & 50136.95 & 61733.13 & 15 & -37 & 50176.31 & 61700.33 \\
\hline 3 & -28 & 50136.95 & 61729.85 & 15 & -38 & 50176.31 & 61697.05 \\
\hline 3 & -29 & 50136.95 & 61726.57 & 16 & 0 & 50179.59 & 61821.69 \\
\hline 3 & -30 & 50136.95 & 61723.29 & 16 & -1 & 50179.59 & 61818.41 \\
\hline 3 & -31 & 50136.95 & 61720.01 & 16 & -2 & 50179.59 & 61815.13 \\
\hline 3 & -32 & 50136.95 & 61716.73 & 16 & -3 & 50179.59 & 61811.85 \\
\hline 3 & -33 & 50136.95 & 61713.45 & 16 & -4 & 50179.59 & 61808.57 \\
\hline 3 & -34 & 50136.95 & 61710.17 & 16 & -5 & 50179.59 & 61805.29 \\
\hline 3 & -35 & 50136.95 & 61706.89 & 16 & -6 & 50179.59 & 61802.01 \\
\hline 3 & -36 & 50136.95 & 61703.61 & 16 & -7 & 50179.59 & 61798.73 \\
\hline
\end{tabular}


Hydroflouric Acid Spill Magnetic Survey Coordinate Transformatiom

\begin{tabular}{|c|c|c|c|c|c|c|c|}
\hline \multicolumn{2}{|c|}{ GRID } & \multicolumn{2}{|c|}{ SITE } & \multicolumn{2}{|c|}{ GRID } & \multicolumn{2}{|c|}{ SITE } \\
\hline$x$ & $Y$ & EASTING & NORTHING & $x$ & $Y$ & EASTING & NORTHING \\
\hline 3 & -37 & 50136.95 & 61700.33 & 16 & -8 & 50179.59 & 61795.45 \\
\hline 3 & -38 & 50136.95 & 61697.05 & 16 & -9 & 50179.59 & 61792.17 \\
\hline 3 & -39 & 50136.95 & 61693.77 & 16 & -10 & 50179.59 & 61788.89 \\
\hline 3 & -40 & 50136.95 & 61690.49 & 16 & -11 & 50179.59 & 61785.61 \\
\hline 3 & -41 & 50136.95 & 61687.21 & 16 & -12 & 50179.59 & 61782.33 \\
\hline 3 & -42 & 50136.95 & 61683.93 & 16 & -13 & 50179.59 & 61779.05 \\
\hline 3 & -43 & 50136.95 & 61680.65 & 16 & -14 & 50179.59 & 61775.77 \\
\hline 3 & -44 & 50136.95 & 61677.37 & 16 & -15 & 50179.59 & 61772.49 \\
\hline 3 & -45 & 50136.95 & 61674.09 & 16 & -16 & 50179.59 & 61769.21 \\
\hline 3 & -46 & 50136.95 & 61670.81 & 16 & -17 & 50179.59 & 61765.93 \\
\hline 3 & -47 & 50136.95 & 61667.53 & 16 & -18 & 50179.59 & 61762.65 \\
\hline 3 & -48 & 50136.95 & 61664.25 & 16 & -19 & 50179.59 & 61759.37 \\
\hline 3 & -49 & 50136.95 & 61660.97 & 16 & -20 & 50179.59 & 61756.09 \\
\hline 3 & -50 & 50136.95 & 61657.69 & 16 & -21 & 50179.59 & 61752.81 \\
\hline 3 & -51 & 50136.95 & 61654.41 & 16 & -22 & 50179.59 & 61749.53 \\
\hline 3 & -52 & 50136.95 & 61651.13 & 16 & -23 & 50179.59 & 61746.25 \\
\hline 4 & 0 & 50140.23 & 61821.69 & 16 & -24 & 50179.59 & 61742.97 \\
\hline 4 & -1 & 50140.23 & 61818.41 & 16 & -25 & 50179.59 & 61739.69 \\
\hline 4 & -2 & 50140.23 & 61815.13 & 16 & -26 & 50179.59 & 61736.41 \\
\hline 4 & -3 & 50140.23 & 61811.85 & 16 & -27 & 50179.59 & 61733.13 \\
\hline 4 & -4 & 50140.23 & 61808.57 & 16 & -28 & 50179.59 & 61729.85 \\
\hline 4 & -5 & 50140.23 & 61805.29 & 16 & -29 & 50179.59 & 61726.57 \\
\hline 4 & -6 & 50140.23 & 61802.01 & 16 & -30 & 50179.59 & 61723.29 \\
\hline 4 & -7 & 50140.23 & 61798.73 & 16 & -31 & 50179.59 & 61720.01 \\
\hline 4 & -8 & 50140.23 & 61795.45 & 16 & -32 & 50179.59 & 61716.73 \\
\hline 4 & -9 & 50140.23 & 61792.17 & 16 & -33 & 50179.59 & 61713.45 \\
\hline 4 & -10 & 50140.23 & 61788.89 & 16 & -34 & 50179.59 & 61710.17 \\
\hline 4 & -11 & 50140.23 & 61785.61 & 16 & -35 & 50179.59 & 61706.89 \\
\hline 4 & -12 & 50140.23 & 61782.33 & 16 & -36 & 50179.59 & 61703.61 \\
\hline 4 & -13 & 50140.23 & 61779.05 & 17 & 0 & 50182.87 & 61821.69 \\
\hline 4 & -14 & 50140.23 & 61775.77 & 17 & -1 & 50182.87 & 61818.41 \\
\hline 4 & -15 & 50140.23 & 61772.49 & 17 & -2 & 50182.87 & 61815.13 \\
\hline 4 & -16 & 50140.23 & 61769.21 & 17 & -3 & 50182.87 & 61811.85 \\
\hline 4 & -17 & 50140.23 & 61765.93 & 17 & -4 & 50182.87 & 61808.57 \\
\hline 4 & -18 & 50140.23 & 61762.65 & 17 & -5 & 50182.87 & 61805.29 \\
\hline 4 & -19 & 50140.23 & 61759.37 & 17 & -6 & 50182.87 & 61802.01 \\
\hline 4 & -20 & 50140.23 & 61756.09 & 17 & -7 & 50182.87 & 61798.73 \\
\hline 4 & -21 & 50140.23 & 61752.81 & 17 & -8 & 50182.87 & 61795.45 \\
\hline 4 & -22 & 50140.23 & 61749.53 & 17 & -9 & 50182.87 & 61792.17 \\
\hline 4 & -23 & 50140.23 & 61746.25 & 17 & -10 & 50182.87 & 61788.89 \\
\hline 4 & -24 & 50140.23 & 61742.97 & 17 & -11 & 50182.87 & 61785.61 \\
\hline 4 & -25 & 50140.23 & 61739.69 & 17 & -12 & 50182.87 & 61782.33 \\
\hline 4 & -26 & 50140.23 & 61736.41 & 17 & -13 & 50182.87 & 61779.05 \\
\hline 4 & -27 & 50140.23 & 61733.13 & 17 & -14 & 50182.87 & 61775.77 \\
\hline 4 & -28 & 50140.23 & 61729.85 & 17 & -15 & 50182.87 & 61772.49 \\
\hline 4 & -29 & 50140.23 & 61726.57 & 17 & -16 & 50182.87 & 61769.21 \\
\hline 4 & -30 & 50140.23 & 61723.29 & 17 & -17 & 50182.87 & 61765.93 \\
\hline 4 & -31 & 50140.23 & 61720.01 & 17 & -18 & 50182.87 & 61762.65 \\
\hline 4 & -32 & 50140.23 & 61716.73 & 17 & -19 & 50182.87 & 61759.37 \\
\hline & & & & & & $\begin{array}{l}: \quad \ldots \\
: \quad: . .\end{array}$ & \\
\hline & & & & & & : & \\
\hline
\end{tabular}


Hydroflouric Acid Spill Magnetic Survey Coordinate Transformatiom

\begin{tabular}{|c|c|c|c|c|c|c|c|}
\hline \multicolumn{2}{|c|}{ GRID } & \multicolumn{2}{|c|}{ SITE } & \multicolumn{2}{|c|}{ GRID } & \multicolumn{2}{|c|}{ SITE } \\
\hline$x$ & $Y$ & EASTING & NORTHING & $\mathbf{x}$ & $\mathbf{Y}$ & EASTING & NORTHING \\
\hline 4 & -33 & 50140.23 & 61713.45 & 17 & -20 & 50182.87 & 61756.09 \\
\hline 4 & -34 & 50140.23 & 61710.17 & 17 & -21 & 50182.87 & 61752.81 \\
\hline 4 & -35 & 50140.23 & 61706.89 & 17 & -22 & 50182.87 & 61749.53 \\
\hline 4 & -36 & 50140.23 & 61703.61 & 17 & -23 & 50182.87 & 61746.25 \\
\hline 4 & -37 & 50140.23 & 61700.33 & 17 & -24 & 50182.87 & 61742.97 \\
\hline 4 & -38 & 50140.23 & 61697.05 & 17 & -25 & 50182.87 & 61739.69 \\
\hline 4 & -39 & 50140.23 & 61693.77 & 17 & -26 & 50182.87 & 61736.41 \\
\hline 4 & -40 & 50140.23 & 61690.49 & 17 & -27 & 50182.87 & 61733.13 \\
\hline 4 & -41 & 50140.23 & 61687.21 & 17 & -28 & 50182.87 & 61729.85 \\
\hline 4 & -42 & 50140.23 & 61683.93 & 17 & -29 & 50182.87 & 61726.57 \\
\hline 4 & -43 & 50140.23 & 61680.65 & 17 & -30 & 50182.87 & 61723.29 \\
\hline 4 & -44 & 50140.23 & 61677.37 & 17 & -31 & 50182.87 & 61720.01 \\
\hline 4 & -45 & 50140.23 & 61674.09 & 17 & -32 & 50182.87 & 61716.73 \\
\hline 4 & -46 & 50140.23 & 61670.81 & 17 & -33 & 50182.87 & 61713.45 \\
\hline 4 & -47 & 50140.23 & 61667.53 & 17 & -34 & 50182.87 & 61710.17 \\
\hline 4 & -48 & 50140.23 & 61664.25 & 18 & 0 & 50186.15 & 61821.69 \\
\hline 4 & -49 & 50140.23 & 61660.97 & 18 & -1 & 50186.15 & 61818.41 \\
\hline 4 & -50 & 50140.23 & 61657.69 & 18 & -2 & 50186.15 & 61815.13 \\
\hline 4 & -51 & 50140.23 & 61654.41 & 18 & -3 & 50186.15 & 61811.85 \\
\hline 4 & -52 & 50140.23 & 61651.13 & 18 & -4 & $50186.1,5$ & 61808.57 \\
\hline 5 & 0 & 50143.51 & 61821.69 & 18 & -5 & 50186.15 & 61805.29 \\
\hline 5 & -1 & 50143.51 & 61818.41 & 18 & -6 & 50186.15 & 61802.01 \\
\hline 5 & -2 & 50143.51 & 61815.13 & 18 & -7 & 50186.15 & 61798.73 \\
\hline 5 & -3 & 50143.51 & 61811.85 & 18 & -8 & 50186.15 & 61795.45 \\
\hline 5 & -4 & 50143.51 & 61808.57 & 18 & -9 & 50186.15 & 61792.17 \\
\hline 5 & -5 & 50143.51 & 61805.29 & 18 & -10 & 50186.15 & 61788.89 \\
\hline 5 & -6 & 50143.51 & 61802.01 & 18 & -11 & 50186.15 & 61785.61 \\
\hline 5 & -7 & 50143.51 & 61798.73 & 18 & -12 & 50186.15 & 61782.33 \\
\hline 5 & -8 & 50143.51 & 61795.45 & 18 & -13 & 50186.15 & 61779.05 \\
\hline 5 & -9 & 50143.51 & 61792.17 & 18 & -14 & 50186.15 & 61775.77 \\
\hline 5 & -10 & 50143.51 & 61788.89 & 18 & -15 & 50186.15 & 61772.49 \\
\hline 5 & -11 & 50143.51 & 61785.61 & 18 & -16 & 50186.15 & 61769.21 \\
\hline 5 & -12 & 50143.51 & 61782.33 & 18 & -17 & 50186.15 & 61765.93 \\
\hline 5 & -13 & 50143.51 & 61779.05 & 18 & -18 & 50186.15 & 61762.65 \\
\hline 5 & -14 & 50143.51 & 61775.77 & 18 & -19 & 50186.15 & 61759.37 \\
\hline 5 & -15 & 50143.51 & 61772.49 & 18 & -20 & 50186.15 & 61756.09 \\
\hline 5 & -16 & 50143.51 & 61769.21 & 18 & -21 & 50186.15 & 61752.81 \\
\hline 5 & -17 & 50143.51 & 61765.93 & 18 & -22 & 50186.15 & 61749.53 \\
\hline 5 & -18 & 50143.51 & 61762.65 & 18 & -23 & 50186.15 & 61746.25 \\
\hline 5 & -19 & 50143.51 & 61759.37 & 18 & -24 & 50186.15 & 61742.97 \\
\hline 5 & -20 & 50143.51 & 61756.09 & 18 & -25 & 50186.15 & 61739.69 \\
\hline 5 & -21 & 50143.51 & 61752.81 & 18 & -26 & 50186.15 & 61736.41 \\
\hline 5 & -22 & 50143.51 & 61749.53 & 18 & -27 & 50186.15 & 61733.13 \\
\hline 5 & -23 & 50143.51 & 61746.25 & 18 & -28 & 50186.15 & 61729.85 \\
\hline 5 & -24 & 50143.51 & 61742.97 & 18 & -29 & 50186.15 & 61726.57 \\
\hline 5 & -25 & 50143.51 & 61739.69 & 18 & -30 & 50186.15 & 61723.29 \\
\hline 5 & -26 & 50143.51 & 61736.41 & 18 & -31 & 50186.15 & 61720.01 \\
\hline 5 & -27 & 50143.51 & 61733.13 & 18 & -32 & 50186.15 & 61716.73 \\
\hline 5 & -28 & 50143.51 & 61729.85 & 18 & -33 & 50186.15 & 61713.45 \\
\hline
\end{tabular}


Hydroflouric Acid Spill Magnetic Survey Coordinate Transformatiom

\begin{tabular}{|c|c|c|c|c|c|c|c|}
\hline \multicolumn{2}{|c|}{ GRID } & \multicolumn{2}{|c|}{ SITE } & \multicolumn{2}{|c|}{ GRID } & \multicolumn{2}{|c|}{ SITE } \\
\hline$x$ & $\mathbf{Y}$ & EASTING & NORTHING & $x$ & $\mathbf{Y}$ & EASTING & NORTHINC \\
\hline 5 & -29 & 50143.51 & 61726.57 & 18 & -34 & 50186.15 & 61710.17 \\
\hline 5 & -30 & 50143.51 & 61723.29 & 19 & 0 & 50189.43 & 61821.69 \\
\hline 5 & -31 & 50143.51 & 61720.01 & 19 & -1 & 50189.43 & 61818.41 \\
\hline 5 & -32 & 50143.51 & 61716.73 & 19 & -2 & 50189.43 & 61815.13 \\
\hline 5 & -33 & 50143.51 & 61713.45 & 19 & -3 & 50189.43 & 61811.85 \\
\hline 5 & -34 & 50143.51 & 61710.17 & 19 & -4 & 50189.43 & 61808.57 \\
\hline 5 & -35 & 50143.51 & 61706.89 & 19 & -5 & 50189.43 & 61805.29 \\
\hline 5 & -36 & 50143.51 & 61703.61 & 19 & -6 & 50189.43 & 61802.01 \\
\hline 5 & -37 & 50143.51 & 61700.33 & 19 & -7 & 50189.43 & 61798.73 \\
\hline 5 & -38 & 50143.51 & 61697.05 & 19 & -8 & 50189.43 & 61795.45 \\
\hline 5 & -39 & 50143.51 & 61693.77 & 19 & -9 & 50189.43 & 61792.17 \\
\hline 5 & -40 & 50143.51 & 61690.49 & 19 & -10 & 50189.43 & 61788.89 \\
\hline 5 & -41 & 50143.51 & 61687.21 & 19 & -11 & 50189.43 & 61785.61 \\
\hline 5 & -42 & 50143.51 & 61683.93 & 19 & -12 & 50189.43 & 61782.33 \\
\hline 5 & -43 & 50143.51 & 61680.65 & 19 & -13 & 50189.43 & 61779.05 \\
\hline 5 & -44 & 50143.51 & 61677.37 & 19 & -14 & 50189.43 & 61775.77 \\
\hline 5 & -45 & 50143.51 & 61674.09 & 19 & -15 & 50189.43 & 61772.49 \\
\hline 5 & -46 & 50143.51 & 61670.81 & 19 & -16 & 50189.43 & 61769.21 \\
\hline 5 & -47 & 50143.51 & 61667.53 & 19 & -17 & 50189.43 & 61765.93 \\
\hline 5 & -48 & 50143.51 & 61664.25 & 19 & -18 & 50189.43 & 61762.65 \\
\hline 5 & -49 & 50143.51 & 61660.97 & 19 & -19 & 50189.43 & 61759.37 \\
\hline 5 & -50 & 50143.51 & 61657.69 & 19 & -20 & 50189.43 & 61756.09 \\
\hline 5 & -51 & 50143.51 & 61654.41 & 19 & -21 & 50189.43 & 61752.81 \\
\hline 6 & 0 & 50146.79 & 61821.69 & 19 & -22 & 50189.43 & 61749.53 \\
\hline 6 & -1 & 50146.79 & 61818.41 & 19 & -23 & 50189.43 & 61746.25 \\
\hline 6 & -2 & 50146.79 & 61815.13 & 19 & -24 & 50189.43 & 61742.97 \\
\hline 6 & -3 & 50146.79 & 61811.85 & 19 & -25 & 50189.43 & 61739.69 \\
\hline 6 & -4 & 50146.79 & 61808.57 & 19 & -26 & 50189.43 & 61736.41 \\
\hline 6 & -5 & 50146.79 & 61805.29 & 19 & -27 & 50189.43 & 61733.13 \\
\hline 6 & -6 & 50146.79 & 61802.01 & 19 & -28 & 50189.43 & 61729.85 \\
\hline 6 & -7 & 50146.79 & 61798.73 & 19 & -29 & 50189.43 & 61726.57 \\
\hline 6 & -8 & 50146.79 & 61795.45 & 19 & -30 & 50189.43 & 61723.29 \\
\hline 6 & -9 & 50146.79 & 61792.17 & 19 & -31 & 50189.43 & 61720.01 \\
\hline 6 & -10 & 50146.79 & 61788.89 & 19 & -32 & 50189.43 & 61716.73 \\
\hline 6 & -11 & 50146.79 & 61785.61 & 19 & -33 & 50189.43 & 61713.45 \\
\hline 6 & -12 & 50146.79 & 61782.33 & 20 & 0 & 50192.71 & 61821.69 \\
\hline 6 & -13 & 50146.79 & 61779.05 & 20 & -1 & 50192.71 & 61818.41 \\
\hline 6 & -14 & 50146.79 & 61775.77 & 20 & -2 & 50192.71 & 61815.13 \\
\hline 6 & -15 & 50146.79 & 61772.49 & 20 & -3 & 50192.71 & 61811.85 \\
\hline 6 & -16 & 50146.79 & 61769.21 & 20 & -4 & 50192.71 & 61808.57 \\
\hline 6 & -17 & 50146.79 & 61765.93 & 20 & -5 & 50192.71 & 61805.29 \\
\hline 6 & -18 & 50146.79 & 61762.65 & 20 & -6 & 50192.71 & 61802.01 \\
\hline 6 & -19 & 50146.79 & 61759.37 & 20 & -7 & 50192.71 & 61798.73 \\
\hline 6 & -20 & 50146.79 & 61756.09 & 20 & -8 & 50192.71 & 61795.45 \\
\hline 6 & -21 & 50146.79 & 61752.81 & 20 & -9 & 50192.71 & 61792.17 \\
\hline 6 & -22 & 50146.79 & 61749.53 & 20 & -10 & 50192.71 & 61788.89 \\
\hline 6 & -23 & 50146.79 & 61746.25 & 20 & -11 & 50192.71 & 61785.61 \\
\hline 6 & -24 & 50146.79 & 61742.97 & 20 & -12 & 50192.71 & 61782.33 \\
\hline 6 & -25 & 50146.79 & 61739.69 & 20 & -13 & 50192.71 & 61779.05 \\
\hline
\end{tabular}


Hydroflouric Acid Spill Magnetic Survey Coordinate Transformatiom

\begin{tabular}{|c|c|c|c|c|c|c|c|}
\hline \multicolumn{2}{|c|}{ GRID } & \multicolumn{2}{|c|}{ SITE } & \multicolumn{2}{|c|}{ GRID } & \multicolumn{2}{|c|}{ SITE } \\
\hline $\mathbf{x}$ & $\mathbf{Y}$ & EASTING & NORTHING & $\mathbf{x}$ & $\mathbf{Y}$ & EASTING I & NORTHING \\
\hline 6 & -26 & 50146.79 & 61736.41 & 20 & -14 & 50192.71 & 61775.77 \\
\hline 6 & -27 & 50146.79 & 61733.13 & 20 & -15 & 50192.71 & 61772.49 \\
\hline 6 & -28 & 50146.79 & 61729.85 & 20 & -16 & 50192.71 & 61769.21 \\
\hline 6 & -29 & 50146.79 & 61726.57 & 20 & -17 & 50192.71 & 61765.93 \\
\hline 6 & -30 & 50146.79 & 61723.29 & 20 & -18 & 50192.71 & 61762.65 \\
\hline 6 & -31 & 50146.79 & 61720.01 & 20 & -19 & 50192.71 & 61759.37 \\
\hline 6 & -32 & 50146.79 & 61716.73 & 20 & -20 & 50192.71 & 61756.09 \\
\hline 6 & -33 & 50146.79 & 61713.45 & 20 & -21 & 50192.71 & 61752.81 \\
\hline 6 & -34 & 50146.79 & 61710.17 & 20 & -22 & 50192.71 & 61749.53 \\
\hline 6 & -35 & 50146.79 & 61706.89 & 20 & -23 & 50192.71 & 61746.25 \\
\hline 6 & -36 & 50146.79 & 61703.61 & 20 & -24 & 50192.71 & 61742.97 \\
\hline 6 & -37 & 50146.79 & 61700.33 & 20 & -25 & 50192.71 & 61739.69 \\
\hline 6 & -38 & 50146.79 & 61697.05 & 20 & -26 & 50192.71 & 61736.41 \\
\hline 6 & -39 & 50146.79 & 61693.77 & 20 & -27 & 50192.71 & 61733.13 \\
\hline 6 & -40 & 50146.79 & 61690.49 & 20 & -28 & 50192.71 & 61729.85 \\
\hline 6 & -41 & 50146.79 & 61687.21 & 20 & -29 & 50192.71 & 61726.57 \\
\hline 6 & -42 & 50146.79 & 61683.93 & 20 & -30 & 50192.71 & 61723.29 \\
\hline 6 & -43 & 50146.79 & 61680.65 & 20 & -31 & 50192.71 & 61720.01 \\
\hline 6 & -44 & 50146.79 & 61677.37 & 21 & 0 & 50195.99 & 61821.69 \\
\hline 6 & -45 & 50146.79 & 61674.09 & 21 & -1 & 50195.99 & 61818.41 \\
\hline 6 & -46 & 50146.79 & 61670.81 & 21 & -2 & 50195.99 & 61815.13 \\
\hline 6 & -47 & 50146.79 & 61667.53 & 21 & -3 & 50195.99 & 61811.85 \\
\hline 6 & -48 & 50146.79 & 61664.25 & 21 & -4 & 50195.99 & 61808.57 \\
\hline 7 & 0 & 50150.07 & 61821.69 & 21 & -5 & 50195.99 & 61805.29 \\
\hline 7 & -1 & 50150.07 & 61818.41 & 21 & -6 & 50195.99 & 61802.01 \\
\hline 7 & -2 & 50150.07 & 61815.13 & 21 & -7 & 50195.99 & 61798.73 \\
\hline 7 & -3 & 50150.07 & 61811.85 & 21 & -8 & 50195.99 & 61795.45 \\
\hline 7 & -4 & 50150.07 & 61808.57 & 21 & -9 & 50195.99 & 61792.17 \\
\hline 7 & -5 & 50150.07 & 61805.29 & 21 & -10 & 50195.99 & 61788.89 \\
\hline 7 & -6 & 50150.07 & 61802.01 & 21 & -11 & 50195.99 & 61785.61 \\
\hline 7 & -7 & 50150.07 & 61798.73 & 21 & -12 & 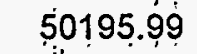 & 61782.33 \\
\hline 7 & -8 & 50150.07 & 61795.45 & 21 & -13 & 50195.99 & 61779.05 \\
\hline 7 & -9 & 50150.07 & 61792.17 & 21 & -14 & 50195.99 & 61775.77 \\
\hline 7 & -10 & 50150.07 & 61788.89 & 21 & -15 & 50195.99 & 61772.49 \\
\hline 7 & -11 & 50150.07 & 61785.61 & 21 & -16 & 50195.99 & 61769.21 \\
\hline 7 & -12 & 50150.07 & 61782.33 & 21 & -17 & 50195.99 & 61765.93 \\
\hline 7 & -13 & 50150.07 & 61779.05 & 21 & -18 & 50195.99 & 61762.65 \\
\hline 7 & -14 & 50150.07 & 61775.77 & 21 & -19 & 50195.99 & 61759.37 \\
\hline 7 & -15 & 50150.07 & 61772.49 & 21 & -20 & 50195.99 & 61756.09 \\
\hline 7 & -16 & 50150.07 & 61769.21 & 21 & -21 & 50195.99 & 61752.81 \\
\hline 7 & -17 & 50150.07 & 61765.93 & 21 & -22 & 50195.99 & 61749.53 \\
\hline 7 & -18 & 50150.07 & 61762.65 & 21 & -23 & 50195.99 & 61746.25 \\
\hline 7 & -19 & 50150.07 & 61759.37 & 21 & -24 & 50195.99 & 61742.97 \\
\hline 7 & -20 & 50150.07 & 61756.09 & 21 & -25 & 5़०195.99 & 61739.69 \\
\hline 7 & -21 & 50150.07 & 61752.81 & 21 & -26 & 50195.99 & 61736.41 \\
\hline 7 & -22 & 50150.07 & 61749.53 & 21 & -27 & 50195.99 & 61733.13 \\
\hline 7 & -23 & 50150.07 & 61746.25 & 21 & -28 & 50195.99 & 61729.85 \\
\hline 7 & -24 & 50150.07 & 61742.97 & 21 & -29 & 50195.99 & 61726.57 \\
\hline 7 & -25 & 50150.07 & 61739.69 & 21 & -30 & 50195.99 & 61723.29 \\
\hline
\end{tabular}


Hydroflouric Acid Spill Magnetic Survey Coordinate Transformatiom

\begin{tabular}{|c|c|c|c|c|c|c|c|}
\hline \multicolumn{2}{|c|}{ GRID } & \multicolumn{2}{|c|}{ SITE } & \multicolumn{2}{|c|}{ GRID } & \multicolumn{2}{|c|}{ SITE } \\
\hline$x$ & $\mathbf{Y}$ & EASTING & NORTHING & $\mathbf{x}$ & $\mathbf{Y}$ & EASTING & NORTHING \\
\hline 7 & -26 & 50150.07 & 61736.41 & 22 & 0 & 50199.27 & 61821.69 \\
\hline 7 & -27 & 50150.07 & 61733.13 & 22 & -1 & 50199.27 & 61818.41 \\
\hline 7 & -28 & 50150.07 & 61729.85 & 22 & -2 & 50199.27 & 61815.13 \\
\hline 7 & -29 & 50150.07 & 61726.57 & 22 & -3 & 50199.27 & 61811.85 \\
\hline 7 & -30 & 50150.07 & 61723.29 & 22 & -4 & 50199.27 & 61808.57 \\
\hline 7 & -31 & 50150.07 & 61720.01 & 22 & -5 & 50199.27 & 61805.29 \\
\hline 7 & -32 & 50150.07 & 61716.73 & 22 & -6 & 50199.27 & 61802.01 \\
\hline 7 & -33 & 50150.07 & 61713.45 & 22 & -7 & 50199.27 & 61798.73 \\
\hline 7 & -34 & 50150.07 & 61710.17 & 22 & -8 & 50199.27 & 61795.45 \\
\hline 7 & -35 & 50150.07 & 61706.89 & 22 & -9 & 50199.27 & 61792.17 \\
\hline 7 & -36 & 50150.07 & 61703.61 & 22 & -10 & 50199.27 & 61788.89 \\
\hline 7 & -37 & 50150.07 & 61700.33 & 22 & -11 & 50199.27 & 61785.61 \\
\hline 7 & -38 & 50150.07 & 61697.05 & 22 & -12 & 50199.27 & 61782.33 \\
\hline 7 & -39 & 50150.07 & 61693.77 & 22 & -13 & 50199.27 & 61779.05 \\
\hline 7 & -40 & 50150.07 & 61690.49 & 22 & -14 & 50199.27 & 61775.77 \\
\hline 7 & -41 & 50150.07 & 61687.21 & 22 & -15 & 50199.27 & 61772.49 \\
\hline 7 & -42 & 50150.07 & 61683.93 & 22 & -16 & 50199.27 & 61769.21 \\
\hline 7 & -43 & 50150.07 & 61680.65 & 22 & -17 & 50199.27 & 61765.93 \\
\hline 7 & -44 & 50150.07 & 61677.37 & 22 & -18 & 50199.27 & 61762.65 \\
\hline 7 & -45 & 50150.07 & 61674.09 & 22 & -19 & 50199.27 & 61759.37 \\
\hline 7 & -46 & 50150.07 & 61670.81 & 22 & -20 & 50199.27 & 61756.09 \\
\hline 7 & -47 & 50150.07 & 61667.53 & 22 & -21 & 50199.27 & 61752.81 \\
\hline 7 & -48 & 50150.07 & 61664.25 & 22 & -22 & 50199.27 & 61749.53 \\
\hline 7 & -49 & 50150.07 & 61660.97 & 22 & -23 & 50199.27 & 61746.25 \\
\hline 7 & -50 & 50150.07 & 61657.69 & 22 & -24 & 50199.27 & 61742.97 \\
\hline 8 & 0 & 50153.35 & 61821.69 & 22 & -25 & 50199.27 & 61739.69 \\
\hline 8 & -1 & 50153.35 & 61818.41 & 22 & -26 & 50199.27 & 61736.41 \\
\hline 8 & -2 & 50153.35 & 61815.13 & 22 & -27 & 50199.27 & 61733.13 \\
\hline 8 & -3 & 50153.35 & 61811.85 & 22 & -28 & 50199.27 & 61729.85 \\
\hline 8 & -4 & 50153.35 & 61808.57 & 22 & -29 & 50199.27 & 61726.57 \\
\hline 8 & -5 & 50153.35 & 61805.29 & 22 & -30 & 50199.27 & 61723.29 \\
\hline 8 & -6 & 50153.35 & 61802.01 & 22 & -31 & 50199.27 & 61720.01 \\
\hline 8 & -7 & 50153.35 & 61798.73 & 23 & -1 & 50202.55 & 61818.41 \\
\hline 8 & -8 & 50153.35 & 61795.45 & 23 & -2 & $50202.5 \dot{5}$ & 61815.13 \\
\hline 8 & -9 & 50153.35 & 61792.17 & 23 & -3 & 50202.55 & 61811.85 \\
\hline 8 & -10 & 50153.35 & 61788.89 & 23 & -4 & 50202.55 & 61808.57 \\
\hline 8 & -11 & 50153.35 & 61785.61 & 23 & -5 & 50202.55 & 61805.29 \\
\hline 8 & -12 & 50153.35 & 61782.33 & 23 & -6 & 50202.55 & 61802.01 \\
\hline 8 & -13 & 50153.35 & 61779.05 & 23 & -7 & 50202.55 & 61798.73 \\
\hline 8 & -14 & 50153.35 & 61775.77 & 23 & -8 & 50202.55 & 61795.45 \\
\hline 8 & -15 & 50153.35 & 61772.49 & 23 & -9 & 50202.55 & 61792.17 \\
\hline 8 & -16 & 50153.35 & 61769.21 & 23 & -10 & 50202.55 & 61788.89 \\
\hline 8 & -17 & 50153.35 & 61765.93 & 23 & -11 & 50202.55 & 61785.61 \\
\hline 8 & -18 & 50153.35 & 61762.65 & 23 & -12 & 50202.55 & 61782.33 \\
\hline 8 & -19 & 50153.35 & 61759.37 & 23 & -13 & 50202.55 & 61779.05 \\
\hline 8 & -20 & 50153.35 & 61756.09 & 23 & -14 & 50202.55 & 61775.77 \\
\hline 8 & -21 & 50153.35 & 61752.81 & 23 & -15 & 50202.55 & 61772.49 \\
\hline 8 & -22 & 50153.35 & 61749.53 & 23 & -16 & 50202.55 & 61769.21 \\
\hline 8 & -23 & 50153.35 & 61746.25 & 23 & -17 & 50202.55 & 61765.93 \\
\hline
\end{tabular}


Hydroflouric Acid Spill Magnetic Survey Coordinate Transformatiom

\begin{tabular}{|c|c|c|c|c|c|c|c|}
\hline \multicolumn{2}{|c|}{ GRID } & \multicolumn{2}{|c|}{ SITE } & \multicolumn{2}{|c|}{ GRID } & \multicolumn{2}{|c|}{ SITE } \\
\hline$x$ & $Y$ & EASTING & NORTHING & $x$ & $Y$ & EASTING & NORTHING \\
\hline 8 & -24 & 50153.35 & 61742.97 & 23 & -18 & 50202.55 & 61762.65 \\
\hline 8 & -25 & 50153.35 & 61739.69 & 23 & -19 & 50202.55 & 61759.37 \\
\hline 8 & -26 & 50153.35 & 61736.41 & 23 & -20 & 50202.55 & 61756.09 \\
\hline 8 & -27 & 50153.35 & 61733.13 & 23 & -21 & 50202.55 & 61752.81 \\
\hline 8 & -28 & 50153.35 & 61729.85 & 23 & -22 & 50202.55 & 61749.53 \\
\hline 8 & -29 & 50153.35 & 61726.57 & 23 & -23 & 50202.55 & 61746.25 \\
\hline 8 & -30 & 50153.35 & 61723.29 & 23 & -24 & 50202.55 & 61742.97 \\
\hline 8 & -31 & 50153.35 & 61720.01 & 23 & -25 & 50202.55 & 61739.69 \\
\hline 8 & -32 & 50153.35 & 61716.73 & 23 & -26 & 50202.55 & 61736.41 \\
\hline 8 & -33 & 50153.35 & 61713.45 & 23 & -27 & 50202.55 & 61733.13 \\
\hline 8 & -34 & 50153.35 & 61710.17 & 23 & -28 & 50202.55 & 61729.85 \\
\hline 8 & -35 & 50153.35 & 61706.89 & 24 & -1 & 50205.83 & 61818.41 \\
\hline 8 & -36 & 50153.35 & 61703.61 & 24 & -2 & 50205.83 & 61815.13 \\
\hline 8 & -37 & 50153.35 & 61700.33 & 24 & -3 & 50205.83 & 61811.85 \\
\hline 8 & -38 & 50153.35 & 61697.05 & 24 & -4 & 50205.83 & 61808.57 \\
\hline 8 & -39 & 50153.35 & 61693.77 & 24 & -5 & 50205.83 & 61805.29 \\
\hline 8 & -40 & 50153.35 & 61690.49 & 24 & -6 & 50205.83 & 61802.01 \\
\hline 8 & -41 & 50153.35 & 61687.21 & 24 & -7 & 50205.83 & 61798.73 \\
\hline 8 & -42 & 50153.35 & 61683.93 & 24 & -8 & 50205.83 & 61795.45 \\
\hline 8 & -43 & 50153.35 & 61680.65 & 24 & -9 & 50205.83 & 61792.17 \\
\hline 8 & -44 & 50153.35 & 61677.37 & 24 & -10 & 50205.83 & 61788.89 \\
\hline 8 & -45 & 50153.35 & 61674.09 & 24 & -11 & 50205.83 & 61785.61 \\
\hline 8 & -46 & 50153.35 & 61670.81 & 24 & -12 & 50205.83 & 61782.33 \\
\hline 8 & -47 & 50153.35 & 61667.53 & 24 & -13 & 50205.83 & 61779.05 \\
\hline 8 & -48 & 50153.35 & 61664.25 & 24 & -14 & 50205.83 & 61775.77 \\
\hline 8 & -49 & 50153.35 & 61660.97 & 24 & -15 & 50205.83 & 61772.49 \\
\hline 9 & 0 & 50156.63 & 61821.69 & 24 & -16 & 50205.83 & 61769.21 \\
\hline 9 & -1 & 50156.63 & 61818.41 & 24 & -17 & 50205.83 & 61765.93 \\
\hline 9 & -2 & 50156.63 & 61815.13 & 24 & -18 & 50205.83 & 61762.65 \\
\hline 9 & -3 & 50156.63 & 61811.85 & 24 & -19 & 50205.83 & 61759.37 \\
\hline 9 & -4 & 50156.63 & 61808.57 & 24 & -20 & 50205.83 & 61756.09 \\
\hline 9 & -5 & 50156.63 & 61805.29 & 24 & -21 & 50205.83 & 61752.81 \\
\hline 9 & -6 & 50156.63 & 61802.01 & 24 & -22 & 50205.83 & 61749.53 \\
\hline 9 & -7 & 50156.63 & 61798.73 & 24 & -23 & 50205.83 & 61746.25 \\
\hline 9 & -8 & 50156.63 & 61795.45 & 24 & -24 & 50205.83 & 61742.97 \\
\hline 9 & -9 & 50156.63 & 61792.17 & 24 & -25 & 50205.83 & 61739.69 \\
\hline 9 & -10 & 50156.63 & 61788.89 & 24 & -26 & 50205.83 & 61736.41 \\
\hline 9 & -11 & 50156.63 & 61785.61 & 24 & -27 & 50205.83 & 61733.13 \\
\hline 9 & -12 & 50156.63 & 61782.33 & 24 & -28 & 50205.83 & 61729.85 \\
\hline 9 & -13 & 50156.63 & 61779.05 & 25 & -4 & 50209.11 & 61808.57 \\
\hline 9 & -14 & 50156.63 & 61775.77 & 25 & -5 & 50209.11 & 61805.29 \\
\hline 9 & -15 & 50156.63 & 61772.49 & 25 & -6 & 50209.11 & 61802.01 \\
\hline 9 & -16 & 50156.63 & 61769.21 & 25 & -7 & 50209.11 & 61798.73 \\
\hline 9 & -17 & 50156.63 & 61765.93 & 25 & -8 & 50209.11 & 61795.45 \\
\hline 9 & -18 & 50156.63 & 61762.65 & 25 & -9 & 50209.11 & 61792.17 \\
\hline 9 & -19 & 50156.63 & 61759.37 & 25 & -10 & 50209.11 & 61788.89 \\
\hline 9 & -20 & 50156.63 & 61756.09 & 25 & -11 & 50209.11 & 61785.61 \\
\hline 9 & -21 & 50156.63 & 61752.81 & 25 & -12 & 50209.11 & 61782.33 \\
\hline 9 & -22 & 50156.63 & 61749.53 & 25 & -13 & 50209.11 & 61779.05 \\
\hline
\end{tabular}


Hydroflouric Acid Spill Magnetic Survey Coordinate Transformatiom

\begin{tabular}{|c|c|c|c|c|c|c|c|}
\hline \multicolumn{2}{|c|}{ GRID } & \multicolumn{2}{|c|}{ SITE } & \multicolumn{2}{|c|}{ GRID } & \multicolumn{2}{|c|}{ SITE } \\
\hline$x$ & $Y$ & EASTING & NORTHING & $\mathbf{x}$ & $\mathbf{Y}$ & EASTING & NORTHING \\
\hline 9 & -23 & 50156.63 & 61746.25 & 25 & -14 & 50209.11 & 61775.77 \\
\hline 9 & -24 & 50156.63 & 61742.97 & 25 & -15 & 50209.11 & 61772.49 \\
\hline 9 & -25 & 50156.63 & 61739.69 & 25 & -16 & 50209.11 & 61769.21 \\
\hline 9 & -26 & 50156.63 & 61736.41 & 25 & -17 & 50209.11 & 61765.93 \\
\hline 9 & -27 & 50156.63 & 61733.13 & 25 & -18 & 50209.11 & 61762.65 \\
\hline 9 & -28 & 50156.63 & 61729.85 & 25 & -19 & 50209.11 & 61759.37 \\
\hline 9 & -29 & 50156.63 & 61726.57 & 25 & -20 & 50209.11 & 61756.09 \\
\hline 9 & -30 & 50156.63 & 61723.29 & 25 & -21 & 50209.11 & 61752.81 \\
\hline 9 & -31 & 50156.63 & 61720.01 & 25 & -22 & 50209.11 & 61749.53 \\
\hline 9 & -32 & 50156.63 & 61716.73 & 25 & -23 & 50209.11 & 61746.25 \\
\hline 9 & -33 & 50156.63 & 61713.45 & 25 & -24 & 50209.11 & 61742.97 \\
\hline 9 & -34 & 50156.63 & 61710.17 & 25 & -25 & 50209.11 & 61739.69 \\
\hline 9 & -35 & 50156.63 & 61706.89 & 25 & -26 & 50209.11 & 61736.41 \\
\hline 9 & -36 & 50156.63 & 61703.61 & 25 & -27 & 50209.11 & 61733.13 \\
\hline 9 & -37 & 50156.63 & 61700.33 & 25 & -28 & 50209.11 & 61729.85 \\
\hline 9 & -38 & 50156.63 & 61697.05 & 26 & -5.5 & 50212.39 & 61803.65 \\
\hline 9 & -39 & 50156.63 & 61693.77 & 26 & -6.5 & 50212.39 & 61800.37 \\
\hline 9 & -40 & 50156.63 & 61690.49 & 26 & -7.5 & 50212.39 & 61797.09 \\
\hline 9 & -41 & 50156.63 & 61687.21 & 26 & -8.5 & 50212.39 & 61793.81 \\
\hline 9 & -42 & 50156.63 & 61683.93 & 26 & -9.5 & 50212.39 & 61790.53 \\
\hline 9 & -43 & 50156.63 & 61680.65 & 26 & -10.5 & 50212.39 & 61787.25 \\
\hline 9 & -44 & 50156.63 & 61677.37 & 26 & -11.5 & 50212.39 & 61783.97 \\
\hline 9 & -45 & 50156.63 & 61674.09 & 26 & -12.5 & 50212.39 & 61780.69 \\
\hline 9 & -46 & 50156.63 & 61670.81 & 26 & -13.5 & 50212.39 & 61777.41 \\
\hline 9 & -47 & 50156.63 & 61667.53 & 26 & -14.5 & 50212.39 & 61774.13 \\
\hline 10 & 0 & 50159.91 & 61821.69 & 26 & -15.5 & 50212.39 & 61770.85 \\
\hline 10 & -1 & 50159.91 & 61818.41 & 26 & -16.5 & 50212.39 & 61767.57 \\
\hline 10 & -2 & 50159.91 & 61815.13 & 26 & -17.5 & 50212.39 & 61764.29 \\
\hline 10 & -3 & 50159.91 & 61811.85 & 26 & -18.5 & 50212.39 & 61761.01 \\
\hline 10 & -4 & 50159.91 & 61808.57 & 26 & -19.5 & 50212.39 & 61757.73 \\
\hline 10 & -5 & 50159.91 & 61805.29 & 26 & -20.5 & 50212.39 & 61754.45 \\
\hline 10 & -6 & 50159.91 & 61802.01 & 26 & -21.5 & 50212.39 & 61751.17 \\
\hline 10 & -7 & 50159.91 & 61798.73 & 26 & -22.5 & 50212.39 & 61747.89 \\
\hline 10 & -8 & 50159.91 & 61795.45 & 26 & -23.5 & 50212.39 & 61744.61 \\
\hline 10 & -9 & 50159.91 & 61792.17 & 26 & -24.5 & 50212.39 & 61741.33 \\
\hline 10 & -10 & 50159.91 & 61788.89 & 26 & -25.5 & 50212.39 & 61738.05 \\
\hline 10 & -11 & 50159.91 & 61785.61 & 26 & -26.5 & 50212.39 & 61734.77 \\
\hline 10 & -12 & 50159.91 & 61782.33 & 27 & -6.5 & 50215.67 & 61800.37 \\
\hline 10 & -13 & 50159.91 & 61779.05 & 27 & -7.5 & 50215.67 & 61797.09 \\
\hline 10 & -14 & 50159.91 & 61775.77 & 27 & -8.5 & 50215.67 & 61793.81 \\
\hline 10 & -15 & 50159.91 & 61772.49 & 27 & -9.5 & 50215.67 & 61790.53 \\
\hline 10 & -16 & 50159.91 & 61769.21 & 27 & -10.5 & 50215.67 & 61787.25 \\
\hline 10 & -17 & 50159.91 & 61765.93 & 27 & -11.5 & 50215.67 & 61783.97 \\
\hline 10 & -18 & 50159.91 & 61762.65 & 27 & -12.5 & 50215.67 & 61780.69 \\
\hline 10 & -19 & 50159.91 & 61759.37 & 27 & -13.5 & 50215.67 & 61777.41 \\
\hline 10 & -20 & 50159.91 & 61756.09 & 27 & -14.5 & 50215.67 & 61774.13 \\
\hline 10 & -21 & 50159.91 & 61752.81 & 27 & -15.5 & 50215.67 & 61770.85 \\
\hline 10 & -22 & 50159.91 & 61749.53 & 27 & -16.5 & 50215.67 & 61767.57 \\
\hline 10 & -23 & 50159.91 & 61746.25 & 27 & -17.5 & 50215.67 & 61764.29 \\
\hline
\end{tabular}


Hydroflouric Acid Spill Magnetic Survey Coordinate Transformatiom

\begin{tabular}{|c|c|c|c|c|c|c|c|}
\hline \multicolumn{2}{|c|}{ GRID } & \multicolumn{2}{|c|}{ SITE } & \multicolumn{2}{|c|}{ GRID } & \multicolumn{2}{|c|}{ SITE } \\
\hline $\mathbf{x}$ & $\mathbf{Y}$ & EASTING & NORTHING & $\mathbf{x}$ & $Y$ & EASTING & NORTHING \\
\hline 10 & -24 & 50159.91 & 61742.97 & 27 & -18.5 & 50215.67 & 61761.01 \\
\hline 10 & -25 & 50159.91 & 61739.69 & 27 & -19.5 & 50215.67 & 61757.73 \\
\hline 10 & -26 & 50159.91 & 61736.41 & 27 & -20.5 & 50215.67 & 61754.45 \\
\hline 10 & -27 & 50159.91 & 61733.13 & 27 & -21.5 & 50215.67 & 61751.17 \\
\hline 10 & -28 & 50159.91 & 61729.85 & 27 & -22.5 & 50215.67 & 61747.89 \\
\hline 10 & -29 & 50159.91 & 61726.57 & 27 & -23.5 & $5021 \widehat{\widehat{c}} .67$ & 61744.61 \\
\hline 10 & -30 & 50159.91 & 61723.29 & 27 & -24.5 & 50215.67 & 61741.33 \\
\hline 10 & -31 & 50159.91 & 61720.01 & 27 & -25.5 & 50215.67 & 61738.05 \\
\hline 10 & -32 & 50159.91 & 61716.73 & 28 & -8.25 & 50218.95 & 61794.63 \\
\hline 10 & -33 & 50159.91 & 61713.45 & 28 & -9.25 & 50218.95 & 61791.35 \\
\hline 10 & -34 & 50159.91 & 61710.17 & 28 & -10.25 & 50218.95 & 61788.07 \\
\hline 10 & -35 & 50159.91 & 61706.89 & 28 & -11.25 & 50218.95 & 61784.79 \\
\hline 10 & -36 & 50159.91 & 61703.61 & 28 & -12.25 & 50218.95 & 61781.51 \\
\hline 10 & -37 & 50159.91 & 61700.33 & 28 & -13.25 & 50218.95 & 61778.23 \\
\hline 10 & -38 & 50159.91 & 61697.05 & 28 & -14.25 & 50218.95 & 61774.95 \\
\hline 10 & -39 & 50159.91 & 61693.77 & 28 & -15.25 & 50218.95 & 61771.67 \\
\hline 10 & -40 & 50159.91 & $61690: 49$ & 28 & -16.25 & 50218.95 & 61768.39 \\
\hline 10 & -41 & 50159.91 & 61687.21 & 28 & -17.25 & 50218.95 & 61765.11 \\
\hline 10 & -42 & 50159.91 & 61683.93 & 28 & -18.25 & 50218.95 & 61761.83 \\
\hline 10 & -43 & 50159.91 & 61680.65 & 28 & -19.25 & 50218.95 & 61758.55 \\
\hline 10 & -44 & 50159.91 & 61677.37 & 28 & -20.25 & 50218.95 & 61755.27 \\
\hline 10 & -45 & 50159.91 & 61674.09 & 28 & -21.25 & 50218.95 & 61751.99 \\
\hline 10 & -46 & 50159.91 & 61670.81 & 28 & -22.25 & 50218.95 & 61748.71 \\
\hline 11 & 0 & 50163.19 & 61821.69 & 28 & -23.25 & 50218.95 & 61745.43 \\
\hline 11 & -1 & 50163.19 & 61818.41 & 28 & -24.25 & 50218.95 & 61742.15 \\
\hline 11 & -2 & 50163.19 & 61815.13 & 29 & -10 & 50222.23 & 61788.89 \\
\hline 11 & -3 & 50163.19 & 61811.85 & 29 & -11 & 50222.23 & 61785.61 \\
\hline 11 & -4 & 50163.19 & 61808.57 & 29 & -12 & 50222.23 & 61782.33 \\
\hline 11 & -5 & 50163.19 & 61805.29 & 29 & -13 & 50222.23 & 61779.05 \\
\hline 11 & -6 & 50163.19 & 61802.01 & 29 & -14 & 50222.23 & 61775.77 \\
\hline 11 & -7 & 50163.19 & 61798.73 & 29 & -15 & 50222.23 & 61772.49 \\
\hline 11 & -8 & 50163.19 & 61795.45 & 29 & -16 & 50222.23 & 61769.21 \\
\hline 11 & -9 & 50163.19 & 61792.17 & 29 & -17 & 50222.23 & 61765.93 \\
\hline 11 & -10 & 50163.19 & 61788.89 & 29 & -18 & 50222.23 & 61762.65 \\
\hline 11 & -11 & 50163.19 & 61785.61 & 29 & -19 & 50222.23 & 61759.37 \\
\hline 11 & -12 & 50163.19 & 61782.33 & 29 & -20 & 50222.23 & 61756.09 \\
\hline 11 & -13 & 50163.19 & 61779.05 & 29 & -21 & 50222.23 & 61752.81 \\
\hline 11 & -14 & 50163.19 & 61775.77 & 29 & -22 & 50222.23 & 61749.53 \\
\hline 11 & -15 & 50163.19 & 61772.49 & 29 & -23 & 50222.23 & 61746.25 \\
\hline 11 & -16 & 50163.19 & 61769.21 & 30 & -15 & 50225.51 & 61772.49 \\
\hline 11 & -17 & 50163.19 & 61765.93 & 31 & -15 & 50225.51 & 61759.37 \\
\hline 11 & -18 & 50163.19 & 61762.65 & 32 & -15 & 50228.79 & 61772.49 \\
\hline 11 & -19 & 50163.19 & 61759.37 & 30 & -19 & 50228.79 & 61759.37 \\
\hline 11 & -20 & 50163.19 & 61756.09 & 31 & -19 & 50232.07 & 61772.49 \\
\hline 11 & -21 & 50163.19 & 61752.81 & 32 & -19 & 50232.07 & 61759.37 \\
\hline 11 & -22 & 50163.19 & 61749.53 & & & & \\
\hline 11 & -23 & 50163.19 & 61746.25 & & & & \\
\hline 11 & -24 & 50163.19 & 61742.97 & & & & \\
\hline & & & & & & $\begin{array}{c}\because \\
-\end{array}$ & \\
\hline & & & & & & $\because \quad$, & \\
\hline & & & & & & & \\
\hline
\end{tabular}


Hydroflouric Acid Spill Magnetic Survey

\section{FIGURE CAPTIONS}

Figure 1. Location of Hydroflouric Acid Spill Area on SRS.

Figure 2. Typical temporal variations exhibited by the total magnetic field due to diurnal variation, micropulsations, and magnetic storms on the sun (adapted from Breiner, 1973).

Figure 3. Superposition of the earth's ambient magnetic field and anomalous fields produced by induced monopoles and dipoles to produce anomalies in the total field (adapted from Breiner, 1973).

Figure 4. The relationship of magnetic inclination and declination to geographic directions on the earth (adapted from Telford and others, 1976).

Figure 5. Typical profiles of magnetic field intensity across a monopole and dipole for conditions similar to SRS. Also shown is a contour representation of magnetic field intensity over a dipole with accompanying representative profiles (adapted from Breiner, 1973).

Figure 6. Magnetic intensity profiles across several geometric shapes of interest for magnetic field inclination similar to that of SRS (i.e: 60 degrees) (adapted from Breiner, 1973).

Figure 7. The intensity of the total magnetic field compared with the vertical gradient over a prismatic body (adapted from Breiner, 1973).

Figure 8. Model total field and vertical gradient magnetic profiles for single 55 gallon steel drums in vertical position with the top at various distances below the sensor. Profiles calculated for a total field intensity of $53220 \mathrm{nT}$ and northern hemisphere inclination of 70 degrees (adapted from Gilkeson and others, 1990).

Figure 9. Contour representation of total field anomaly calculated for a single 55 gallon steel drum in vertical position with the top $10 \mathrm{ft}$ below the sensor. Calculation based on a total field intensity of $53220 \mathrm{nT}$ and northern hemisphere inclination of 70 degrees (adapted from Gilkeson and others, 1990). ' .

Figure 10. Contour representation of a cashe of 5 steel 55 gallon drums in a $12 \mathrm{ft}$ by $12 \mathrm{ft}$ area with the top of the drums $10 \mathrm{ft}$ below the sensor. Calculation based on a total field intensity of $53220 \mathrm{nT}$ and northern hemisphere inclination of 70 degrees (adapted from Gilkeson and others, 1990).

Figure 11. Planimetric map of the Hydroflouric Acid Spill Area. 
Hydroflouric Acid Spill Magnetic Survey

Figure 12. Station locations for the Hydroflouric Acid Spill Magnetic Survey. Station locations marked by "+"

Figure 13. Color contoured Hydroflouric Acid Spill Area magnetic survey diurnally corrected top sensor data.

Figure 14. Color contoured Hydroflouric Acid Spill Area magnetic survey diurnally corrected bottom sensor data.

Figure 15. Color contoured Hydrofluoric Acid Spill magnetic survey vertical gradient.

Figure 16. Locations of magnetic anomaly sources not associated with prominent features on the surface of the Hydroflouric Acid Spill Area. 


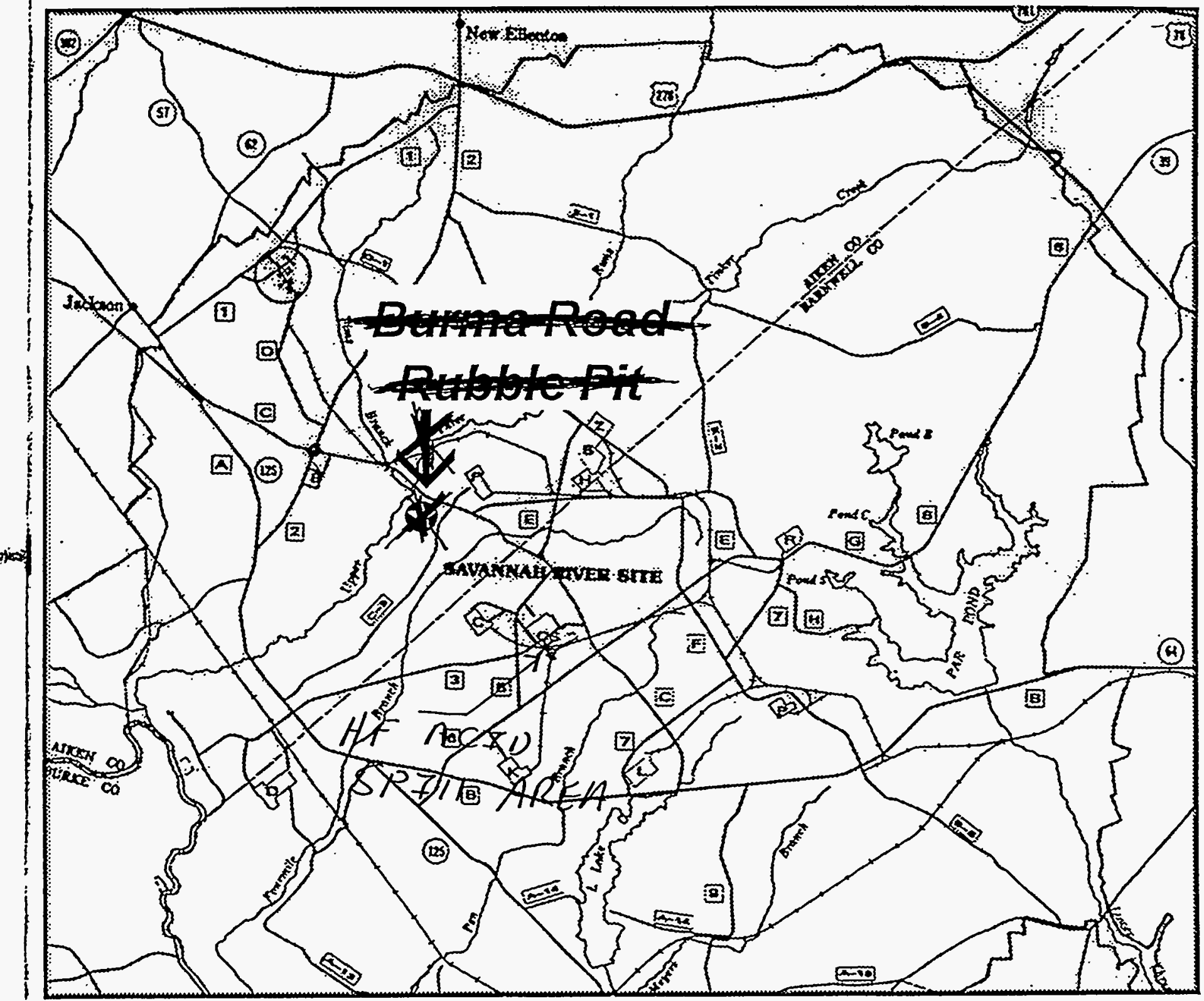

in Fig./ HF ACIO SPIII 


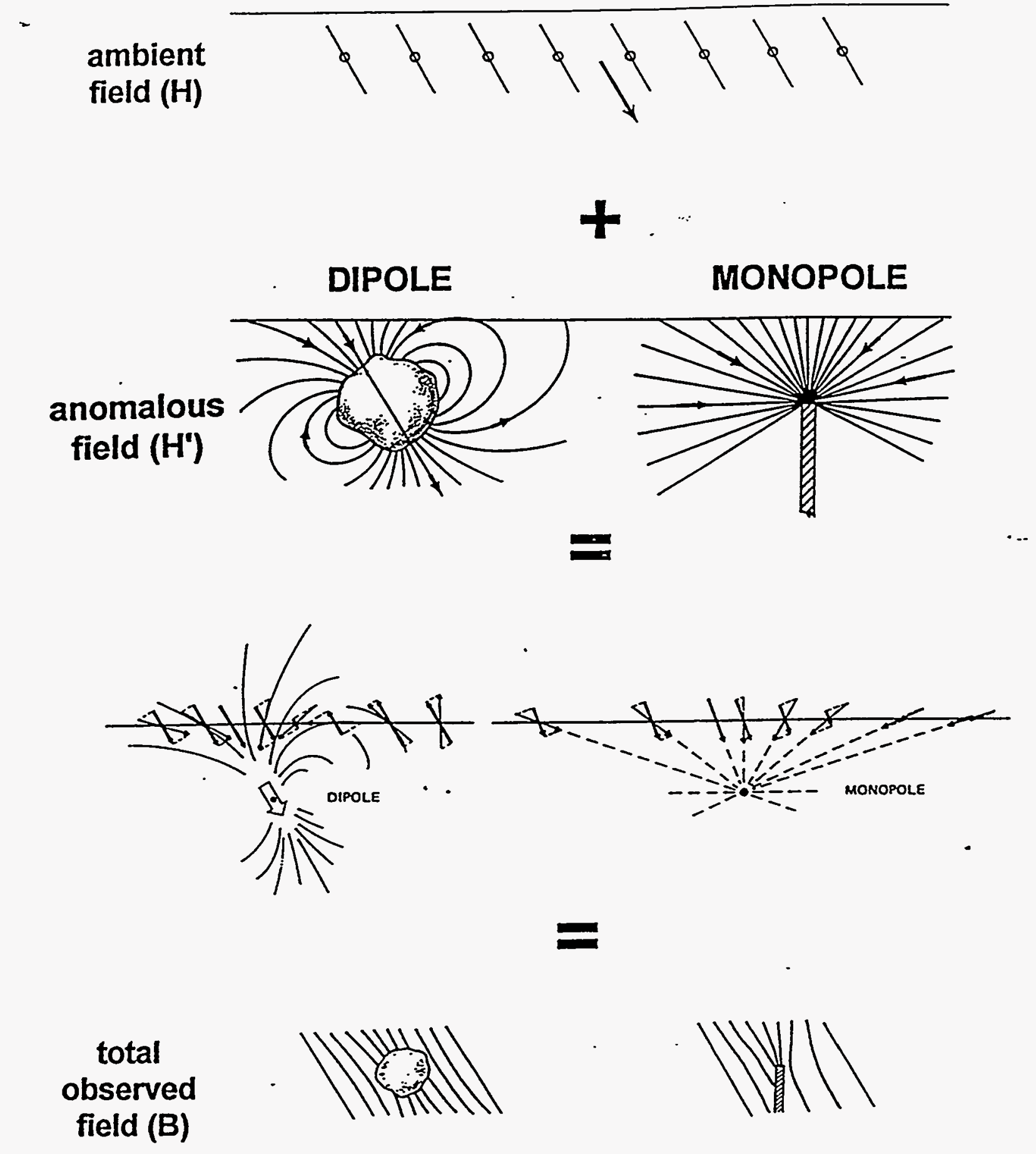

Yigure 3 


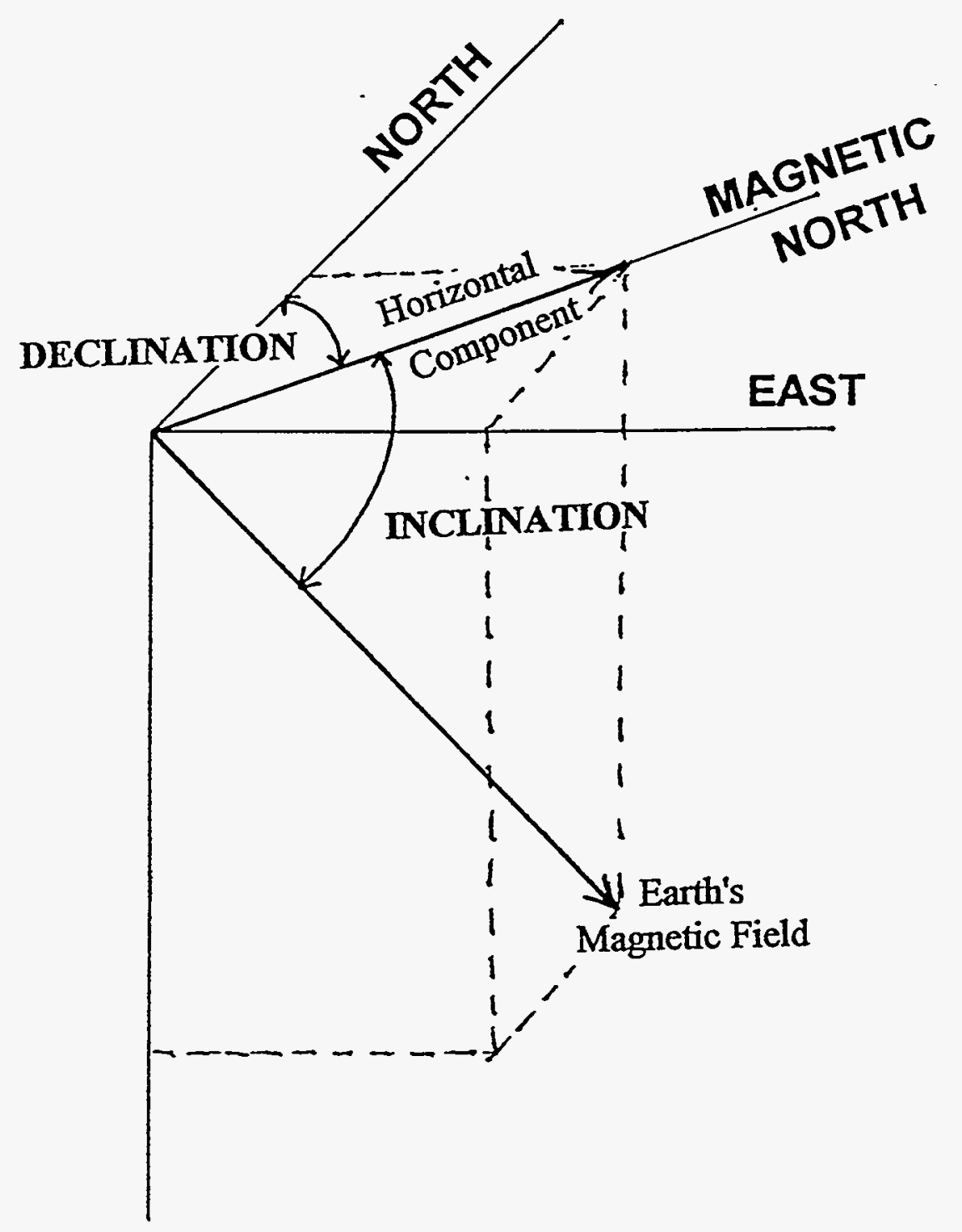

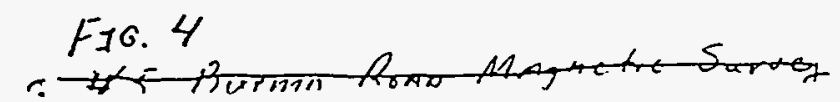



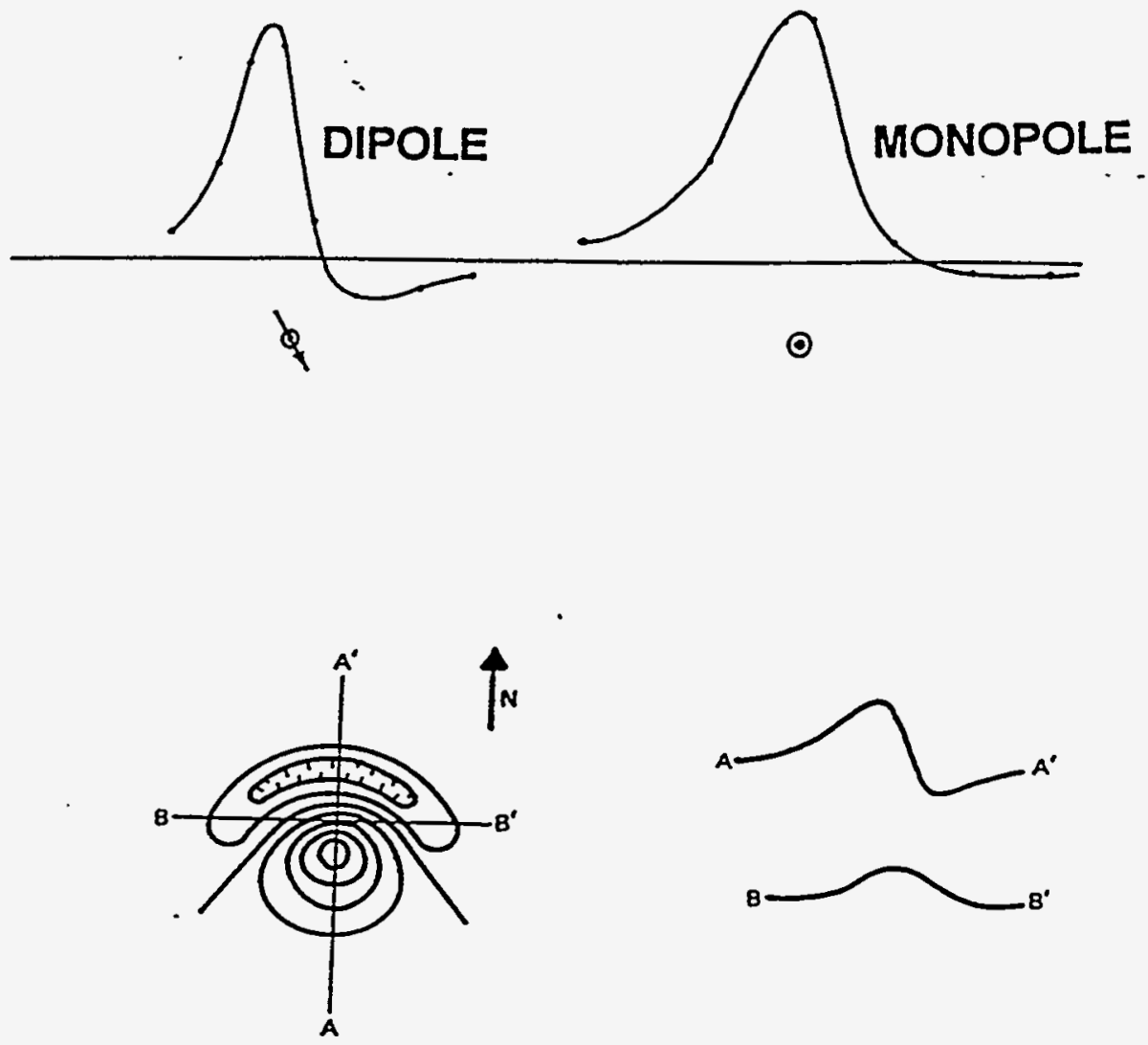

FIg. 5. HF ACID SPZLC

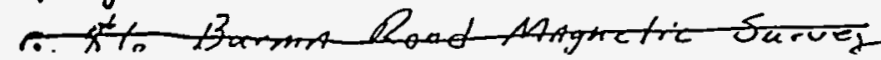




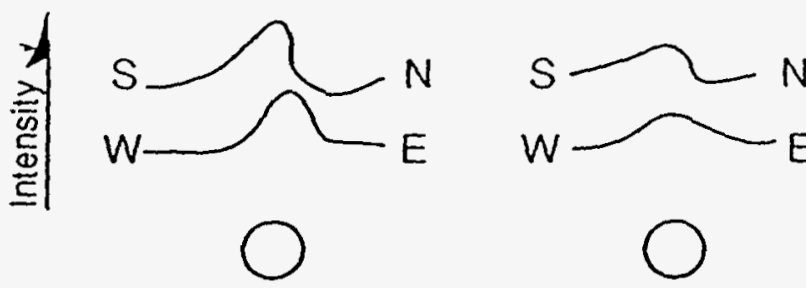

Sphere

Horizontal

Cylinder

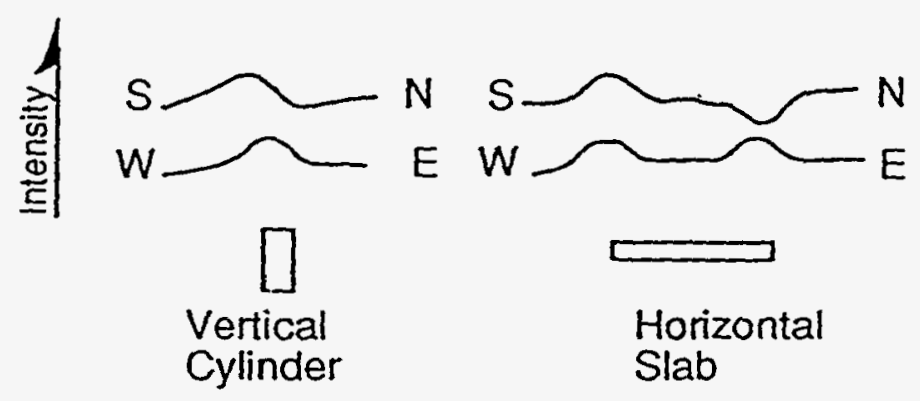

M93×2091.05.All

F76. 6

sirime:

Magnetic intensity profiles across several geometric shapes of interest for magnetic field inclination similar to that of SRS (i.e., 60 degrees) (adapted from Breiner, 1973).
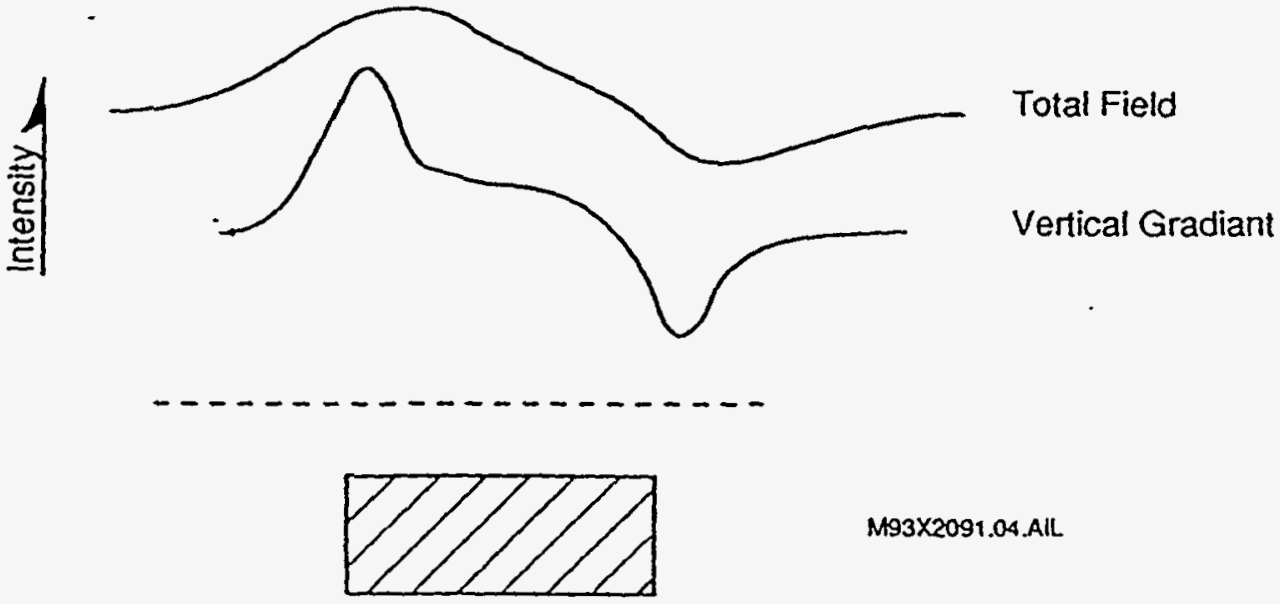

M93X2091.04.AlL

The intensity of the total magnetic field compared with the vertical gradient over a prismatic body (adapied from Breiner, 1973). 

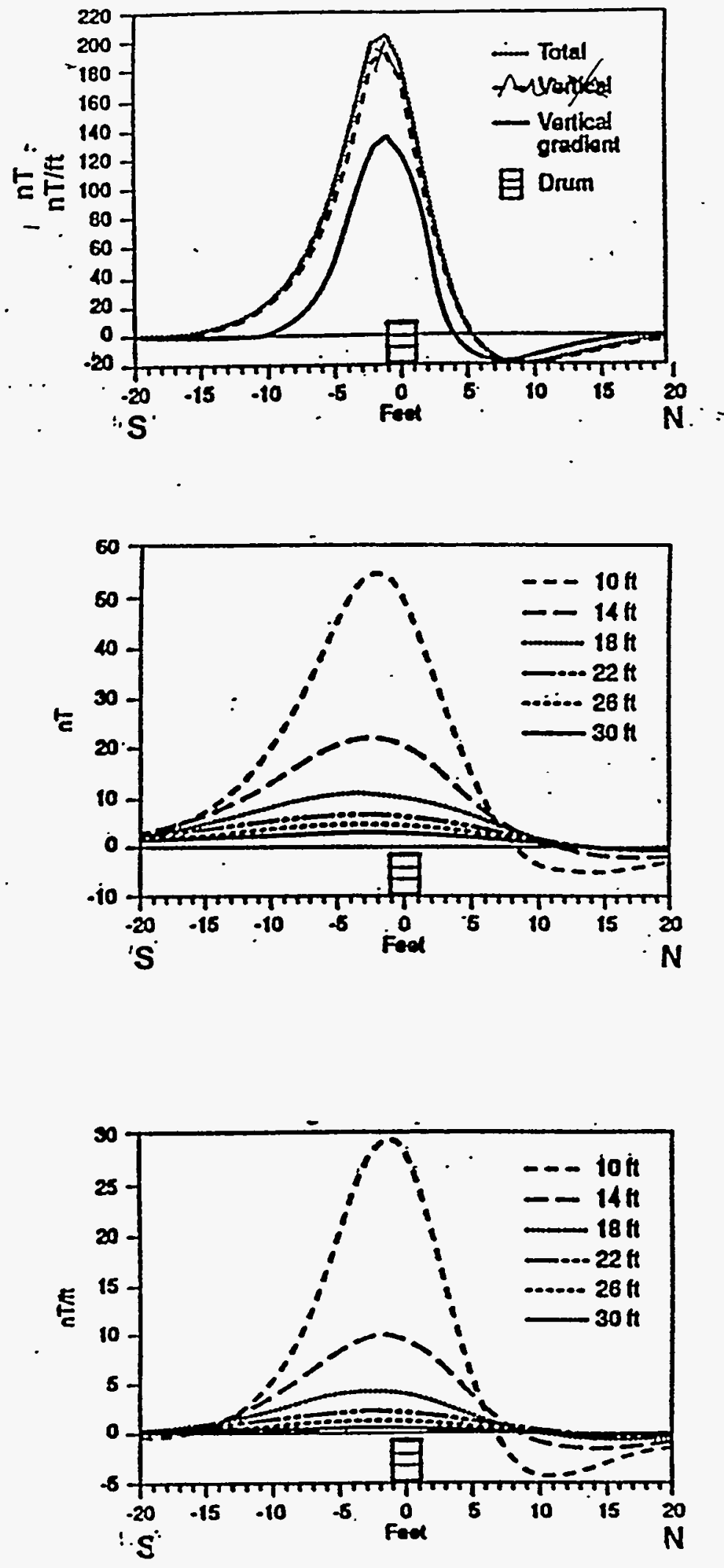

\section{STM \\ SINGLE DRUM}

$(6 \mathrm{ft})$
TOTAL FIELD SINGLE DRUM $(10-30 \mathrm{ft})$

\section{GRADIENT SINGLE DRUM $(10-30 \mathrm{ft})$}

FIG. 8 HF ACIN spill

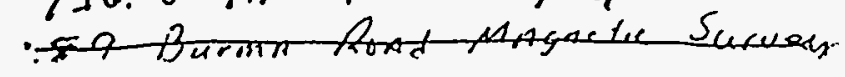




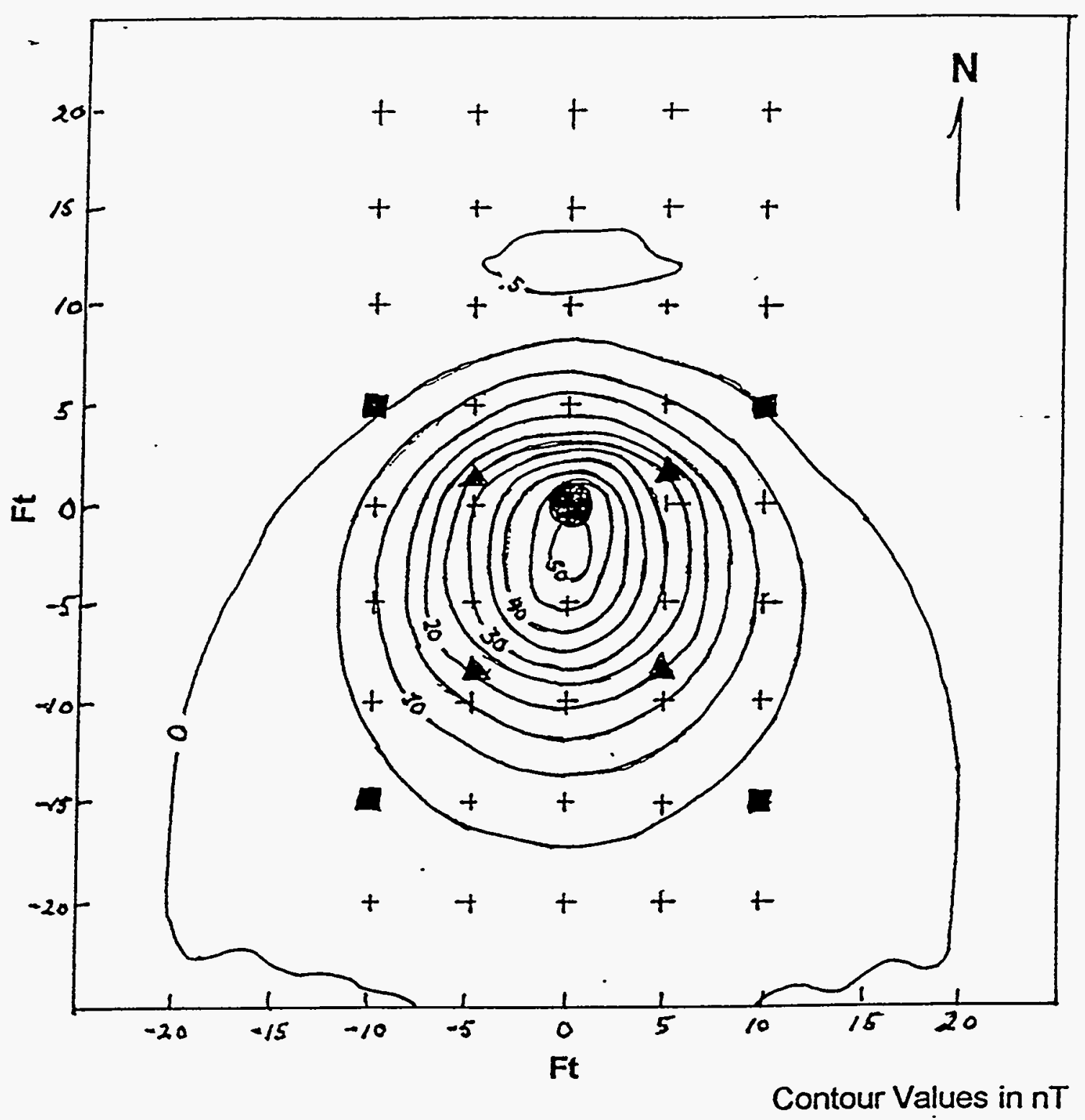

Drum Location

$+5 \mathrm{Ft}$ Grid Nodes

A 10 Ft Grid Nodes

20 Ft Grid Nodes

FT 6. 9 HF ACID SPILL 


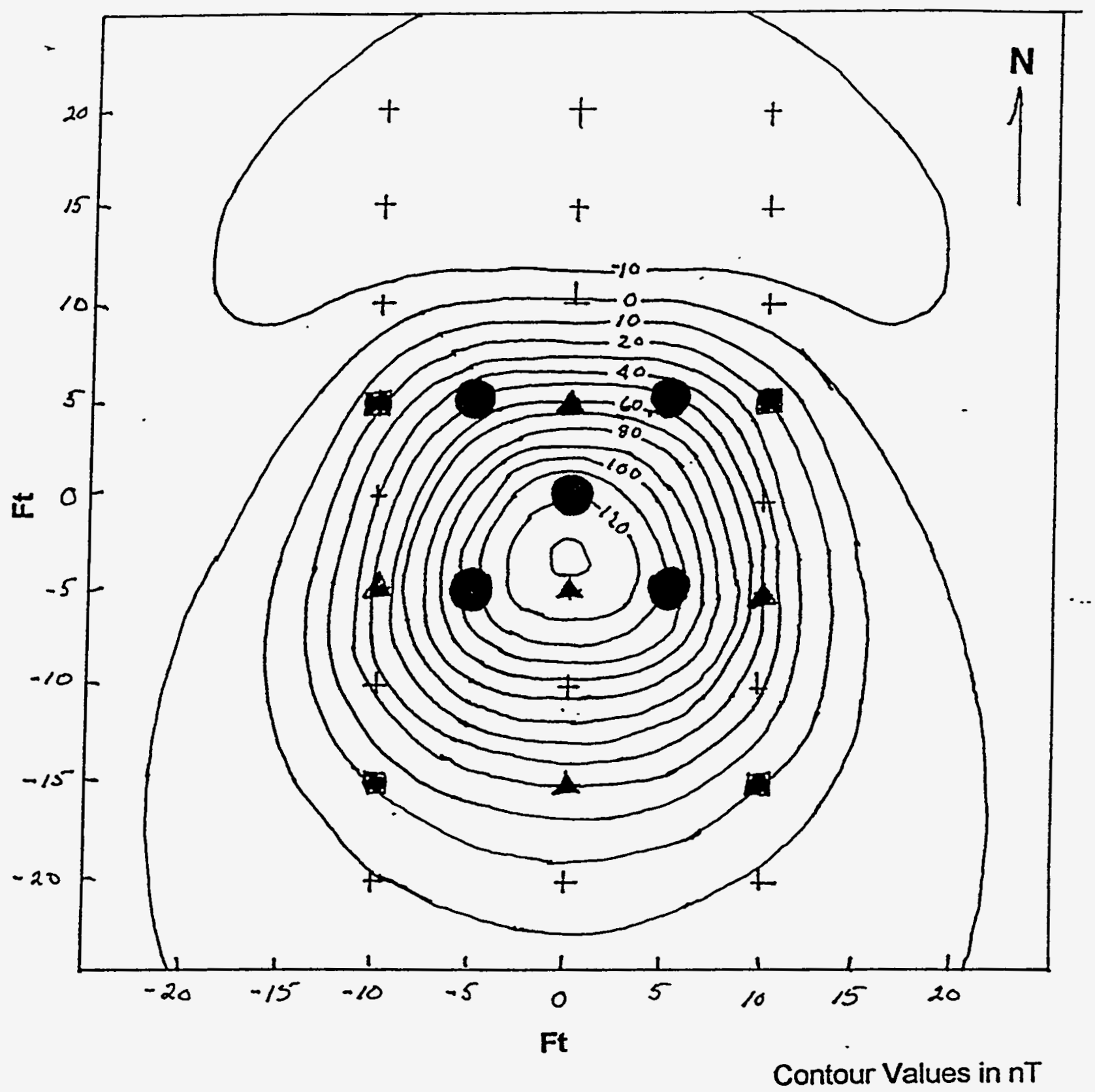

- Drum Location

$10 \mathrm{Ft}$ Grid Nodes
$20 \mathrm{Ft}$ Grid Nodes

FIG. 10 HF ACID SIILL

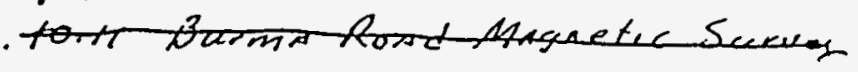




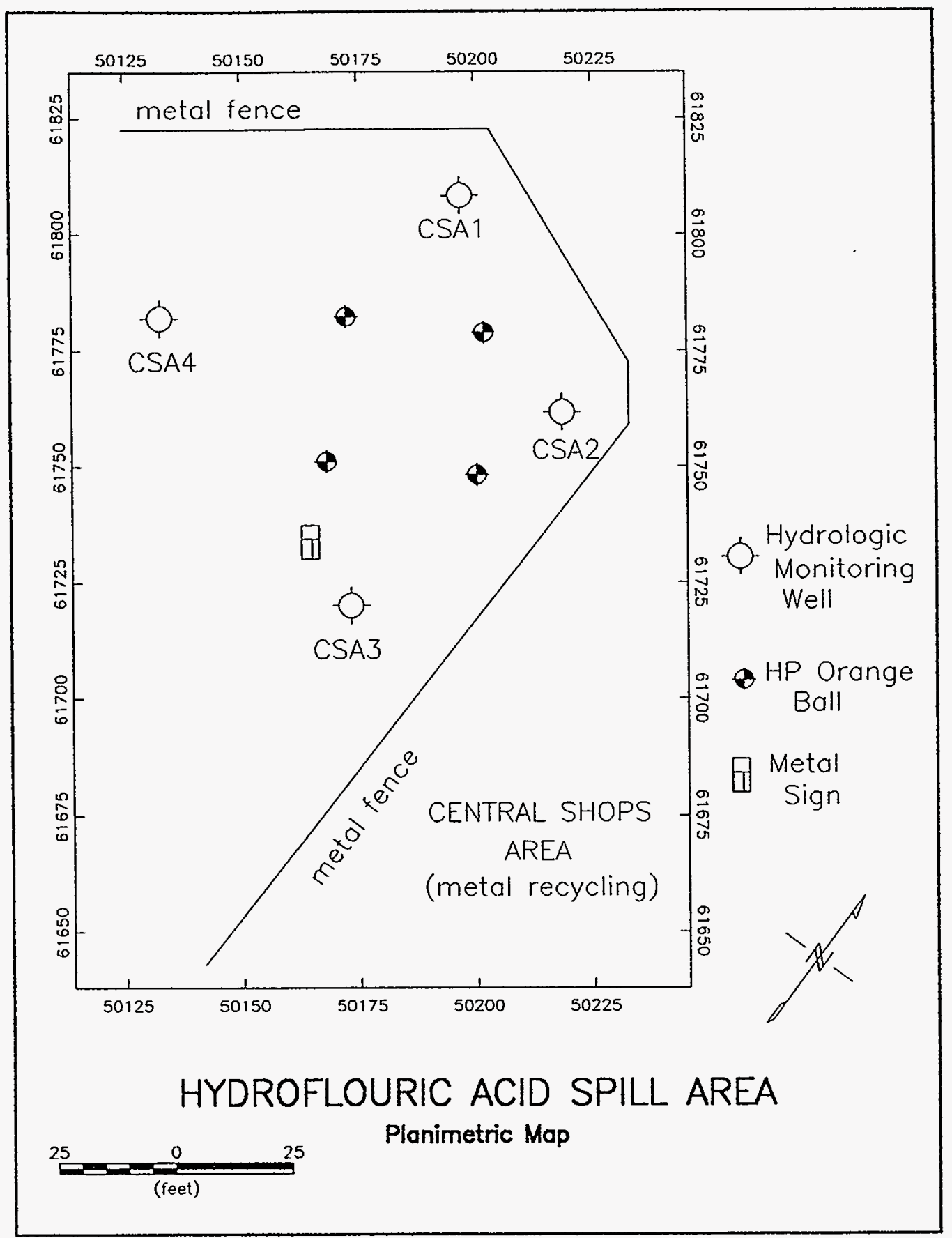




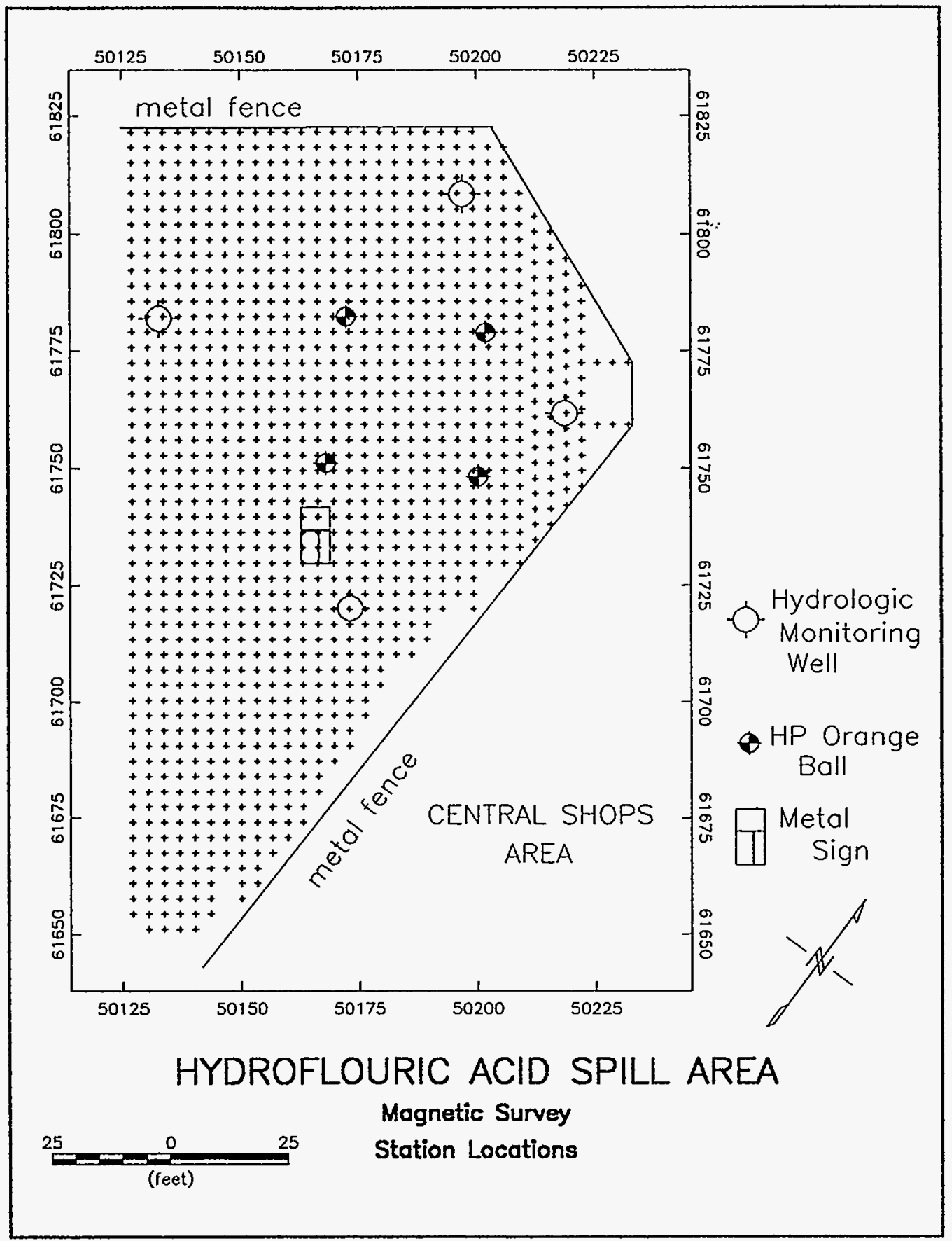




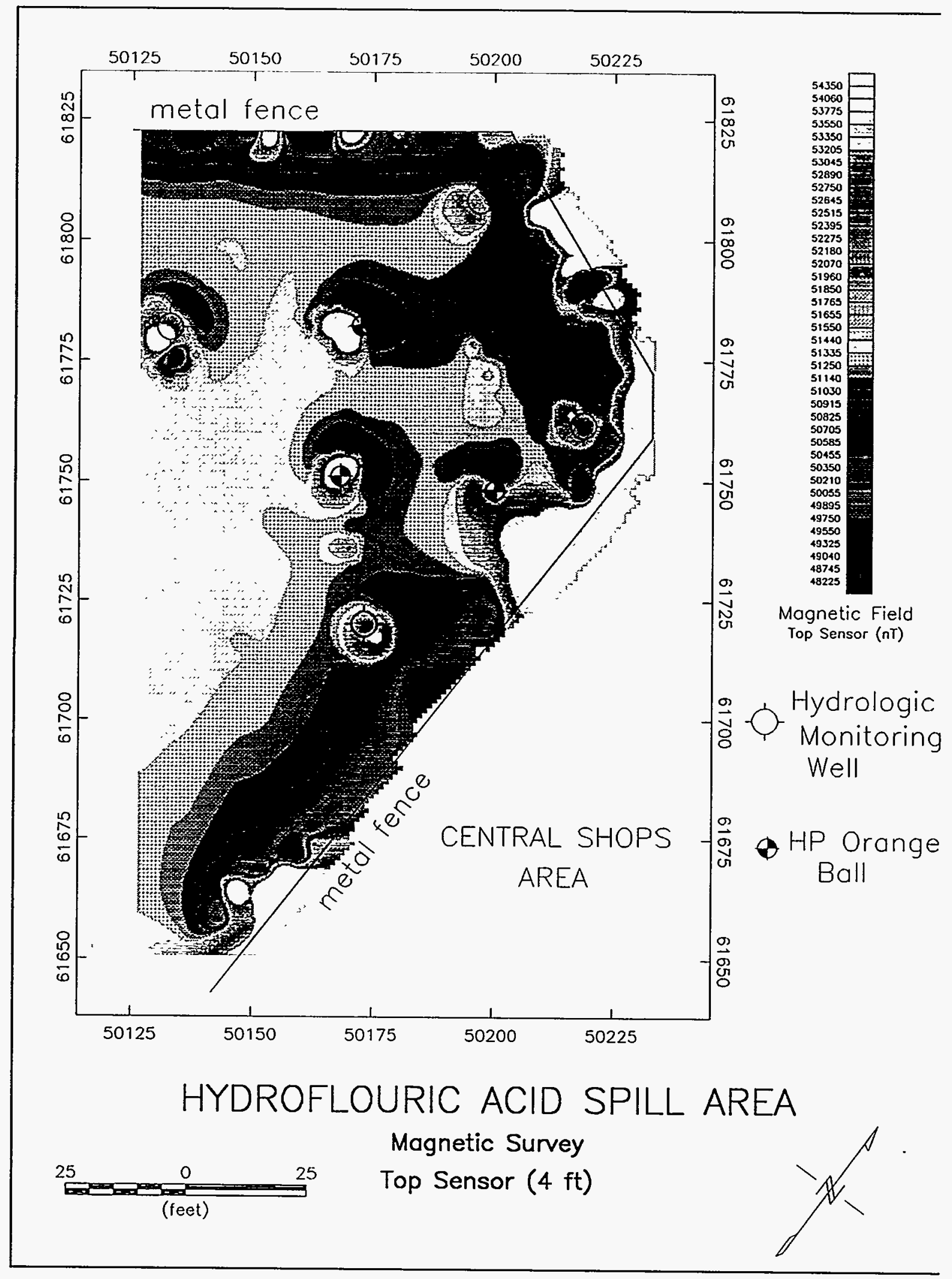




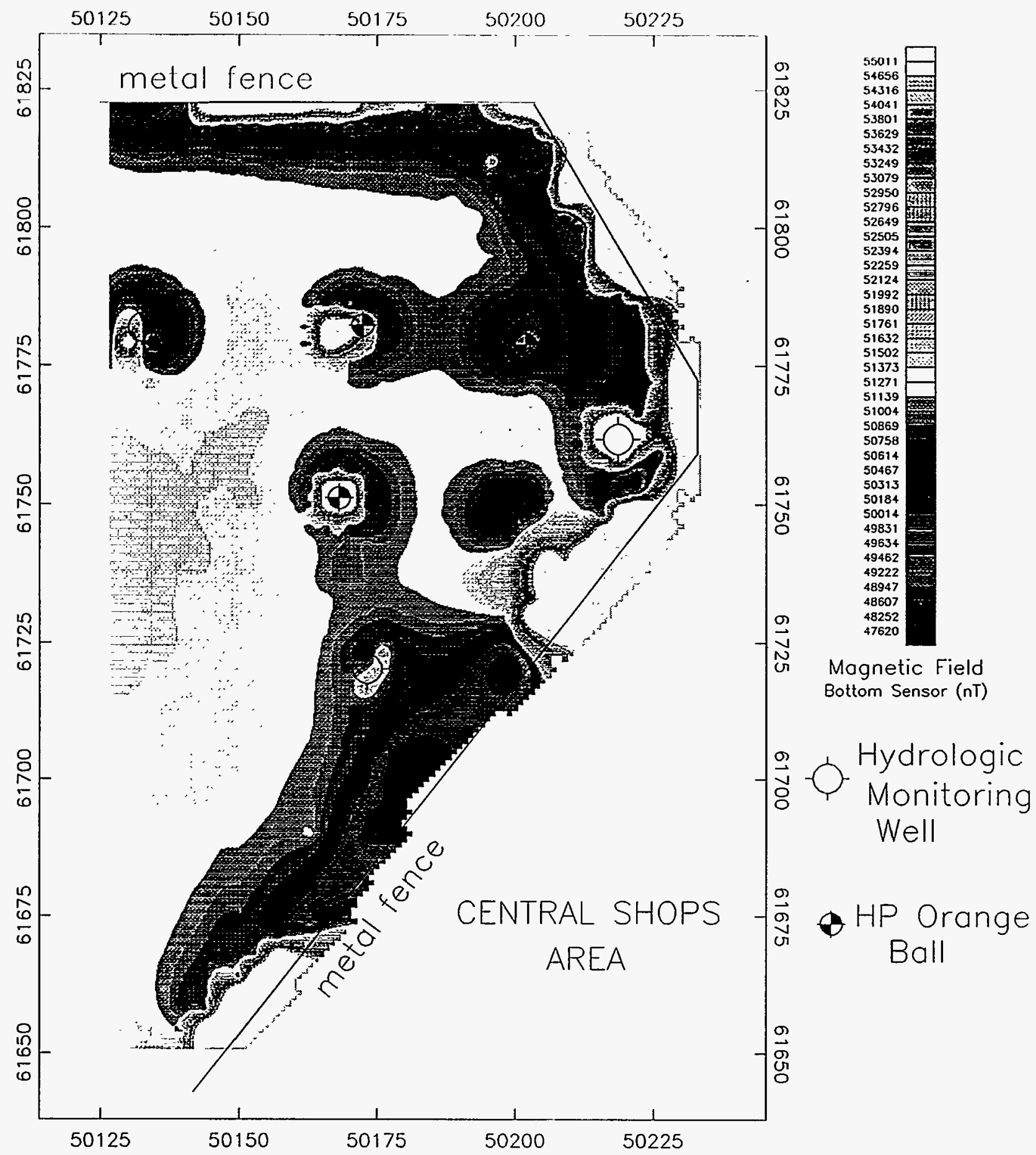

\section{HYDROFLOURIC ACID SPILL AREA}

Magnetic Survey
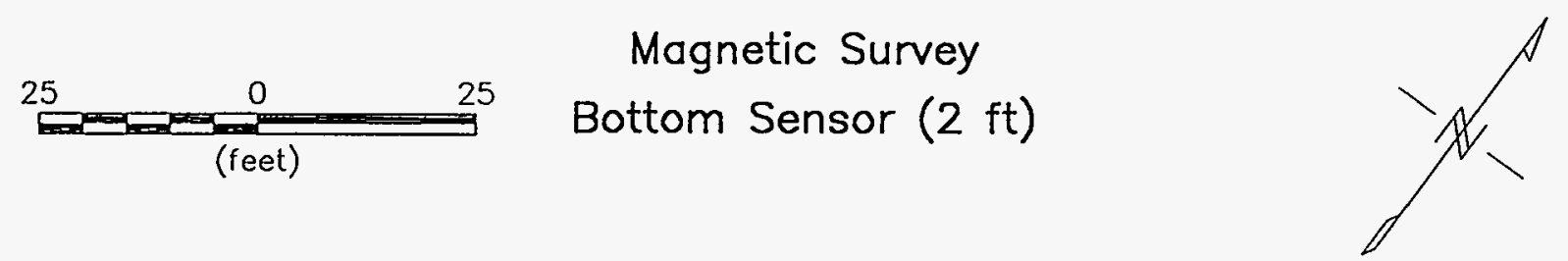


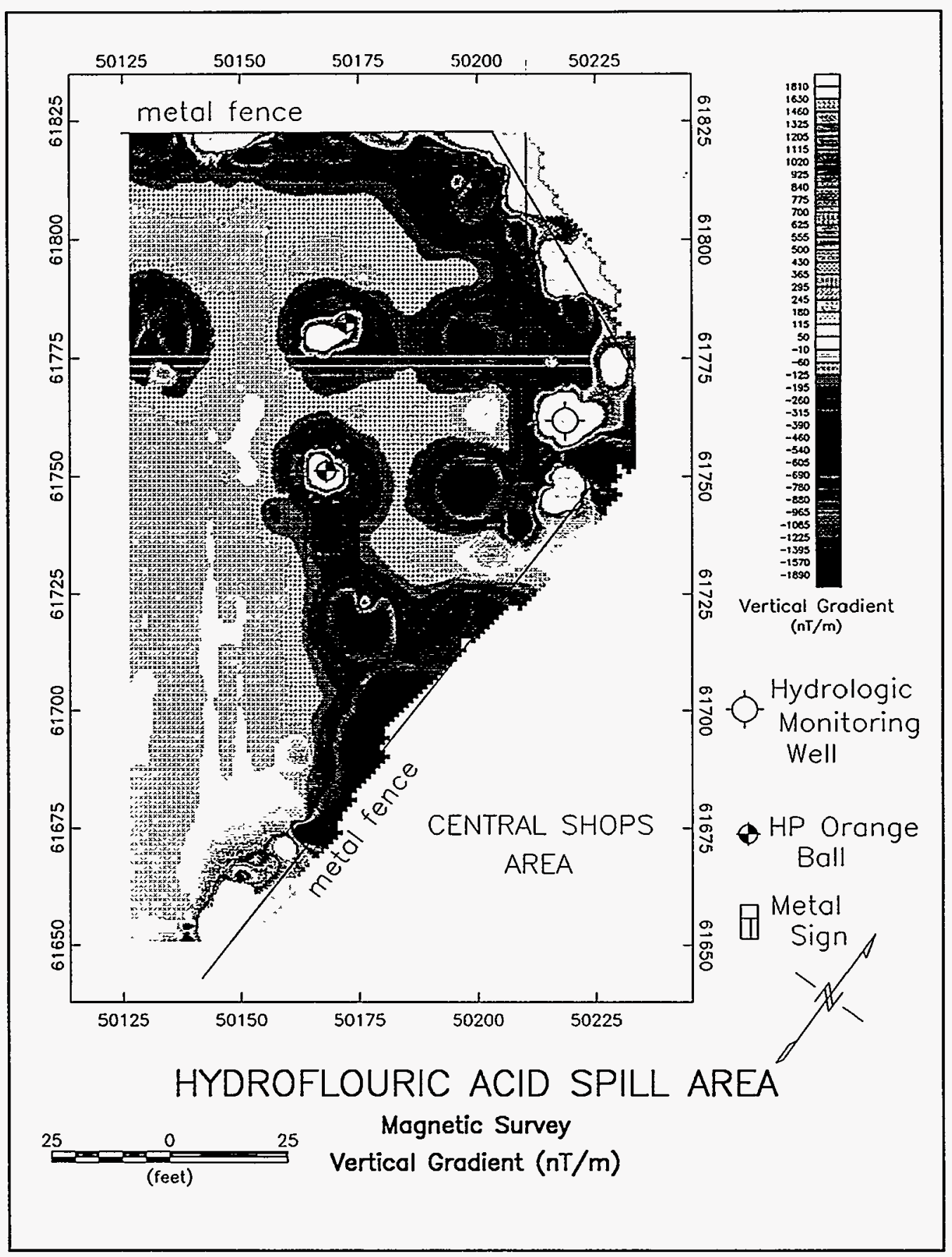




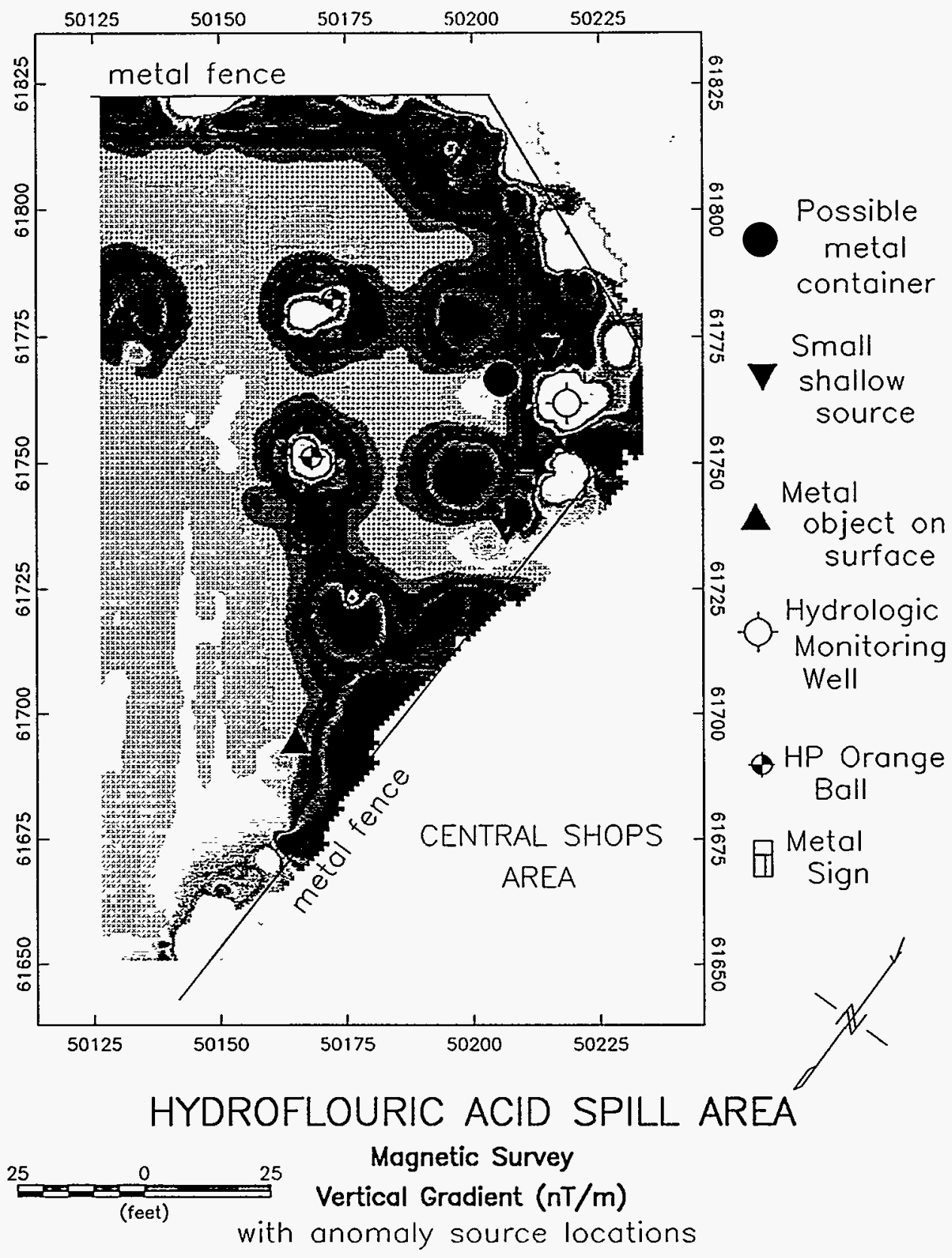

